\title{
III. «Permanente Intervention» in der Praxis: Iran als Klientelstaat der USA im Kalten Krieg, 1953-1960
}

\author{
III. 1. Intervention und Restauration: Die USA als dominierende Macht im \\ Klientelstaat Iran, 1953-1957
}

Der entscheidend durch anglo-amerikanisches Eingreifen ermöglichte Machtwechsel in Iran von Mosaddeg zu Zāhedi erschien anfangs nicht als ein historischer Wendepunkt. Eine Einschätzung von NEA im State Department warnte unmittelbar nach dem Staatsstreich vor allzu großen Erwartungen. In Iran sei weiterhin eine „Revolution“ im Gange, die Teil der allgemein zu beobachtenden „Welle des Nationalismus“ sei, die die neuen Staaten in Asien ergriffen habe. Somit sei keine grundsätzliche Kehrtwende von der Regierung des Ex-Generals zu erwarten: „The old pattern of rule has been irrevocably shattered and any leader must shape his program on the basis of nationalist aspirations. " ${ }^{\text {I }}$ Entgegen dieser Grundannahme gab es in darauffolgenden Jahren keine Neuauflage der konfliktreichen Machtteilung zwischen Hof und Mağles und auch keine Wiederkehr des politischen Wettbewerbs zwischen Schah und Ministerpräsidenten. Stattdessen vollzog sich nun eine Machtkonzentration in der Position des Monarchen und im Hof, eine Verwandlung der Funktion des Regierungschefs in die eines Befehlsempfängers Mohammad Rezās und eine weitreichende Gleichschaltung der Legislative. Zugleich stellten die Jahre vom 28. Mordād bis zu den frühen 1960er Jahren die Zeit maximalen amerikanischen Einflusses auf die inneren Gegebenheiten Irans und die Behörden und Planungsstellen des Landes dar. Wenngleich dieser Einfluss keinesfalls als amerikanische Omnipotenz gewertet werden darf, so ähnelte das bilaterale Verhältnis zwischen Washington und Teheran in eben dieser Zeit am ehesten der idealtypischen Vorstellung einer Beziehung zwischen Patron und Klientelstaat.

Der enorme Machtzuwachs gerade von Mohammad Rezā Schah war, zumindest in diesem Ausmaß, nicht erwartet worden. Wenngleich begünstigt durch seine starke konstitutionelle Rolle und die nicht zu bestreitende und weiterhin prägnante Unterstützung weiter Bevölkerungskreise für die monarchische Idee $^{2}$, schien jedoch das Verhalten des Schahs in den Krisenzeiten im Allgemeinen und während des Umsturzes im Besonderen jene Stimmen zu bestätigen, die ihm schon immer jede Befähigung als Herrscher abgesprochen hatten. Die in den vorhandenen amerikanischen und britischen Dokumenten $\mathrm{zu}$ findenden Charakterbeschreibungen Mohammad Rezās sind in der Tat wenig schmeichelhaft. Er sei „by nature a creature of indecision, beset by formless doubts and fears“, geprägt von einer „mood of stubborn irresolution“, empfänglich für schlechte Ratgeber, zudem ,lacking in courage and

\footnotetext{
${ }^{1}$ Byroade Memo, 21.8.53, FRUS 52-54, X, S. 760f.

${ }^{2}$ Vgl. dir britische Sichtweise in British Memo N ${ }^{\circ} 362,2.9 .53$, ebd., S. 780-8.

(C) Der/die Autor(en) 2021

R. Popp, Vor der Revolution, Globale Gesellschaft und internationale

Beziehungen, https://doi.org/10.1007/978-3-658-33888-6_3
} 
resolution“. 3 Nur massiver Druck seitens der Coup-Planer und letztlich die Drohung, den Staatsstreich ohne jede Beteiligung von seiner Seite zu unternehmen - implizit eine Anspielung auf ein mögliches Ende der Pahlawi-Dynastie -, konnten den Schah dazu bewegen, die jeweiligen Schriftstücke zur Entlassung Mosaddeġs und Ernennung Zāhedis zu unterzeichnen. ${ }^{4}$ Die Coup-Planer sorgten sogar für die Abwesenheit des Monarchen aus der Hauptstadt während der Ausführung der Operation, da sie an seiner Standfestigkeit zweifelten. ${ }^{5}$ Besonders gravierend war die unerwartete Flucht Mohammad Rezās nach Bagdad und Rom, die im Falle eines negativen Ausgangs des Staatsstreichs sicherlich als einer der Hauptgründe für das Scheitern bewertet worden wäre. ${ }^{6}$ Andererseits berichteten sowohl Loy Henderson wie auch Kim Roosevelt im Anschluss an den erfolgreichen Coup von einem geläuterten Mohammad Rezā, der ein ,new man“ sei, und plötzlich Entschlusskraft und Elan versprühe. ${ }^{7}$

Unmittelbar nach dem Staatsstreich stand zuerst einmal die Stabilisierung der innenpolitischen Lage im Vordergrund. ${ }^{8}$ Ausnahmezustand, Ausgangssperre in Teheran und die Schließung der Grenzen waren begleitende Maßnahmen zur Niederwerfung der letzten Widerstandsnester gegen den Coup. Sämtliche Führungsfiguren und Sympathisanten der Nationalen Front wurden verhaftet, die gegen den Staatsstreich gerichteten Demonstrationen mit Gewalt unterbunden, und die wichtigsten publizistischen Organe der Opposition eingestellt. Mit großer Entschlossenheit wurde zugleich gegen die Tudeh vorgegangen. Insgesamt wurden bis zum Jahresende über 2.000 Personen verhaftet. Tausende Sympathisanten von Nationaler Front und Tudeh wurden aus dem Staatsdienst entlassen, die Streitkräfte parallel ,gesäubert“.9 Mosaddeg selbst wurde in einem Schauprozess im November $1953 \mathrm{zu}$ einer dreijährigen Haftstrafe

\footnotetext{
${ }^{3}$ Zitate aus CIA [Donald N. Wilber], Clandestine Service History, S. vii, 30, 35, 47; ebd., Appendix B, S. 3; Tehran \#5047, 27.6.52, FRUS 52-54, X., S. 404ff., hier 406; expliziter war die Einschätzung der Rašidijān-Brüder: „Poor bastard he's scared as a dog"; zit aus FALLE, My Lucky Life, S. 82.

${ }^{4}$ Zur Drohung mit dem Ende der Dynastie vgl. ABRAHAMIAN, The Coup, S. 180f;; bereits der erste Operationsplan von CIA und MI6 enthielt die Warnung an Mohammad Rezā im Falle seiner Weigerung: „If not, Shah's dynasty will fall and US-UK backing of you will cease.“; CIA [Donald N. Wilber], Clandestine Service History, Appendix A, S. 3; ebd., Appendix B, S. 6f.; offenbar kam der Vorschlag einer solchen Drohung ursprünglich von Henderson. Vgl. CIA History Staff, The Battle for Iran, S. 39; im selben Dokument ist auch die Rede von ,extreme pressure“", infolgedessen der Schah schließlich unterzeichnete. Vgl. ebd., S. 53; anzumerken ist, dass es keinen Beweis gibt, dass Mohammad Rezā die beiden Dekrete wirklich selbst unterzeichnet hatte. Wilbers Geschichte (S. 36ff.) erwähnt am Rande, dass der Schah letztlich erst auf Drängen seiner Gattin Sorajā (S. 38: , , [...] here was an ally from a totally unexpected quarter.") signiert habe. Der CIA-Plan sah die Umsetzung in jedem Fall vor - notfalls mit ,,involuntary involvement of the Shah“. Siehe Appendix B, S. 3; ähnlich Henderson in Tehran \#481, 31.7.52, FRUS 52-54, X, S. $427 \mathrm{f}$.

5 ,Sooner or later he will begin to brood and to doubt, and at this time he must be removed from the capital to make some kind of tour.“; CIA [Donald N. Wilber], Clandestine Service History, Appendix B, S. 8; vgl. ebd., S. ix.

${ }_{6}^{6}$ „His hasty flight to Baghdad and Rome was either forgiven or forgotten in the triumph of the moment [...]“; CIA History Staff, The Battle for Iran, S. B-8; Roosevelt informierte offenbar später das CIA-Hauptquartier, dass er von der Flucht des Schahs erst aus dem Radio erfahren habe: „He just took off. He never communicated with us at all- -just took off.“; ebd., S. 59; der Schah gab nach seiner Ankunft in Bagdad vor, die Flucht sei mit Roosevelt abgesprochen gewesen. Vgl. Baghdad \#92, 17.8.53, in: NSArch EBBook No. 477, doc. 6; Roosevelt übergeht die Hintergründe in Countercoup. Wilber versuchte eine Ehrenrettung und nannte die Flucht einen ,act of prudence [...] at least partially foreseen in the plan.";CIA [Donald N. Wilber], Clandestine Service History, S. x.

${ }_{7}^{7}$ Vgl. Tehran \#466, 23.8.53, FRUS 52-54, X, S. 762-5; K. Roosevelt Memo, o.D., encl. to W. B. Smith Memo to Eisenhower, o.D. [August/September 1953], ,Iran, 1953 through 1959 (8)“, AWF, International Ser., Box 32, DDEL.

${ }^{8}$ Die CIA zeigte sich zuversichtlich, die neue Regierung könne ihre Macht ohne allzu große Probleme bewahren. Vgl. CIA, SE-49, 26.8.53, CREST: \#RDP79*001-5.

${ }^{9} \mathrm{Zu}$ den Maßnahmen vgl. GASIOROWSKI, Client State, S. 85-9.
} 
verurteilt, nach deren Verbüßung er bis zu seinem Tod 1967 in Hausarrest leben sollte. ${ }^{10}$ Der mit scharfer Rhetorik gegen Mohammad Rezā hervorgetretene ehemalige Außenminister Hosejn Fātemi wurde auf Betreiben des rachsüchtigen Herrschers zum Tode verurteilt und hingerichtet. ${ }^{11}$ Die Stammeskonföderation der Ġašğāe i im Süden Irans, die mit der MosaddegRegierung sympathisiert und diverse Avancen der CIA zur Zusammenarbeit mit den Putschisten abgelehnt hatte, entschloss sich im September 1954 zu einer Revolte nahe Schiras, gab aber kurz danach den Widerstand angesichts der geringen Chancen auf Erfolg auf. Offenbar hatte ein Besuch des CIA Station Chief Joseph Goodwin dabei eine wichtige Rolle gespielt - kolportierte Drohungen seitens Goodwins, die Stämme zu „vernichten“, sollten sie den Aufstand fortsetzen, bedeuteten zugleich das Ende der vormals engen geheimdienstlichen Beziehungen im Kontext der Stay Behind-Planungen. Die Ġašğā'i-Khane wurden im Anschluss ins Exil gezwungen. ${ }^{12}$

Infolge der Verhaftungswelle lösten sich die verbliebenen Strukturen der Nationalen Front rasch auf. Einige jüngere Kader aber formierten sich etwa einen Monat nach dem Staatsstreich in einem losen Verbund von Oppositionellen, mehrheitlich aus dem Basar-Umfeld, der „Nationalen Widerstandsbewegung Irans“ (NWI) (Nehzat-e Mog̀āwemmat-e melli-je İrān). Die NWI setzte die nationalistische Propaganda der Front fort, organisierte im Herbst und Winter 1953 einen Streik des Basars sowie eine Großdemonstration, was letztlich zu scharfen Gegenmaßnahmen der Sicherheitsorgane führte. Die NWI verzehrte sich alsbald in internen Flügelkämpfen und führte bis zum Ende der Dekade ein Schattendasein, bevor sie 1960 zum Nukleus der Zweiten Nationalen Front werden sollte. ${ }^{13}$

Die Schwäche der organisierten nationalistischen Opposition und die erfolgreiche Unterdrückung der Tudeh nach dem Coup wurde von amerikanischer Seite mit Erleichterung aufgenommen. Die CIA rechnete somit auch mit einer raschen Konsolidierung der Regierung von Fazlollāh Zāhedi, zumal Mosaddeg an Popularität eingebüßt habe: „Although many Iranians will regret the downfall of Mossadeq and will thus provide a source of future opposition to the new regime, most of this group will probably acquiesce at least temporarily

\footnotetext{
${ }^{10}$ Ausführlich zum Prozess KATOUZIAN, Struggle for Power, S. 194-207; offenbar drängte gerade Zāhedi auf eine rasche Verurteilung Mosaddeġs und dessen Hinrichtung. Der Schah hatte andererseits für den Fall einer TudehOperation zur Befreiung Mosaddeġs angeordnet, dieser solle in diesem Fall umgehend von seinen Bewachern ermordet werden. Vgl. K. Roosevelt Memos, 24.9./2.10.53, NSArch EBBook No. 435, docs. 16, 17.

${ }^{11}$ Vgl. Abrahamian, The Coup, S. 212.

${ }^{12}$ Vgl. KatouZian, Struggle for Power, S. 209f.; Gasiorowski, Client State, S. 86f., ebd., $n 3$; obskur bleibt die Zielrichtung des in der internen CIA-Geschichte des Coups erwähnten „Ġašgāa i-Amini“-Alternativplans. Vgl. CIA [Donald N. Wilber], Clandestine Service History, S.12f.; am plausibelsten ist die Interpretation, dass es sich dabei nicht um einen alternativen Umsturzplan handelte, sondern um eine Initiative mit dem Ziel, mögliche Aktionen des Stammes zugunsten Mosaddegs zu neutralisieren. Vgl. KATOUZIAN, Mosaddeq's Government, S. 15ff.; GASIOROWSKI, Coup Against Mosaddeq, S. 245; 333 n21, 335f. n39; offenbar hatte der Stammesrat unmittelbar nach dem Coup noch knapp gegen einen Aufstand gestimmt. Vgl. CIA History Staff, The Battle for Iran, S. 74; CIA, NSC Briefing, 27.8.53, CREST: \#RDP80*007-1; zur späteren oppositionellen Rolle der Ġašgà i-Khane während der Kennedy-Periode vgl. die folgenden Kapitel.

${ }^{13}$ Zur NWI vgl. CHEHABI, Liberation Movement, S. 128-34; KATOUZIAN, Struggle for Power, S. $208 \mathrm{ff}$.
} 
in the change.“14 Der NSC erwartete von Zāhedi die Etablierung einer ,moderate nationalist government" in der Hoffnung, Teile der nationalistischen Opposition zu kooptieren. Demselben Ziel diente auch Zāhedis Ankündigung einer baldmöglichsten Rückkehr zu einer verfassungskonformen Regierungsform. Bereits unmittelbar nach dem Putsch erwartete man eine spannungsgeladene Beziehung zwischen dem General und dem Schah, der keinen seiner vorherigen Premiers ,consistently“ unterstützt habe. ${ }^{15}$

Angesichts der weitreichenden Involvierung der CIA in den Staatstreich war die anschließende konsequente Unterstützung der neuen rechtsgerichteten Regierung nur konsequent. Der neue Secretary of State John Foster Dulles sprach gar von einer „Second Chance“ in Iran für die amerikanische Politik. ${ }^{16}$ Noch euphorischer formulierte der ehemalige Director of Central Intelligence (DCI) Walter Bedell Smith, inzwischen zum Under Secretary of State ernannt: „It appears that, with some assistance from us, the present leaders of Iran may be able to capitalize on the wave of public opinion that could cause a virtual renaissance in that country. " ${ }^{17}$ Winston Churchill sprach gegenüber Kim Roosevelt gar von einer ,[...] wonderful and unexpected opportunity in Iran which might change the whole picture in the Middle East.“"18 Die CIA ließ Zāhedi als Starthilfe innerhalb von zwei Tagen nach dem Coup persönlich \$5 Millionen zukommen. ${ }^{19}$ Anfang September kündigte Präsident Eisenhower sodann zusätzliche Wirtschaftshilfe über \$45 Millionen an - insgesamt erhielt die Zāhedi-Regierung 1953 etwas über $\$ 70$ Millionen an außerordentlicher Unterstützung aus den USA. ${ }^{20}$ Die umfangreichen Hilfsmaßnahmen für das neue Regime dienten offenkundig der Stabilisierung der neuen Ordnung. Allerdings war man sich in Washington nur allzu bewusst, dass der Eindruck eines übermächtigen amerikanischen Einflusses unbedingt vermieden werden musste. Bereits vor dem Coup hatte GTI im State Department gewarnt, es dürfe auf keinen Fall nach der

\footnotetext{
${ }^{14}$ CIA, SE-49, 26.8.53, CREST: \#RDP79*001-5; britische Einschätzungen sahen noch Ende 1953 bzw. Anfang 1954 eine weitaus größere Popularität für Mosaddeg. Vgl. RAHNEMA, Overthrowing Mosaddeq, S. $661 \mathrm{f}$.

15 Ebd.; vgl. 160th NSC Meeting, 27.8.53, FRUS 52-54, X, S. 771-5; hier auch die Erwartung einer wenig kompromissbereiten Position auch der neuen Regierung in den Ölverhandlungen (,General Zahedi cannot afford to seem a British stooge."; ebd., S. 772); Zāhedi bat sogar den nationalistisch eingestellten Botschafter in Washington, Allāhjār Sāleh, vorerst auf seinem Posten zu bleiben. Vgl. NSC Briefing, 27.8.53, CREST: \#RDP80*007-1.

${ }^{16}$ Vgl. 160th NSC Meeting, vorherig. Anm., S. 773.

${ }^{17}$ W. B. Smith Memo, o.D. [August 1953], ,Iran, 1953 through 1959 (8)‘, AWF, International Ser., Box 32, DDEL.

${ }^{18}$ K. Roosevelt Memo, o.D., encl. to Smith Memo, vorherig. Anm.

${ }^{19}$ Vgl. CIA [Donald N. Wilber], Clandestine Service History, S. xiii; die geheime Unterstützung wird in den späteren apologetischen Darstellungen der Affäre missverständlich wiedergegeben. Vgl. AFKHAMI, Life and Times, S. 189f.; ZAHEDI, Memoirs, Vol. I, S. 244; Zweifel über Zāhedis Qualitäten, gerade angesichts der immer wieder unterstellten Korruptionsvorwürfe gegen ihn, waren bereits in der Coup-Planung zutage getreten. Vgl. CIA History Staff, The Battle for Iran, S. 32; offenbar hatte man vergeblich nach einer besseren Alternative Ausschau gehalten wegen der „,negative aspects of his background“; ebd., S. 35.

${ }^{20}$ Neben den \$5 Millionen der CIA wurden noch weitere \$23.4 Millionen an technischer Unterstützung im Rahmen des Point Four-Programms gewährt. Zāhedi hielt die Zusagen für unzureichend und drohte mit Rücktritt. Zu den Debatten über die Hilfsleistungen vgl. MemCon, 25.8.53; Tehran \#639, 11.9.53, FRUS 52-54, X, S. 766ff., 792ff.; ebd., S. 776 n2; zum Point-Four-Programm in Iran siehe auch ed. notes in FRUS 50, I, S. 874; FRUS 50, V, S. 279; vgl. USOM Iran Report, 21.8.54, ,Iran - Background‘, Box 9, Henderson Papers, LOC; zum Projekt in Iran vgl. William E. Warne OHI, 21.5.88, OH-HSTL, S. 82-9; WARNE, Mission for Peace; AmuZEGAR, Technical Assistance; Embry, Point Four; ShIVEly, Point Four; FARMAnfarmaian, Blood and Oil, S. 315ff.; das DOD hatte dem Planning Board bez. Iran ,prompt action to insure the permanency of the Shah-Zahedi regime“ empfohlen; Memo to Chairman Planning Board, o.D. [24.8.53], ,091 Iran', Project Decimal Files, 1953; Office of Military Assistance, OASD/ISA, Box 35, RG 330, NACP; laut GASIOROWSKI, Coup Against Mosaddeq, S. 257, entsprach die Gesamtsumme ungefähr einem Drittel der kalkulierten Verluste an Öleinnahmen seit dem Mai 1951.
} 
Etablierung einer neuen pro-westlichen Führung der Eindruck entstehen, dass eine solche Regierung ein „foreign tool“ der USA sei: „Concealing the foreign hand to the maximum effect possible [...]", sei die angebrachte Vorgehensweise. Eine Beteiligung der USA am Machtwechsel gelte es im Grundsatz zu dementieren. ${ }^{21}$ Wenige Tage nach dem Coup empörte sich US-Botschafter Henderson über die um sich greifenden Gerüchte einer amerikanischen Beteiligung, angesichts seiner eigenen bedeutsamen Rolle eine doch etwas zweifelhafte Positionierung. Den Eindruck einer US-Intervention gelte es zu zerstreuen, da es ,[...] not in US interest over the long run to be given credit for internal political developments in Iran "sei, selbst für den Fall, dass, ,those developments might be to Iran's advantage [...].“22 Nicht jeder in der Eisenhower-Administration teilte diese Einsicht, zumal Zāhedi und der Schah mehrfach ihre tiefe Dankbarkeit gegenüber den USA zum Ausdruck brachten. Kim Roosevelt erinnerte sich in seinen Memoiren, Mohammad Rezā habe ihm gegenüber festgestellt: „I owe my throne to God, my people, my army-and to you!“". ${ }^{23}$ Innerhalb des PSB gab es offenbar keine Einigkeit ,[...] as to just how vigorously we should deny the accusation of the «U.S. hand» involved in the fall of Mossadegh." Wenn er schon keine umfassende Geheimhaltung amerikanischer Involvierung erreichen konnte, so empfahl Henderson aus Teheran wenigstens eine Propagandakampagne zur Stärkung des öffentlichen Images Mohammad Rezās, bevorzugt ein wohlwollendes Porträt des jungen Monarchen in einem einflussreichen amerikanischen Magazin. $^{24}$

Neben propagandistischer Unterstützung für die neue Regierung ist das große amerikanische Engagement im Anschluss an den Staatsstreich zugunsten der Stärkung der inneren Sicherheit in Iran bemerkenswert. Neben der bereits genannten Instrumentalisierung der alten Kontakte $\mathrm{zu}$ den Stammeskonföderationen zugunsten der Zāhedi-Regierung ist insbesondere die Fortsetzung der ebenfalls oben erwähnten TPBEDAMN-Operation gegen iranische Kommunisten erwähnenswert. Die bestehenden Strukturen wurden nun viel stärker zugunsten der Konsolidierung Zāhedis eingesetzt, z.B. durch Austausch von Geheimdienstinformationen über Oppositionelle, durch einige direkte Aktionen vor Ort und durch Unterstützung für das iranische Propagandabüro unter der neuen Leitung von Esfandijār Bozorgmehr. ${ }^{25}$ Langfristig nachhaltiger war die US-Unterstützung für den Aufbau effizienterer iranischer Sicherheitsorgane. Der CIA-Mitarbeiter Oberst Stephen J. Meade wurde nach Teheran entsandt, um gemeinsam mit dem dortigen Militärgouverneur und Putschisten Tajmur Baxtijār

\footnotetext{
${ }^{21}$ Vgl. Stutesman/GTI Memo, o.D. [März 1953], NSArch EBBook No. 126, doc. 3; dieses Dokument wird auch referenziert in der internen CIA-Geschichte. Siehe CIA [Donald N. Wilber], Clandestine Service History, S. 16.

${ }^{22}$ Tehran \#436, 21.8.53, FRUS 52-54, X, 759; vgl. RAHNEMA, Overthrowing Mosaddeq, S. 663f.

${ }^{23}$ RoOSEVElT, Countercoup, S. 199; zu Lobpreisungen aus Zāhedis Umfeld vgl. \#436, vorherig. Anm.; gegenüber Henderson verwandte der Schah eine ähnliche Formulierung, was Roosevelts Schilderung glaubwürdig macht. Vgl. Tehran \#466, 23.8.53, FRUS 52-54, X, S. 762-5, hier 762.

${ }^{24}$ Hier zeigte sich bereits die Präferenz Hendersons für den Schah als ,chosen instrument“ der US-Politik in Iran. Zugleich sollte der Mythos von Mosaddeg als unverzichtbarer ,grand old man“ Irans bekämpft werden. Maclean Memo, 24.9.53; Memo for the Record, 10.9.53, ,PSB 091. Iran“, WHO: NSCS, PSB Central Files Ser., Box 13, DDEL.

${ }^{25}$ Vgl. GASIOROWSKI, TPBEDAMN Operation, S. 20ff; hier auch Hinweise auf CIA-Beteiligung an den Wahlmanipulationen Zāhedis.
} 
die Grundlage für eine moderne Geheimdienstorganisation zu legen. Hier liegen die historischen Ursprünge des später notorischen SAWAK (Sāzemān-e Ettelā 'àt wa-Amnijat-e Kešwar - Organisation für Informationen / Geheimdienst und Sicherheit des Landes), formell 1956 gegründet. ${ }^{26}$

Baxtijār sollte später noch eine gewichtige Rolle in den beiderseitigen Beziehungen spielen, zuerst als erster Direktor des neu gegründeten SAWAK, in den 1960ern dann als Dissident und wichtiger Gegner des Schahs bis zu seiner Ermordung im irakischen Exil. ${ }^{27}$ Tatsächlich spielten sowohl die iranischen wie auch die amerikanischen Veteranen des Staatsstreichs im Anschluss eine gewichtige Rolle. Die beteiligten Offiziere wie Ne'mat-ollāh Nassiri oder 'Abbās Farzānegān wurden befördert und es wurden ihnen wichtige Kommandos übertragen. Der Sohn des neuen Ministerpräsidenten, Ardešir Zāhedi, wurde später iranischer Botschafter in Washington und Außenminister sowie (zeitweilig) Schwiegersohn des Schahs. Die geheimdienstlichen Protagonisten des Coups auf US-Seite wie z.B. Kim Roosevelt und George Carroll hatten im Anschluss erfolgreiche Karrieren - Roosevelt nutzte seine iranischen Kontakte später unverblümt als Consultant für diverse amerikanische Unternehmen. Die amerikanischen Coupteilnehmer bildeten den Nukleus des später bedeutsam werdenden Netzwerks von Parteigängern der Pahlawi-Monarchie in den USA, den sogenannten „Pahlavists“. ${ }^{28}$

Jenseits der unmittelbaren Unterstützungsmaßnahmen für die restaurierte Monarchie in Iran galt es nun, Weichenstellungen für die Zukunft zu treffen. Im Herbst und Winter 1953 diskutierte die Eisenhower-Administration intern ein neues Policy Statement zu Iran, das die nunmehr überholte NSC 136/1-Studie vom November 1952 ersetzen sollte. NSC 5402, verabschiedet im Januar 1954, formulierte den neuen amerikanischen Ansatz. Im Gegensatz zur Vorgängerstudie tauchte nun die indirekte Zielsetzung, Iran mittelfristig ,into active cooperation with the free world" zu bringen, wieder auf. ${ }^{29}$ Voraussetzung für eine Umsetzung sei aber die fortgesetzte Stabilisierung des Landes, insbesondere in wirtschaftlicher und finanzieller Hinsicht, was wiederum eine zeitnahe Lösung des Ölkonflikts mit Großbritannien unumgänglich mache. Bis zum Abschluss eines Kompromisses waren die USA bereit, weiterhin begrenzte finanzielle Hilfen zu leisten, wenngleich in einer Größenordnung, die den iranischen Willen zum Ausgleich mit der AIOC bewahren sollte. Die Studie sah eine Lösung vor dem April 1954, später geändert auf Juni, als wünschenswert an und deutete im Falle eines absehbaren Scheiterns der Verhandlungen zu diesem Zeitpunkt zumindest die Option einer

\footnotetext{
${ }^{26}$ Vgl. ebd.; zu Meade vgl. WiLfORD, America's Great Game, S. 99f.; Meade blieb offenbar nur bis Anfang 1955 in Iran. Baxtijār war einer der Verschwörer des 28. Mordād gewesen und hatte die von ihm geführte Division aus Kermānšāh nach Teheran geführt, griff aber nicht mehr ein. Vgl. CIA [Donald N. Wilber], Clandestine Service History, S. 73.; zu Baxtijār vgl. MiLANI, Eminent Persians: I, S. 430-7.

${ }^{27}$ Vgl. CIA History Staff, The Battle for Iran, S. 69; zu Baxtijārs Ermordung 1970 durch SAWAK-Agenten siehe unten Kapitel 7.

${ }_{28} \mathrm{Zu}$ Farzānegāns Karriere vgl. CIA History Staff, The Battle for Iran, S. 76; diese und weitere Beispiele in GASIOROWSKI, Coup Against Mosaddeq, S. 258; zu den Pahlavists grundlegend BILL, Eagle and Lion, S. 319-78. ${ }^{29}$ NSC, NSC 5402: United States Policy Toward Iran, 2.1.54, FRUS 52-54, X, S. 865-71, hier S. 866.
} 
bilateralen Lösung zwischen Teheran und Washington an, ein Fingerzeig auf ein gegebenenfalls strategisch zu begründendes Fallenlassen der finanziellen Interessen Großbritanniens. ${ }^{30}$

Politisch orientierte sich die Eisenhower-Administration gemäß NSC 5402 an der Institution der Monarchie sowie der Armee als eigentlicher Macht hinter dem Thron. Großzügige Militärhilfe sollte diese engen Beziehungen noch weiter festigen und damit die Westorientierung Irans zementieren. Begrenzte rechtliche und administrative Reformen, aber keine politischen, wurden angemahnt, zugleich aber auf die vielen bestehenden Reformhemmnisse in Iran verwiesen. ${ }^{31}$ Eine begleitende NSC Staff Study bestätigte diesen vermeintlich risikoaversen Kurs. Ausgehend von wirtschaftlicher und finanzieller Stabilisierung Irans durch US-Wirtschafts- und Finanzhilfen sowie einer zeitnahen Lösung der Ölfrage empfahl die Studie eine Konzentration auf die Stärkung der iranischen Streitkräfte mit dem Ziel, den antikommunistischen und pro-westlichen Kurs der bestehenden Regierung zu festigen, was ein Fortdauern der politischen Dominanz des Hofes implizierte. Mittelfristig hofften einige US-Offizielle wie z.B. Botschafter Henderson auf eine Integration Irans in ein nahöstliches Verteidigungsbündnis nach dem Muster der NATO und Irans, ,getting of the fence“ im Kalten Krieg, also eine eindeutige Aufgabe der Neutralität zugunsten des westlichen Blockes und somit eine Abkehr vom tradierten Kurs iranischer Außenpolitik. ${ }^{32}$

Tatsächlich enthielt NSC 5402 keine grundsätzliche Definition oder Zielsetzung der langfristigen Orientierung der US-amerikanischen Politik gegenüber Iran, abgesehen von der Stabilisierung einer antikommunistischen und pro-westlichen Führung. Wenig überraschend angesichts der Bedeutung der Loyalität der Streitkräfte während des Staatsstreiches war eine verstärkte Konzentration der US-Politik auf das militärische Feld. Allerdings gab es zwischen der Botschaft, aber auch innerhalb Washingtons, Uneinigkeit über eine realistische zukünftige

\footnotetext{
${ }^{30}$ NSC 5402, vorherig. Anm., S. 869; zur Fristsetzung siehe den Kommentar der FRUS-Editoren in ebd. $n 2$; bezüglich einer bilateralen Option hatten sich DOD und JCS gegen starken Widerstand des State Departments größtenteils durchgesetzt und den April festgesetzt. Siehe 178th NSC Meeting, 30.12.53, FRUS 52-54, X, S. 859 n4; die JCS hatten auf den April 1954 als Deadline gedrängt. Vgl. JCS Memo, 29.12.53, ,Iran (4)‘, WHO, NSCS, Disaster File Ser., Box 65, DDEL; hier auch eine Version des Paragraphen 27, der in der FRUS-Version fehlt, und das Vorgehen im Falle eines sowjetischen Angriffs definiert. Der Juli wird noch als Frist genannt in Executive Secretary Note to NSC, NSC 175, 21.12.53, ,NSC 175', Box 26, S. 8; NSC 175 war eine Vorgängerversion der letztlich verabschiedeten Studie. Die Verwirrung der FRUS-Editoren ist eine Folge der erneuten Verschiebung der Frist auf den Juni nach einer OCB-Anfrage vom April 1954. Vgl. OCB Progress Report on NSC 5402, 15.4.54, ebd., ,NSC 5402‘, Policy Papers, Box 27; Lay Memo, 30.4.54, ,194th Meeting‘, Official Meeting Minutes, Box 10, RG 273, NACP.

${ }^{31}$ Vgl. NSC 5402; im PSB sah man den Schah kritisch: ,There is no hope that we can develop an adequate leader out of the present Shah, but as long as he remains in power we must probably support him.“; gleichzeitig war man sich bewusst, dass ,[...] g[G]etting rid of the old conservative landowning class which now controls the Government is going to be a long process."; der PSB empfahl „steady pressure“ für mehrere Jahre „, [...] to improve the character of the Parliament and of the Government officials."; Debevoise Memo, 22.10.53, ,PSB 091.Iran", WHO, NSCS, PSB Central File Ser., Box 13, DDEL; HEIKAL, Return of the Ayatollah, S. 67, erwähnt ein ihm nach der Revolution angeblich gezeigtes Plandokument, erstellt seitens der CIA, das ein umfangreiches Reformund Propagandaprogramm vorsah, und im Kern bereits die spätere Weiße Revolution vorweggenommen habe. Keines der freigegebenen bzw. in den Archiven verwahrten Dokumente enthält einen solchen Plan. Die tatsächliche Politikfestlegung durch NSC 5402 widerspricht derlei ambitionierten Zielsetzungen zu diesem Zeitpunkt.

32 NSC Staff Study, o.D. [Januar 1954], FRUS 52-54, X, S. 886; weiterhin klassifizierte Teile der NSC Staff Study zu NSC 5402 lassen sich von einer vollständigen Version der Staff Study zur Vorgängerstudie NSC 175 rekonstruieren. Vgl. NSC Staff Study, 21.12.53, ,Annex to NSC 175`, Policy Papers, Box 26, RG 273, NACP.
} 
Aufgabenstellung für die iranischen Streitkräfte, insbesondere was Verteidigungsmaßnahmen entlang der Grenze zur UdSSR anging. NSC 5402 erwähnte die bislang gelieferte Militärhilfe in Höhe von \$46 Millionen und bereits vereinbarte aber noch ausstehende zusätzliche Lieferungen in Höhe von \$58 Millionen, verwies aber auf den vergleichsweise niedrigen Ausbildungsstand und die mangelnde Professionalität iranischer Truppen, die die Absorption selbst dieses relativ geringen Volumens an Rüstungsmaterial erschweren würden. Gegenwärtig seien die Streitkräfte zweifelsfrei in der Lage die innere Sicherheit in Iran zu gewährleisten mit der Ausnahme eines - reichlich unwahrscheinlichen - Aufstands sämtlicher Stämme zum gleichen Zeitpunkt. Bei entsprechender US-Unterstützung bezüglich Training und Ausrüstung seien begrenzte Guerilla- und Verzögerungsoperationen im Falle eines sowjetischen Einmarsches in Iran vorstellbar, aber ,[...] it is unlikely that they could in themselves become capable within the foreseeable future of effectively delaying a strong Soviet thrust toward Iraq or the Persian Gulf.“33 In einem partiellen Entgegenkommen des DOD gegenüber den Forderungen Hendersons aus Teheran stellte NSC 5402 zumindest ein „long-range program of improving the Iranian armed forces“ in Aussicht, wenngleich unter der Voraussetzung einer Umsetzung der Verteidigungspläne auf regionaler Basis und Zusagen militärischer Unterstützung der Nachbarstaaten für Iran. ${ }^{34} \mathrm{Zu}$ diesem Zeitpunkt überwogen aber noch die Bedenken über die möglichen Folgen einer ostentativen Westbindung Irans, zumal sich die Sowjetunion bereits während der Aserbaidschankrise auf die weiterhin bestehende Selbstverpflichtung des südlichen Nachbarn zu Neutralität, basierend auf den sowjetischiranischen Verträgen von 1921 und 1927, berufen hatte. Moskau interpretierte die Verträge weiterhin als Ermächtigung zur militärischen Intervention im Falle einer Bedrohung der Sowjetunion von iranischem Territorium aus. ${ }^{35}$

Jenseits der Fragen der zukünftigen politischen Ordnung in Iran bzw. des Ausmaßes und der Zielsetzung eines erweiterten amerikanischen Militärhilfeprogramms stand ein baldmögliche vertragliche Lösung des Ölkonflikts im Zentrum von NSC 5402. Die Einnahmen aus der

\footnotetext{
${ }^{33}$ NSC 5402, 2.1.54, FRUS 52-54, X, S. 868; trotz des „Erfolgs“ des Staatsstreichs vom August 1953 hatte man innerhalb der CIA einen schlechten Eindruck von der Qualität der Kader in den iranischen Streitkräften gewonnen. Der ,typische“ iranische Offizier sei geprägt von einer „,modern tradition of defeat“ und würde, ähnlich wie der Schah, Unentschlossenheit und Inferioritätsempfinden durch Schwulst und Großspurigkeit kompensieren. Vgl. CIA [Donald N. Wilber], Clandestine Service History, Appendix E, S. 2 f.

${ }^{34}$ NSC 5402, vorherig. Anm., S. 868; die Leitlinien der Studie sahen Militärhilfe in einem Ausmaß vor, die Fähigkeiten der Streitkräfte zu erweitern, ,[... to maintain internal security and provide some resistance to external aggression", wobei der zweite Satzteil erst durch Beschluss des NSC eingefügt wurde. Vgl. ebd., S. $831 n 2$; der NSC nahm eine Eingabe der JCS auf, die de facto den Einsatz von US-Truppen zur Verteidigung Irans ausschloss. Siehe 178th NSC Meeting, 30.12.53, FRUS 52-54, X, S. 863 n8; die Skepsis der JCS bezüglich der Umsetzbarkeit einer Verteidigung Irans und ihre Zweifel an der Realisierbarkeit einer regionalen Verteidigungsorganisation zu diesem Zeitpunkt widerlegen im Grunde sowohl die Gavin- wie die Brands-Thesen bez. der US-Entscheidung zugunsten TPAJAX im Vorjahr. Vgl. Gavin, Politics, Power, and U.S. Policy; Brands, Cairo-Teheran Connection, S. 452-6; ähnlich PERSSON, Baghdad Pact, S. 157f.

${ }^{35}$ Die „Legal Experts“ im State Department bestritten diese Interpretation als unvereinbar mit der UN-Charter, konzedierten aber zugleich, der Vertrag von $1921,[\ldots]$ does provide the Soviets with a plausible-sounding pretext for introducing troops"; Part 6 in NSC Staff Study, 21.12.53, ,Annex to NSC 175', Policy Papers, Box 26, RG 273, NACP; vgl. CIA, NIE 30-55, 21.6.55, FRUS 55-57, XII, S. 92; MemCon, 13.1.56, ebd, S. 236; zu den Unterschieden zwischen den iranisch-sowjetischen Verträgen von 1921 und 1927 vgl. OCB Draft Report (NSC 5504), 8.6.55, ,OCB 091. Iran (File \#2) (8) ${ }^{6}$, WHO, NSCS, OCB Central File Ser., Box 43, DDEL; vgl. ATKIN, Soviet-Iranian Relations, S. $107 \mathrm{ff}$.
} 
Ölförderung waren unersetzbar sowohl für die Stabilisierung des Landes als auch für die Umsetzung jedweder Reformmaßnahmen. Die begleitende Staff Study hatte bereits die zu erwartenden Probleme skizziert. Ausgeschlossen war eine Rückkehr zum Status Quo Ante Mosaddeg - trotz dessen Scheiterns gebe es eine einhellige Überzeugung der öffentlichen Meinung in Iran, dass der Schritt der Nationalisierung richtig und notwendig gewesen war. Ein Erzwingen allzu weitreichender iranischer Konzessionen würde zweifelsfrei das Überleben der Zāhedi-Regierung gefährden. Andererseits hätten die jüngeren Entwicklungen auf dem Welterdölmarkt die iranische Verhandlungsposition entscheidend geschwächt. Es gab ein gewaltiges Überangebot an Öl und die Nachbarstaaten hatten den iranischen Produktionsausfall in der Zwischenzeit problemlos durch Mehrförderung aufgefangen. Eine Rückkehr des iranischen Öls auf den Weltmarkt war zudem nur im Rahmen einer kooperativen Lösung unter Einschluss der sieben großen multinationalen Ölunternehmen, den Seven Sisters, zu denen auch die AIOC zählte, möglich, da diese allein aufgrund ihrer Kontrolle der Vertriebswege in der Lage waren, eine entsprechende Absatzquote bei anhaltender Preisstabilität zu garantieren. ${ }^{36}$ Von britischer Seite waren die Befindlichkeiten die gleichen wie vor Mosaddeġ Sturz, zuvorderst die Wahrung des eigenen Prestiges und eine angemessene Entschädigung für die Nationalisierung der britischen Interessen in Iran. Für die USA ging es in erster Linie um eine rasche Lösung auf Basis eines für beide Seiten akzeptablen Kompromisses, der sowohl die wirtschaftliche Grundlage für die Stabilisierung Irans schaffen, zugleich aber die britische Position im Nahen und Mittleren Osten bewahren würde. Zuletzt wirkte auf amerikanischer Seite auch ein wirtschaftliches Eigeninteresse insofern, als Sorge getragen werden sollte, dass eine Vereinbarung ,[...] must not establish a precedent adversely affecting the presently established international oil industry in a way inimical to U.S. interests. “37

Die britische Seite hatte bereits vor dem Staatsstreich auf amerikanisches Drängen hin größere Kompromissbereitschaft gegenüber einer Nachfolgeregierung zugesagt. Churchill selbst gestand gegenüber Kim Roosevelt ein, die AIOC „,fouled things up“ in der Vergangenheit und versprach Besserung. ${ }^{38}$ Herbert Hoover, Jr., Sohn des ehemaligen US-Präsidenten, mit langjähriger Erfahrung im Ölgeschäft, wurde im Oktober 1953 als Sonderemissär mit der Führung der schwierigen Verhandlungen beauftragt. Die kooperative Lösung der Ölfrage in Iran unter Beteiligung aller großen Ölkonzerne sollte mittels Gründung eines internationalen Konsortiums bewerkstelligt werden. Tatsächlich hatte man bereits Mosaddeg zum Jahresbeginn 1953 eine ähnliche Lösung unterbreitet und die Konsortiumslösung wurde intern im Anschluss auch weiterhin als der erfolgversprechendste Ansatz angesehen. ${ }^{39}$ Die

\footnotetext{
${ }^{36}$ Vgl. NSC Staff Study, o.D. [Januar 1954], FRUS 52-54, X, S. 871-8.

${ }^{37}$ Ebd., S. 877.

${ }^{38}$ CIA [Donald N. Wilber], Clandestine Service History, S. 82; die offizielle britische Zusage während der TPAJAX-Planungen war allerdings reichlich evasiv formuliert. Sie ist abgedruckt in ebd., Appendix C; siehe auch CIA History Staff, The Battle for Iran, S. 39f.

${ }^{39}$ Vgl. Mosaddegs vorläufige Zustimmung zu einer solchen Lösung in Tehran \#2506, 2.1.53, FRUS 52-54, X, S. 575-7; die spätere Anteilsverteilung im Konsortium bereits vorgezeichnet in State \#4403, 3.1.53, ebd., S. 580f.; nach dem Coup widergespiegelt in State \#853, 23.9.53, ebd., S. 802ff.; Fraser Letter, 3.12.53, o.F, Lot 57 D 155, Subject Files 1947-54, NEA/GTI, Box 41; FO to Gifford, 14.1.53, FOCP V, B, 5, S. 125-8; Details zu einer
} 
amerikanischen Unterhändler konzentrierten sich von Anbeginn der langwierigen Verhandlungen auf eine kooperative Lösung unter Einschluss der Majors, dabei zwischen taktischen Parteinahmen für die iranische und britische Seite changierend. Mit Geschick und sanftem Druck überzeugten die US-Offiziellen Zāhedi von der Notwendigkeit, die diplomatischen Beziehungen zu London wieder aufzunehmen, ein Schritt, der bereits im Dezember erfolgte und die Erfolgsaussichten der Verhandlungen erheblich verbesserte. ${ }^{40} \mathrm{Im}$ Anschluss verstärkten die Amerikaner sodann den Druck auf die britische Führung und auf die AIOC und überzeugten beide, von ihren Maximalforderungen abzurücken. Die Briten mussten ihre Forderungen nach einem ,controlling share“ für die AIOC, einer Mehrheit der Anteile des zu formierenden Konsortiums, aufgeben, da eine Rückkehr zu einer britischen Dominanz in der iranischen Ölwirtschaft politisch nicht durchzusetzen war. ${ }^{41}$

Nach komplizierten Verhandlungen einigte man sich auf eine Begrenzung der AIOCBeteiligung am Konsortium auf 40\%. Ebenfalls 40\% gingen an einen Zusammenschluss der fünf amerikanischen Majors: Standard Oil of New Jersey, Socony-Vacuum Oil Company, Standard Oil of California, die Gulf Oil Corporation und die Texas Company. Von den verbliebenen Anteilen gingen $14 \%$ an die Royal Dutch/Shell, die selbst wiederum zu $40 \%$ in britischem Besitz war, was rechnerisch in einer britischen Gesamtbeteiligung am Konsortium von weniger als 50\% resultierte. Mit der Aufnahme der französischen Compagnie Française de Pétrole ins Konsortium für weitere 6\% schien allerdings der britische Einfluss gesichert, da diese Gesellschaft die Reputation besaß, sich gegenüber der AIOC weitgehend linientreu zu verhalten. ${ }^{42}$ Politisch noch brisanter waren die anschließenden Verhandlungen ab April 1954 über eine angemessene Entschädigung für die nun nicht mehr grundsätzlich in Frage gestellte Nationalisierung der iranischen Ölförderung. Hauptstreitpunkt war dabei die anfängliche Forderung der AIOC nach Entschädigung für entgangene Profite in der Zukunft, was sich

„,international marketing company“ bereits in Wilkins Draft Memo, 10.8.53, NSArch EBBook No. 126, doc. 4; eine verwandte Lösung über den Aufkauf der AIOC-Ansprüche durch ein Konsortium der amerikanischen Konzerne bereits in Amory Jr. Memo, 28.11.52, CREST: \#RDP79*058-0; der spätere britische Premier nannte eine solche Gesellschaft, with joint Anglo-American control“bereits im Oktober 1951 als einzige Hoffnung. Siehe den Eintrag vom 3.10.51, in: CATTERALL, Macmillan Diaries: I, S. 105.

${ }^{40}$ Vgl. Tehran \#535, 31.8.53; Tehran \#866, 9.10.53; State \#1153, 9.11.53; Tehran \#1088, 12.11.53, Tehran \#1140, 19.11.53; London \#2158, 19.11.53; Tehran \#1227, 3.12.53, FRUS 52-54, X, S. 777ff., 810-3, 828f., 829ff., 838f., 842f., 846ff.; ebd., S. 778 n3; Joint Communiqué, 5.12.53; Eden to Wright, No. 2, 17.12.53, FOCP V, B, 5, S. 236, 238ff.; für die kuriosen Umstände vgl. WRIGHT, Restoration, S. 161-5; zur Schweizer Vermittlungsrolle vgl. MEIER, Helvetiens guter Draht, S. 166ff.

${ }^{41}$ Vgl. London \#2908, 7.1.54; 180th NSC Meeting, 14.1.54; State \#Tedul 50, 15.2.54; Merchant Memo, 16.2.54; State \#1749, 19.2.54; State \#4334, 23.2.54, FRUS 52-54, X, S. 893f., 897f., 923f., 924f., 926ff., 930f.; tatsächlich sah man auch in London ,strong arguments for giving the big American oil companies a share in the future marketing of Persian oil“". Die Rückkehr Irans auf den Ölmarkt bedinge Produktionseinschränkungen in anderen Fördergebieten des Nahen Ostens, was wiederum nur mit Zustimmung der US-Konzerne zu erreichen war. Siehe C.C.(54) 1st Conclusions, 7.1.54, CAB 21/3934, TNA:PRO.

${ }^{42}$ Vgl. London \#4311, 1.4.54, FRUS 52-54, X, S. 972-5; der Gesamtwert des Konsortiums wurde auf \$1 Milliarde festgelegt - die der AIOC zustehende Zahlung von $\$ 600$ Millionen für die abgetretenen Anteile erfolgte größtenteils über eine Beteiligung an den Erlösen der anderen Gesellschaften in den Folgejahren. Zu den Verhandlungen vgl. als locus classicus HEISS, Iranian Consortium, S. 515-20; siehe auch MARTSCHUKAT, Antiimperialismus, S. 302-12; PAINTER, Oil and the American Century, S. 192-8; BAMBERG, History of BP: II, S. 490-511; GoODE, United States and Iran, S. 127-53; die US Majors hatten bereits früh zugestimmt, 5\% ihrer Gesamtbeteiligung am Konsortium an eine Gruppe amerikanischer Independents abzutreten. Die Iricon Agency, ein Zusammenschluss von neun unabhängigen Gesellschaften, trat 1955 dem Konsortium bei. Vgl. Waugh Memo, 2.5.55, FRUS 55-57, XII, S. 738 . 
bereits in den Verhandlungen mit der Mosaddeg-Regierung als kaum lösbares Problem erwiesen hatte. Massiver amerikanischer Druck erzwang hier britisches Einlenken im März 1954 - „This whole thing can affect our partnership in the Near East“, warnte Secretary of State Foster Dulles den britischen Botschafter unmissverständlich. ${ }^{43}$ Im Anschluss an diese kurze aber vehemente Krise und die Einigung zwischen AIOC und den anderen Majors gelang die rasche Lösung der noch offenen Fragen, wenngleich die gesetzte Frist bis Juni 1954 nicht mehr einzuhalten war. ${ }^{44}$ Trotz bestehendem Misstrauens kamen die anschließenden Verhandlungen zwischen dem Konsortium und der iranischen Regierung recht zügig bis August zum Abschluss, was vor allem dem geschickten Management der US-Regierung zuzuschreiben war. Die amerikanischen Offiziellen wiesen übertriebene Forderungen beider Seiten rasch in die Schranken und manövrierten London, Teheran und die Ölgesellschaften mit wohl dosiertem Druck auf den Weg zu tragfähigen Kompromissen.

Die Kernfrage der Kontrolle der Ölindustrie wurde in der finalen Vereinbarung vom 15. September mittels semantischer Angleichung an das Nationalisierungsgesetz von 1951 gelöst, zuvorderst mit Blick auf die notwendige Ratifikation durch den neuen Mağles - bei tatsächlicher effektiver Dominanz des Konsortiums über die operativen Vorgänge. Einem ähnlichen Muster folgte die Lösung der Entschädigungsfrage: Die Iraner akzeptierten den britischen Anspruch im Grundsatz, die letztlich vereinbarte Summe stellte aber nur einen Bruchteil der ursprünglichen Forderung dar. Die NIOC behielt formell das Besitzrecht über das iranische Öl, die Ausbeutung, Exploration und Verarbeitung desselben aber überließ sie den Betriebsgesellschaften des Konsortiums, mit stark begrenzter iranischer Beteiligung und Supervision. Das Konsortium sagte im Gegenzug die schrittweise Rückkehr des iranischen Erdöls auf den Weltmarkt zu, wobei über Umwege 50\% der Reinerlöse aus der Produktion an Iran entfielen, womit die etablierte 50:50-Profitteilung am Persischen Golf ebenfalls gewahrt würde. Die Konsortiumsvereinbarung hatte eine Laufzeit von 25 Jahren, mit der Option, nach Belieben der Konzerne, dreier weiterer Verlängerungen für jeweils fünf Jahre nach $1979 .{ }^{45} \mathrm{Um}$

43 „If the partnership dealings always end in dismal failure, it can't go on, and we will take a new look.“; TelCon, 17.3.54, , Telephone Memos, [...] 1954 (3)‘, JFDP, Telephone Conversations Ser., Box 2, DDEL; für die britische Wiedergabe des Gesprächs vgl. HeIss, Iranian Consortium, S. 521f.; eine Wiedergabe des Gesprächs in einem CIA-Dokument ging noch weiter: „U.S. policy would attempt to save both Iran and the British investment there, but given a choice between the two, would save Iran."; Memo for the DCI, 18.3.54, CREST: RDP80*010-0; die NSC 5402 OCB Working Group empfahl starken Druck auf London, gerade hinsichtlich einer möglichen Kürzung der US-Wirtschaftshilfen an Großbritannien. Siehe Bishop Memo, 22.3.54, , OCB 091. Iran (File \#1) (3)‘, WHO, NSCS, OCB Central File Ser., Box 42, DDEL; siehe auch London \#3992, 17.3.54; London \#4015, 18.3.54, FRUS 52-54, X, S. 950-3, $953 \mathrm{ff}$.

${ }_{44}$ Zum Kompromiss innerhalb des Konsortiums zu den Entschädigungszahlungen an die AIOC siehe London \#4150, 25.3.54, ebd., S. 968ff.; zum Verlauf der weiteren Verhandlungen vgl. 199th NSC Meeting, 27.5.54, ebd., S. 1008-12; HeISs, Iranian Consortium, S. $523 \mathrm{ff}$.

${ }_{45} \mathrm{Zu}$ der finalen Phase der Verhandlungen vgl. ebd., S. 525-32; vgl. Dulles Memo, 3.8.54, FRUS 52-54, X, S. 1043f.; auf Druck der US-Regierung akzeptierten die Majors, zukünftig als , agent" der NIOC in Iran zu operieren, wenngleich zuvor vereinbart worden war, dass diese Formulierung nicht im Wortsinn umzusetzen sei. Letztlich vermied die finale Vereinbarung die Erwähnung eines ,agent“. Vgl. ebd., S. 1026 n3; London \#5750, 15.6.54, ebd., S. 1028f.; zur Entschädigung vgl. Tehran \#307, 5.8.54, ebd., S. 1044ff.; die Iraner akzeptierten eine Entschädigungszahlung von etwa \$70 Millionen für die nationalisierten Werte in Iran und die dadurch der AIOC vor 1954 entstandenen Verluste, laut Heiss ,[...] so low a figure [...] that even Mossadegh might have accepted it." ( dies., Iranian Consortium, S. 531); zudem sagte die AIOC die Freigabe der aus dem nie ratifizierten Supplemental Agreement von 1949 ausstehenden Zahlungen über etwa \$140 Millionen zu, was in der Aufrechnung 
den amerikanischen Konzernen eine Mitwirkung am Konsortium zu ermöglichen, musste die Eisenhower-Administration im Interesse der nationalen Sicherheit den Majors Immunität im Rahmen der Anti-Trust-Bestimmungen zusagen. ${ }^{46}$

Mit der Zustimmung des Mağles und des Senats zur Konsortiumsvereinbarung im Oktober 1954 und dem Inkrafttreten der Vereinbarung war die seit 1951 währende iranische Ölkrise auch formell beendet. ${ }^{47}$ Augenfällig ist dabei der Kontrast zum vorausgegangenen Scheitern der UdSSR und Großbritanniens in den Jahren 1947 und 1949, von ihnen gesponserte Ölvereinbarungen durchs iranische Parlament zu bringen - die Konsortiumsvereinbarung unter maßgeblicher amerikanischer Beteiligung symbolisiert somit auch in diesem Aspekt den Übergang zur Dominanz der USA in Iran. Angesichts der weitreichenden Folgen und der impliziten Stabilisierung muss das Abkommen zweifelsfrei als großer amerikanischer Erfolg bewertet werden, zumal auch nach dem Sturz Mosaddegs das Erreichen dieser Einigung keineswegs selbstverständlich gewesen war. Die positiven gesamtstrategischen Konsequenzen einer Rückkehr zur (pro-westlichen) Stabilität Irans gerieten angesichts der eskalierenden Konfrontation mit der arabisch-nationalistischen Regierung in Ägypten, die schließlich in die Suezkrise von 1956 münden sollten, bald in Vergessenheit. Andere langfristige Auswirkungen der Konsortiumsgründung sind aber offenkundig. David Painter bewertet das Abkommen als Grundstein für eine fortgesetzte Kontrolle der globalen Erdölökonomie durch die etablierten Majors für weitere anderthalb Jahrzehnte. ${ }^{48}$ Der Preis für die Einigung aber war die verhandelte Abkehr vom tradierten Konzept einer Konzessionsgewährung inklusive weitreichenden Souveränitätsverzichts zugunsten einer zumindest formell gleichberechtigten Nutzungsvereinbarung bei Anerkennung des grundsätzlichen Besitzanspruches Irans auf seine Rohstoffvorkommen. ${ }^{49}$ Bezüglich der Beziehungen zwischen den USA und Großbritannien im nahöstlichen Raum ist zu konstatieren, dass die Konsortiumsvereinbarung gleichzeitig Bekräftigung und Schwächung des im Zweiten Weltkrieg entstandenen latenten angloamerikanischen Kondominiums darstellte. Die Koordination zwischen London und Washington blieb eng und die Amerikaner drängten niemals auf eine - machtpolitisch durchaus durchsetzbare - vollständige Verdrängung der britischen Position in Iran. Ohne US-

somit die Kompensation noch übertraf. Weitere schwierige Fragen waren Londons Forderung, der Hauptsitz des Konsortiums müsse in Großbritannien liegen, die Berufung eines britischen Generalmanagers und die Abwicklung sämtlicher finanzieller Transaktionen in Pfund Sterling, allesamt dominierenden britischen Einfluss implizierend. Letztlich wurden zwei Betriebsgesellschaften mit Sitz in Iran und eine Holdinggesellschaft in London gegründet. Zudem akzeptierten die Briten einen niederländischen Manager. Vgl. MARTSCHUKAT, Antiimperialismus, S. 31520.

${ }_{46}$ Vgl. ebd., S. 300f., 308ff.; vgl. auch die NSC 5402 Staff Study in FRUS 52-54, X, hier S. 888f.; sowie Cutler Memo, 4.1.54; Brownell Letter, 20.1.54; State \#532, 15.9.54, ebd., S. 864f., 901-4, 1049f.; vgl. KAUFMAN, Mideast Multinational Oil, S. $953 \mathrm{f}$.

${ }^{47}$ Der Mağles stimmte dem Abkommen am 21. Oktober mit 113:5 Stimmen zu, der Senat folgte am 28. Oktober mit 41:4 Stimmen. Mit Unterzeichnung durch den Schah am 29. Oktober trat der Vertrag mit dem Konsortium endgültig in Kraft. Vgl. Tehran \#385, 15.8.54; State \#843, 28.10.54, FRUS 52-54, X. S. 1047ff., 1062.

${ }^{48}$ Vgl. PAINTER, Oil and the American Century, S. 197f., $206 f$.

${ }^{49}$ Prägnant hier Yergin, The Prize, S. 476f.; für eine interessante These zu den mittelfristigen Effekten der iranischen Ölkrise auf die Formulierung eines Konzept der ökonomischen Souveränität postimperialer ",neuer“ Staaten und die langfristige Infragestellung der Konzessionsregime in der globalen Ölökonomie vgl. DIETRICH, Mossadegh Madness; vgl. nun auch ders., Oil Revolution, S. 26-88. 
Unterstützung wäre eine Rückkehr der AIOC, bald in British Petroleum Company umbenannt, nach Iran wohl kaum möglich gewesen. Andererseits büßte sie ihre vorherige Monopolstellung ein, wenngleich im Nachgang die von den anderen Konsortiumsteilnehmern geleisteten Finanztransfers als erstaunlich vorteilhaft erschienen. ${ }^{50}$

Für den Komplex der amerikanisch-iranischen Beziehungen ist es dennoch wichtig zu betonen, dass die USA während der Verhandlungen über die Gründung eines Konsortiums keine tatsächliche Äquidistanz zwischen den Parteien einnahmen. Trotz teilweise scharfer Auseinandersetzungen mit London begünstigte die Eisenhower-Administration letztlich eine den britischen Interessen stark entgegenkommende abschließende Regelung. ${ }^{51}$ Die nominelle Anerkennung der Nationalisierungsbeschlüsse und die Akzeptanz der nationalen Ölgesellschaft als Vertragspartner konnten nicht darüber hinwegtäuschen, dass Mosaddeġs zentrale Forderung einer iranischen Kontrolle der Öloperationen weit verfehlt wurde. Tatsächlich fixierte das Abkommen nur das Produktionsausmaß für die ersten drei Jahre - im Anschluss wurde eine geheime Absprache zwischen den beteiligten Konzernen wirksam, Aggregate Programmed Quantity genannt, infolge derer die Produktionsmenge in Iran mit Blick auf die allgemeine Angebotslage in den Folgejahren reguliert wurde. ${ }^{52}$

Der in den Quellen wahrnehmbare Schwenk der US-Politik weg von einem möglichst fairen Ausgleich zwischen den Standpunkten Irans und Großbritanniens im Herbst 1953 und hin zu der iranische Interessen weitgehend ignorierenden Vereinbarung vom Oktober 1954 ist am plausibelsten mit der amerikanischen Selbstwahrnehmung der eigenen Einflussmöglichkeiten in Iran zu erklären. Solange keine Öleinnahmen zu verzeichnen waren, war das Land in seiner Existenz abhängig von einer Fortsetzung der amerikanischen Unterstützungsleistungen. Einer Einschätzung der CIA zufolge waren die außerordentlichen Unterstützungsleistungen aus den USA im Juni 1954 aufgebraucht, was auf iranischer Seite die Bereitschaft zur Einigung, selbst

\footnotetext{
${ }^{50}$ Siehe Yergin, The Prize, S. 478; auch MARsh, Cold War Oil, S. 167, sieht British Petroleum im Anschluss in einer stark verbesserten wirtschaftlichen Position; BAMBERG, History of BP: II, S. 511, konstatiert, eine Rückkehr zum Monopol „, [...] would probably have been a commercial embarrassment“ angesichts des bestehenden Überangebots an Öl; HEISS, Iranian Consortium, S. 525, spricht dennoch von der Vereinbarung vom Oktober 1954 als Symbol dafür, dass der , ,...] mantle of Western power in Iran had already passed from London to Washington."; ähnlich ELM, Oil, Power, and Principle, S. 330f.; der Namenswechsel erfolgte noch im Dezember in Anerkennung der Tatsache, dass der Schwerpunkt der Konzernoperationen nun nicht mehr im Iran beheimatet war.

${ }^{51}$ Neben der oben erwähnten nachdrücklichen Warnung im März 1954 ist sicherlich die Vereitelung reichlich durchsichtiger britischer Versuche zu nennen, über den Umweg der Auszahlung der iranischen Erdölerlöse in Pfund Sterling und Beschränkungen der Konvertierbarkeit in US-Dollar die de facto-Kontrolle über die iranische Volkswirtschaft zu gewinnen. Hoover Jr. erwähnte Versuche Londons, ,[...] to obtain the UK, pre-audit' control of Iranian conversion of sterling into dollars, thus achieving complete British domination of Iran's economy."; MemCon, 1.6.54, ,OCB 091. Iran (File \#5) (4)‘, WHO, NSCS, OCB Central File Ser., Box 44, DDEL; darüber hinaus würde eine solche Regelung ,[...] considerably hamper dollar loans and U.S. trade with Iran."; Richards Memo, 28.5.54, ebd.; vgl. State \#853, 23.9.53, Tehran \#2382, 21 .5.54; Tehran \#10, 1.7.54; State \#44, 8.7.54, FRUS 52-54, X, S. 802ff., 1004-7, 1035ff., 1039ff.; ebd., S. 1023 n6; Makins Letter, 3.7.54, FOCP V, B, 7, S. 182ff.; vgl. HeISs, Iranian Consortium, S. 524, 526; MARTSCHUKAT, Antiimperialismus, S. 322, betont zutreffend die Halbherzigkeit dieser britischen Manöver.

${ }_{52}$ Details bei HEISS, Empire and Nationhood, S. 215; scharf aber durchaus zutreffend das Urteil bei ABRAHAMIAN, The Coup, S. 207-10; zu den internen Konsortiumsabsprachen über Fördermengen vgl. Akins Letter, 18.3.68, PET 6 IRAN, CF 67-69; die Absprache wurde offenbar erst 1974 öffentlich.
} 
zu schlechten Konditionen, steigerte. ${ }^{53}$ Ölvermittler Hoover Jr. sprach während der Verhandlungen intern von notwendigem „strong pressure“ auf die Iraner, um deren Zustimmung zu erreichen, gepaart mit der Aussicht, dass ,[...] certain amounts of aid would be promptly forthcoming, should an oil agreement be concluded. “54 Möglich wurde eine solche Strategie aus amerikanischer Sicht durch den perzipierten Wandel der iranischen Öffentlichkeit bezüglich der Ölfrage. Anfangs als das hauptsächliche Hindernis für eine Einigung angesehen, konstatierte die CIA im Frühling 1954 freudig die zunehmend „realistische“ Sichtweise der Iraner: „In their present passive mood, the Iranian people appear prepared to accept almost any sort of agreement which makes some minimum provision for national pride. “55 Die Amerikaner führten diesen ,amazing change in Iranian attitudes“ auch auf den Erfolg der eigenen Propagandaoperationen - „both overt and covert“ - in Iran zurück. ${ }^{56}$ Der amerikanische Optimismus, die iranische Verhandlungsführung nachhaltig beeinflussen zu können, war nicht zuletzt dem Comeback des alten pro-amerikanischen Netzwerks und der Rückkehr prowestlicher und häufig an US-Universitäten ausgebildeter Technokraten in einflussreiche Positionen geschuldet. Der frühere Direktor der Nationalbank, Abu 1-Hasan Ebtehāğ, übernahm nach wiederholtem amerikanischem Lobbying die Position des Direktors der Planorganisation, die er sogleich mit westlich orientiertem Personal ausstattete. ${ }^{57}$ Der iranische Verhandlungsführer während der Ölverhandlungen, Finanzminister 'Ali Amini, galt weithin als Parteigänger der USA. Amini hatte bereits zuvor als geschickter Verhandlungspartner mit dem Konsortium erheblich an Prestige gewonnen. Mit seiner herausragenden Rolle in der anschließenden Ratifikation der Vereinbarung durch den Mağles - Amini brandmarkte Gegner des Abkommens als „Feinde Irans“ und als „,blinde Isolationisten“ - qualifizierte er sich in den Augen amerikanischer Beobachter endgültig als Kandidat für höhere Aufgaben. ${ }^{58}$ Enge sicherheitspolitische, militärische und geheimdienstliche Kooperation, wirtschaftliche Abhängigkeit und die Positionierung vertrauter Offizieller in Schlüsselpositionen, all das

\footnotetext{
${ }^{53}$ Vgl. CIA Report, encl. to Kent Memo, 29.3.54, CREST: \#RDP79*040-4; gleichzeitig warnte die CIA für das Eintreten dieses Falles vor einer Rückkehr des Chaos der Mosaddeġ-Zeit. Tatsächlich gewährten die USA weitere \$25 Millionen Wirtschaftshilfe bis zum September 1954 als Überbrückung. Vgl. OCB Progress Report, 15.4.54, ,NSC 5402‘, Policy Papers, Box 27, RG 273, NACP.

${ }_{54}$ Zugleich warnte er: "The threat must be clear that we are not prepared to carry the Iranians forever, in the absence of an oil settlement.“; im Anschluss werden zukünftige Hilfsleistungen konkretisiert, die ,immediately upon Majlis ratification" gewährt werden sollten. MemCon, 1.6.54, ,OCB 091. Iran (File \#5) (4)", WHO, NSCS, OCB Central File Ser., Box 44, DDEL \{Hervorheb. i. Orig.\}; ausgewogener noch Byroade Memo, 23.12.53, FRUS 52-54, X, S. 855f.; vgl. auch HeIss, Iranian Consortium, S. 532; FARMANFARMAIAN, Blood and Oil, S. $302 \mathrm{f}$.

${ }^{55}$ CIA/ONE, ,The Outlook in Iran', 5.3.54, CREST: RDP79*050-3.

${ }^{56}$ MemCon, 1.6.54, siehe Anm. 54; vgl. auch Punkt 7 in NSC Staff Study [Januar 1954], FRUS 52-54, X, S. 873; die verschiedenen propagandistischen Zielsetzungen sind aufgeführt in Ware Memo, 15.2.54, ,OCB 091. Iran (File \#1) (2)‘, WHO, NSCS, OCB Central File Ser., Box 42, DDEL.

57 Vgl. Milani, Eminent Persians: II, S. 739; „Ebtehaj, a strong-willed and competent man who clearly understands the need for a coordinated development plan, $[\ldots]$ he could help improve this situation, particularly if he is delegated sufficient power over development funds.“; zugleich gelte es aber ,[...] avoiding the appearance of U.S. domination or control over the use of Iranian resources [...]."; OCB Progress Report, 13.10.54, CREST: \#RDP80*001-0; die CIA verfügte bereits 1954 über Informanten innerhalb des Planungsstabes. Vgl. CIA Information Report, o.D. [August 1954], CREST: \#RDP83*001-8; ein Beispiel für Hendersons Fürsprache in Tehran \#703, 18.9.53, FRUS 52-54, X, S. 797-801.

${ }^{58}$ Henderson widersprach dem Schah, als sich dieser über US-Druck zugunsten Aminis Berufung beschwerte. Siehe Tehran \#466, 23.8.53, ebd., S. 762-5; zur US-Rezeption von Aminis Mağles-Rede vgl. Tehran \#711, 21.9.54, ebd., S. 1053f.; zur Ratifikationsdebatte vgl. HeIss, Iranian Consortium, S. 533f.; Elm, Oil, Power, and Principle, S. 326f.; zu Amini vgl. MiLANI, Eminent Persians: I, S.63-71.
} 
verschob nun die Position der USA in Iran endgültig hin zu einer dominierenden Stellung: Wenngleich die USA nicht in der Lage waren, Entscheidungsprozesse in Iran umfassend zu steuern, so konnten sie diese doch häufig maßgeblich beeinflussen. Die Beziehungen zwischen den USA und Iran glichen nun zunehmend denen einer klassischen Patron-KlientenBeziehung. ${ }^{59}$

Im Gegensatz zur vorherigen Führungsmacht Großbritannien hatten die USA aber in erster Linie keine ökonomischen, sondern zuvorderst strategische Interessen an Iran, immer im Kontext des globalen Wettbewerbs der Supermächte, der in den 1950er Jahren nun auch jenseits der bisherigen Schwerpunkte Europa und Ostasien maßgeblich wurde, wobei der Nahe und Mittlere Osten mit seinen zumindest nominell unabhängigen Staaten in dieser Dekade eine Hauptrolle spielen sollte. ${ }^{60}$ In Iran ging es Washington weiterhin prioritär um eine Stabilisierung der pro-westlichen Regierung, wobei mit der Lösung des Ölkonflikts das oberflächlich zentrale Problem nun gelöst war. Die verschiedenen Papiere und Studien seitens der intelligence community und aus dem Umfeld des zentralen Steuerungsorgans der Administration, dem Operations Coordinating Board (OCB), der den bisherigen PSB ersetzte, und für die Implementierung der NSC-Entscheidungen zuständig war, zeugen von der wachsenden Zuversicht hinsichtlich der Zukunft Irans. So revidierte die CIA eine recht pessimistische Einschätzung im Rahmen eines NIEs bereits nach wenigen Monaten, und prophezeite der Zāhedi-Regierung nun eine gute Chance, auch über das Jahr 1954 hinaus im Amt zu bleiben. Insbesondere die unerwartet reibungslos verlaufenen Wahlen zum 18. Mağles in den ersten Monaten des Jahres 1954 hatten einen Stimmungswechsel bei den amerikanischen Beobachtern herbeigeführt: ,the job of keeping the warring politicians under control may be somewhat less formidable than was suggested [...]." ${ }^{* 61}$ Allerdings illustrierten diese fast durchweg gefälschten bzw. manipulierten Wahlen auch das wachsende Misstrauen zwischen Schah Mohammad Rezā und seinem ambitionierten Ministerpräsidenten. ${ }^{62}$ Im Mai und ein weiteres Mal im Juni 1954 drängte der Schah auf eine Abberufung des ungeliebten Fazlollāh

\footnotetext{
${ }^{59} \mathrm{Zu}$ den verschiedenen Definitionen einer solchen Beziehung vgl. GASIOROwSKI, Client State, S. 1-4.

60 Dies ist nicht gleichzusetzen mit einer Preisgabe der ökonomischen Interessen. Tatsächlich war eine Aufrechterhaltung der aus westlicher Sicht günstigen Struktur der nahöstlichen Erdölökonomie sowohl im Sinne amerikanischer Konzerne wie auch strategischer Interessen der USA. Dies zeigte sich parallel in der angloamerikanischen Sabotage des sogenannten Onassis-Tanker-Deals. Vgl. 207th Meeting of the NSC, 23.7.54, DDRS: CK2349132883; CITINO, Defending.

${ }_{61}$ CIA/ONE, ,The Outlook in Iran', 5.3.54, CREST: RDP79*050-3; dies war eine Aktualisierung der Schlussfolgerung von NIE-102; vgl. CIA, NIE-102, 16.11.53, FRUS 52-54, X, S. 836f.; Eisenhower hatte nach seiner Wahl den befreundeten Bankier Robert Cutler mit einer Studie über mögliche Verbesserungen des vererbten NSC-Systems beauftragt. Unter anderem schlug Cutler die Ernennung eines Special Assistant to the President for National Security Affairs vor. Die Figur des Nationalen Sicherheitsberaters trat nun in Erscheinung, mit Cutler als erstem Inhaber der Position, wenngleich noch ohne eigenen Personalstab. Vgl. NELSON, Policy Hill; PRADOS, Keepers of the Keys, S. 61-80.

${ }_{62}$ Botschafter Henderson hatte gegenüber dem Schah die Steuerung der Wahlen explizit gebilligt, da nur so ein unabhängiger Iran erhalten werden könne. Siehe Tehran \#703, 18.9.53, FRUS 52-54, X, S. 799; Henderson selbst hatte erfolgreich auf die Auflösung von Mosaddeġs Rumpf-Mağles gedrängt. Vgl. Tehran \#866, 9.10.5, ebd., S. 810-3; zu den Spannungen zwischen Schah und Premier vgl. Tehran \#1481, 7.1.54; Tehran \#1491, 8.1.54, ebd., S. 891f., 894ff.; die Administration entschloss sich spät zu einer politischen Intervention, um auf die Kandidatenauswahl einzuwirken. Siehe das teilweise freigegebene Tehran \#1581, 18.1.54, ebd., S. 899; zu den Wahlen vgl. auch GASIOROWSKI, Client State, S. $88 \mathrm{f}$.
} 
Zāhedi - nur starker amerikanischer Druck verhinderte letztlich diesen aus Sicht Washingtons voreiligen Schritt. ${ }^{63}$

Die US-Haltung zu diesem Zeitpunkt erklärte sich in erster Linie aus Sorge um eine weitere Verzögerung der Ölverhandlungen. ${ }^{64}$ Grundsätzlich aber blieb der Erhalt einer pro-westlichen und politisch dominanten Monarchie der Schwerpunkt der US-amerikanischen Iranpolitik. Bereits unmittelbar nach dem Staatsstreich hatte Mohammad Rezā unmissverständlich klar gemacht, welche Ambitionen er hegte. So regte er den raschen Aufbau starker und loyaler Streitkräfte an, nicht nur mit Blick auf die sowjetische Bedrohung, sondern auch unverblümt als Machtmittel in der Innenpolitik. So führte er in einem Gespräch mit Loy Henderson aus, er sei bereit, gestützt auf erneuerte Streitkräfte, den Mağles aufzulösen und solange eine „Diktatur“ auszuüben, bis die Umstände wieder eine Rückkehr zu einem - seitens des Hofs gesteuerten - Konstitutionalismus erlaubten. ${ }^{65}$ US-Botschafter Henderson unterstützte dennoch nachdrücklich die Aufrüstungspläne des Schahs, wenngleich anfangs nur mit sehr begrenztem Erfolg. ${ }^{66}$ Die US-Politik fokussierte sich auf den Schah als Garanten einer proamerikanischen Ausrichtung des Landes, da das Vertrauen in die Führungsebene der Armee weiterhin gering blieb. ${ }^{67}$ In einem zweiten Besuch in den USA im Dezember 1954, dieses Mal kein offizieller Staatsbesuch wie 1949, gelang Mohammad Rezā eine weitere Aufwertung seiner ohnehin zentralen Rolle in der amerikanischen Iranpolitik. Während der Gespräche in Washington wurde Mohammad Rezā eine grundsätzliche Zustimmung zu seinen Plänen einer Aufstockung und Modernisierung der iranischen Streitkräfte signalisiert, mit dem Ziel der Transformation Irans ,from a liability to a positive asset in the Middle Eastern area“. ${ }^{6}$

Die Konzentration auf den Erhalt der Monarchie war aber nicht gleichzusetzen mit der Gewährung größeren politischen Handlungsspielraums für die iranische Führung, auch nicht

\footnotetext{
${ }^{63}$ Vgl. Tehran \#2418, 28.5.54; State \#2337, 29.5.54; State \#2343, 31.5.54; Tehran \#2513, 11.6.54, FRUS 52-54, X, S. 1012-5, 1017, $1020 \mathrm{ff} ., 1026 \mathrm{ff}$; vgl. ebd. S, $1022 \mathrm{n3}$; auch die Briten unterstützten Zāhedi gegenüber dem Schah. Die CIA war gut informiert über die vom Schah gesponserten Intrigen gegen die Zāhedi-Regierung, koordiniert vom ehemaligen Generalstabschef Hasan Arfa'. Neben dem Senator und späteren Premier Manučehr Ėgbāl waren auch die CIA-nahen Renegaten der Nationalen Front, Mozaffar Bag̀ā i und Hosejn Makki, Teil der Intrigen. Vgl. CIA Draft NSC Briefing, 18.6.54, CREST: \#RDP79*045-2.

${ }^{64}$ Die CIA warnte explizit vor einem Zusammenbruch der Verhandlungen im Falle der Abberufung Zāhedis. Siehe ebd.

${ }^{65}$ So Mohammad Rezā explizit in Tehran \#703, 18.9.53, FRUS 52-54, X, S. 799; im Dezember deutete er eine zukünftige Beteiligung an einer regionalen Verteidigungsorganisation und eine offene Parteinahme für den Westen im Kalten Krieg an. Vgl. Tehran \#1229, 3.12.53, FRUS 52-54, IX, S. 436ff.

${ }_{66}$ Im Anschluss an NSC 5402 entschied der NSC im Februar 1954 die Gewährung zusätzlicher \$25 Millionen Militärhilfe, aus Sicht der Befürworter einer iranischen Aufrüstung eine enttäuschende Größenordnung. Vgl. State \#1751, 19.2.54, FRUS 52-54, X, S. $928 \mathrm{ff}$.

${ }^{67}$ Der OCB empfahl vorerst auf eine gesetzliche Regelung für die Thronfolge in Iran, wie angeregt in NSC 5402, zu verzichten, da bereits eine solche Debatte konkurrierende Prätendenten aus der breiteren Pahlawi-Familie stärken könnte. Siehe OCB Progress Report, 13.10.54, CREST: \#RDP80*001-0; vgl. Stevens to Eden, No. 93, 16.11.54, FOCP V, B, 7, S. 107f.; groß war die Skepsis gegenüber den Streitkräften, ,whose political outlook cannot be clearly determined and whose officers constitute by no means a united corps [...]."; Howe Memo, 30.7.54, FRUS 52-54, X, S. 1041.

68 Jernegan Memo, 9.12.54, ebd., S. 1067; die iranischen Streitkräfte sollten mittelfristig zu ,defensive delaying capabilities“ im Falle eines sowjetischen Angriffs befähigt werden. Zu den, wenngleich noch unverbindlichen, Zusagen der US-Offiziellen vgl. State \#1187, 15.12.54, ebd., S. 1076f.; Shah Memo, encl. to Eisenhower Memo, 14.12.54, ,Iran, 1953 through 1959 (7)‘, AWF, International Ser., Box 32, DDEL; zum Besuch vgl. auch GOODE, United States and Iran, S. $152 \mathrm{f}$.
} 
für Mohammad Rezā selbst. Die gewaltige Asymmetrie zwischen dem amerikanischen Patron und dem iranischen Klienten kam nun immer mehr zum Tragen. Ein OCB-Bericht vom Oktober 1954 betonte die entscheidende Rolle der finanziellen Unterstützung durch die USA seit dem Staatsstreich, welche erst die für eine Einigung mit den Briten notwendige wirtschaftliche Stabilisierung und kompromissbereite Atmosphäre geschaffen habe. Tatsächlich finanzierten die USA ein monatliches Budgetdefizit von \$5 Millionen - Mark Gasiorowski zufolge deckte US-Unterstützung im Finanzjahr 1954 etwa 60\% der staatlichen Ausgaben, was das Ausmaß der iranischen Abhängigkeit nachdrücklich illustriert. ${ }^{69}$ Trotz der rasch steigenden Öleinnahmen - erwartet wurden bereits für 1955 \$60 Millionen - war man sich in der Eisenhower-Administration bewusst, dass Iran auch in den Folgejahren stark von amerikanischen Transferleistungen abhängig sein würde, , $[\ldots]$ to cover politically necessary increases in budgetary expenditures and projected development programs. “70 Unmittelbar nach Abschluss der Konsortiumsvereinbarung, aber noch vor der Ratifikation durch den Mağles, beschlossen die USA großzügige Wirtschaftshilfe für das nächste Finanzjahr in Höhe von \$117 Millionen in Form von Zuschüssen und Darlehen. Allerdings drängte die EisenhowerAdministration nun massiv auf amerikanische Beteiligung und Supervision an der politischen Entscheidungsfindung über die Verwendung dieser Gelder. Zudem war die Auszahlung an diverse iranische Konzessionen, wie z.B. Importeinschränkungen und Verzicht auf monetäre Maßnahmen zur Aufwertung des Rial, gebunden. Darüber hinaus regte Washington die Übertragung der Ausgabenkontrolle an ein Finanzkomitee dominiert von den amerikanischen Parteigängern Amini und Ebtehāğ an, um die alleinige Verwendung für Entwicklungsabsichten zu garantieren, im Grunde eine Art Schattenregierung, kontrolliert durch die USA. ${ }^{71}$

Mit dem umfangreichen Hilfsprogramm wuchs der Einfluss der USA in vielen gesellschaftlichen und wirtschaftlichen Bereichen. Amerikanische Universitäten berieten iranische Stellen in Fragen öffentlicher Verwaltung und landwirtschaftlicher Verbesserungen, US-Berater halfen bei der Neugestaltung des Gesundheitswesens, einer Einkommenssteuerreform und bei der Erstellung des ersten nationalen Zensus. Das iranische Bildungssystem wurde von Grund auf reformiert, ,along American lines“. ${ }^{2}$ Ebtehāğ setzte zuvorderst auf amerikanische und europäische Berater - 1958 waren etwa 30 Consulting-

\footnotetext{
${ }^{69}$ Vgl. OCB Progress Report, 15.4.54, ,NSC 5402‘, Policy Papers, Box 27, RG 273, NACP; OCB Progress Report, 13.10.54, CREST: \#RDP80*001-0; GASIOROWSKI, Client State, S. 101f.

${ }^{70}$ OCB Progress Report, 13.10.54, CREST: \#RDP80*001-0; die Öleinnahmen entsprachen zwar ungefähr der bisherigen US-Budgethilfe, doch rechnete man im Anschluss an das Ölabkommen mit Ausgabensteigerungen von ca. $35 \%$ für politische notwendige Gehaltsaufbesserungen von Staatsbediensteten und Soldaten.

${ }^{71}$ Etwa \$22 Millionen der Summe waren Zuschüsse. Vgl. Joint State-FOA Telegram, 13.10.54; Tehran \#874, 15.10.54; State \#742, 15.10.54, FRUS 52-54, X, S. 1057ff., 1059f., 1061; die Zāhedi-Regierung akzeptierte die Bedingungen. Siehe ebd., S. 1061 n3; \$18 Millionen der Summe waren bestimmt für die Fertigstellung des KarağStaudamms. Zur Bedeutung dieses Schlüsselprojekts für die iranische Entwicklungspolitik und die Entstehung einer auf Massenkonsum basierten Wirtschaftsform und die Steigerung des Lebensstandards vgl. SCHAYEGH, Karaj Dam Affair.

${ }^{72}$ Vgl. OCB Progress Report, 13.10.54, CREST: \#RDP80*001-0; ein CIA-Dokument erwähnt auch eigene Studien zu einer möglichen Landreform in Iran. Vgl. Trezise Memo, 15.3.54, CREST: \#RDP69*015-8; ernüchternde Beispiele für die Ineffizienz der amerikanischen Entwicklungshilfe auf dem Land in EMBRY, Point Four, S. 108f.; vgl. auch SHANNON, Education.
} 
Unternehmen in Iran tätig, welche die verschiedenen Entwicklungsprojekte überwachten. Der Zweite Siebenjahresplan (1955-62) legte einen Schwerpunkt auf regionale Entwicklung, insbesondere in der südlichen Provinz Xuzestān. Das Vorzeigeprojekt einer integrierten regionalen Entwicklungsstrategie in der Provinz inklusive überaus ambitionierter Teilprojekte für Bewässerungsanlagen, Dammbau und Wasserkraftwerke wurde von der amerikanischen Ingenieurs- und Beratungsgesellschaft Development and Resources Corporation ausgeführt, geleitet von David Lilienthal, dem ehemaligen Vorsitzenden der Tennessee Valley Authority aus der Zeit des New Deal, der die Erfolge dieser Unternehmung nun auf die iranische Peripherie anzuwenden gedachte. Lilienthal war fortan ein häufiger Gast in Iran und wurde auch ein enger Vertrauter des Monarchen und später Gründungsmitglied des informellen, in den USA für die Unterstützung der iranischen Dynastie eintretenden Netzwerkes der „Pahlavists“. ${ }^{73}$

Mit dem gewachsenen eigenen Einfluss wurde nun auch ein zunehmendes Interesse an den sozialen und politischen Strukturen des Landes spürbar - es wurden erste Stimmen laut, die für eine erfolgreiche Umsetzung des strategischen Ziels eines langfristig stabilen und unabhängigen Irans umfassende Reformen für unabdingbar hielten. In einer Vorwegnahme der für die nächste Dekade handlungsleitend werdenden Interpretation der iranischen Reformhindernisse diagnostizierte man die ,[...] historical concentration of the control of political and economic activities in a comparatively small number of leading families who have consistently, although disunitedly, resisted the efforts of politically ambitious urban middle class elements to broaden participation in the control of these activities." Diese urbanen Mittelschichten aber ließen sich aufgrund der gegenwärtigen Schwäche und fehlenden Legitimität der politischen Institutionen in Iran nicht kooptieren. Der neue Mağles sei unerfahren und undiszipliniert - das Misstrauen des Schahs ließ zudem einen starken Premier mit einem erfolgreichen und populären Reformprogramm nicht zu. ${ }^{74}$

Vorerst aber dominierte die Sichtweise, derzufolge die Etablierung weitreichenden amerikanischen Einflusses auf iranische Politikformulierung und die Verbreitung amerikanischer Entwicklungs- und Problemlösungsansätze mittelfristig entsprechende Reformen evozieren würden. Angesichts der Bedeutung der Streitkräfte für die Aufrechterhaltung einer pro-westlichen Orientierung in den Tagen des Staatsstreichs und der einseitigen Betonung des Militärischen im Denken Mohammad Rezās überrascht die amerikanische Fokussierung auf dieses Feld im Nachhinein nicht. Die JCS hatten die zentrale

\footnotetext{
${ }^{73}$ Siehe den Einträge vom 16.9.55, 3.3.56, in: LiLIENTHAL, Journals: $I V$, S. 26f., 80f.; zu den Aktivitäten der Development and Resources Corporation in Xuzestān ab 1956 vgl. BostOCK/JONES, Planning and Power, S. 1338; AfKHAMI, Life and Times, S. 209f.; BILL, Eagle and Lion, S. 120-4; FISHER, Lilienthal; GoODELL, Rural Development in Pahlavi Iran; zur Vorbildfunktion der Tennessee Valley Authority und zu Lilienthal vgl. EKBLADH, Profits of Development.

${ }^{74}$ Vgl. OCB Progress Report, 15.4.54, ,NSC 5402‘, Policy Papers, Box 27, RG 273, NACP; vgl. dagegen die weitaus stärkeren reformistischen Absichten gegenüber den arabischen Staaten in der zentralen Direktive der Truman-Administration. Siehe NSC Staff Study (NSC 129/1), 24.4.52, „Near East (4)“, WHO, NSCS, Disaster File Ser., Box 64, DDEL.
} 
geographische Lage Irans als „blocking position“ gegen einen sowjetischen Vorstoß in Richtung Türkei, Pakistan, Persischen Golf oder Suezkanal immer wieder betont, sich aber zugleich gegen verpflichtende Beistandszusagen gegenüber Staaten wie Iran zur Wehr gesetzt. ${ }^{75}$ Ausschlaggebend waren dabei die bestehenden Unklarheiten über den Nutzen einer regionalen Verteidigungsorganisation mit anglo-amerikanischer Unterstützung und die mögliche Rolle Irans in einer solchen. In der Zwischenzeit wurde der Schah mit einer begrenzten Zusage von weiteren \$25 Millionen Militärhilfe abgespeist, sowie der Zusage eines Ausbaus der amerikanischen Beratermissionen. ARMISH, bald verschmolzen mit der nach 1950 zusätzlichen eingerichteten Military Assistance Advisory Group (MAAG) zu ARMISHMAAG, wurde nun vergrößert und Henderson gelang es in der Folgezeit, die Bedenken in Washington gegen eine Ausweitung der Militärhilfe für Iran zu mindern. ${ }^{76}$ MAAG Chief Brigadegeneral Robert A. McClure hatte in der Zwischenzeit nach Konsultationen mit iranischen militärischen Stellen einen Dreijahresplan für eine umfassende Neuausrichtung und Modernisierung der Streitkräfte ausgearbeitet. Die Kosten für den McClure-Plan wurden auf \$360 Millionen taxiert, eine für frühere Planungen kaum vorstellbare Größenordnung, was vermutlich auch die Weigerung des DOD erklärt, sich ernsthaft mit dem Plan auseinanderzusetzen. ${ }^{77}$ Zwischen DOD und JCS auf der einen und dem State Department auf der anderen Seite entstand nun ein bürokratischer Konflikt über Zielsetzung und Größenordnung des iranischen Militärhilfeprogramms in der Zukunft. Vorerst aber überzeugten die Bedenken der militärischen Seite, wonach ohne Verabschiedung einer umfassenden Strategie über die Verteidigung der Gesamtregion und über amerikanische Unterstützung für eine Organisation der regionalen Staaten zu diesem Zweck jede Festlegung auf den Beitrag Irans verfrüht sei. ${ }^{78}$

\footnotetext{
75 „Blocking position“ in NSC Staff Study, 21.12.53, ,Annex to NSC 175`, Policy Papers, Box 26, RG 273, NACP; hinzu kam die herausragende Bedeutung der Ölförderung am Golf. Dieselbe Studie erwähnte Projektionen, dass Westeuropa im Jahr 1975 zu wenigstens 90\% von nahöstlichem Erdöl abhängig sein würde. Die JCS hatten sich noch im September 1954 gegen einen voreiligen Entscheid zugunsten einer Aufrüstung Irans gewandt, solange keine endgültige Entscheidung bezüglich einer regionalen Verteidigungskollaboration gefällt worden sei. Vgl. OCB Progress Report, 13.10.54, CREST: \#RDP80*001-0, S. 12; Bishop Memo, 14.9.54, , OCB 091. Iran (File \#1) $(8)^{\circ}$, WHO, NSCS, OCB Central File Ser., Box 43, DDEL.

76 Zur zusätzlichen Militärhilfe vgl. State \#1751, 19.2.54, FRUS 52-54, X, S. 928ff.; obgleich es noch keine amerikanische Festlegung auf die Verteidigung der Pässe im Zāgros-Gebirge gab, hatte man die Entsendung zusätzlicher Trainingsteams mit 65 Offizieren und 125 weiteren Soldaten unterhalb der Kommandoebene zugesagt. Die Militärmissionen wurden vorerst auf Basis des unter Mosaddeg etablierten „Gentlemen's Agreement“ verlängert. Vgl. OCB Progress Report, 13.10.54, vorherig. Anm.; JCS Memos, 12.2.52 und 19.3.52, in: AleXAnder/NANeS, United States and Iran, S. 237-9; zu Hendersons Bemühungen vgl. Tehran \#1102, 14.11.53, FRUS 52-54, X., S. 831-4; mit ein Grund für das Zögern auf US-Seite war das Bestreben, Mohammad Rezās Drängen auf einen Ausbau der Streitkräfte an seine Unterstützung der Zāhedi-Regierung und eines Ölabkommens zu koppeln. Siehe vor allem Hendersons Kommentar in ebd., S. 834; sowie die Diskussion im NSC in 199th NSC Meeting, 27.5.54, ebd., S. 1010; vgl. auch Tehran \#1872, 4.3.54; Tehran \#1972, 18.3.54, ebd., S. 934-40, 954-8; vgl. JCS History Iran, S. 36f.

${ }^{77}$ Vgl. Jernegan Memo, 18.9.54; Dulles Memo, 8.11.54, FRUS 52-54, X, S. 1052f., 1063-6; die JCS akzeptierten allerdings McClures Vorschläge für eine Neugliederung der Streitkräfte in 8 leichte Infanteriedivisionen, 4 leichte gepanzerte Divisionen plus 5 unabhängige Infanteriebrigaden. Dies ging bereits über die 1951 für Iran veranschlagte Sollstärke von ca. 135.000 Mann hinaus und lag nahe an den von Mohammad Rezā gewünschten 150.000. Die Neugliederung hatte bereits 1956 Bestand. Vgl. JCS History Iran, S. 39, 54; vgl. JCS Memo, 12.10.54, in: ALEXANDER/NANES, United States and Iran, S. $270 \mathrm{ff}$.

${ }_{78}$ Vgl. beispielhaft Jernegan Memo, 7.1.55; JCS Memo, 7.1.55, FRUS 55-57, XII, S. 680ff.: „The Joint Chiefs have always jumped behind the British skirts and have not adopted or recommended an independent American plan for that part of the world, in spite of the fact that we have NSC approval of the 'northern tier concept' and of
} 
Bereits Ende der 1940er Jahre war die Vernachlässigung der Nahostregion im Rahmen der globalen Eindämmungsstrategie mehr und mehr unter Beschuss geraten, ebenso aber der vermeintliche Königsweg einer einseitigen Konzentration auf die unmittelbaren „Frontstaaten“ entlang des Northern Tier. ${ }^{79}$ Angesichts der bis 1950 nur begrenzt zur Verfügung stehenden Ressourcen war man insbesondere auf Seiten des amerikanischen Militärs anfangs erpicht, keine weiteren Verpflichtungen in der Nahostregion einzugehen, zumal die erhöhte Reichweite der strategischen Bomber die bisherige räumliche Abhängigkeit auf das Kairo-Suez-Gebiet als Ausgangspunkt für einen nuklearen Angriff auf die UdSSR obsolet gemacht hatte. ${ }^{80}$ Umso mehr hatte man daher auf amerikanische Seite ein Interesse an einer fortdauernden britischen Verantwortlichkeit für die Region des Nahen und Mittleren Ostens. Die sogenannten McGheeWright-Gespräche vom November 1949 hatten diese ein weiteres Mal betont, die Abmachungen der Pentagon-Gespräche von 1947 im Grundsatz bestätigend. ${ }^{81}$ Mit der Herabstufung der strategischen Bedeutung der Nahostregion durch die US-Planer - im Januar 1950 bezeichneten die JCS nunmehr die eigenen militärstrategischen Interessen in der Region als ,,almost negligible“ im Vergleich zu anderen Weltregionen - setzte aber bald ein, anfänglich politisch motiviertes Umdenken innerhalb des State Department ein, aus dem dann u.a. die insbesondere von McGhee geforderte Initiative zur Ausdehnung der Militär- und Wirtschaftshilfeprogramme auf die nahöstlichen und südasiatischen Staaten hervorgegangen war. ${ }^{82}$ Der Ausbruch des Koreakrieges wirkte alsbald katalysierend auf diese verschiedenen Konzeptionen und Initiativen bezüglich einer Regionalisierung der Verteidigungsanstrengungen in der Nahostregion.

Angesichts britischer Schwäche und amerikanischer Zurückhaltung wurde in den verschiedenen Kriegsplänen zuerst die Rolle regionaler Staaten aufgewertet, insbesondere die der Türkei. ${ }^{83}$ Gegen anfänglich starken Widerstand der JCS setzte vor allem nun das State Department - und hier wieder vor allem George McGhee - eine Ausweitung amerikanischer Sicherheitsgarantien auf das östliche Mittelmeer durch. Nach langwierigen internen Auseinandersetzungen billigten im Mai 1951 sowohl der NSC wie auch Präsident Truman den Beitritt der Türkei - wie auch logisch folgend denjenigen Griechenlands - zur NATO. ${ }^{84}$ Nachdem auch die britischen Verbündeten ihr Beharren aufgegeben hatten, zuvor eine

the importance of the entire Middle East", beschwerte man sich im NSC-Stab. Bishop Memo, 14.9.54, ,OCB 091. Iran (File \#1) (8)‘, WHO, NSCS, OCB Central File Ser., Box 43, DDEL.

${ }_{79}$ Vgl. Merriam Memo, 13.6.49, FRUS 49, VI, S. 34; Überblick bei KuniHolm, Rings and Flanks, S. $117 \mathrm{ff}$.; COHEN, Strategic Role; DeVEREUX, Formulation, S. 19-24.

${ }^{80}$ Vgl. Cohen, Fighting World War Three, S. 46ff., 90f.; vgl. Aldrich/ZAMETICA, Rise and Decline, S. 263f.; DeVEreuX, Formulation, S. 23f.; OnOzawA, American Regional Policy, S. 119-22.

${ }^{81}$ Vgl. Agreed Memorandum, 25.7.50, FRUS 50, V, S. 188-191; für eine Übersicht über die verschiedenen angedachten Verteidigungsringe bzw. -positionen in der Region vgl. COHEN, Fighting World War Three, S. 1637, insbes. die Karte auf S. 164f.; vgl. HAHN, Middle East Command, S. 27f.; vgl. ALDRICH/ZAMETICA, Rise and Decline, S. 258-62.

${ }^{82}$ „Almost negligible“ in Bray Memo, 25.1.50, FRUS 50, V, S. 122f., $123 n 6$.

83 Vgl. Kuniholm, Defense of the Middle East, S. 121f.; LEFFLER, Strategy, Diplomacy, S. $817 \mathrm{ff}$.

${ }^{84}$ Vgl. McGhee Memo, 18.4.51, ,General-Top Secret', Lot 53 D 468, Papers of McGhee, Box 11; zum Umdenken der JCS vgl. COHEN, Fighting World War Three, S. 58-60. 
Beteiligung Ankara an einer rein regionalen Verteidigungsorganisation durchzusetzen, war der Weg zur Aufnahme der beiden Länder in die Allianz frei, die schließlich im Februar 1952 erfolgte. ${ }^{85}$ Mit der Ausdehnung des NATO-Bündnisses aufs östliche Mittelmeer, initiiert und durchgesetzt von der amerikanischen Führungsmacht, und der Übernahme sicherheitspolitischer Verpflichtungen in der Region seitens der USA wurde im Kern die Botschaft der Truman-Doktrin von 1947 ein weiteres Mal bestätigt und nunmehr auch durch formale Beistandsgarantien konkretisiert. Diese Konkretisierung brachte allerdings auch eine grundlegende Wende der amerikanischen Nahostpolitik mit sich: Angesichts britischer Ressourcenschwäche musste die bisherige arbeitsteilige Ausfüllung des ContainmentKonzeptes in Zusammenarbeit mit Großbritannien revidiert werden und ein verstärktes Engagement der USA war somit unumgänglich. Hinzu kam die wachsende Erkenntnis, dass die Briten trotz aller Erfahrung und Expertise nicht in der Lage waren, die Entwicklungen in der Nahostregion zum Vorteil westlicher Interessen zu steuern. Jahrhundertelange Erfahrung im Umgang mit den indigenen Bevölkerungen halfen wenig angesichts des, so ein US-Admiral im Mai 1951, ,[...] shrewd estimate by these countries that the U.K. is no longer a great Power." ${ }^{86}$

Nahöstliche Stabilität sollte nun nicht mehr ausschließlich auf dem - seitens der USA akzeptierten und unterstützten - Primat Großbritanniens im Sinne des latenten angloamerikanischen Kondominiums fußen. Von nun an engagierte sich Washington notgedrungen stärker als Ordnungsmacht in der Region und steigerte daher ökonomische wie militärische Unterstützungsleistungen. ${ }^{87}$ Mit der Übernahme größerer Verantwortung zeigte sich die Truman-Administration zudem aufgeschlossener gegenüber der bislang ablehnend beschiedenen Idee einer kollektiven Verteidigung der Nahostregion. In London hoffte man auf eine Beteiligung der USA an dem angedachten Middle East Command (MEC), verlautbart im September 1951, mit dem Ziel einer Erneuerung der britischen Position mittels vertraglicher Regelungen über die Nutzungs- und Zugangsrechte der zentralen Suez-Basis mit Ägypten eine Hoffnung, die sich allerdings nicht erfüllen sollte. ${ }^{88}$

\footnotetext{
${ }^{85} \mathrm{Zu}$ den anglo-amerikanischen Beratungen vgl. PoOLE, History of the JCS: $I V$, S. 120-5.

${ }^{86}$ So der Chief of Naval Operations Admiral Forrest Sherman in State-JCS Meeting, 2.5.51, FRUS 51, V, S. 115; selbst McGhee musste eingestehen, die Unpopularität der Briten könnte zu einer „Belastung“ in einem Maße werden, ,that it may exceed the military value of cooperating with them in the area."; ebd., hier 114, 115 ; vgl. auch LUCAS/TAKEYH, Alliance and Balance, S. $635 \mathrm{ff}$.

${ }_{87}^{87}$ Laut McGhee verlangte es nach einer neuen Richtung der US-Politik, weg ,[...] from the concept of primary British responsibility and toward the concept of combined US-UK responsibility and active US-UK cooperation in the development and implementation of plans."; Annex ,Re-Evaluation of US Plans for the Middle East", o.D., 27.12.50, FRUS 51, V, S. 11; NEA Memo ,The Middle East‘, o.D., ebd., S. 24-27.

${ }^{88}$ Vgl. McGhee Memo, 6.12.50, FRUS 50, V, S. 326ff.; ed. note in FRUS 51, III, S. 522ff.; zu den Verhandlungen über das MEC vgl. CoHEn, Fighting World War Three, S. 239-72; OnOZAWA, American Regional Policy, S. 13036; PoOLE, History of the JCS: IV, S. 139-42; LuCAS, Divided We Stand, S. 5-11; DevereuX, Collective Defence; zur Vorbildfunktion der Nahostgespräche von 1947 und 1949 für die Aufrechterhaltung der special relationship aus britischer Sicht vgl. US Delegation \#6, 24.4.50, FRUS 50, III, S. 854ff.; siehe auch die Bemerkungen zu "special bipartite machinery" und den Hinweis auf bereits existierende, ,ultra-secret global planning arrangements" in U.K. Delegation Brief, 21.4.50, DBPO II, II, S. 74, 75; Agreed Anglo-American Report, 6.5.50, ebd., S. 242ff.; vgl. ACHESON, Present at the Creation, S. $387 \mathrm{f}$.
} 
Vor allem dem bürokratischen Widerstand der Militärführung war es zuzuschreiben, dass McGhees Ideen einer neuen und bedeutenden Rolle für die USA in der gesamten Nahostregion letztlich nur eine bescheidene Umsetzung erfuhren. Neben der Weigerung, weitere und angesichts der bestehenden Belastungen ohnehin kaum zu erfüllende Verpflichtungen zu übernehmen, war es seitens der JCS auch gestiegenes Misstrauen gegenüber den britischen Motiven, die ein stärkeres US-Engagement im Fahrwasser der vormaligen Hegemonialmacht als wenig attraktiv erscheinen ließen. ${ }^{89}$ Die Gestaltung des MEC zu einem nahöstlichen Pendant zur NATO einschließlich amerikanischer Sicherheitsgarantien war aus Sicht der USMilitärführung schlicht inakzeptabel. ${ }^{90}$ McGhees Ideen einer Konkretisierung des bislang nur latenten anglo-amerikanischen Kondominiums im Nahen und Mittleren Osten in eine „,combined US-UK responsibility“ für die Region widersprach zudem der etablierten USKonzeption der special relationship, die jeder Institutionalisierung im Grundsatz ablehnend gegenüberstand. ${ }^{91}$ Das unter großem diplomatischen Aufwand konzipierte MEC scheiterte alsbald an den regionalen Realitäten, insbesondere am aufstrebenden ägyptischen Nationalismus. Amerikaner und Briten weigerten sich aber, dies zu akzeptieren und legten in der Folgezeit revidierte Fassungen des Plans vor. ${ }^{92}$ Überhört wurden dabei warnende Stimmen innerhalb des State Department hinsichtlich der grundsätzlichen Realisierbarkeit regionaler Verteidigungsinitiativen. ${ }^{93}$

Aus US-Sicht traten die militärstrategischen Überlegungen einer fortgesetzten Kooperation mit den Briten immer mehr in den Hintergrund. Bedeutender war nun die fortgesetzte Bereitschaft

\footnotetext{
${ }^{89}$ Londons Bemühungen um einen britischen NATO-Oberbefehl im östlichen Mittelmeer erschienen aus Sicht der US-Militärs als ein Versuch ,[...] to upset and to reverse the developments of the last six years which have resulted in our acquiring the dominant military position in the area"; State-JCS Meeting, 14.3.51, FRUS 51, III, S. 490f. ${ }^{90}$ Vgl. ,Draft Anglo-United States Agreement', encl. to Ferguson Memo, 18.7.51, FRUS 51, III, S. $562 \mathrm{ff} .$.

${ }^{91}$ Für Kritiken der Ideen McGhees vgl. Thayer Memo, 12.2.51, FRUS 51, V, S. 48f.; Cabot Memo, 19.2.51, ebd., S. 78f.; sowie die Antwort in Berry Memo, 21.2.51, ebd., S. 83-7; letztlich resultierten all diese Bemühungen in der Studie NSC 47/5 vom März 1951, die eine moderate Revision der bisherigen Politik gegenüber den arabischen Staaten und Israel mittels eines limitierten Militärhilfeprogramms einleiten sollte. Vgl. NSC 47/5, 14.3.51, ebd., S. 95-7; eine umfassende Hasbara-Kampagne der israelischen Regierung zwang die Administration, die veranschlagten Hilfslieferungen an Israel auf eine Summe heraufzusetzen, die derjenigen für alle arabischen Empfängerstaaten zusammen entsprach. Vgl. HAHN, Caught, S. 79-82; der arabisch-israelische Konflikt sabotierte somit im Kern die Bemühungen McGhees zur Errichtung eines anti-sowjetischen Verteidigungsbündnisses in der Nahostregion.

${ }_{92}$ Noch vor der diplomatischen Offerte an Ägypten, vollwertiges Mitglied des MEC zu werden, hatten sich bereits die USA, Großbritannien, Frankreich und die Türkei zur Teilnahme verpflichtet. Zudem gab es eine Einladung zur Zusammenarbeit an die Commonwealth-Staaten Australien, Neuseeland und Südafrika, die ihrerseits die Bereitstellung von Truppen zugesagt hatten. Wichtigstes Ziel des MEC-Konzeptes war es, doch noch eine vertragliche Regelung über die Nutzungs- und Zugangsrechte der zentralen Suez-Basis Großbritanniens zu ermöglichen - letztlich beschleunigte das MEC aber den Verlust der britischen Position im Oktober mit der Kündigung des Anglo-Ägyptischen Vertrags von 1936 und auch des Kondominiums über Sudan durch Kairo. Vgl. Devereux, Collective Defence, S. 242-5; CoHEn, Fighting World War Three, S. 270-2, 284-7; HAHN, Middle East Command, S. 31f., 34-8; ders., United States, Great Britain, and Egypt, S. 114ff., 122-9; ONOZAWA, American Regional Policy, S. $131 \mathrm{ff}$;; PoOLE, History of the JCS: IV, S. 171-6; vgl. Matthews Memo to Lovett, 31.10.51, FRUS 51, V, S. 238-43; Ägypten förderte alternativ den in Vergessenheit geratenen Arab League Collective Security Pact, 1950 unterzeichnet und 1952 in Kraft getreten. Vgl. PERSSON, Baghdad Pact, S. 80-4.

${ }^{93}$ Der Direktor des aus dem bisherigen Office of African and Near Eastern Affairs (ANE) hervorgegangenen Office of Near Eastern Affairs (NE), G. Lewis Jones, warnte, dass eine von externen Mächten oktroyierte Verteidigungsstruktur auf den Widerstand der regionalen Akteure stoßen würde. Sinnvoller wäre es genuine regionale Tendenzen im eigenen Sinne zu nutzen: „,roll with the nationalism punch“, wie Jones es ausdrückte. Siehe U.S. Minutes, 19.6.51, FRUS 51, III, S. 542; ähnlich auch die Kritik der US-Geheimdienste. Vgl. CIA, NIE26, 25.4.51, CIA-FOIA; CIA, SE-23, 17.3.52, FRUS 52-54, IX, S. 195-9.
} 
Großbritanniens, eine weiterhin tragende und die westlichen Interessen unterstützende globale Rolle zu spielen. Die militärische Unfähigkeit Großbritanniens, im Ernstfall entscheidend zur Verteidigung der Nahostregion beizutragen, gedachten die amerikanischen Planer in erster Linie durch einen weiteren Ausbau der militärischen Kapazitäten der Türkei und mittelfristig durch eine stärkere Einbeziehung Pakistans, dessen möglicher Beitrag in erster Linie aus demographischen Gründen interessant war, zu kompensieren. ${ }^{94}$ Hier lag auch der Ursprung der späteren Konzentration der Planungen für die regionale Verteidigung auf die Staaten des Northern Tier, bald verwirklicht unter der Eisenhower-Administration. ${ }^{95}$ Allerdings waren sich Briten wie Amerikaner durchweg bewusst, dass die Grundannahme hinter all diesen Anstrengungen zugunsten einer regionalen Verteidigung auf recht zweifelhaften Prämissen beruhten. Ungeklärt war insbesondere, warum für den Kriegsfall überhaupt von einer sowjetischen Invasion der Nahostregion ausgegangen wurde, da auch Moskau mit der rechtzeitigen Zerstörung der Ölförderanlagen und der Raffinerien rechnen musste. Winston Churchill, im Oktober 1951 in das Amt des britischen Premierministers zurückgekehrt, befand die Idee einer , ,...] heavy Russian invasion of the Middle East and across the Suez Canal into Egypt, Libya, Cyrenaica and Tripoli“ für absurd. ${ }^{96}$

Angesichts der wachsenden Zweifel am Nutzen und der Realisierbarkeit eines nahöstlichen Pendants zur NATO überrascht das Scheitern des Nachfolgeprojekts zum MEC, das Konzept einer Middle East Defence Organization (MEDO), wenig. ${ }^{97}$ Im Kern eine ausgedünnte Variante des Vorgängers, nunmehr nur noch als Planorganisation und nicht mehr als vollwertiger Kommandobereich mit Anbindung an die NATO konzipiert, nunmehr beheimatet in einem anvisierten Hauptquartier auf Zypern statt in der politisch heiklen Suez-Basis, schien der neue Plan besser angepasst an die politischen Realitäten im noch jungen nahöstlichen Staatensystem und angesichts der nationalistischen Befindlichkeiten der dortigen Bevölkerungen. Wie der MEC scheiterte auch MEDO letztlich an Entwicklungen in der ägyptischen Innenpolitik - im Juli 1952 ergriffen die „Freien Offiziere“ in einem Staatsstreich

\footnotetext{
${ }^{94}$ Vgl. CINCNELM and Cs-in-C, Middle East Memo, 13.3.51, FRUS 51, V, S. 94f.; siehe auch ebd., S. 28 n5; Ankara \#994, 31.3.52, FRUS 52-54, IX, S. 199-203; vgl. COS Committee Memo, 18.12.51, BDEE B, 4, II, S. 290305; PoOLe, History of the JCS: IV, S. 191ff.; COHEN, Fighting World War Three, S. 274ff.; DEVEREUX, Formulation, S. 105ff.

${ }_{95}$ Paul Nitze hatte bereits im Frühling 1952 eine solche Entwicklung vorweggenommen, als er die eher virtuellen MEDO-Planungen zugunsten einer formellen Ausdehnung der Truman-Doktrin auf Iran verwarf und alternativ ,[...] the development in that country of a really effective barrier to Soviet expansion“ vorschlug; MemCon, 24.4.52, FRUS 52-54, IX, S. 220; die strategische Bedeutung, die inzwischen der Türkei beigemessen wurde, und die sich daraus ergebenden politischen Veränderungen spiegelte ein Planungspapier über das MEC vom Sommer 1951 wider: „In light of the increasing decline of British prestige [...] in first instance primary reliance for ground forces for the defense [...] must be placed in the Turks."; Ferguson Draft Memo, 6.7.51, FRUS 51, III, S. 554; im April 1952 plädierten die JCS sogar dafür, Ankara zur Annahme einer ,primary leadership among the nations in the area" mit dem Ziel des Aufbaus einer effektiven regionalen Verteidigung zu bewegen. Vgl. JCS Memo, 15.4.52, "Near East (3)“", WHO, NSCS, Disaster File Ser., Box 64, DDEL.

${ }_{96}$ Churchill to Alexander, 3.4.52, CAB 21/3497, TNA:PRO; vgl. auch Churchill Letter to Eisenhower, 21.6.54, in: BOYLE, Churchill-Eisenhower Correspondence, S. 147ff.; sämtliche militärischen Planungen gingen von einer groß angelegten Blitzoffensive der Roten Armee in der Nahostregion unmittelbar nach Kriegsausbruch aus. Vgl. CIA, NIE-3, 15.11.50, CIA-FOIA, hier S. 10; vgl. CIA, Intel Memo No. 263, 20.1.50, CIA-FOIA; vgl. auch COHEN, Fighting World War Three, S. 162f.

${ }^{97}$ Zur MEDO vgl. HAHn, Middle East Command, S. 38ff.; CoHEN, Fighting World War Three, S. 292-7; PODEH, Quest for Hegemony, S. 55-9; vgl. Acheson Memo, 29.12.51, FRUS 51, V, S. $265 \mathrm{ff}$.
} 
die Macht in Kairo. Der ohnehin bestehende Einfluss des Schlüsselstaates Ägyptens auf die öffentliche Meinung in den arabischen Staaten sollte sich alsbald in potenzierter Ausprägung in eine regelrechte (kulturelle) Dominanz ausweiten. Unter dem charismatischen Führer des panarabischen Nationalismus Gamal Abd-an-Nasser (Ğamāl 'Abd an-Nāṣir), ab 1954 als Ministerpräsident und ab 1956 als Präsident, stieg Ägypten als revolutionäre Republik zu einer der führenden blockfreien Mächte und zu einem bestimmenden Faktor der nahöstlichen Politik auf, dessen konsequenter Anti-Kolonialismus die Bemühungen zugunsten der Etablierung eines pro-westlichen Militärbündnisses in der Region stark behinderte. Mit Bezug auf MEDO hatte der neue britische Außenminister Anthony Eden vergeblich gehofft, ,[...] to persuade the United States to assume the real burdens in such organisations while retaining for ourselves as much political control - and hence prestige and world influence - as we can.“98

In der Tat wuchs auf amerikanischer Seite die Bereitschaft zu einem stärkeren Engagement in der nahöstlichen Weltregion. NSC 129/1 vom April 1952 sprach von einem, increased share of responsibility toward the area“ und erwähnte zumindest die Möglichkeit „token U.S. forces“ als eigenen Beitrag für eine regionale Verteidigungsorganisation zu bestimmen, betonte aber zugleich das Fortdauern der militärischen Verantwortung Großbritanniens. ${ }^{99}$ NSC 129/1 und die begleitende Staff Study hatten aber, wie bereits am Rande der Diskussion über die USPolitik gegenüber Mosaddeg erwähnt, die Schwerpunktsetzung der US-Strategie weg von kollektiven Verteidigungsanstrengungen und hin zu pro-westlich orientierter Stabilisierung der jungen Nationen festgeschrieben und zu diesem Zweck den ,fullest practicable use of psychological and political programs (including special political measures) and of economic and military aid programs and technical cooperation" empfohlen. ${ }^{100}$ Dies war aber nur teilweise vereinbar mit der Hoffnung der konservativen Regierung in London, mittels MEDO eine langfristige Zusage Kairos zur Nutzung der Militärbasis am Suezkanal zu erlangen. Nachdem die neue nationalistische Regierung im Oktober 1952 ihre Ablehnung signalisiert hatte, endeten die Bemühungen um den Aufbau einer auf Ägypten fokussierten regionalen Verteidigungsorganisation. ${ }^{101}$

Der Druck auf die USA, sich nachdrücklicher an den Verteidigungsplanungen der Nahostregion zu beteiligen, nahm weiter zu. Die militärischen Stellen selbst hatten dem ungewollt Vorschub geleistet, da sie ihre britischen Verbündeten immer wieder drängten, die Notfallplanungen auf

\footnotetext{
${ }^{98}$ Zit. aus RuAnE, Anglo-American Relations, S. 5; bereits im November 1951 stellte Churchill fest, es sei „[...] of utmost importance to get the Americans in."; zit. aus BDEE A, 3, I, S. $111 n 2$.

${ }_{99}$ NSC, NSC 129/1, 24.4.52, FRUS 52-54, IX, S. 223f.; vgl. den frühen Entwurf der Studie in NSC Draft Study, 27.12.51, FRUS 51, V, S. 257-64; die JCS wehrten sich vergeblich gegen die Erwähnung von „,token forces“ und kritisierten die anvisierte Entsendung als ,militarily inadvisable“. Zu den JCS-Empfehlungen die Türkei betreffend siehe oben Anm. 95; vgl. POOLE, History of the JCS: $I V$, S. 178.

${ }^{100}$ NSC 129/1, 24.4.52, FRUS 52-54, IX, hier S. 224; „special political measures“ sind ein Euphemismus für geheimdienstliche Operationen. Zum Iran-Bezug vgl. oben S. 109f.; dementsprechend bewertete die Staff Study den Wert des MEC weniger in der Abwehr externer Aggression: ,,its greatest usefulness may actually lie in the opportunity that it provides for strengthening local military forces and their ties with the West [...]"; NSC Staff Study, 24.4.52, ,Near East (4)‘, WHO, NSCS, Disaster File Ser., Box 64, DDEL, S. 18f.

${ }^{101}$ Vgl. Eden Cabinet Memos, 27.10.8.52; 16.2.53, BDEE A, 3, I, S. 118ff., $125 f$.
} 
die Verteidigung des sogenannten „Äußeren Ringes“ zu fokussieren, also entlang des TaurusGebirges im Süden und Südosten der Türkei und des Zāgros-Gebirges quer durch Iran bis hin zur Straße von Hormus. Angesichts amerikanischer Zurückhaltung und der eigenen begrenzten Kapazitäten plädierten die Briten dagegen auf die Konzentration auf einen „Inneren Ring“ die Sicherung des britischen Stützpunktes in Ägypten mittels Verteidigung einer Linie entlang des Jordans, weiter nach Norden entlang des Antilibanon-Gebirges bis zum Taurusgebirge nahe Malatya in der Türkei, somit in der Theorie die Ölquellen am Golf, Iran, Irak und den Großteil des türkischen Territoriums preisgebend. ${ }^{102}$ Eine gemeinsame anglo-amerikanische Studie hatte noch im April 1951 angesichts fehlender militärischer Kapazitäten ein weiteres Mal den „Inneren Ring“ favorisiert. ${ }^{103}$ Der Policy Planning Staff im State Department kritisierte im Mai 1952 die bestehenden militärischen Defizite im Nahen Osten heftig. Diese kämen einer Einladung an Moskau ,[...] to a shift in the theater of primary pressure“ gleich, zumal Großbritannien wohl nicht einmal in der Lage wäre, den „Inneren Ring“ zu verteidigen. ${ }^{104}$ Wenige Monate später bereits forderte das State Department eine Studie über die notwendigen Kapazitäten und Kosten für eine ,forward defense“ in der Nahostregion an. Das DOD berichtete daraufhin im November, dass gegenwärtig nur 12 von 19 für die angemessene Verteidigung des „Äußeren Ringes“ notwendigen Divisionen zur Verfügung stünden. ${ }^{105}$

Mit dem Amtsantritt der Eisenhower-Administration verlagerte sich die militärische Strategie im Nahen und Mittleren Osten alsbald auf die Northern Tier-Staaten. John Foster Dulles gab im Mai 1953 im Anschluss an eine ausgedehnte Reise durch die Region das MEDO-Konzept endgültig auf und sprach sich nun für die Konzentration auf die nahöstlichen und südasiatischen Staaten entlang der sowjetischen Südgrenze aus - eine NSC-Studie (NSC 155/1) vom Juli 1953 nannte derweil explizit die Türkei, Irak, Syrien, Iran und Pakistan als Schwerpunkt der neuen Regionalstrategie. ${ }^{106}$ Zugleich implizierte die neue Nahostpolitik eine bewusste Distanzierung

\footnotetext{
${ }^{102}$ Die Briten favorisierten lange eine noch weiter zurückgezogene Verteidigungslinie zwischen israelischer Küste und dem Jordan-Fluss, ungefähr in Höhe Ramallahs oder, alternativ, weiter nördlich im Libanon-Gebirge. Vgl. CoHEN, Fighting World War Three, S. 166ff.; Vgl. Agreed US-UK Memo of Discussions, encl. to Jessup Memo, 25.7.50, FRUS 50, V, S. 188-191; COS Committee Memo, 15.9.50, BDEE B, 4, II, S. 77-100; vgl. COHEN, Fighting World War Three, S. 163-7; die JCS kritisierten: „Defense of the Inner Ring does not constitute a defense of the Middle East, but defense of Egypt.“; Draft of Comments, 19.10.50, BDEE B, 4, II, S. 119.

${ }^{103}$ Vgl. CINCNELM and Cs-in-C, Middle East Memo, 13.3.51, FRUS 51, V, S. 94f.; siehe auch ebd., S. 28 n5; vgl. COS Committee Memo, 18.12.51, BDEE B, 4, II, S. 290-305; PoOLE, History of the JCS: IV, S. 189-92; CoHen, Fighting World War Three, S. 274ff.; DeVEREuX, Formulation, S. 105ff.; Ross, American War Plans, S. $146 f$.

${ }^{104}$ Policy Planning Staff Memo, 21.5.52, FRUS 52-54, IX, S. 232ff., hier 233; hier auch ein expliziter Verweis auf das für spätere Planungen entscheidende Element einer Sperrung der Bergpässe durch den Einsatz von Atomwaffen.

${ }^{105}$ Vgl. Matthews Letter, 15.8.52, ebd., S. 266f.; vgl. ebd., S. 267 n3; State-JCS Meeting Minutes, 29.10.52; StateJCS Meeting Minutes, 28.11.52, ebd., S. 305-10, 319-26; vgl. PoOLE, History of the JCS: IV, S. 191ff.; WATSON, History of the JCS: V, S. 325; hier auch Hinweise auf die gegensätzliche Position der Navy. Zu den begleitenden anglo-amerikanischen Gesprächen über den Strategiewandel vgl. CoHEN, Fighting World War Three, S. 273ff., 282f.; zum Wandel der britischen Strategie vgl. DEVEREUX, Formulation, S.100-20.

106 Vgl. 147th NSC Meeting, 1.6.53, FRUS 52-54, IX, S. 379-86, insbesondere die angefügten NSCGrundsatzentscheide; NSC, NSC 155/1, 14.7.53, ebd., S. 399-406; NSC 155/1 sprach nun vom ,need to act in concert with the United Kingdom to the greatest extent practicable“", betonte aber zugleich das Recht ,,to act [...] alone". Die Studie empfahl geheime und informelle Gespräche über die regionale Verteidigung und gezielte Militärhilfe an bevorzugte Staaten - eine regionale Organisation zu diesem Zweck wurde gegenwärtig als nicht realistisch eingeschätzt. Das mittelfristige Ziel einer regionalen Organisation der Northern Tier-Staaten wurde
} 
von London. Deputy Assistant Secretary of State Jernegan vermerkte im Juni 1953 explizit, dass eine Fortsetzung der bisherigen Haltung „to follow the British lead“ zwangsläufig die Zerstörung von ,[...] all of our influence in the Arab world and Iran and most of it in South Asia“ zur Folge haben würde. ${ }^{107}$ Erleichtert wurde die neue US-Haltung auch durch eine Annäherung zwischen den Positionen von State und Defense Department. Eine JCS-Studie vom Oktober 1953 hielt nun mittelfristig die Verteidigung des sogenannten „Äußeren Ringes“ entlang des iranischen Zāgros-Gebirges für realisierbar, dabei eine Kooperation der Northern Tier-Staaten zu diesem Zweck für günstig befindend. ${ }^{108}$ In der nun folgenden Phase reagierte die Eisenhower-Administration reaktiv, aber immer mit Wohlwollen, auf Initiativen der regionalen Staaten, nicht zuletzt um eine Wiederholung des im Kontext der MEC und MEDOPlanungen entstandenen Eindrucks westlicher Fremdbestimmung zu vermeiden. Ermuntert von amerikanischen Zusagen künftiger Militärhilfe schlossen die Türkei und Pakistan im April 1954 einen Vertrag über freundschaftliche Zusammenarbeit, der auch Kooperation auf dem Feld der Verteidigung einschloss und explizit den Beitritt weiterer Staaten anvisierte. ${ }^{109}$

Warnende Stimmen aus der Regierungsbürokratie ignorierend, bewegte sich die EisenhowerAdministration entschlossen auf eine Umsetzung des Northern Tier-Konzepts zu. ${ }^{110}$ Eine Aktualisierung der nur ein Jahr alten Regionalstrategie - der NSC definierte die Iranpolitik weiterhin in separaten Studien - im Juli 1954 (NSC 5428) identifizierte den turkopakistanischen Pakt als „best prospect“ für den Aufbau eines indigenen regionalen Verteidigungsarrangements und empfahl die Ermutigung einer solchen Organisation seitens der USA sowie die Konzentrierung amerikanischer Militärhilfe auf die zukünftigen Mitgliedsstaaten. In diesem Zusammenhang ist es wichtig festzustellen, dass man in Washington in rein militärischer Hinsicht nur einen begrenzt positiven Effekt vom Aufbau einer Verteidigungsorganisation erwartete und die Vorteile zuvorderst auf der politischen und psychologischen Ebene sah: „Such a grouping should strengthen Western-oriented elements and tend to bring about greater awareness of the Soviet threat and greater willingness to

auch in die Basic National Security Policy aufgenommen. Siehe Lay Report, NSC 162/2, 30.10.53, FRUS 52-54, II, S. 592f.; vgl. WATSON, History of the JCS: V, S. 331f.

107 Jernegan Memo, 17.6.53, FRUS 52-54, VI, S. $992 \mathrm{f}$.

${ }^{108}$ Tatsächlich war es eine verkürzte,,äussere“ Linie, dieses Mal nur bis etwa Basra und nicht bis Hormus reichend. Obgleich die militärischen Kapazitäten weiterhin als unzureichend angesehen wurden, erklärten sich die JCS im Juni 1954 bereit, militärische Planungen auf Grundlage der Zāgros-Linie zu beginnen. Vgl. WATSON, History of the JCS: V, S. 336-40; JCS History Iran, S. 44ff.; Jernegan Letter, 9.11.53; JCS Memo, 14.11.53, FRUS 52-54, IX, S. 424-8, 430ff.

${ }_{109}$ Der NSC hatte bereits im März Militärhilfe an Pakistan beschlossen. Vgl. PERSSON, Baghdad Pact, S. 125-8; WATSON, History of the JCS: V, S. 343ff.; vgl. State \#483, 29.12.53; ed. note, FRUS 52-54, XI, S. 1837f., 1845f.; State \#686, 24.12.53, FRUS 52-54, IX, S. 439ff.; JCS Memo, 10.12.53, Memo to Chairman Planning Board, o.D. [24.8.53], ,091.370 Iran', Project Decimal Files, 1953; Office of Military Assistance, OASD/ISA, Box 52, RG 330, NACP; zu den Verhandlungen vgl. PODEH, Quest for Hegemony, S. 64-76.

${ }^{110}$ Wie so häufig demonstrierte wieder einmal das OIR im State Department besondere Weitsicht und warnte vor den Problemen und Konsequenzen einer US-gesponserten regionalen Verteidigungsorganisation, insbesondere der Auswirkungen einer solchen auf die strukturbildenden anglo-ägyptischen, anglo-iranischen und arabischisraelischen Konflikte. Siehe OIR Paper, 3.5.54, FRUS 52-54, IX, S. 503f. 
cooperate both regionally and with the West". ${ }^{111}$ Hier zeigt sich ein weiteres Mal die prinzipielle Kontinuität in der allgemeinen Nahostpolitik zwischen Truman- und EisenhowerAdministration. Erstere hatte, wie bereits oben analysiert, im April 1952 mit NSC 129/1 eine ähnliche Strategie gerichtet auf die bestehenden und zukünftigen Führungsschichten der jungen nahöstlichen Staaten im Sinne einer permanenten Interventionspolitik formuliert. ${ }^{112}$ NSC 5428 betonte die Unabhängigkeit der amerikanischen Nahostpolitik vom früheren britischen Hegemon, aber auch von Israel, und insbesondere die „indigenous nature“ der angedachten Verteidigungsorganisation, also vorerst ohne formelle Anbindung an westliche Staaten und damit auch unabhängig von den imperialen Interessen Londons. ${ }^{113}$

Ermuntert von amerikanischen Zusagen für die Lieferung großzügiger Militärhilfe schloss als nächstes der Irak im Februar 1955 einen Beistandspakt mit der Türkei ab. ${ }^{114} \mathrm{Zu}$ diesem Zeitpunkt unterstützten die USA das entstehende Bündnis als zentralen Baustein in der Konzeption einer Northern Tier-Organisation mit Nachdruck, signalisierten aber zugleich Zurückhaltung, was einen möglichen eigenen Beitritt zum Pakt anging. ${ }^{115}$ Entscheidend für den weiteren Fortgang der Ereignisse war die Kehrtwende in der britischen Politik gegenüber dem Northern Tier. Anfangs dominierte in London die Besorgnis, die neue amerikanische Politik seit 1953 ziele auf eine Verdrängung der verbliebenen Reste der britischen

111 NSC, NSC 5428, 23.7.54, ebd., S. 527; vgl. ebd., S. 530f.; ähnlich die Begründung für die Erhöhung der Militärhilfe an ausgesuchte Staaten mit dem Ziel, ,[...] to induce internal stability and political orientation towards the West"; ebd.; vgl. CIA; NIE 30-54, 22.6.54, ebd., S. 516-20.

112 Vgl. oben S. 109f; NSC 5428 implizierte eine noch stärkere Ausrichtung auf die militärischen Eliten, wenngleich offenbar eine Intervention des State Department zu einer stärkeren Betonung wirtschaftlicher und sozialer Faktoren führte. Siehe FRUS 52-54, IX, S. 526 n2; die konkreten Maßnahmen der Studie sahen explizit den "support“ von ,leadership groups which offer the best prospect of orderly progress towards free world objectives“ und eine pro-westliche Beeinflussung der Ideenwelt und politischen Kulturen vor (,,Seek to increase the participation of intellectuals in Western-oriented activities."); ebd., S. 529; die in der FRUS-Version noch klassifizierte Maßnahme $N^{\circ} 1$ findet sich in einer 1996 freigegebenen Version des Dokuments. Sie lautet lapidar: „Conduct covert activities in support of U.S. objectives.“; NSC 5428, Überarbeitung vom 2.11.55, DDRS: CK2349102499; zur Problematik der ,leadership groups“vgl. auch Guy LARON, Origins, S. $76 f$.

113 NSC 5428, FRUS 52-54, IX, hier S. 527; allerdings war man sich weiterhin der Vorteile einer fortgesetzten britischen Präsenz bewusst, wie eine begleitende Staff Study erneut betonte: ,Therefore, although the trend is for United States influence to replace British influence in the area as a whole, British ability to aid in the preservation of Western security interests in the area should not be minimized."; NSC Staff Study on NSC 5428, o.D. [Juli 1954], DDRS: CK2349102499; vgl. WATSON, History of the JCS: V, S. 346; die Debatte über die Studie im NSC wurde allerdings kontrovers geführt. Hauptstreitpunkt war der Einsatz militärischer Mittel im Falle einer weiteren kriegerischen Eskalation des arabisch-israelischen Konflikts. Die verabschiedete Annex zu NSC 5428 sah als ultima ratio den Einsatz einer militärischen Blockade bzw. weiterer militärischer Maßnahmen gegen den Aggressor vor, also auch theoretisch gegen Israel. Diese Passagen fehlen ebenfalls in der FRUS-Version. Vgl. Discussion at the 207th Meeting of the NSC, 23.7.54, DDRS: CK2349132883; vgl. CONDIT, History of the JCS: $V I$, S. $169-74$.

${ }^{114}$ Irak wurde durch einen Notentausch im April 1954 zum berechtigten Empfänger von Waffenlieferungen im Rahmen des Mutual Defense Assistance Program (MDAP). Zum MDAP vgl. WATSON, History of the JCS: V, S. 201-9; vgl. REID, Baghdad Pact, S. 170-4; die eigenständigen Rollen der Türkei und des Irak beim Zustandekommen des Pakts betont SANJIAN, Formulation.

${ }^{115}$ Vgl. State \#463, 15.2.55, FRUS 55-57, XII, S. 19f.; die USA betonten die bestehende Unterstützung für diese Staaten mit Verweis auf die militärischen Verpflichtungen gegenüber der Türkei und Pakistan im Rahmen der NATO bzw. der im September 1954 vereinbarten Southeast Asia Treaty Organization (SEATO) sowie auf die USMilitärhilfe an Irak und Iran. Zugleich betonte man zu diesem Zeitpunkt, „no objection“ hinsichtlich eines britischen Beitritts zu haben. Zu den Verhandlungen vgl. Persson, Baghdad" Pact, S. 138-54, 204-10; PODEH, Quest for Hegemony, S. 91-125; London hoffte zu diesem Zeitpunkt, die gemeinsame anglo-amerikanische Politik $\mathrm{zu}$ wahren, war aber negativ eingestellt gegenüber dem Bemühen der amerikanischen Berater in Iran, den Schah zu einem Beitritt zu bewegen. Vgl. H.A.F. Hohler Minute, 14.2.55, FO 371/114820; vgl. Makins to FO, No. 63, 28.1.55, FO 371/115469, TNA:PRO; Botschaft Teheran an AA, 26.1.55, B 12/1008, PAAA. 
Vormachtstellung in der Region und insbesondere im Irak $^{116}$ und im Anschluss auf eine räumliche Umorientierung der Militärstrategie hin zum östlichen Teil der Nahostregion angesichts der (vorläufigen) Lösung des Anglo-Ägyptischen Konflikts im Oktober 1954 inklusive der zugesagten Räumung der Suez-Basis. Nun erkannte man aber auf britischer Seite die Vorteile des Konzepts für die Aufrechterhaltung der eigenen Position. ${ }^{117}$ Ähnlich wie während der Bemühungen um die Verwirklichung der MEC und MEDO-Konzeptionen in den Jahren zuvor, hoffte man in London wieder einmal auf eine Bestätigung imperialer Privilegien im Kontext einer vorgeblich gleichrangigen Verteidigungsanstrengung - im Fokus stand nun die Erneuerung des 1957 auslaufenden Anglo-Irakischen Vertrags von 1930. ${ }^{118}$ Bewusst eine erneute Konfrontation mit dem ägyptischen Nationalismus und Nasser in Kauf nehmend, entschloss sich das Vereinigte Königreich im April 1955 zugunsten des Beitritts zum türkischirakischen Bündnis, nunmehr als Bagdad-Pakt bezeichnet. ${ }^{119}$

Was auf den ersten Blick wie eine erfolgreiche Implementierung der in den beiden vorangegangenen Jahren seitens der Eisenhower-Administration propagierten Ideallösung für die Frage der Verteidigung entlang der NATO-Südflanke erscheinen musste, stieß aber auf unerwartete Zurückhaltung in Washington, eine neuerliche Vertrauenskrise in der angloamerikanischen special relationship auslösend. Der Tausch der bisherigen Rollen bezogen auf die Idee einer Northern Tier-Organisation zwischen Washington und London hatte mehrere Gründe. Die Kehrtwende in der amerikanischen Nahostpolitik erklärt sich in erster Linie aus der unerwartet scharfen ägyptischen Reaktion auf die Entstehung des Bagdadpaktes - Nasser betrachtete die Aufwertung Iraks als Herausforderung der Führungsrolle Kairos in der arabischen Welt und reagierte postwendend mit einer scharfen Propagandakampagne gegen das Projekt mit dem Ziel, den Beitritt weiterer arabischer Staaten zu vereiteln. Zugleich organisierte Ägypten eine konkurrierende Allianz gemeinsam mit Syrien und Saudi-Arabien. ${ }^{120}$

116 Vgl. Shuckburgh Minute, 11.1.55, BDEE B, 4, III, S. 353f.; um solche Bedenken zu zerstreuen, stimmte Washington in einem ungewöhnlichen Schritt im Februar 1954 einer Vereinbarung zu, die explizit die Koordination der vereinbarten Militärhilfe mit dem britischen Partner und die prinzipielle Anerkennung des britischen Militärmonopols im Irak zusagte. Vgl. Memo of Understanding, 26.2.54, FRUS 52-54, IX, S. 2371ff.; Spannungen vor Ort existierten jedoch weiterhin: „No Englishman living in Iraq can remain unmoved as he sees a horde of highly paid American experts sweeping into a country whose traditional ties are with ourselves and hears them denigrating all that Britain has done here in the past and is attempting to do at the present."; Botschafter Sir John Troutbeck im Dezember 1954, zitiert aus Sommerville Memo, 26.5.55, FO 371/115585, TNA:PRO; Hintergründe in RuANE, Anglo-American Relations, S. 8f.; PERSSON, Baghdad Pact, S. $141 \mathrm{ff}$.

${ }_{117}$ Der Anglo- Ägyptische Vertrag sah die (militärische) Räumung der Suez-Basis innerhalb von 20 Monaten im Gegenzug für ein siebenjähriges Recht auf Reaktivierung der Basis im militärischen Ernstfall vor. Vgl. DEVEREUX, Formulation, S. 129-41; OvendAle, Transfer of Power, S. 74-8; LuCAS, Divided We Stand, S. 18-39; Falla Memo, 1.10.54, Anglo-Egyptian Agreement Regarding the Suez Canal Base, 19.10.54, BDEE B, 4, III, S. 313ff., 320-4; „The Canal Zone base could play no useful part at the start of a global war in support of operations in the Baghdad Pact area on account of the remoteness both of its geographical position and its chances of survival in nuclear warfare."; Chiefs of Staff Report, 3.7.56, BDEE A, 3, I, S. 154.

${ }^{118}$ Siehe PERSSON, Baghdad Pact, S. 229 n52; gleichzeitig wollte man die Fehler von 1948 mit dem Vertrag von Portsmouth dieses Mal vermeiden und war daher bemüht ,[...] not to offend Iraq nationalist sentiment"; Eden Memo, C.(55) 70, 14.3.55, fos 148-152, PREM 11/1408, TNA:PRO.; vgl. JASSE, Baghdad Pact.

119 Das türkisch-irakische Abkommen hatte im Artikel VI die Einrichtung eines permanenten Ministerrats vorgesehen für den Fall des Beitritts mindestens zweier weiterer Mächte. Siehe Eden Cabinet Memo, 14.3.55, $B D E E$ B, 4, III, S. 397-401; C.C.(55) 24th Conclusions, 15.3.55, CAB 128/28, TNA:PRO, fol. 215-216; COHEN, Defending the Northern Tier, S. $84 \mathrm{ff}$.

120 Vgl. Cairo \#1312, 8.3.55, FRUS 55-57, XII, S. 29-32; zu Nassers Reaktion und zur syrisch-saudischägyptischen Allianz vgl. PoDEH, Quest for Hegemony, S. 103-17, 126-38; grundlegend für den Tausch der 
Diese Polarisierung der Region stellte die grundsätzliche Stabilität der „rear area“ hinter dem nördlichen Riegel in Frage und gefährdete damit die übergeordneten strategischen Ziele der Eisenhower-Administration. Ein drohender Konflikt mit Nassers Ägypten eröffnete zugleich der Sowjetunion die Gelegenheit, so die berechtigte Befürchtung, die westlich inspirierte Verteidigungsstruktur im Nahen und Mittleren Osten durch eine Annäherung an die revolutionäre Führung in Kairo zu ,überspringen“ (,leapfrogging“) eine Entwicklung, die dann im September mit dem ägyptischen Waffengeschäft mit dem Ostblock in der Tat Realität werden sollte. Die Kontroverse um den Pakt unterminierte zudem die parallel laufende Operation ALPHA, eine ambitionierte anglo-amerikanische Initiative gerichtet auf eine umfängliche und abschließende Lösung des arabisch-israelischen Konflikts inklusive der offenen Grenzfragen, der Anerkennung Israels und einer Regelung der Flüchtlingsproblematik. ${ }^{121}$ Eine Regulierung des Nahostkonflikts war ohnehin aus Sicht der Eisenhower-Administration die Voraussetzung für eine sinnvolle Verteidigungsanstrengung der Region als Ganzes, da die arabischen Staaten Israel als hauptsächliche externe Bedrohung wahrnahmen. Zudem war eine amerikanische Beteiligung am Bagdadpakt darüber hinaus aus innenpolitischer Perspektive nicht opportun. Die pro-israelische Lobby in der amerikanischen Innenpolitik, so die Überzeugung innerhalb der Administration, würde zum Ausgleich unmittelbar auf eine Sicherheitsgarantie für den jüdischen Staat und auch auf Waffenlieferungen drängen, was den eigentlichen Zweck des Paktes, die pro-westliche Orientierung der regionalen Führungen, erneut in Frage stellen würde. ${ }^{122}$

Die Eisenhower-Administration drängte daher - entgegen der vorherrschenden Meinungen auf Seiten des Defense Department, des State Department, der JCS und der CIA - auf Zurückhaltung, um eine weitere Polarisierung in der Region zu vermeiden, und unterstützte vorerst nur den Beitritt der nicht-arabischen Staaten Pakistan und Iran zum Paktsystem. ${ }^{123}$ Beide Staaten traten dem Paktsystem schließlich im September und November 1955 bei. Während der pakistanische Beitritt innerhalb der Administration auf große Zustimmung stieß, war die Beteiligung Irans weiterhin umstritten. Wie oben ausgeführt, hatte NSC 5402 vom Januar 1954 eine solche iranische Beteiligung und ein damit einhergehendes

anfänglichen britischen und amerikanischen Positionen ASHTON, Hijacking; zu den Hintergründen vgl. RuANE, Collective Defense.

${ }^{121}$ Vgl. State \#1643, 31.3.55, FRUS 55-57, XII, S. 46ff.; Ward Minute, 2.2.55, BDEE A, 3, I, S. 362; aus der umfangreichen Literatur zu ALPHA vgl. PERSSON, Baghdad Pact, S. 186-224; FREIBERGER, Dawn over Suez, S. 107-32; TAKEYH, Eisenhower Doctrine, S. 48-72, HAHN, Caught, S. 182-93.

${ }^{122}$ Vgl. ebd., S. $189 f$.; Under Secretary of State Herbert Hoover, Jr., verwies explizit auf die Israel-Lobby und warnte den Präsidenten, ein US-Beitritt, ,[...] might even fail of ratification by the Senate, through pressure generated in New York and other big metropolitan areas of the US.“; MemCon, 11.7.55, ,July 1955 (2)“, WHO, OSANSA, Special Assistant Ser., Chronological SubSer., Box 1, DDEL.

${ }^{123}$ Den US-Beitritt im Grundsatz befürwortend State-Defense Working Group Memo, 6.6.55, FRUS 55-57, XII, S. 63-70; ähnlich JCS Memo, 16.6.55, ebd., S. 70-4; zustimmend wenngleich weitaus skeptischer CIA, NIE 3055, 21.6.55, ebd., S. 77-97; die CIA sah zudem die Chancen einer iranischen Beteiligung als eher unwahrscheinlich an. Siehe ebd., S. 84; Ende Juni 1955 stand bereits der Entscheid des (vorläufigen) Nichtbeitritts und der Einnahme einer Position der ,close collaboration but non-adherence“. Siehe MemCon State-DOD, 30.6.55; State Position Paper, 11.7.55, ebd., S. 127ff., 129-32; zum Zitat vgl. die FRUS-Editoren in ebd., S. 684 n2; es entstammt Mohammad Rezā Memo, o.D., encl. to Eisenhower Memo, 14.12.54, ,231st Meeting', Official Meeting Minutes, Box 13, RG 273, NACP; zur britischen Unterstützung des pakistanischen und iranischen Beitritts vgl. Arthur, Brevis, Fry, Bromley Minutes, 23.-25.4.55, BDEE B, 4, III, S. 413ff. 
Aufrüstungsprogramm ins Auge gefasst, wenngleich es intern weiterhin Zweifel an der Zweckmäßigkeit dieser Maßnahmen gab. In den internen Beratungen über eine neue NSCStudie zu Iran im Anschluss an die formelle Beendigung der Ölkrise waren Umfang und Zielsetzung der US-Militärhilfe für Iran und die eventuelle Beteiligung des Landes am entstehenden Paktsystem die hauptsächlichen Streitpunkte. ${ }^{124}$ Die erklärte Bereitschaft der iranischen Führung, die traditionelle Neutralität Irans aufzugeben und sich den ,leading nations of the free world“ anzuschließen, wurde vom State Department als Argument benutzt, die bisherige Zurückhaltung aufzugeben. ${ }^{125}$ NSC 5504, am 13. Januar 1955 verabschiedet, erklärte es zum Ziel der US-Politik, die iranischen Streitkräfte auszubauen, damit diese im Falle eines sowjetischen Angriffs ,[...] make a useful contribution to Middle East defense“. Zugleich stellte die Studie fest, dass dies ein „long-term program involving U.S. expenditures substantially in excess of present levels“ impliziere. ${ }^{126}$ Des Weiteren sah NSC 5504 eine Fortsetzung der amerikanischen Wirtschafts- und Finanzhilfen bis zum Jahr 1957 vor, in dem ein Anstieg der Öleinkünfte auf über \$200 Millionen erwartet wurde, was Iran in der Lage versetzen würde, endlich auf eigenen Füßen zu stehen.

Im Kern ging es aber weniger um die - zu diesem Zeitpunkt recht unrealistisch erscheinende Aufwertung iranischer Militärmacht auf ein Niveau, welches einen tatsächlichen Mehrwert für die Verteidigung der Nahostregion bedeutet hätte. NSC 5504 begründete denn auch die Aufrüstungspläne eher psychologisch denn rein militärisch. Es gelte, die gegenwärtige prowestliche Ausrichtung iranischer Politik unter dem Schah zu festigen durch die Schaffung eines „sense of security and participation in the free world“ mit dem Ziel ,[...] to change Iran from a liability to a positive anti-Communist asset in Asia“. ${ }^{127}$ Geheime Stabsgespräche zwischen britischen, amerikanischen und türkischen Planern hatten bereits im Januar und Februar 1955 die bestehenden Kriegspläne für die nahöstliche Verteidigung aktualisiert. Statt 19 Divisionen wie noch im Herbst 1952 sah man 7 1/2 Divisionen als ausreichend an, da nun der Einsatz von Nuklearwaffen von Beginn an einkalkuliert wurde. Atomschläge gegen die sowjetischen Truppenverbände bzw. ihre Kommunikationslinien waren dabei die entscheidende Komponente, während ein möglicher konventioneller Beitrag der iranischen Streitkräfte an Bedeutung verlor. Andererseits sahen die Pläne Kriegshandlungen vor allem auf iranischem Territorium entlang der Zāgros-Linie vor, was eine Zusammenarbeit mit Iran wiederum unumgänglich machte. ${ }^{128}$ In den darauffolgenden anglo-amerikanischen politisch-

\footnotetext{
${ }^{124}$ Vgl. Bowie Memo, 11.1.55, FRUS 55-57, XII, S. 683.

${ }^{125} \mathrm{Zit}$. aus Jernegan Memo, 11.1.55, ebd., S. 684; vgl. Tehran \#1016, 3.11.54, FRUS 52-54, IX, S. 556f.; zur verbreiteten Skepsis unter iranischen Offiziellen bez. einer Aufgabe der iranischen Neutralität und den innenpolitischen Folgen eines solchen Schritts vgl. Tehran \#1341, 17.12.53, FRUS 52-54, X, S. 850ff.

${ }^{126}$ NSC, NSC 5504, 15.1.55, FRUS 55-57, XII, S. 693f.

${ }^{127}$ Ebd., S. 693; das bisherige Programm sah ein Gesamtprogramm an Militärhilfe von 1950 bis 1957 von \$124 Millionen vor - im Anschluss sollten \$10 Millionen jährlich zur Aufrechterhaltung der erreichten Kampfkraft ausreichen. Vgl. Jernegan Memo, 7.1.55, FRUS 55-57, XII, S. 676-80; Memo ,Comment on NSC 5402/1', 10.1.55, ,Iran (3)‘, WHO, NSCS, Special Staff File Ser., Box 4, DDEL.

${ }_{128}$ Vgl. CONDIT, History of the JCS: VI, S. 153f.; COHEN, Defending the Northern Tier, S. 108-15; JCS History Iran, S. 47; die britischen Militärs schätzten die iranische Kampfkraft offenbar als weitaus geringer ein als ihre amerikanischen Kollegen. Vgl. Combined Turkey-UK-US Middle East Defence Study, 4.3.55; COS Committee
} 
militärischen Gesprächen über die Nahoststrategie äußerten dann allerdings die Briten Bedenken über mögliche negative Folgen eines iranischen Beitritts zum Pakt. ${ }^{129}$

Mohammad Rezā selbst steuerte bereits ab April 1955 vehement auf eine Aufgabe der iranischen Neutralität und einen Beitritt zum Bagdadpakt zu, offenkundig in der Erwartung einer amerikanischen Belohnung in der Form zusätzlicher und generöser Militärhilfe. ${ }^{130}$ Während auf US-Seite die Zweifel bezüglich der Folgen einer Aufgabe der traditionellen iranischen Neutralität wuchsen, fürchtete der Schah offenkundig diese einmalige Gelegenheit (,missing the boat“) zum Beitritt zu einer regionalen Verteidigungsorganisation zu verpassen. ${ }^{131}$ Im September 1955 übermittelte Mohammad Rezā dem neuen US-Botschafter Selden Chapin sodann eine Forderungsliste im Gegenzug für einen iranischen Beitritt: Zusagen über Wirtschafts- und Finanzhilfe, eine militärische Beistandsgarantie seitens Großbritannien und der USA und zusätzlich die Anerkennung der iranischen territorialen Ansprüche auf Bahrain, zu dieser Zeit noch unter informeller britischer Kontrolle. ${ }^{132}$ Secretary of State John Foster Dulles ließ die iranische Führung warnen, dass die USA keineswegs die Absicht hätten, den iranischen Beitritt „zu kaufen“. ${ }^{133}$ Tatsächlich schwand die Begeisterung für eine iranische Beteiligung in Washington immer mehr. Intern leisteten die JCS weiterhin Widerstand gegen die Forderungen nach einem erweiterten Militärhilfeprogramm für Iran seitens des State Department, der CIA, und des Country Team in der Teheraner Botschaft. ${ }^{134}$ Im Anschluss an das ägyptische Waffengeschäft mit dem Ostblock im September 1955 verschob sich die

Memo, 23.3.55, BDEE B, 4, III, S. 374-90, 404-10; die Briten gingen davon aus, dass „,both sides would use nuclear weapons from the outset [...]“; MemCon, 23.6.55, FRUS 55-57, XII, S. 106.

${ }^{129}$ Vgl. ebd.; die Amerikaner widersprachen zu diesem Zeitpunkt heftig. Iran sei ,on the verge of a rebirth“, sowohl ökonomisch wie auch militärisch. London sah zwar die politischen Vorteile eines iranischen Beitritts, betonte aber dessen mangelnde Stabilität und Widerstandsfähigkeit. Die britische Seite ,[...] did not expect to enter into a military commitment to Iran and added that one could not expect the Iranians to bring any appreciable military capability to the Pact."; zit. aus MemCon, 11.8.55, ebd., S. 140; zur Bedeutung Irans aus US-Sicht vgl. ebd., S. 143, 151; vgl. COS Memo, 25.8.55, BDEE B, 4, III, S. 426-30; die interne britische Position zu einem iranischen Beitritt war , [...] while continuing to favour Persian adherence, not to press for it or to pay any high price for it" und zudem " ein "direct military commitment to Persia“ auszuschließen. Shuckburgh Memo, 10.5.55, FO 371/115511, TNA:PRO; zum Hintergrund der anglo-amerikanischen Gespräche vgl. Eveland, Jr. Memo, 19.5.55, ,OCB 091.4 Near East (File \#2) (2) ', WHO, NSCS, OCB Central File Ser., Box 78, DDEL; Brief for Chief of the Air Staff, 18.10.55, AIR 8/2043, TNA:PRO; COHEN, Defending the Northern Tier, S. $115 \mathrm{ff}$.

${ }_{130}$ Vgl. Baxter Memo, 4.2.55, FRUS 55-57, XII, S. 703-8; George V. Allen, der frühere US-Botschafter in Teheran war von Januar 1955 bis Juli 1956 als Nachfolger von Henry Byroade Assistant Secretary of State for Near Eastern, South Asian, and African Affairs. Siehe auch Tehran \#2062, 11.4.55, ebd., S. 729ff.; OIR Report Abstract, 1.9.55, in: ALEXANDER/NANES, United States and Iran, S. $275 \mathrm{f}$.

${ }_{131}$ MemCon, 11.7.55, ,July 1955 (2)‘, WHO, OSANSA, Special Assistant Ser., Chronological Subs., Box 1, DDEL.

${ }^{132}$ Siehe Tehran \#386, 3.9.55, FRUS 55-57, XII, S. 765f.; Bahrain war zwischen 1602 und 1783 unter iranischer Kontrolle gewesen. Chapin besetzte im Juli den seit Januar vakanten Botschafterposten. Die Berufung des von Eisenhower favorisierten Julius Holmes war im US-Senat gescheitert. Der Rückzug der Berufung von Holmes wurde letztlich mit gesundheitlichen Problemen begründet. Holmes wurde schließlich US-Botschafter in Iran unter der Kennedy-Administration sechs Jahre später. Vgl. Henderson Memo, 30.3.55, ,LW Henderson - Chron, JanMar 1955', Lot 67 D 44, Records of Loy Henderson, Box 1; siehe unten, Kapitel IV.

${ }^{133}$ State \#460, 17.9.55, FRUS 55-57, XII, S. 773ff.; Hoover Jr. befand die Forderungen des Schahs offenbar als ,verging on the absurd"; Washington Embassy to FO, No. 2111, 7.9.55, FO 371/115519, TNA:PRO.

${ }^{134}$ Vgl. Tehran \#2062, 11.4.55; Dulles Letter, 27.6.55; A. Dulles Letter, 1.7.55; State \#55, 11.7.55; Wilson Letter, 5.8.55, FRUS 55-57, XII, S. $729 \mathrm{ff}$., $745 \mathrm{ff}$., 747f., $755 \mathrm{ff}$., 761f.; auch der OCB forderte Entgegenkommen in der Frage der Militärhilfe, da anderenfalls die interne Position des Schahs gefährdet werde: "His position would deteriorate and with it the stability of the Iranian Government."; OCB, Working Group Draft, Progress Report on NSC 5504, 18.5.55, ,OCB 091. Iran (File \#2) (7)‘, WHO, NSCS, OCB Central File Ser., Box 43, DDEL; vgl. PERSSON, Baghdad Pact, S. 252-9. 
amerikanische Wahrnehmung der nahöstlichen Entwicklungen ein weiteres Mal. Symptomatisch für diesen Wandel war die Kehrtwende, die Secretary of State John Foster Dulles hinsichtlich des Bagdadpaktes vollzog. Dulles wandte sich im NSC gegen den Anschluss Irans an den Westen, da ein solcher die Polarisierung in der Region entlang der Frontlinien des Kalten Krieges zementieren würden, mit der Folge eines Überschwenkens der arabischen Führungsmacht Ägypten ins östliche Lager. ${ }^{135}$ Dulles, der ohne explizite amerikanische Zusagen mit einem Zögern der Iraner gerechnet hatte ${ }^{136}$, unterschätzte aber die Entschlossenheit Mohammad Rezās (,,it is a case of now or never [...]“), den Beitritt gegen alle Widerstände durchzusetzen. Angesichts ihrer langjährigen Unterstützung eines iranischen Beitritts war die Eisenhower-Administration letztlich gezwungen, diesen Schritt auch öffentlich gutzuheißen. ${ }^{137}$ Im NSC verteidigte Dulles die eigene Politik und verstieg sich sogar auf die Behauptung, ,[...] the record is clear that the United States had not put pressure on Iran to join the Baghdad Pact at this time and, accordingly, the United States could not be blamed for the consequences. “138

Diese Bemerkungen Foster Dulles im Oktober 1955 waren bereits die ersten Anzeichen für die später auf amerikanischer Seite verbreitete Apologetik der eigenen Politik gegenüber einer nahöstlichen Verteidigungsorganisation nach 1953. Insbesondere das anglo-amerikanische Verhältnis litt unter den nachträglichen Schuldzuweisungen der Verbündeten. Im Dezember 1955 bezichtigte Foster Dulles intern die Briten, die ,[...] had been lately flexing their muscles in this area and, as a result, creating certain problems for the United States." So hätten sie, unter anderem, „pressured the Iranians“ dem Bagdadpakt beizutreten, eine durchaus bestreitbare Behauptung. Hier spiegelten sich die zu diesem Zeitpunkt bereits absehbaren Konsequenzen aus der Gründung des Bagdadpaktes wie z.B. die weitere Polarisierung der Region und die Entstehung einer neuen Front im Kalten Krieg. Die Fehlanlagen in der Bagdadpakt-Konzeption zeigten sich nicht allein in seinem zweifelhaften Nutzen in Anbetracht der tatsächlichen sowjetischen Bedrohung in der Region oder den ,impossible demands on the generosity of the West" seitens der Mitgliedstaaten. Noch gravierender aus Sicht Washingtons aber war, dass der Pakt zu einer Polarisierung und Destabilisierung des Nahen und Mittleren Ostens (und infolge Pakistans Mitgliedschaft auch Südasiens) beitrug und letztlich der UdSSR

\footnotetext{
${ }^{135}$ Dulles definierte die US-Politik explizit als darauf gerichtet, den Waffendeal auf eine „one shot affair“" $\mathrm{zu}$ begrenzen und schlussfolgerte daraus die Notwendigkeit, den Eindruck eines „,major counter-move in Iran“ $\mathrm{zu}$ vermeiden. Siehe 260th NSC Meeting, 6.10.55, FRUS 55-57, XII, S. 163; Dulles zeigte zudem Verständnis für sowjetische Bedenken bez. einer Westorientierung Irans. Moskau habe ,genuine concern“, der Westen beabsichtige das Land als Militärstützpunkt zu verwenden.

${ }^{136}$ Dulles , , [...] replied that it was perfectly O.K. if the Iranians elected to join the Baghdad Pact on their own but he very much doubted that they would do so in the absence of a further quid pro quo from the United States."; ebd., S. 164.

137 Vgl. Tehran \#588, 5.10.55; Telcon, 6.10.55; State \#569, 6.10.55, ebd., S. 778ff., 780f., 782f.; zu den Hintergründen der Entscheidung vgl. Baxter Letter, 7.10.55, ebd., S. 784ff.; Zitat Mohammad Rezās aus Tehran \#599, 8.10.55, ebd., S. 788.

${ }_{138} 261$ st NSC Meeting, 13.10.55, ebd., S. 170; vgl. ASHTON, Hijacking, S. 133f.; tatsächlich gab es britische Offizielle, die den Pakt in erster Linie als Maßnahme gegen Nasser betrachteten: „Egypt is the largest of the Arab states and no Western policy in the Middle East which is actively opposed by Egypt will be entirely satisfactory. An effort should be made to prevent Egypt falling completely under Russian domination. If this fails we must try ruthlessly to isolate Egypt.“, Kirkpatrick Memo, 30.10.55, FO 371/115469, TNA:PRO.
} 
Optionen zur Durchdringung dieser Weltregionen und zur allgemeinen Einflusserweiterung bot. Nach dem Desaster des von London erfolglos betriebenen Beitritt Jordaniens im Dezember 1955, der landesweite Unruhen und den Ausnahmezustand zur Folge hatte, folgten gegenseitige Schuldzuweisungen zwischen London und Washington. „The British have never had any sense“ in ihrer regionalen Politik, monierte der amerikanische Präsident intern, während Eden die Amerikaner direkt des „Appeasement“ gegenüber Nasser bezichtigte. ${ }^{139}$ Die Uneinigkeit zwischen den anglo-amerikanischen Mächten mit Bezug auf Mitgliedschaft und Ausrichtung des Bagdadpaktes im Laufe des Jahres 1955 erzeugte erhebliches Misstrauen zwischen Washington und London bezüglich der Absichten der Gegenseite - die sich hier manifestierende Erosion der „kompetitiven Kooperation“ sollte letztlich in die existenzielle Krise der special relationship in der Suez-Affäre vom Herbst 1956 münden. ${ }^{140}$

Die wankelmütige Politik der Eisenhower-Administration hinsichtlich des Bagdadpaktes führte zudem nicht nur zu Belastungen im Verhältnis zu London. Wie zu erwarten gewesen war, hatte sich gerade Mohammed Rezā große Hoffnungen auf eine Aufwertung der bilateralen Beziehungen zu Washington in Reaktion auf den iranischen Beitritt gemacht, und erwartete nun eine Aufstockung der Militärhilfe an die iranischen Streitkräfte. Die Hoffnungen waren nicht völlig unberechtigt, da sich innerhalb der amerikanischen Regierungsbürokratie die Standpunkte verschoben - so unterstützten die JCS nunmehr nicht nur den zeitnahen Beitritt

${ }^{139}$ Dulles' Zitat aus 271st NSC Meeting, 22.12.55, FRUS 55-57, XII, S. 214; , ,generosity“ in MemCon, 13.1.56, ebd, S. 226; vgl. auch TelCon, 7.4.56, ebd., S. 270; „The Northern Tier was certainly Mr. Dulles's idea, but it has suited our book very well and enabled us to produce a satisfactory revised treaty with Iraq, so that we both worked strongly for the Turco-Iraqi Pact.“; Makins to Kirkpatrick, 5.8.55, FO 371/115585, TNA:PRO; im März 1956 entließ der unter Druck geratene König Hussein schließlich Sir John Glubb Pasha, seit 1939 britischer Kommandeur der jordanischen Arab Legion, ein schwerer Schlag für die britische Position im Nahostgebiet. Zur Jordanienkrise vgl. COHEN, Defending the Northern Tier, S. 95-105; PERSSON, Baghdad Pact, S. 274-80; PODEH, Quest for Hegemony, S. 172-95; ,We had been left with the Turco-Iraqui $\{$ sic; R.P. $\}$, wh $\{$ which $\}$ the Americans started, and then ran out of.“; Eintrag vom 14.7.55, in CATTERALL, Macmillan Diaries: I, S. 449; Eisenhower gab zudem zu, er sei ,[...] a little 'afraid' of the results of the Bagdad \{sic $\}$ Pact.“; Whitman Note, 16.12.55, ,ACW Diary December 1955 (3)', AWF, Ann Whitman Diary Ser., Box 7, DDEL; Eden-Zitat aus Eden Letter to Eisenhower, 5.3.56, in: BOYLE, Eden-Eisenhower Correspondence, S. 119.

140 ,While we could not let the British make our policy for us in this area, or follow the British line blindly, we should certainly cooperate with the British [...]", merkte Dulles noch im November an. Zit. aus 267th NSC Meeting, 21.11.55, FRUS 55-57, XII, S. 202; zum anglo-amerikanischen Misstrauen infolge der Episode vgl. RuAne, Anglo-American Relations, S. 17ff.; LuCAS, Divided We Stand, S. 58-81; AshTON, Hijacking, S. 135ff.; PERSSON, Baghdad Pact, S. 331-6; zusätzlich verschärft wurden die Differenzen durch den schwelenden Territorialkonflikt um die Buraimi-Oase zwischen Saudi-Arabien und den britischen Protektoraten am Persischen Golf. Washington zeigte sich irritiert über die Entscheidung Londons im Oktober 1955, die Frage unilateral militärisch zu lösen - ein hoher britischer Offizieller zeigte sich dagegen „,bewildered“ über die US-Unterstützung für die saudische Politik ,,in their drive to absorb the small pro-western States of South-East Arabia.“; FO to Washington, No. 5998, 15.12.55, FO 371/114559; die Schuld sah man nicht zuletzt auf Seiten ARAMCOs: ,[...] Aramco are probably the greatest obstacle to Anglo-United States harmony in the Middle East and we should, in all our contacts with American oil men, officials and politicians, stress the harm which is done to our mutual interest by Aramco's policy of unrelieved appeasement."; Dodds-Parker to Lloyd, 24.7.56, FO 371/120812, TNA:PRO; vgl. PETERSEN, Conflict and Cooperation, S. 48-75; Eintrag vom 20.10.55, in CATTERALL, Macmillan Diaries: I, S. 493; Eden beschwerte sich im Januar 1956, die saudische Politik spiele Moskau in die Hände. Vgl. Eden Letter to Eisenhower, 16.1.56, ,Dulles, John Foster Jan. '56', AWF, Dulles-Herter Ser., Box 6, DDEL; vgl. Dulles Letter to Macmillan, 6.12.55, BDEE B, 4, III, S. 469ff.; einiges spricht für eine, wenngleich nicht offengelegte, britische Absicht, die Polarisierung in der Region voranzutreiben, um auf diese Art eine amerikanische Parteinahme zugunsten der britischen Position zu erzwingen. Shuckburgh erwähnt in diesem Zusammenhang einen von ihm und Foreign Secretary Harold Macmillan entworfenen „Machiavellian scheme“ in diesem Kontext. Siehe den Eintrag vom 5.10.55, in: ShuckBurgh, Descent to Suez, S. 289; ein angedachtes Konzept war vermutlich der schließlich im März 1956 vom NSC beschlossene Plan OMEGA mit britischer Beteiligung und mit dem Ziel, Nasser zu schwächen bzw. zu stürzen. Vgl. u.a. TAKEYH, Eisenhower Doctrine, S. 105-23; LUCAS, Divided We Stand, S. 104-34. 
der USA zum Bagdadpakt, sondern forderten nun analog auch ein größeres Aufrüstungsprogramm für Iran. ${ }^{141}$ Die formelle Bagdadpakt-Organisation (Middle East Treaty Organization) konstituierte sich auf einer Sitzung der fünf Mitgliedsstaaten in der irakischen Hauptstadt im November 1955- ein permanenter Rat wurde unterstützt von einem militärischen und einem ökonomischen Komitee. Die USA nahmen als Beobachter an der ersten Sitzung teil und etablierten eine permanente politische Liaison mit der Paktorganisation in Bagdad - die JCS sagten zudem informelle Unterstützung bei den militärischen Planungen zu. Das Militärkomitee des Bagdadpaktes definierte Anfang 1956 das Elburs-Hochgebirge als zentrale Verteidigungslinie im Falle eines sowjetischen Vormarsches. Dies geschah wohl in erster Linie aus politischer Rücksichtnahme auf die Iraner, da die bisherige Zāgros-Linie de facto eine Aufgabe des Gros des iranischen Staatsgebietes impliziert hatte. Die Elburs-Entscheidung aber steigerte zugleich die militärischen Anforderungen erheblich und machte den raschen Aufbau der lokalen Verteidigungskapazitäten umso dringlicher. Der Chairman der JCS, Admiral Arthur Radford, argumentierte intern, die US seien verpflichtet, weiter Hilfe zu leisten: ,[...] we have built up a considerable investment in Iran from the standpoint of our national interest." ${ }^{142}$ Angesichts der ungeklärten Rolle Irans im Rahmen der Verteidigungspläne des Bagdadpaktes kam es aber vorerst zu keiner beschleunigten Aufrüstung der Streitkräfte des Landes. Die Größenordnung der US-Militärhilfe an Iran im Rahmen des Mutual Defense Assistance Program (MDAP) - der amerikanische Steuerzahler finanzierte zu dieser Zeit etwa $30 \%$ der iranischen Verteidigung, Budgetzuschüsse nicht eingerechnet - blieb bis 1957 weitgehend unverändert. ${ }^{143}$

\footnotetext{
${ }^{141}$ Die JCS hatten im November 1955 und ein weiteres Mal im März 1956 vergeblich den amerikanischen Beitritt gefordert und eine Aufstockung der Militärhilfe an die Mitgliedsstaaten gefordert. Vgl. CoNDIT, History of the JCS: VI, S. 156f.; eine JCS-Studie vom Oktober 1955 sah nun Militärhilfe für Iran in Höhe von \$192 Millionen für den Zeitraum von 1955 bis 1958 vor. Vgl. JCS History Iran, S. 54f.; auch das State Department forderte vergeblich eine Anpassung in ähnlicher Größenordnung. Vgl. Allen Memo, 28.11.55, FRUS 55-57, XII, S. 20711.

${ }^{142}$ Zitat aus MemCon, 10.2.56, ebd., S. 247; vgl. Baghdad \#987, 20.3.56, ebd., S. 259f.; die Administration lehnte im April 1956 ein weiteres Mal den Beitritt ab - die USA traten aber dem Economic Committee und dem CounterSubversion Committee bei. Vgl. CoNDIT, History of the JCS: VI, S. 156-63; ein US-Beobachter warnte, das ElbursKonzept ,[...] had committed the Baghdad Pact countries to a defense far in excess of what they could pay for."; ebd., S. 158; tatsächlich beruhten die Verteidigungspläne weiterhin vorwiegend auf dem Einsatz von Nuklearwaffen. Allerdings waren britischen Nuklearwaffen für die Region vor 1959 nicht zu erwarten und einen Selbstverpflichtung der USA zur Bereitstellung stand noch aus: ,The present lack of nuclear capability must therefore on no account be revealed", warnten die britischen Chiefs. Siehe , JPS Report to COS, 18.1.56, BDEE B, 4, III, S 482; die britischen Chiefs sahen militärische Vorteile in einer Vorwärtsverteidigung möglichst weit im Norden, ,exploiting to the maximum the effects of nuclear interdiction, SAS operations, terrain and demolitions."; COS Committee Report, 12.10.55, FO 371/115522, TNA:PRO; vgl. COHEN, Defending the Northern Tier, S. 11834; YeşiLbuRSA, Baghdad Pact, S. 131-6, 159-63.

${ }^{143}$ Vgl. oben S. 154, Anm. 126; die MDAP-Lieferungen für die Etatjahre 1954 und 1955 beliefen sich auf etwa \$37 und \$26 Millionen. Für 1956 wurde mit ca. \$23 Millionen gerechnet. Für das Financial Year 1957 (Juli 1956 bis Juni 1957) veranschlagte man \$55 Millionen. Vgl. OCB Study, 10.7.56, ,OCB 091. Iran (File \#4) (3)‘, WHO, NSCS, OCB Central File Ser., Box 44, DDEL; teilweise einzurechnen wären noch der seitens der USA zusätzlich geleistete, Defense Support“, beispielsweise 1955 in Höhe von fast \$80 Millionen für Iran, eine Kategorie, die im Grunde alle auch peripher militärisch relevanten Unterstützungsleistungen mit einschloss, so z.B. auch Zuschüsse zum Ausgleich des Staatshaushalts. Zur Definition vgl. Gleason Note, 31.8.55, FRUS 55-57, X, S. 17f.; ein Interdepartmental Committee, das sogenannte Prochnow Committee, untersuchte eine Reihe von USHilfsprogrammen und kam für Iran zu dem Schluss, dass zusätzliche Militärhilfe von etwa \$230 Millionen zwischen 1957 und 1960 notwendig sei, um die iranischen Streitkräfte in Sollstärke von 145.000 Mann in die Lage $\mathrm{zu}$,limited resistance to a Russian attack“ zu versetzen. Interdepartmental Committee Report, 3.7.56, FRUS 5557, XII, S. 828-836.
} 
Ohnehin lag die amerikanische Schwerpunktsetzung vorerst auf dem politischen Effekt einer Westorientierung der Bagdadpakt-Mitgliedsstaaten. Im Kontext des New Look, ihrer Neuorientierung der amerikanischen Grand Strategy, zeigte die Eisenhower-Administration großes Interesse an allen Aspekten der Countersubversion - in den anglo-amerikanischen Strategiegesprächen im Sommer 1955 verwiesen die US-Offiziellen auf die Möglichkeit einer Stärkung der ,internal security forces“ der regionalen Verbündeten als , ,[...] cheaper way of buying security in the Middle East than building up the security forces. “144 Im Kern war dies eine konzeptionelle Fortführung der permanenten Interventionspolitik, nur dieses Mal nicht gerichtet auf eine pro-westliche Beeinflussung der Führungsschichten, sondern auf eine Unterdrückung anti-westlich bzw. pro-sowjetisch eingestellter Individuen und Gruppierungen, beginnend mit dem sogenannten NSC Action 1290-d-Programm vom Dezember 1954. Diese für die Einleitung einer ambitionierten globalen Antisubversionspolitik grundlegende Entscheidung war bereits mit besonderem Bezug auf kommunistische Infiltration in den Nahen und Mittleren Osten getroffen worden, wobei diese Weltregion zugleich als zentraler Schwachpunkt in der westlichen Verteidigungsstruktur identifiziert worden war. ${ }^{145}$ NSC Action 1290-d wurde anschließend zum Fixpunkt sämtlicher Anstrengungen der USA, den Ländern der „Freien Welt“ Unterstützung zu leisten , ,...] to maintain internal security and to destroy the effectiveness of the Communist apparatus in free world countries vulnerable to Communist subversion.“146 Die 1290-d-Programme, später auch teilweise als „Overseas Internal Security Program“ (OISP) tituliert, umfassten praktische Polizeihilfe wie die Lieferung von Ausrüstung, Trainingsmaßnahmen, aber auch Vermitteln nachrichtendienstlicher Praxis und Unterstützung für politische Kriegführung und Propaganda. Mitte 1956 waren bereits in zehn Ländern amerikanische Polizeiberater tätig, koordiniert von der Civil Police Division innerhalb der International Cooperation Administration (ICA), ab Ende 1957 umbenannt in die orwellianisch betitelte Public Safety Division, und hatten etwa 12.000 einheimische Kräfte ausgebildet. ${ }^{147}$

\footnotetext{
144 MemCon, 23.6.55, ebd., S. 105f;; vgl. auch Hoovers Drängen und die konkreten Vorschläge über die Zusammenarbeit der Mitglieder in MemCons, 24.6.55 und 11.8.55, ebd., S. 117, 150; der britische Assistant Under Secretary of State Evelyn Shuckburgh konzedierte hinsichtlich der Funktion des Paktes, dass ,[...] the need seems less for military might than for the kind of resources required to fight a cold war."; MemCon, 13.1.56, ebd, S. 226; die USA wurden ,participating observer“ des Counter-Subversive Committee des Bagdad-Paktes. Vgl. Newsom Memo, 2.5.56, ,Miscellaneous Originals, 1956, 1957, 1958‘, Lot 61 D 48, Records Relating to the Arabian Peninsula, 1948-1959, NEA/NE, OiCIA, Arabian Peninsula Affairs, Box 15.

${ }^{145}$ Foster Dulles betonte in dieser NSC-Sitzung explizit die herausragende Bedeutung der Region aufgrund der Ölvorkommen: „The Middle East must be denied to the Soviet bloc, for the loss of this area to the free world would be a matter of great gravity."; 229th NSC Meeting, 21.12.54, FRUS 52-54, II, S. 835; interessant an dieser NSC-Sitzung auch die Bemerkung Eisenhowers bezüglich der strategisch übergeordneten Bedeutung Südasiens mit Bezug auf die Nahostregion. Eine Sowjetisierung Indiens habe zwangsläufig einen Verlust des Nahen Ostens zur Folge und müsse daher verhindert werden. Auf britischer Seite wuchs die Besorgnis anlässlich der eigenen Abhängigkeit vom nahöstlichen Öl. Vgl. Macmillan Cabinet Note, 14.10.55, BDEE A, 3, I, S. 135-9; auch die Staff Study zu NSC 5428 vom Juli 1954 erwähnte bereits eine angebliche Abhängigkeit Europas vom nahöstlichen Öl in einer Größenordnung von $90 \%$ im Jahr 1975. Siehe FRUS 52-54, IX, S. $881 \mathrm{f}$.

146 229th NSC Meeting, 21.12.54, FRUS 52-54, II, S. 844.

147 Zahlen und Hintergrund des NSC 1290-d-Programms von RosENAU, Security Assistance, S. 14-27; KuZMarov, Modernizing Repression, S. 9f.; 193-200; für den ähnlichen britischen Ansatz vgl. HASHIMOTO, Security Liaison.
} 
Das Country Team an der Teheraner Botschaft berichtete im April 1955 detailgenau über die interne Sicherheitslage in Iran. Aus Sicht der amerikanischen „Men on the Spot“ hing die fortgesetzte innenpolitische Stabilität des Landes nahezu ausschließlich von der Person des Schahs ab. Mohammad Rezā sei der „bulwark of internal security“ und materielle wie politische Unterstützung seitens der USA dienten, so die Einschätzung aus Teheran, zuvorderst dem Ziel seine Position nach innen zu stärken. Die Botschaft notierte darüber hinaus Defizite im Bereich der Subversionsbekämpfung und der Spionageabwehr und empfahl zusätzliche amerikanische Unterstützung gerade für die Gendarmerie und die nationalen Polizeikräfte. ${ }^{148}$ Als problematisch und ineffizient angesehen wurden vor allem die Konzentration der Countersubversion innerhalb einer kleinen und direkt dem Schah unterstellten Einheit innerhalb der Nationalen Polizei - ,under-staffed, poorly equipped, under-trained, and corrupt“ - und die Übertragung polizeilicher Funktionen an die Armee und die Militärgouverneure. Amerikanische Polizeiberater waren bereits seit 1954 in Iran vor Ort. Zugleich wurden iranische Polizeioffiziere in den USA geschult. Die kleine amerikanische GendarmerieMission war bereits seit 1942 in Iran. ${ }^{149}$ Auf amerikanische Anregung hin wurden 1956 die verschiedenen militärischen und zivilen Antisubversionsaktivitäten im Imperial Staff gebündelt und koordiniert - der Schah entschloss sich schließlich kurz darauf dem amerikanischen Drängen nach einer Überwindung der Rivalitäten zwischen den verschiedenen Diensten mittels der Etablierung eines zentralen zivilen Sicherheitsdienstes nachzugeben. Aufbauend auf der bereits oben erwähnten, nach dem Coup von 1953 aufgebauten nachrichtendienstlichen Zelle, geleitet von Tajmur Baxtijār, wurde noch 1956 der bald berüchtigte SAWAK gegründet Baxtijār wurde der Titel eines stellvertretenden Premiers übertragen und die neue Organisation direkt dem Schah unterstellt. Ein fünfköpfiges CIA-Team blieb bis in die frühen 1960er Jahre im Iran und bildete die gesamte erste Generation des kombinierten In- und Auslandsgeheimdienstes aus. Der SAWAK wurde alsbald zu einem zentralen Mittel staatlicher Repression und war einer der wesentlichen Faktoren für den ansteigenden Anti-Amerikanismus in der Folgezeit. ${ }^{150}$

\footnotetext{
148 Vgl. Tehran \#475, 25.4.55, FRUS 55-57, XII, S. 733-8.

${ }^{149}$ Aus politischen Gründen empfahl man aber keine Abwertung der Antisubversionsfunktionen der Armee und sprach sich auch gegen eine Priorisierung der internen Sicherheit zum Nachteil der allgemeinen Aufrüstung der Streitkräfte aus. Vgl. NSC Study, ,Analysis of Internal Security Situation in Iran pursuant to NSC 1290-d“ [im Folgenden: 1290-d Analysis], 23.8.55/revidierte Version vom 1.12.55, ,OCB 091. Iran (File \#3) (3) ${ }^{6}$, WHO, NSCS, OCB Central File Ser., Box 43, DDEL; vgl. Jernegan Memo, 9.9.55, FRUS 55-57, XII, S. $770 \mathrm{f}$.

${ }_{150}$ Ausführlich bei GASIOROWSKI, Client State, S. 116-21, basierend auf Interviews mit ehemaligen USGeheimdienstlern. Unterstützung leisteten auch die britischen Dienste MI5 und MI6. Das CIA-Team wurde 1960 oder 1961 durch ein Mossad-Team abgelöst, das bis 1965 Ausbildungsmaßnahmen vor Ort durchführte. 1957 wurden die ersten Abhörstationen an der iranisch-sowjetischen Grenze installiert - SAWAK und CIA teilten dabei die gewonnenen Erkenntnisse. Vgl. U.S. Military Information Control Committee MIC 206/171, 7.2.66, Asnād 11, S. 7 ff.; vgl. HASHIMOTO, Security Liaison, S. 870; vgl. das nur teilweise fiktive Werk von DELANNOY, Savak; vgl. auch NAĞĀRI RĀD, Sāwāk, S. 31-5, 41-51; zur Rolle der CIA siehe ebd., S. 59-63; KuZMAROv, Modernizing Repression, S. 193-200; durchsichtig die apologetische Absicht hinter der unwahren Behauptung in POLLACK, Persian Puzzle, S. 77, die Eisenhower-Administration habe Mitte der 1950er Jahre die „,covert action programs““ in Iran geschlossen. Ähnlich die Bagatellisierung der amerikanischen Beteiligung in DAUGHERTY, Shadow, S. 40ff.; der Konsequenzen aus der Kooperation mit dem SAWAK für das Amerikabild in Iran war man sich von Anbeginn an bewusst. Man solle ,[...] exercise caution in order to avoid becoming identified with the political aspects of police measures to the detriment of U.S. prestige in Iran."; Memo to OCB Assistants, 18.6.57, ,OCB 091. Iran (File \#5) (4) ‘, WHO, NSCS, OCB Central File Ser., Box 44, DDEL.
} 
Diese Form der „security assistance“ für die Sicherheitsorgane der verbündeten Staaten war ein zentrales Element der im Vergleich zur vorhergehenden Truman-Administration noch entschlosseneren Fokussierung der amerikanischen Grand Strategy auf psychologische und politische Kriegführung sowie auf verdeckte Operationen. ${ }^{151}$ Eisenhowers „Basic National Security Policy“, zum ersten Mal kodifiziert in der NSC-Studie NSC 162/2 vom Oktober 1953, ermächtigte bereits die verschiedenen Organe ,\{to; R.P.\} [...] take all feasible diplomatic, political, economic and covert measures to counter any threat of a party or individuals directly or indirectly responsive to Soviet control to achieve dominant power in a free world country. " 152 NSC 5412, verabschiedet von Eisenhower im März 1954, wiederholte diese Phrase und beauftragte die CIA mit der Durchführung verdeckter Operationen in diesem Sinne. ${ }^{153}$ Die stärkere Gewichtung geheimdienstlicher Operationen im Kontext des Kalten Krieges war dabei nicht zuletzt eine Folge der verbreiteten Wahrnehmung von TPAJAX 1953 und PBSUCCESS von 1954, einer ähnlichen Operation in Guatemala, als „Erfolge“, die anschließend Vorbildcharakter für die US-Politik in der sogenannten Dritten Welt annahmen. ${ }^{154}$ Was folgte war die häufig als „Golden Age“ titulierte Ära umfassender und zudem nur nachlässig kontrollierter verdeckter Operationen seitens der CIA unter ihrem Direktor Allen Dulles, bald mit einer Schwerpunktsetzung in den gerade erst dekolonisierten Gebieten. ${ }^{155}$ Eine von Präsident Eisenhower persönlich in Auftrag gegebene Studie vom September 1955 über die „,verdeckten Aktivitäten“ des Directorate of Plans der CIA, bekannt als „,Doolittle Report“, kam zu dem Schluss, dass die USA ,[...] are facing an implacable enemy whose avowed objective is world domination by whatever means and at whatever cost.“ Tradierte amerikanische Vorstellungen über „fair play“ müssten aufgegeben werden, um in diesem Wettbewerb bestehen zu können: „We must develop effective espionage and counterespionage services and

\footnotetext{
${ }^{151} \mathrm{Vgl}$. bereits GADDIS, Strategies, S. 154-9; zum militärischen Strategiewandel vgl. LEIGHTON, History of the Office of the Secretary of Defense: III, S. 184-230; spätere Analysen des New Look unterschätzten häufig die Bedeutung der verdeckten Kriegführung für die strategische Gesamtkonzeption.

${ }_{152}$ Zugleich wurden die Regierungsstellen autorisiert, zu ,[...] undertake selective, positive actions to eliminate Soviet-Communist control over any areas of the free world."; NSC 162/2, 30.10.53, FRUS 52-54, II, S. 595.

${ }^{153}$ Lay Note, NSC 5412, ,Covert Operations', 15.3.54, FRUS 50-55: The Intelligence Community, S. 475-8; wie die Vorgängerdirektiven der Truman-Administration NSC 10/2 und 10/5, enthielt auch NSC 5412 wieder eine zusätzliche Fokussierung auf die nicht-kommunistische Welt: , , [...] strengthen the orientation toward the United States of the peoples and nations of the free world."; die Nachfolgestudien NSC 5412/1 und NSC 5412/2 vom März bzw. Dezember 1955 übertrugen die Entscheidungsprozesse über verdeckte Operationen auf jeweils kleinere Formationen, vom OCB über eine kurzlebige Planning and Coordination Group innerhalb des OCB und schließlich auf die Special Group bzw. auch 5412/2 Designated Representatives genannt. Vgl. NSC 5412/1, 12.3.55; NSC 5412/2, o.D. [Dezember 1955], ebd., S. 622-5, 746-9.

${ }_{154}$ Die Kontingenz der vermeintlichen Erfolge und die langfristig negativen Konsequenzen für die globale Stellung der USA zurecht betonend ETGES, All that Glitters; es gab bereits ähnliche zeitgenössische Warnungen. So hatte OIR im State Department gewarnt, die Uneinigkeit der Anti-Mosaddeg-Kräfte mache letztlich eine erfolgreiche Durchführung der Operation unwahrscheinlich. Vgl. CIA [Donald N. Wilber], Clandestine Service History, S. 89f.; ähnlich CIA History Staff, The Battle for Iran, S. 78f.: General Rijāhi hatte zudem die meisten Coupteilnehmer im Vorfeld identifiziert. Hätte er alle verdächtigen Offiziere festnehmen lassen, ,,the coup would never have started."; zudem verfügte er auch über Hinweise auf eine amerikanische Involvierung; lie $\beta$ aber dennoch Automobile der US-Missionen, die Putschisten beförderten, ungehindert und ohne Durchsuchung weiterfahren.

${ }^{155}$ Zur Anwendung der covert operations in der sogenannten Dritten Welt vgl. PrADos, Face of Decolonization; der später DCI Richard Helms, damals im Directorate of Plans, nannte diese Phase die ,high tide of covert action“. Siehe Helms, Look Over My Shoulder, S. 101-20, hier 106; vgl. Callanan, Covert Action, S. 86-108.
} 
must learn to subvert, sabotage and destroy our enemies by more clever, more sophisticated and more effective methods than those used against us. “156

Die Administration autorisierte während ihrer achtjährigen Amtszeit weltweit insgesamt 170 geheime Projekte und Operationen unterschiedlichster Größenordnung. ${ }^{157}$ Auf die anfänglichen Erfolge in Iran und Guatemala folgten alsbald eine ganze Reihe von Fehlschlägen: allein im Nahen und Mittleren Osten die Operationen „Wakeful“ (1956) und „Wappen“ (1957) in Syrien, letztere auch die Ermordung von Führungsfiguren mittels „executive action“ ins Auge fassend, die anglo-amerikanischen Operationen OMEGA bzw. MASK (1956) gegen Nasser, die nur in Umrissen belegbare Operation „SIPONY“ von 1957, wieder den Sturz Nassers intendierend, und schließlich weitere Operationen nach der Revolution im Irak 1958. ${ }^{158}$ „Crypto-Diplomacy“, eine Wortschöpfung des legendären wie umstrittenen amerikanischen Geheimagenten Miles Copeland, schien zu dieser Zeit das bevorzugte Werkzeug der Eisenhower-Administration in ihrer Politikformulierung gegenüber den jungen nahöstlichen Nationen. ${ }^{159}$ Angesichts der vielen Fehlschläge schwand im Laufe der Dekade die Zuversicht in „covert actions“. Der 1956 zum Zwecke der Kontrolle geheimdienstlicher Operationen eingerichtete President's Foreign Intelligence Advisory Board (PFIAB) übte bald scharfe Kritik an den ungezügelten Operationen der CIA. Ein vom PFIAB berufener Panel registrierte im Herbst 1956 die mangelnde Kontrolle der CIA, der Konzeption, Planung und bisweilen letztlich auch die Entscheidungsgewalt über die Durchführung verdeckter Operationen überlassen werde: „The CIA, busy, monied and privileged, likes its 'King Making' responsibility $[\ldots]$ “. . Bis heute verschollen, verwies dieser sogenannte Bruce-Lovett Report auf die vielfältigen und unkontrollierbaren Konsequenzen dieser Operation für die allgemeine amerikanische Außenpolitik - so würden Repräsentanten der CIA (,politically immature“) häufig direkt mit ausländischen Staatsoberhäuptern und Regierungschefs interagieren in Kontakten, die ,[...] are in reality only the continuation of relationships established at a time when the foreign personalities involved may have been 'the opposition'.":

Should not someone, somewhere in an authoritative position in our government, on a continuing basis, be counting the immediate costs of disappointments (Jordan, Syria, Egypt, et al), calculating the impacts on our international position, and keeping in mind the long-range wisdom of activities which have entailed our virtual abandonment of the international 'golden rule', and which, if successful to the degree claimed for them, are responsible, in a great

\footnotetext{
${ }^{156}$ Special Study Group Report, o.D. [September 1955], FRUS 50-55: The Intelligence Community, S. 542f.; die Studie war benannt nach dem Chairman des Panel of Consultants, der die Studie erstellt hatte, Air Force General James H. Doolittle.

${ }^{157}$ Vgl. CIA Study ,Coordination and Policy Approval of Covert Operations‘, 23.2.67, CIA-FOIA.

${ }^{158}$ Unklar ist, ob es sich hier immer um die auch intern benutzten CIA-Kryptonyme handelt - zudem sind nur einige wenige Länder-Digraphen bekannt, wie eben z.B. TP für Iran. Die Briten benannten die "Wakeful“" Operation „Straggle“. Vgl. LitTle, Mission Impossible, S. 666f., 672-82; DORRIL, MI6, S. 606-40, 652-9; RAthmell, Covert Struggle, S. 125-44; Jones, Preferred Plan; LuCAS/Morey, Hidden 'Alliance'; LuCAS/TAKEYH, Alliance and Balance; EvelAnd, Ropes of Sand, S. 247f.; CoPElAND, Game Player, S. 127, 16771; WILFORD, America's Great Game, S. 144f., 189-201, 217-31, 245-76.

${ }^{159} \mathrm{Zu}$ Copeland vgl. ebd., S. 66-73; siehe auch COPELAND, Game Player, S. 196.
} 
measure, for stirring up the turmoil and raising the doubts about us that exist in many countries of the world today? What of the effects on our present alliances? Where will we be tomorrow? ${ }^{160}$

Der alarmistische Ton des Bruce-Lovett Report suggeriert verdeckte Aktionen zu dieser Zeit in einem nur schwer vorstellbaren Ausmaß und vermutlich wird die Zukunft eine ganze Reihe weiterer Operationen ans Licht bringen, von deren Existenz die Forschung zum gegenwärtigen Zeitpunkt noch überhaupt keine Kenntnis besitzt.

Bezüglich Iran wurde seitens der amerikanischen intelligence community die Tudeh weiterhin als hauptsächliche Gefahr für die innere Sicherheit eingeschätzt. Sie sei die ,only disciplined political group“, wohingegen die NWI, die Nachfolgeorganisation der Nationalen Front, wie auch verbliebene ethnische und tribale Widerstandsgruppen als nur begrenzte Gefahr für die Stabilität Irans eingeschätzt wurden. Man schätzte die Stärke der Tudeh Mitte 1955 auf 5.10.000 Mitglieder und bis zu 15.000 Sympathisanten in der Bevölkerung, trotz des Verbots der Partei und ihrer Frontorganisationen seit 1949. Die Repressionsmaßnahmen seitens der Zentralregierung hätten allerdings die Organisationsstrukturen der Partei weitgehend zerschlagen - die Parteiführung war entweder im Gefängnis oder im Exil - und die Tudeh war somit kaum handlungsfähig und zuvorderst mit ihrem Überlebenskampf beschäftigt. ${ }^{161}$ Von herausragender Bedeutung war die Entdeckung und Zerschlagung der bereits im Zusammenhang mit der Ölkrise erwähnten „Offiziersorganisation“ (Sāzemān-e Afsarān) im September 1954, die zum Zeitpunkt ihrer Zerschlagung etwas unter 500 Offizieren umfasste. Sie diente offenbar zuvorderst der Nachrichtenbeschaffung und weniger der tatsächlichen Umsturzvorbereitung. ${ }^{162}$ Das Tudeh-Netzwerk verfügte zwar über keinen Offizier mit einem Rang über dem eines Oberst, wäre aber, so die CIA in einer Studie, theoretisch in der Lage gewesen, den Schah sowie die Mehrheit der Regierung und der Militärführung auszuschalten. ${ }^{163}$ Die anschließenden Prozesse - mehr als 40 der Verurteilten wurden hingerichtet - wurden seitens der amerikanischen Stellen propagandistisch ausgeschlachtet und für Sendungen in ganz Asien verwandt. ${ }^{164}$

\footnotetext{
${ }_{160}$ Coyne Memo to PFIAB, 9 Mai 1961, CIA-Helms;. vgl. auch die Schilderung in SCHLESINGER JR., Robert Kennedy, S. 454-8; anhand seiner Zitate bleibt es unklar, ob Schlesinger über den Original-Report verfügte oder ebenfalls auf das Coyne-Memorandum vom Mai 1961 zurückgriff. Der Bruce-Lovett Report trug im März 1957 zur Erweiterung von NSC 5412/2 durch einen Annex bei, der die Kontrollfunktionen stärkte, ohne das grundlegende Problem der de facto-Autonomie des CIA Directorate of Plans zu lösen. DCI Allen Dulles verzögerte grundlegende Reformen bis zum Ende seiner Amtszeit. Zum Verschwinden des Report vgl. Center for the Study of Intelligence Newsletter, No 3 (Spring 1995), verfügbar auf https://cryptome.org/ic-black5601.htm; mit ,golden rule" ist vermutlich das Nichteinmischungsprinzip gemeint.

${ }^{161}$ Vgl. 1290-d Analysis.

162 Teilweise auch als ,Militärorganisation“ (Sāzemān-e Nezāmi-je Hezb-e Tude-je Irān) bezeichnet. Vgl. XosRouPANĀH, Sāzemān-e Afsarān-e Hezb-e Tude-je Irān; MIYATA, Tudeh Military Network; eine Einschätzung der Stärke bei ABRAHAMIAN, The Coup, S. $214 \mathrm{f}$.

${ }_{163}$ Vgl. CIA, ,The Officer's Organization of the Tudeh Party‘, Januar 1955, CREST: \#RDP78*005-9; Stevens to Eden, No. 72, 15.9.54, FOCP V, B, 7, S. 96-9.

${ }^{164}$ Vgl. F. Bundy Memo, 14.12.54, ,OCB 091. Iran (File \#2) (4)‘, WHO, NSCS, OCB Central File Ser., Box 43, DDEL; angesichts der Zerschlagung geriet die Tudeh anschließend in eine schwere innerparteiliche Krise. Vgl. BEHROOZ, Legacy, S. $123 \mathrm{ff}$.
} 
Erstaunlich war dagegen die Einschätzung der NWI als nur begrenzt bedrohlich angesichts der konstatierten ca. 1.5 Millionen Mitglieder und Sympathisanten. Den nicht weiter definierten xenophoben religiösen Elementen wurde zumindest die Fähigkeit zugeschrieben, große Menschenmassen mobilisieren zu können, doch verfügten sie zu diesem Zeitpunkt nicht über ein kohärentes politisches Programm bzw. waren weitgehend loyal oder zumindest indifferent zur Monarchie eingestellt. Die Stammeskonföderation der Ġašğà i, auf 2-300.000 Mitglieder geschätzt, hatte ihren ohnehin begrenzten Handlungsspielraum eingebüßt, zumal Mohammad Rezā rücksichtslos gegen die Machtposition ihrer der Illoyalität verdächtigten Khane vorging. ${ }^{165}$

Neben ,political action“, den „klassischen“ verdeckten Operationen, war für die EisenhowerAdministration die psychologische Kriegsführung von zentraler Bedeutung. Der Präsident selbst zeigte sich überzeugt, ,[...] public opinion wins most of the wars and always wins the peace. “166 Unmittelbar nach seinem Amtsantritt gab Eisenhower eine umfassende Studie über die amerikanische Gesamtstrategie im Kalten Krieg in Auftrag - das Committee on International Information Activities, besser bekannt als Jackson Committee, empfahl im Juni 1953 eine grundsätzliche Aufwertung der psychologischen Kriegsführung und der Propagandatätigkeit seitens der USA, unter anderem in der Gründung der United States Information Agency (USIA) resultierend. ${ }^{167}$ Die Studie des Jackson Committee empfahl eine Ausweitung der bestehenden amerikanischen Programme zur psychologischen Kriegsführung gegen den sowjetischen Machtbereich, sowohl was „weiße“, „graue“ und "schwarze“ Propaganda betraf. Die Studie enthielt neben den offensiven, gegen den sowjetischen

\footnotetext{
${ }^{165}$ Vgl. 1290-d Analysis; zu den internen Auseinandersetzungen in der NWI und ihrer de facto-Zerschlagung durch den SAWAK bis 1957 vgl. CHEHABI, Liberation Movement, S. 130f., 137-9; die Kurden wurden angesichts ihrer besseren Bewaffnung und des gewachsenen historischen Misstrauens als wahrscheinlicheres Ziel sowjetischer Subversionsanstrengungen betrachtet. Die von der Enteignung bedrohten Ġašğà ${ }^{2}$-Stammesführern mobilisierten ihre Unterstützer in Washington gegen den Schah. William O. Douglas, Richter am Supreme Court, war durch zahlreiche Reisen mit der Lage in Iran vertraut und unterhielt persönliche Kontakte zu Teilen der Opposition und insbesondere zu den Ġašğà $\dot{i}$. Douglas bezeichnete die (nicht gerade liberal eingestellten) Stammesführer als „healthy democratic influence in Iran“ und als Rückversicherung gegen kommunistische Unterwanderung der Armee. Das State Department bestätigte in seiner Reaktion die „many acts of friendship toward the United States“ seitens der Khane, ein Verweis auf die engen Beziehungen der Brüder zur CIA, behandelte die Auseinandersetzung aber als interne Angelegenheit. Vgl. Douglas Letter, 8.3.57; Rountree Letter, 3.6.57, ,Iran Qashqais [1957]‘; Rountree Memo, 8.4.57, ,Memos Thrus S/S for Clearance 1957‘, Lot 60 D 533, Subject Files Relating to Iran, 1951-1958, OiCIA/GTI, Box 10; bereits 1951 hatte Botschafter Grady gewarnt, Douglas sei in Iran eine ,decidedly controversial person“; Grady Letter, 23.5.51; Tehran \#851, 12.101950, ,'G“ Letters', Lot 53 D 468, Office Files of McGhee, Box 2, RG 59; vgl. Herter Letter, 29.5.57, ,Ghashghai, Miscellaneous, 1954-77‘, Box 332, Douglas Papers, LOC; zur Kooperation eines der drei Ġašğā i-Brüder mit der CIA nach der Revolution und seiner Hinrichtung durch die Revolutionsregierung siehe GASIOROWSKI, Overthrow the Islamic Regime?, S. 122f.; siehe auch die feindseligen Einschätzungen Douglas' in ZAHEDI, Memoirs, Vol. II, S. 26ff.; und FARMANFARMAIAN, Blood and Oil, S. $243 \mathrm{f}$.

${ }^{166}$ Zit. aus HIXSON, Parting the Curtain, S. 22; vgl. auch das interessante Dokument zu „weißen“, „grauen“" und „schwarzen“ Propagandaoperationen aus dem Umfeld des OCB. Siehe OCB Paper, 14.5.54, FRUS 50-55: The Intelligence Community, S. 501-5; die britische Botschaft schätzte die nationalistische Opposition - ,a ragged band of malcontents living in the political wilderness and watched closely by S.A.V.A.K." - ebenfalls als schwach ein, sah sie aber dennoch als ernstzunehmenden Repräsentanten der Aspirationen der wachsenden urbanen Mittelschichten. Vgl. Stevens to Lloyd, No. 20, 17.2.58, FO 371/13309, TNA:PRO.

${ }_{167}$ Das Jackson Committee hatte seinen Namen von seinem Vorsitzenden, dem früheren CIA Deputy Director William H. Jackson, erhalten. Vgl. Hixson, Parting the Curtain, S. 22-7; zur Zentralität des Propagandaaspekts in Eisenhowers Konzeption des Kalten Krieges siehe OSGOOD, Total Cold War, S. 46-82; VAUGHAN, Failure of Propaganda, S. 97-127.
} 
Machtbereich bzw. die Volkrepublik China gerichteten Aspekten aber auch diverse Empfehlungen zur Konsolidierung und Verteidigung des eigenen Machtbereichs, euphemistisch immer als „Freie Welt“ tituliert, mit der Zielsetzung ,[...] to persuade foreign peoples that it lies in their own interest to take actions which are also consistent with the national objectives of the United States. "168 Ein ehemaliger, im Nahen Osten aktiver CIA-Agent spezifizierte in seinen Memoiren die Entdeckung und Förderung von effektiven ,agents of influence“ - Führungspersönlichkeiten in allen gesellschaftlichen Bereichen ,[...] whose personal aims and desires fit nicely into what we want" - als zentralen Bestandteil der politischen Kriegführung. ${ }^{169}$ In ihrer Gesamtheit ähnelte der Bericht des Jackson Committee einer umfassenden amerikanischen Doktrin für die Führung des Kalten Krieges jenseits der rein militärischen Dimensionen. Bezüglich der im Zuge der fortschreitenden Dekolonisation entstandenen „neuen“ Staaten empfahl der Bericht eine Schwerpunktsetzung auf die Bekämpfung des Neutralismus. ${ }^{170}$ Eisenhower selbst hatte kurz nach seinem Amtsantritt mit Bezug auf die nahöstliche Welt sein Unbehagen ausgedrückt, ,[...] that we seemed unable to get some of the people in these down-trodden countries to like us instead of hating us." ${ }^{171}$

Das ausgeweitete amerikanische Informationsprogramm in Iran diente zuvorderst dem Zweck, die Reputation des Schahs und der Monarchie gegenüber der eigenen Bevölkerung zu stärken und die pro-westliche Orientierung weiter zu vertiefen, unter anderem mit einer Kulturoffensive auf die iranische Jugend, genannt „Operation Schoolhouse“. Die USIA produzierte die einzige Nachrichtensendung im Filmformat in Iran, der US-Propagandasender Voice of America übertrug zwei Stunden pro Tag und darüber hinaus hatten ICA und USIA der iranischen Regierung einen 50 Kilowatt-Radiosender zur Verbreitung der eigenen Propaganda übergeben. Allein im Etatjahr 1955 hatten zudem 35 Iraner am Austauschprogramm in den USA teilgenommen, darunter 15 ,leaders“, also Personen in Führungspositionen bzw. mit dem Potential, solche in Zukunft einzunehmen. Prononcierter war nun aber auch der Ruf nach umfassenderen wirtschaftlichen und sozialen Reformmaßnahmen und einer konsequenteren Bekämpfung der Korruption, und damit der eigentlichen Ursachen der ,national frustration“. In diesem Sinne wurde es auch als wichtig angesehen, einen modus vivendi mit den

\footnotetext{
168 Report to the President by the President's Committee on International Information Activities [Jackson Committee Report], 30.6.53, FRUS 52-54, II, S. 1795-1899; in der FRUS-Version sind weite Teile des Report gesperrt - so fehlt das „operative“ Kapitel 6 (,Covert Operations Within the Free World“) in Gänze, was einen nachdrücklichen Effekt auf die historiographische Einordnung hatte. Zusätzliche Freigaben in der Version aus dem Jahr 2011 als Teil der Helms Collection. Zitat in ebd., S. 58; zu den Empfehlungen für die Ausweitung des Propagandakrieges gegen den Ostblock vgl. ebd., S. 32-48 (1823-35 in der FRUS-Version).

${ }^{169}$ COPELAND, Game Player, S. 182 \{Hervorheb. i. Orig.\}; Copelands umstrittenen Enthüllungen korrespondieren zumindest hier mit der amerikanischen Konzentration auf bestehende und zukünftige Führungsgruppen. Siehe die Bemerkungen oben zu den NSC-Studien NSC 129/1 vom April 1952 und NSC 5428 vom Juli 1954. Vgl. OSGOOD, Total Cold War, S. 132f.; analog befürchtete die Administration, sowjetische Hilfsprogramme für Ägypten könnten Moskau in Kontakt zu solchen Gruppen setzen: ,[...] we must keep the Russians from getting contacts that enable them to run the country."; MemCon], 13.1.56, FRUS 55-57, XII, S. 233.

${ }^{170}$ Vgl. Jackson Committee Report, S. 56; PARKER, Hearts, Minds, Voices, S. 92-115.

171 135th NSC Meeting, 4.3.53, FRUS 52-54, X, S: 699.
} 
Parteigängern der Nationalisten zu finden. ${ }^{172}$ Die zweite Iran-Studie der EisenhowerAdministration, NSC 5504 vom Januar 1955, enthielt Handlungsanweisungen hinsichtlich Propaganda und der Manipulation der öffentlichen Meinung in Iran, mit besonderem Fokus auf die nationalistische Opposition: „Recognize the strength of Iranian nationalist feeling, try to direct it into constructive channels and be ready to exploit any opportunity to do so, bearing in mind the desirability of strengthening in Iran the ability and desire of the Iranian people to resist Communist pressure.“173

Tatsächlich mehrten sich bald die Rufe nach größerer Aufmerksamkeit für die oppositionellen Kräfte und einem Kurswechsel in der amerikanischen Iranpolitik, insbesondere einer Revision der einseitigen Unterstützung für Mohammad Rezās Dominanz im politischen System Irans. Der Schah hatte schließlich im April 1955 die unpopuläre Zāhedi-Regierung entlassen. Sein Nachfolger Hosejn ‘Alā war ein enger Vertrauter Mohammad Rezās, was allgemein als direkte Übernahme der Regierungsgeschäfte durch den Hof interpretiert wurde. Anfangs seitens der US-Planer als Chance für eine konsequente Umsetzung des Wirtschafts- und Aufbauprogramms für Iran angesehen und mit ,virtually all-out support“ bedacht, wurde die Ergreifung von ,near-dictatorial powers“ seitens des Monarchen alsbald kritisch gesehen: „His government's inability to carry out its announced economic and administrative reform program has seriously affected his prestige.“174 Anstelle der erhofften konsequenten Reformpolitik und politischen Öffnung verfolgte der Schah eine noch stärkere Konzentration der politischen Macht im Hof, was zu einem letztlich ergebnislosen Konflikt mit den traditionell den Mağles dominierenden landbesitzenden und konservativen Kräften führte. Statt die entfremdeten, zuvor Mosaddeg unterstützenden liberalen und nationalistischen Kräfte einzubinden, wie von amerikanischer Seite anfangs erhofft, wandte sich Mohammad Rezā sogar kurzzeitig in populistischer Manier dem reaktionären Klerus zu, dabei eine gewaltsame Kampagne gegen die als häretisch diffamierte religiöse Minderheit der Bahai im Jahr 1955 tolerierend. ${ }^{175}$ Die CIA

\footnotetext{
172 Vgl. 1290-d Analysis; das Jackson Committee hatte explizit eine Ausweitung der Exchange-Programme eingefordert. Vgl. Jackson Committee Report, S. 69-71; Gesandtschaft Teheran an AA, 5.10.54, B 11/1541, PAAA; zur britischen Unterstützung der politischen Kriegführung vgl. WAINWRIGHT, Equal Partners?.

${ }^{173}$ NSC 5504, 15.1.55, FRUS 55-57, XII, S. 689-96, hier 695f.; die fehlenden Paragraphen 31 bis 33 befassen sich analog zur Vorgängerstudie wieder mit Ernstfallszenarien eines sowjetischen Einmarsches bzw. einer versuchten kommunistischen Machtübernahme und wohl auch wieder der Unterstützung der US-Ziele durch ,,covert actions“. Paragraph 32 und 33 vollständig in OCB Draft Report (NSC 5504), 8.6.55, ,OCB 091. Iran (File \#2) (8) ${ }^{\star}$, WHO, NSCS, OCB Central File Ser., Box 43; 1956 wurde beispielsweise der Präsident der Teheraner Universität, Manučehr Eğbāl, mit einem ,leader grant" in die USA eingeladen. Eğbāl wurde kurz darauf zum Hofminister ernannt und kündigte an, er werde für diese Aufgabe „,count heavily on the friendship of the U.S.“; Egbāl war später von 1957-60 Premierminister. OCB Study, 10.7.56, ,OCB 091. Iran (File \#4) (3)‘, WHO, NSCS, OCB Central File Ser., Box 44, DDEL; zum langfristigen Scheitern anglo-amerikanischer antikommunistischer Propaganda in der nahöstlichen Weltregion vgl. VAUGHAN, Failure of Propaganda.

174 OCB Working Group Draft, Progress Report on NSC 5504, 12.6.56, ,OCB 091. Iran (File \#4) (1)`, WHO, NSCS, OCB Central File Ser., Box 44, DDEL; ,,support“ in Gustin Memo, 20.7.56, ,OCB 091. Iran (File \#4) (4)‘, ebd.; vgl. Tehran \#2048, 8.4.55, FRUS 55-57, XII, S. 726-9; COTTAM, Iran and the United States, S. 113, bewertet die Entlassung Zāhedis bündig: „From that point until 1978, the shah was dictator of Iran.“; ZAHEDI, Memoirs, Vol. I, S. 270ff., behauptet, der Widerstand seines Vaters gegen den Beitritt zum Bagdadpakt habe das Zerwürfnis ausgelöst und schließlich seine Ablösung erwirkt.

175 Offenbar bewilligte Mohammad Rezā persönlich die brutale Kampagne auf Anregung Ājatollāh Boruğerdis und der Geistlichkeit - die Besitztümer der Bahai in vielen Städten wurden konfisziert, Offizielle wie der zukünftige SAWAK-Direktor Tajmur Baxtijār beteiligten sich persönlich an der Zerstörung der Heiligtümer. Vgl.
} 
warnte umgehend vor einer , $[\ldots]$ reemergence of reactionary religious pressures and emotions as an unsettling influence in the country. "176 Die Aufgabe der von seinem Vater geerbten antiklerikalen Politik währte allerdings nur kurz - nach einem missglückten Attentat auf Ministerpräsident 'Alā im November wurde die islamistische Terrorgruppe der Fedāeijān-e Eslām zerschlagen. Einige ihrer Führer wurden vor Gericht gestellt und vier von ihnen, darunter ihr Gründer Nawwāb Safawi, zum Tode verurteilt und anschließend hingerichtet. Der Inspirator des aktivistischen und gewaltbereiten Islamismus, Ājatollāh Kāšāni, und diejenigen Nationalisten, die noch vor dem Coup die Seiten gewechselt hatten und zu diesem Zeitpunkt noch politisch aktiv waren, wurden anschließend der Beteiligung an der Ermordung des Ministerpräsidenten 'Ali Razmārās im Jahr 1950 bezichtigt und zeitweilig ebenfalls festgesetzt. ${ }^{177}$

Die Stimmung in Washington kippte mit den Wahlen zum 19. Mağles im April 1956. Massiv manipuliert vom Hof, ähnelte die neue Kammer der bisherigen in ihrer äußerst konservativen Zusammensetzung, wenngleich die Parteigänger des Schahs nun über diejenigen des entlassenen Premiers Zāhedi dominierten (,flagrantly rigged to reelect the Shah's reactionary group“). Die Hoffnungen der Irankenner in Washington, „our continued active support of the Shah“ könne so etwas wie einen, ,[...] short-cut towards a broader base of political power in Iran“" erwirken, waren endgültig als Hirngespinst entlarvt. Innerhalb des OCB bildete sich nun eine Fraktion, die darauf drängte, den amerikanischen Einfluss zugunsten der Umsetzung eines tatsächlichen und umfassenden Reformprogramms in Iran zu nutzen, um den ,,increasing signs of instability in Iran“ frühzeitig entgegenzuwirken. ${ }^{178}$ Voraussetzung für den Erfolg einer solchen Unternehmung - darüber herrschte weitgehende Einigkeit - war amerikanische Bereitschaft „,[to] interfere more directly in the economic sphere“ im Zusammenwirken mit einer starken und unabhängigen Regierung, gleichbedeutend mit einem erzwungenen Rückzug Mohammad Rezās aus der Regierungsverantwortung, wenngleich der Fortbestand der Monarchie nie ernsthaft in Frage gestellt wurde. ${ }^{179}$ Eine Fortsetzung der politischen Dominanz

CHOUBINE, Suppression of the Baha 'is; vgl. GASIOROwSKI, Client State, S. 169ff.; ANSARI, Modern Iran, S. 143f.; AKHAVI, Religion and Politics, S. 76-90.

${ }_{176}$ CIA, ONE, Staff Memo No. 64-55, 23.9.55, CREST: \#RDP79*021-3; vor dem Staatsstreich von 1953 hatte die CIA offenbar auf Betreiben des historisch kundigen Donald Wilber hin noch einen religiös motivierten Aufstand gegen die vorgeblich mit der Tudeh unter einer Decke steckende nationalistische Regierung geplant. Der Plan beinhaltete sogar das Angebot eines Ministerposten ohne Portefeuille an Ājatollāh Boruğerdi und die Inkraftsetzung eines Artikels der iranischen Verfassung, der die Überprüfung der gesamten Gesetzgebung auf Vereinbarkeit mit dem Islam durch ein Gremium bestehend aus fünf Geistlichen vorsah. Vgl. CIA [Donald N. Wilber], Clandestine Service History, Appendix A, S. 4f.; die starke Konzentration auf eine Mobilisierung der religiösen Rechten wurde in der zweiten internen CIA-Geschichte recht kritisch auf Wilbers persönliche Ansichten zurückgeführt, zumal sie vom ,field“ nie umgesetzt worden sei. Vgl. CIA History Staff, The Battle for Iran, S. 35. 177 Vgl. Tehran \#611, 23.1.56, 788.00/1-2356, CF 55-59; Kāšāni versuchte vergeblich vor seiner Verhaftung mit der US-Botschaft Kontakt aufzunehmen. Vgl. TAGHAVI, Fadaeeyan-i Islam, S. 155f.; Mozaffar Bagā ${ }^{-}$i, Hosejn Makki oder Abu 1-Hasan Hā"erizādeh wurden auf Basis der unter Folter zustande gekommenen Aussagen der verurteilten Fedā' ijāan verhaftet. Hā'erizādeh hatte im Mağles die Beteiligung des Hofs am Attentat behauptet. Bez. der Korrektheit dieser Beschuldigung siehe oben S. 100.

${ }_{178}$ Gustin Memo, 20.7.56, ,OCB 091. Iran (File \#4) (4)‘, WHO, NSCS, OCB Central File Ser., Box 44, DDEL; zentral für die Debatten war ein Progress Report vom Juli 1956. Vgl. OCB, NSC Progress Report on NSC 5504, 25.7.56, Iran (7)', WHO, NSCS, Disaster File Ser., Box 66, DDEL; vgl. CHEHABI, Liberation Movement, S. $141 \mathrm{f}$. $\left.{ }_{179},[\ldots]\right]$ nothing would be gained by forcing the present Shah to abdicate in favor of some other member within the royal family, or for Iran henceforth to be governed as a republic."; ebd.; sollten die USA damit fortfahren, 
des Schahs aber war aus amerikanischer Sicht inakzeptabel - die noch unter Henderson dem Schah so ergebene US-Botschaft sandte nun ein vernichtendes Fazit zu dessen Führungstalent: er changiere zwischen „military dictatorship, cynicism and cowardice“. ${ }^{180}$

Der hier entstehende reformistische Interventionismus sollte bis in die frühen 1960er Jahre ein wirkmächtiger Bestandteil der amerikanischen Iranpolitik bleiben. Die Beratungen über ein stärkeres US-Engagement im Laufe des Jahres 1956 zeigten aber beispielhaft die Problematiken und Beschränkungen einer solchen Intervention in Klientelstaaten wie Iran - trotz der bestehenden gewaltigen Machtasymmetrie. Der NSC Planning Board erstellte eine Bestandsaufnahme von Alternativen für die bestehende amerikanische Politik gegenüber Iran. Rasch kam man zu dem Schluss, dass eine Aufgabe des amerikanischen Engagements in Iran angesichts der strategischen Bedeutung des Landes und der Aufrechterhaltung der eigenen Glaubwürdigkeit gegenüber anderen Allianzpartnern nicht realistisch war. ${ }^{181}$ Somit empfahl der Board eine Erhöhung des eigenen Einsatzes mit dem Ziel einer umfassenden Reformierung und Entwicklungsförderung Irans ,[...] pending the day when it is politically and strategically possible for the U.S. to extricate itself from the present deep involvement in Iranian affairs. "182 Vorerst aber war aus Sicht der US-Planer eine Intensivierung des eigenen Engagements geboten, mit dem Ziel einer wirtschaftlichen, sozialen und politischen Stabilisierung Irans. Die politische Beruhigung der Lage sollte allerdings nicht durch breitere Partizipation und Liberalisierung erreicht werden, sondern durch Wiederherstellung der stabilisierenden Bedeutung der Institution der Monarchie, zuvorderst durch einen erzwungenen Rückzug Mohammad Rezās aus dem politischen Tagesgeschäft und die Berufung einer starken Regierung. ${ }^{183}$ Mit einem Programm „subtiler Einflussnahme“ gemeinsam mit Zusagen erhöhter Wirtschafts- und Militärhilfen sollte der Schah zu diesem Rückzug und zur Ernennung

ausschließlich die „reaktionären Elemente“ in Iran zu unterstützen, ,[...] the chaotic conditions that led to the Mosadeq excesses could easily and quickly return“, warnte der OCB. OCB Working Group Draft, 18.5.55, ,OCB 091. Iran (File \#2) (7)‘, WHO, NSCS, OCB Central File Ser., Box 43, DDEL.

${ }^{180}$ Die politische Führung des Schahs , has been a notable failure“. Kitchen Memo, 17.5.56, FRUS 55-57, XII, S. 817; dieses Memo des GTI Deputy Director Jeffrey Kitchen kann als Ausgangspunkt des reformistischen Interventionismus betrachtet werden. Ähnlich die Einschätzung des britischen Botschafters Roger Stevens: ,Good intentions coupled with weak execution resulted in an intermittent and hesitant dictatorship."; Stevens to Lloyd, No. 14, 18.1.57, FO 371/127071; Stevens befürchtete allerdings für den Fall eines Zurückdrängens der politischen Dominanz des Schahs eine rasche Destabilisierung: ,[...] things would be likely to bog down rapidly in complete inertia or to slide towards revolution."; Stevens to Lloyd, No. 104, 29.9.56, PREM 11/2032, TNA:PRO.

${ }^{181}$ Der GTI Desk Officer Owen T. Jones verwies auf das ,heavy U.S. political commitment (in the eyes of the Middle East, at least) to the success of the post-Mosadeq regime which, in a very real sense, owes its continued existence, during the last three years, to the United States."; Annex A to OCB Draft, 24.9.56, ,OCB 091. Iran (File \#4) (7)', WHO, NSCS, OCB Central File Ser., Box 44, DDEL.

182 OCB Working Group Study ,Iran', 22.8.56, ,OCB 091. Iran (File \#4) (6) , ebd.; einen Monat später stellte die Working Group nach weiteren Beratungen fest, es sei ,[...] highly inadvisable for the U.S. to withdraw from its present involvement in Iranian affairs.“; stattdessen empfahl sie mehr Engagement mit dem Ziel der ,maintenance of western initiative in Iran".

${ }^{183}$ Der CIA-Vertreter im Planning Board betonte, das Prestige der Monarchie in Iran habe abgenommen ,to the extent that whereas formerly it represented a major stabilizing element on the Iranian scene, it is rapidly becoming the focal point of resentment [...]."; angesichts der Unfähigkeit des dominanten Schahs würde die Monarchie ihren ,process of self-destruction“ beschleunigen. Annex B to OCB Draft, 24.9.56, Anm. 181. 
eines zentralen Wirtschafts- und Planungskoordinators bewegt werden, der de facto unter amerikanischer Anleitung agieren würde. ${ }^{184}$

Im NSC Planning Board waren die Einschätzungen gegenüber den Führungsqualitäten Mohammad Rezās sehr kritisch, doch sah man die Alternativen einer erzwungenen Abdankung zugunsten eines Verwandten bzw. der Umwandlung Irans in eine Republik als kaum vereinbar mit den amerikanischen Sicherheitsinteressen an. Allerdings mehrten sich die Stimmen, die sich dafür aussprachen ,quiet preparations“ einzuleiten , ,[... for dealing with non-communist elements, including nationalists, who, when a favorable opportunity appears, may come to power." ${ }^{185}$ Letztlich sollten nur Teile dieses reformistischen Impulses umgesetzt werden, wieder einmal den Einflüssen der regionalen Dimension geschuldet. Die Suezkrise von 1956 hatte den Effekt einer erneuten Aufwertung des Bagdadpaktes: GTI im State Department verwies darauf, dass die Krise ,[...] highlights the fact that Iran and the Pact could be built up (if the U.S. chooses to do so) as an invaluable counterpoise to mounting anti-Western hostility in the dangerously disturbed Arab world. “186 Der Wunsch nach Reform trat alsbald wieder hinter die Notwendigkeit von (kurzfristiger) Stabilität zurück. Die CIA wies die Behauptung einer grundsätzlichen Instabilität in Iran zudem als überzogen zurück - es gebe zweifellos eine „unhealthy situation“ in dem Land, aber die Unzufriedenheit habe , ,...] not crystallized into real opposition, if only because of the effectiveness of the Shah's security services in keeping the lid on."187 In dieselbe Richtung zielte ein neues NIE zu Iran im Januar 1957. Das gegenwärtige Regime könne sich mittels Polizeikräften und Armee an der Macht halten „over the next few years" - mittelfristig aber, so konzedierten die geheimdienstlichen Analysten, seien erfolgreiche Reformen und eine erfolgreiche Wirtschaftspolitik unausweichlich. ${ }^{188}$ Die CIA machte dabei unmissverständlich klar, dass die fortgesetzte Westorientierung Irans nur gewährleistet war, solange Mohammad Rezā ,retains control“. ${ }^{189}$

Befürworter einer Fortsetzung bzw. Intensivierung des bisherigen Ansatzes in der amerikanischen Iranpolitik setzten sich in der innerbürokratischen Auseinandersetzung gegen die reformistischen Interventionisten durch, zumal letztere meist in den mittleren und unteren Rängen der Administration zu finden waren. In den oberen Rängen aber hielt man an der

\footnotetext{
184 Weitere Ziele waren die Entlassung der vielen zweifelhaften Berater am Hof und eine weniger offenkundige Manipulation der Wahlen. Zentral war die Idee des eigenen Einflusses auf den Koordinator ,by placing high level U.S. advisers, supported by competent technical teams, in a position where they are authorized to guide and assist [...].“; ebd.; rasche Erfolge und Visibilität sollten durch eine Reihe von ,impact-type projects“ erzielt werden.

${ }_{185}$ Man rechnete für den Fall einer Abdankung bzw. Abschaffung der Monarchie mit einem militärischen Staatsstreich nach ägyptischem Vorbild. Zitat ebenfalls aus ebd.; ähnlich bereits in Kitchen Memo, Anm. 180.

${ }^{186}$ Annex A to OCB Draft, 24.9.56, Anm. 181; Hintergründe der Studie in Gustin Memo, 3.10.56, ,OCB 091. Iran (File \#4) (8)‘, WHO, NSCS, OCB Central File Ser., Box 44, DDEL.

${ }^{87}$ Amory Jr. Memo, 2.10.56, , OCB 091. Iran (File \#4) (8)', WHO, NSCS, OCB Central File Ser., Box 44, DDEL; Jackson wiederholte die CIA-Bewertung fast wörtlich im NSC. Siehe 299th NSC Meeting, 4.10.56, FRUS 55-57, XII, S. 850f.

${ }^{188}$ Vgl. CIA, NIE 34-57, 23.1.57, ebd., S. 875.

189 Ebd., S. 875 \{Hervorheb. V. Verf.\}; andere Quellen widersprachen der CIA-Position einer grundsätzlichen Loyalität der Sicherheitskräfte zum Regime. Die Botschaft berichtete über eine Zunahme an Putschgerüchten in Teheran. Vgl. Tehran \#1130, 23.1.57, ebd., S. 880f.; Tehran \#588, 14.1.56, 788.00/1-1456, CF 55-59.
} 
grundsätzlichen Unterstützung für autoritäre Regierungen, solange sie pro-westlich orientiert waren, fest. Mit Bezug auf Lateinamerika bemerkte ein US-Offizieller unwidersprochen im NSC: „We should [...] stop talking so much about democracy, and make it clear that we are quite willing to support dictatorships of the right if their policies are pro American." ${ }^{190}$ Die dritte NSC-Studie der Eisenhower-Administration zu Iran, NSC 5703/1 vom Februar 1957, hielt dementsprechend an der Unterstützung der weitgehend uneingeschränkten Führungsrolle Mohammad Rezās fest, zuvorderst mittels einer weiteren Steigerung der amerikanischen Wirtschafts- und Militärhilfe: „U.S. political and financial assistance are important pillars supporting the Shah in his present paramount position."191 Gleichzeitig überdauerte der Reformismus in abgeschwächter Form. Zentral für die pro-westliche Stabilität des Landes, so die NSC-Studie, seien spürbare ökonomische Verbesserungen und Maßnahmen zur politischen Integration der „liberalen, nationalistischen und intellektuellen Elemente“ in der Bevölkerung, wobei der Charakter der letzteren und deren Wunschvorstellungen nicht im Detail ausgeführt wurde. Die Protektion des angedachten reformerischen Maßnahmenpakets vor der ,personal inconstancy and arbitrariness“ des Monarchen - eine Chiffre für eine Beendigung des Einflusses des Hofs auf die Regierungsgeschäfte - fand ebenfalls Erwähnung. Wirtschaftliche und politische Maßnahmen dieser Art wurden aber zugleich abhängig gemacht von der Erfüllung der Erwartungen Mohammad Rezās bezüglich der Stärkung der allgemeinen Sicherheit Irans, ein inhärenter Widerspruch, der in der Studie nie aufgelöst wurde. ${ }^{192}$ Entsprechend standen während der Beratungen im NSC über die Verabschiedung der Studie auch die Hilfsprogramme und nicht die Reformvorschläge eindeutig im Vordergrund. Secretary of State Foster Dulles verwehrte sich explizit gegen jede Senkung der US-Hilfe und gegen echten Druck auf die Führung in Teheran - sollten die USA sich auch nur für drei Monate Gesprächen über Hilfsleitungen verweigern, so Dulles, ,[...] we will certainly lose Iran.“193

\footnotetext{
${ }^{190}$ 229th NSC Meeting, 21.12.54, FRUS 52-54, II, S. 838; Eisenhower selbst unterbrach den Sprecher, Secretary of the Treasury Humphrey, und merkte an: ,[...] you mean they're OK if they're our s.o.b.'s.[sons of bitches; R.P.]“ \{Hervorheb. i. Orig.\}; Eisenhower zitierte dabei, offenkundig bewusst, eine kolportierte Bemerkung Franklin D. Roosevelts über den nicaraguanischen Diktator Anastasio Somoza García. Vgl. SCHMITZ, Right-Wing Dictatorships, 1921-1965, S. 4, 313.

${ }^{191}$ NSC 5703/1, ,U.S. Policy Toward Iran‘, 8.2.57, FRUS 55-57, XII, S. 900-10, hier 904; hier auch der Verweis auf die generelle Bedeutung der Hilfsprogramme für die eigene Iranpolitik. So sei das technische Hilfsprogramm bedeutend , $[. .$.$] as a means of making the presence of the United States felt at all levels of the population and$ throughout the country."; ebd.

${ }^{192}$ Vgl. ebd., S. 905f.; siehe auch die angemahnten Reformschritte im Paragraph 21 der Studie in ebd., S. 907; aus den vorhandenen Dokumenten ist es nicht möglich, den Urheber des Konnex zwischen reformistischen Forderungen und Erfüllung der Aufrüstungswünsche zu bestimmen. Der Planning Board nannte bereits im Vorfeld eine glaubwürdige Sicherheitsgarantie eine ",condition necessary to any effective U.S. influence“ auf Reformschritte, gefordert in einem Entwurf des State Departments. Zit. aus Planning Board Memo, 14.1.57, ,Iran (2) ${ }^{6}$, WHO, NSCS, Special Staff File Ser., Box 4, DDEL; der Planning Board selbst verstärkte den Konnex zusätzlich. Möglicherweise verstecken sich konkrete reformistische Maßnahmen im weiterhin nicht freigegebenen Paragraphen 23. Wahrscheinlicher aber ist hier, analog zu den Vorgängerstudien, ein Maßnahmenpaket im Falle verschiedener Szenarien eines kommunistischen Umsturzversuches enthalten. Ein Planning Board-Dokument erwähnt einen solchen Teil über „Communist Seizure of Power or Soviet Invasion“. Vgl. Planning Board Briefing Paper, 22.1.57, ebd.

${ }^{193}$ Vgl. 312th NSC Meeting, 7.2.57, FRUS 55-57, XII, S. 898; mit den Quellen unvereinbar die Behauptung bei BARRETT, Greater Middle East, S. 80-4, 367 n2, die Eisenhower-Administration habe schwerpunktmäßig vor 1958 auf Reformen gedrängt. Ähnlich unhaltbar COLLIER, Nature of Influence, S. 166-9.
} 
Konkret schlug NSC 5703/1 eine Aufwertung der Militär- und Wirtschaftshilfe an Iran vor. Militärhilfe in Höhe von \$185 Millionen für die nächsten drei Jahre sollte die Iraner in die Lage versetzen, im Falle eines sowjetischen Einmarsches „defensive delaying actions“ im Norden, gemeint war hier wieder das Elburs-Gebirge, zu unternehmen. ${ }^{194}$ Trotz stetig steigender Öleinnahmen - nach \$160 Millionen im Etatjahr 1957 erwartete man einen Anstieg auf etwa \$200 Millionen in 1958 - empfahl man auch eine Fortsetzung der Wirtschaftshilfe und implizit auch der die bilateralen Beziehungen seit längerer Zeit belastenden Budgetzuschüsse. ${ }^{195}$ Die International Bank for Reconstruction and Development (IBRD) hatte zudem Iran im Januar 1957 ein Darlehen für die Unterstützung der wirtschaftlichen Entwicklung in Höhe von \$75 Millionen gewährt. ${ }^{196}$ Im August 1957 konkretisierte der NSC die Hilfszusagen im Rahmen von NSC 5703/1 und reduzierte die eingeplante Militärhilfe um etwa \$50 Millionen, wohl auch auf zunehmende Kritik im US-Kongress am verschwenderischen Hilfsprogramm für Iran reagierend. ${ }^{197}$

\footnotetext{
${ }^{194}$ Analog zu dieser Denkweise hatte die Administration bereits unmittelbar nach Suez eine Steigerung der USUnterstützung an Iran auf \$45 Millionen genehmigt, um den wichtigen Verbündeten zu stabilisieren. Vgl. Dulles Memo, 7.12.56, FRUS 55-57, XII, S. 859f.; vgl. dazu NSC 5703/1, S. 906; Annex C zu NSC 5703/1 enthielt auch Vorgaben für eine Reorganisation der iranischen Streitkräfte auf Basis von sechs vollwertigen und sechs Divisionen mit reduzierter Stärke sowie fünf unabhängigen Brigaden, ebenfalls mit reduzierter Stärke bei gleichbleibender Gesamtsollstärke von 152.000 Mann. Vgl. ebd., S. 908ff.; Jones Memo, 11.1.57, ebd., S. 871f.; die Reorganisation erfolgte auf Anraten des ARMISH-MAAG Chiefs Major General J.F.R. Seitz. Vgl. JCS Memo, 30.11.56, ebd., S. 857ff.; JCS History Iran, S. 55ff.; die empfohlene Steigerung der Militärhilfe ging auf Empfehlung des NSC Planning Board über die ursprünglich vom Prochnow Committee - beauftragt mit einer umfassenden Untersuchung der wichtigsten Hilfsprogramme - empfohlenen Richtwerte hinaus. Zum Prochnow Committee vgl. oben S. 158, Anm. 143; vgl. Planning Board Briefing Paper, 5.2.57, FRUS 55-57, XII, S. 886ff.; intern war man sich zugleich bewusst, dass der Aufbau einer Verteidigungslinie am Elburs allein etwa $\$ 100$ Millionen an zusätzlichen Kosten für den Bau militärischer Anlagen mit sich brachte. Die JCS hatten das ElbursKonzept im April 1956 geprüft und nur im Kontext erheblicher Reformen der Kommandostruktur des Paktes und bei Zustimmung der Stationierung von Truppen der Allianzpartner auf verbündetem Territorium für einigermaßen realistisch eingeschätzt. Vgl. CONDIT, History of the JCS: VI, S. 161ff.; COHEN, Defending the Northern Tier, S. 191-7.

${ }^{195}$ Das State Department schlug zusätzliche Wirtschaftshilfe in Höhe von \$115 Millionen für die nächsten vier Jahre vor. Zugleich war man sich bewusst, dass es "little economic justification" für eine Fortsetzung der Wirtschaftshilfe gebe und die Motive vorwiegend politischer Natur seien. Vgl. die beiden Planning BoardDokumente auf S. 170, Anm. 192; Planning Board Briefing Paper for NSC, 31.1.57, ,Iran (8)', WHO, NSCS, Disaster File Ser., Box 66, DDEL; der Schah hatte bereits im Februar 1955 seinen Wunsch nach direkten Budgetzuschüssen in Höhe von etwa \$20 Millionen per Jahr geäussert, zweckgebunden an die Aufrüstung seiner Streitkräfte. Im Anschluss behandelte er den jährlichen Budgetzuschuss als amerikanische Gegenleistung für den iranischen Beitritt zum Bagdadpakt. Die Administration stimmte im Februar 1956 einer Fortsetzung der Budgethilfe zu - in Reaktion erhöhten die Iraner daraufhin ihr nächstes Budget rasch um weitere \$25 Millionen. Das Prochnow Committee hatte \$33 Millionen Budgetzuschuss für das Finanzjahr 1957 und insgesamt etwa \$70 Millionen für die folgenden drei Jahre empfohlen. Vgl. MemCon, 8.2.55; State \#536, 30.9.55; 276th NSC Meeting, 9.2.56; Allen Memo, 22.6.56, FRUS 55-57, XII, S. 709f., 776ff., 801-4, 820-8; vgl. Gustin Memo, 7.3.56, , OCB 091. Iran (File \#3) (7)', WHO, NSCS, OCB Central File Ser., Box 43, DDEL; mit einem Nuklearabkommen vom März 1957 und der anschließenden Lieferung von sechs Kilo hochangereicherten Urans und Vereinbarungen zum Bau eines kleinen Forschungsreaktors in Teheran wurde zudem das in späterer Zeit so kontroverse iranische Nuklearforschungsprogramm eingeleitet. Vgl. State \#1637, 1.3.57, CF 55-59, 611.8897/3-157; darüber hinaus gab es Unterstützung für zivile Nuklearprojekte im Rahmen der CENTO. Vgl. CENTO, Economic CommitteeScientific Council, Paper by Dr Smith, 6.5.61, ,CINS (CENTO Inst. of Nuclear Science), 1959-1960-1961', Lot 69 D 60-003, Office of International Scientific and Technological Affairs, CENTO Files, 1958-1966, Box 1, RG 59, NACP.

${ }_{196}$ Vgl. ed. note, FRUS 55-57, XII, S. 874.

197 Vgl. 334th NSC Meeting, 8.8.57, ebd., S. 934-7; Smith Draft, 9.8.57, ,334th Meeting', Official Meeting Minutes, Box 20, RG 273, NACP; der NSC hatte im Februar eine endgültige Entscheidung über die Höhe der Hilfsleistungen ausgesetzt, um die Auswirkungen der Joint Resolution 117, also der Eisenhower-Doktrin, miteinbeziehen zu können. Das House Committee on Government Operations veröffentlichte im Januar 1957 eine vernichtende Kritik des bisherigen Wirtschaftshilfeprogramms für Iran, das, so der Bericht, seit 1951 in etwa eine viertel Milliarde Dollar in ,a loose, slipshod, and unbusinesslike manner" verschwendet habe. U.S. HOUSE OF Representatives, Committee on Government Operations, United States Aid Operations in Iran; vgl. Bill, Eagle and Lion, S. $125 \mathrm{ff}$.
} 


\section{2. Intervention und Stabilität: Die USA und Iran zwischen Allianztreue und Reformbedarf, 1957-1960}

Hauptsächlich entscheidend für die praktisch unveränderte Fortsetzung der bisherigen Iranpolitik und die Ablehnung reformistischer Experimente war aber die veränderte Wahrnehmung der nahöstlichen Weltregion im Kontext der Suez-Krise. Wie oben geschildert, hatte die bedeutende amerikanische Rolle bei der Schaffung eines pro-westlichen Militärbündnisses in der Form des Bagdadpaktes und die grundsätzliche Unterstützung, trotz fortdauernder Reibungen zwischen London und Washington, der britischen Position zu einer eskalierenden Polarisierung der Region beigetragen. ${ }^{198}$ Dennoch reagierte die EisenhowerAdministration auf die panarabistische Rhetorik und die Annäherung Nassers an den Ostblock im März 1956 mit einer Neuorientierung der eigenen Nahostpolitik hin zu einer Eindämmungsstrategie gegen den radikalen arabischen Nationalismus nasseristischer Prägung. ${ }^{199}$ Die anglo-amerikanische Operation OMEGA startete im April 1956 mit dem Ziel, Nasser zu einer Aufgabe seiner neutralistischen und anti-westlichen Politik zu zwingen und zugleich die wahrgenommene Dominanz der ägyptischen Stellung im nahöstlichen Staatensystem zu beschneiden bzw. aufzuheben. Letzteres sollte unter anderem erreicht werden mittels einer stärkeren Unterstützung der pro-westlich orientierten Staaten in der Region, insbesondere auch des Bagdadpaktes, ,without actually adhering to the Pact or announcing our intention of doing so“. ${ }^{200}$ Die nun offen anti-ägyptische Positionierung Washingtons führte

\footnotetext{
${ }^{198}$ Im Januar 1956 hatten US-Offizielle in den anglo-amerikanischen Strategiegesprächen noch erhebliche Zweifel über den Sinn des Bagdadpaktes angemeldet. George V. Allen, ehemaliger US-Botschafter in Iran in den 1940ern, brüskierte die britischen Gesprächspartner mit der Feststellung: „If we decide that our Pact policy causes more damage than benefits, we will have to take another look at it.“; MemCon, 13.1.56, FRUS 55-57, XIII, S. 230; die geschichtswissenschaftliche Einschätzung der amerikanischen Rolle in der Entstehung einer kollektiven nahöstlichen Verteidigungsorganisation ist überwiegend negativ. PERSSON, Baghdad Pact, S. 328-37, interpretiert den Pakt aus der Perspektive anglo-amerikanischer Kooperation als grundsätzlichen Erfolg. Ähnlich das Fazit von COHEN, Defending the Northern Tier, S. 215-9; die Belastungen der special relationship infolge der Uneinigkeit über den Pakt betonen stärker OvEnDALE, Transfer of Power, S. 108-39; FREIBERGER, Dawn over Suez, S. 83106; TAKEYH, Eisenhower Doctrine, S. 48-72; und ASHTON, Hijacking, S. 135ff.; dagegen fokussieren Yeşilbursa, Baghdad Pact, S. 216-22; und DimitraKIS, Failed Alliances, S. 25-38, $187 \mathrm{ff}$.; auf das Scheitern der grundsätzlichen Intentionen hinter der Idee. Der negative Beitrag des Pakts zur intra-regionalen Polarisierung steht im Vordergrund bei PODEH, Quest for Hegemony, S. 243-52.

${ }_{199}$ Die Interpretation der älteren Forschungsliteratur, die Eisenhower-Administration hätte in antikommunistischer Übertreibung die nationalistischen Kräfte in der Region zuvorderst und fälschlich als Werkzeuge Moskaus wahrgenommen, kann als überholt gelten. Vgl. z.B. noch STIVERS, America's Confrontation, S. 4f.; ders., Eisenhower and the Middle East, S. 203-6; Zusammenfassung der historiographischen Korrektur in YAQUB, Containing Arab Nationalism, S. 2, 16-22, der die frühe Gegenposition bei BRANDS, Specter of Neutralism, S. 9f., im Kern bestätigt.

${ }^{200}$ Dulles Memo, 28.3.56, FRUS 55-57, XV, S. 420; vgl. British Government Paper, encl. to Makins Note, 21.3.56; NEA Memo, 28.3.56, ebd., S. 383-7, 409-18; vgl. Shuckburgh Minute, 10.3.56; Cabinet Conclusions, 21.3.56, BDEE A, 3, I, S. 500ff., 502f.; Eden Letter, 15.3.56, in: BOYLE, Eden-Eisenhower Correspondence, S. 123f.; vgl. auch die Version des Dulles Memo in DDRS: CK2349082553; vgl. YAQUB, Containing Arab Nationalism, S. 42ff.; ein US-Beitritt zum Bagdadpakt, so Dulles intern, würde eine Sicherheitsgarantie für Israel unumgänglich machen, was wiederum ,would quickly knock out Iraq“. MemCon, 28.3.56, FRUS 55-57, XV, S. 423; vgl. MemCon, 9.4.56, FRUS 55-57, XII, S. 275f.; zu OMEGA vgl. LuCAS, Divided We Stand, S. 93-134; LITTLE, American Orientalism, S. 169ff.; Eisenhower selbst bevorzugte die Loslösung Saudi-Arabiens von seiner ägyptischen Allianz und den Aufbau von König Sa' ūd zu einer alternativen Führungsfigur in der arabischen Welt.
} 
rasch zu einer Serie eskalierender Maßnahmen von beiden Seiten: Präsident Nasser beantwortete im Juli die Rücknahme der amerikanischen Zusage für finanzielle Unterstützung des geplanten Baus des Assuan-Staudammes mit der Verstaatlichung der Suezkanalgesellschaft. Die eben erst wiederhergestellte anglo-amerikanische Einheit in der Politik gegenüber Nassers Ägypten zerfiel nun an den unterschiedlichen Positionen in der Frage einer kriegerischen Lösung des Konflikts, was von amerikanischer Seite mit Blick auf die breiteren Sicherheitsinteressen im Kontext des globalen Kalten Krieges strikt abgelehnt wurde. $^{201}$ Im Gegensatz zu Attlees Nachgiebigkeit angesichts der amerikanischen Ablehnung von Gewalt in Ābādān 1951 war Premierminister Anthony Eden nicht bereit, britische Nahostinteressen ein weiteres Mal den Wünschen der amerikanischen Führungsmacht unterzuordnen. In Geheimgesprächen verabredeten sich Großbritannien, Frankreich und Israel zu einem Angriffskrieg gegen Ägypten, der mit einem Überraschungsangriff der Israelis auf den Sinai am 29. Oktober begann, gefolgt, getreu der Geheimabsprache von Sèvres der drei Aggressoren, von einem anglo-französischen Ultimatum und einer Militärintervention mit Landung von Truppenverbänden entlang der Kanalzone. Konfrontiert mit der Gefahr eines Konflikts mit der sowjetischen Supermacht, die ihrerseits in einem Ultimatum ein militärisches Eingreifen angedroht hatte, und besorgt über den vollständigen Zusammenbruch der westlichen Position im Nahen und Mittleren Osten, entschloss sich nun die Eisenhower-Administration zum Handeln gegen die strategischen Interessen ihrer europäischen Verbündeten - starker diplomatischer und vor allem wirtschaftlicher Druck zwangen London und Paris zur Aufgabe der Operation. Am 6. November schwiegen die Waffen. ${ }^{202}$

Verlauf und Folgen der Suezkrise bestätigten nachhaltig den bereits seit dem Zweiten Weltkrieg fortschreitenden Machtverlust der europäischen ehemaligen Kolonial- bzw. Mandatsmächte in

\footnotetext{
„We should be on a certain loser if we tried, as the Americans seem to be tempted to do, to build up King Saud as the leader of the Arab world", lautete dagegen die Einschätzung in London. Brook Memo, 14.4.56, PREM 11/1457, TNA:PRO; die Briten hatten mit stärkerer amerikanischer Unterstützung für den Bagdadpakt gerechnet ,[...] to transform it from a purely military alliance into an association which is demonstrably to the political, economic and social advantage of its members."; Memo for Cabinet Policy Review Committee, 1.6.56, BDEE A, 3, I, S. 510 .

${ }^{201}$ Eisenhower warnte Eden für den Fall einer kriegerischen Lösung, ,[...] the peoples of the Near East and of North Africa and, to some extent, of all of Asia and all of Africa, would be consolidated against the West to a degree which, I fear, could not be overcome in a generation and, perhaps, not even in a century particularly having in mind the capacity of the Russians to make mischief."; Eden hingegen warnte vor Nassers Ambition gerichtet auf die Schaffung eines „,united Arabia led by Egypt and under Russian influence“ mit Kontrolle sämtlicher nahöstlicher Ölvorkommen: „When that moment comes Nasser can deny oil to Western Europe and we here shall all be at his mercy."; Eisenhower Letter, 2.9.56; Eden Letter, 6.9.56, in: BOYLE, Eden-Eisenhower Correspondence, S. 163, 165; Eden erwähnte in dem Brief auch die Mahnung Mohammad Rezās, man müsse Nasser nun „loswerden“. Die Ablehnung einer gewaltsamen Lösung war eine Entscheidung der politischen Führung in Washington - die JCS empfahlen dagegen explizit das Erwägen einer militärischen Lösung, ,[...] to result in placing the Suez Canal under a friendly and responsible authority“; JCS Memo, 31.7.56, encl. to Robertson Memo, 2.8.56, FRUS 55-57, XVI, S. 117; die JCS bevorzugten allerdings eine anglo-französische Operation ohne direkte US-Beteiligung. außer im Falle eines sowjetischen Eingreifens. Vgl. CoNDIT, History of the JCS: VI, S. 180.

${ }^{202}$ In der Literatur zur Suez-Krise wird die bewusste Terminierung der Kriegshandlungen durch die Angreifer auf die Präsidentschaftswahlen in den USA und die offenkundige Kalkulation basierend auf dem Einfluss der ProIsrael-Lobby fast durchgehend ignoriert. Eisenhower schrieb im Nachgang einem Freund, er habe das State Department angewiesen , ,[...] that they should inform Israel that we would handle our affairs exactly as though we didn't have a Jew in America. The welfare and best interests of our own country were to be the sole criteria on which we operated."; Eisenhower Letter, 2.11.56, FRUS 55-57, XVI, S. 944.
} 
der Nahostregion. Britische Interessen und Institutionen wurden allerorts attackiert, der Einfluss Londons selbst auf die verbündeten Staaten Jordanien und Irak schwand sichtbar infolge der Demütigung britischer Macht. Eisenhowers instinktive Reaktion auf die „Schwächung der westlichen Position war, das entstandene „Vakuum“ zu füllen, „before it is filled by Russia.“203 Dem Präsidenten schwebte anfangs offenbar eine weitreichende amerikanische Hegemonierolle nach westeuropäischem Muster mit Sicherheitsgarantien und umfangreicher Wirtschaftshilfe vor, auch Nassers Ägypten einschließend. Die enorme Popularität der Vereinigten Staaten aufgrund ihrer als prinzipientreu anti-kolonial und proarabisch wahrgenommen Haltung in der Region während der Suezkrise war allerdings nur ein temporäres Phänomen. Bereits im Dezember setzte sich die unbestreitbare Priorität der transatlantischen Allianz durch und Washington näherte sich wieder Paris und London an. Der Eindruck amerikanischer Empfänglichkeit für innenpolitischen Druck durch die pro-IsraelLobby im Kontext des bis zum März 1957 verzögerten Rückzugs Israels aus dem Sinai und aus Gaza tat ein Übriges, das positive Bild der USA in der arabischen Welt zu überdecken. Entscheidend aber für die Konfrontation zwischen der westlichen Führungsmacht und dem radikalen arabischen Nationalismus unter Nasser war die Fortsetzung der amerikanischen Eindämmungspolitik gegen den ägyptischen Einfluss in der Region. Noch im November einigte man sich intern auf die Fortsetzung der OMEGA-Politik und den Aufbau regionaler Gegengewichte mit dem Ziel ,[...] to isolate Egypt and reduce Nasser's prestige and influence.“204 Frankreichs Rolle in der Region wurde infolge der Suez-Affäre als de facto beendet, Großbritanniens Position als ernsthaft beeinträchtigt angesehen: ,[...] the US must assume leadership in maintaining and restoring the Western position in the area.“205

Der neue amerikanische Führungswillen führte allerdings auch jetzt nicht - wie nun ein weiteres Mal seitens der JCS und des DOD, aber auch von den Mitgliedsstaaten gefordert - zu einem Beitritt der USA zum Bagdadpakt. ${ }^{206}$ Die Administration erklärte andererseits öffentlich ihre Unterstützung für den Pakt und gab eine, wenngleich noch vage, Sicherheitsgarantie für

\footnotetext{
${ }^{203}$ MemCon, 1.1.57, FRUS 55-57, XII, S. 433.

${ }^{204}$ Vgl. Eisenhower Memo for the Record, 8.11.56, FRUS 55-57, XVI, S. 1088f.; zum verzögerten Rückzug der Israelis aus dem Sinai vgl. HAHN, Caught, S. 210-20; Zitat aus State Outline Plan, encl. to Hoover Jr. Memo, 21.11.56, FRUS 55-57, XII, S. 350; fehlende Wörter bei YAQUB, Containing Arab Nationalism, S. 73; die frühen Pläne sahen nun sogar US-Unterstützung für panarabistische Föderationen im Maschrek und im Magreb unter Ausschluss Ägyptens vor. Zur alarmistischen Interpretation der JCS bez. Nassers regionalen Plänen vgl. JCS Memo, 3.8.56, encl. to Wilson Memo, 7.8.56, FRUS 55-57, XVI, S. 153-6; ähnlich sah man in London eine „growing resistance to Egyptian ambition“ in der Region und die Möglichkeit eines Zusammenschlusses der antinasseristischen Kräfte mit dem Ziel „to break up the Egyptian bloc"; Note by the Official Committee on the Middle East, 5.2.57, CAB 134/2339, TNA:PRO; vgl. GERGES, Superpowers and the Middle East; S. 79-99; MUFTI, Nasserist Pan-Arabism.

${ }^{205}$ Outline Plan, vorherig. Anm., S. 350; Eisenhower selbst kehrte zu seiner alten Präferenz zurück, König Sa ūd zu einer politischen Alternative aufzubauen. Er empfahl, die Schwächung Großbritanniens für eine pro-saudische Lösung der Buraimi-Frage zu nutzen. Vgl. MemCon, 21.11.56, FRUS 55-57, XII, S. 340ff.

${ }^{206}$ Vgl. CONDIT, History of the JCS: VI, S. 163f.; YeșilburSA, Baghdad Pact, S. 175-86; vgl. JCS Memo, 30.11.56; Wilson Letter, 4.12.56, FRUS 55-57, XII, S. 361ff., 372-6; auch Loy Henderson unterstützte den Beitritt. Siehe Henderson Memo, 6.12.56, ebd., S. 387ff.; zum Druck der Mitglieder vgl. Tehran \#731, 9.11.56; MemCon, 4.12.56; CIA, SNIE 40-7-56, 14.12.56, ebd., S. 318ff., 369-72, 401-9; Dulles hielt an den bisherigen Argumenten gegen einen US-Beitritt fest, spielte aber nun mit dem alternativen Gedanken eines iranischen Beitritts zur SEATO. Im Gegensatz zum Bagdadpakt waren die USA SEATO-Mitgliedsstaat. Vgl. Dulles Memo, 16.11.56, ebd., S. 330f.; zur britischen Haltung vgl. No. 277 from Wright, 22.11.56, BDEE B, 4, III, S. 536ff.
} 
dessen Mitglieder ab. ${ }^{207}$ Letztlich entschied sich die Eisenhower-Administration für eine in erster Linie bilaterale Herangehensweise mit dem Ziel der Aufwertung der eigenen Position in der Region, wohl auch den vielen negativen Erfahrungen der vorhergehenden Versuche zur Organisation kollektiver Verteidigungsorganisationen geschuldet. Der Bilateralismus mündete schließlich in die Eisenhower-Doktrin, verkündet vor dem Kongress am 5. Januar 1957. In seiner Rede ersuchte der Präsident den US-Kongress um eine Resolution zugunsten einer Erhöhung der Wirtschafts- und Militärhilfe für die nahöstlichen Staaten sowie, der wichtigste Bestandteil, um eine vorbeugende Autorisierung zum Einsatz militärischer Mittel ,[...] to secure and protect the territorial integrity and political independence of such nations [...] against overt armed aggression from any nation controlled by International Communism.“208 Die Betonung der anti-kommunistischen Zielsetzung der Doktrin war in Teilen innenpolitischen Erfordernissen geschuldet, wobei intern (und auch in Kairo) keine Zweifel bestanden, dass sich das verstärkte amerikanischen Engagement in der Region auch und gerade gegen ägyptische Dominanzbestrebungen und den radikalen arabischen Nationalismus richtete. ${ }^{209}$ Nasser nannte die Doktrin Jahre später ein ,device to re-establish imperial control by non-military means“ und ,[...] directed at Egypt as much as at any communist threat“". ${ }^{210}$

Zur Festlegung und Verteilung der in der Eisenhower-Doktrin erwähnten zusätzlichen Wirtschafts- und Militärhilfe für die pro-westlichen Staaten in der Region - anfangs war von durchaus beachtlichen zusätzlichen $\$ 400$ Millionen für die nächsten zwei Jahre die Rede - hatte der Präsident den ehemaligen demokratischen Kongressabgeordneten James P. Richards zum Sonderbotschafter ernannt. Die Richards-Mission in verschiedene Staaten in der Region zwischen März und Mai 1957 erreichte die Unterstützung der Doktrin durch eine Reihe prowestlicher Staaten, darunter der Irak, Tunesien, Libyen und der Libanon. ${ }^{211}$ Zugleich machte Richards im Namen der Administration eine Reihe von Zusagen über zusätzliche Unterstützungsleistungen, allerdings in weitaus geringerem Rahmen als erwartet, was mit einiger Enttäuschung aufgenommen wurde. Anfängliche Erfolge im Sinne der anti-

\footnotetext{
${ }^{207}$ Siehe ed. note, FRUS 55-57, XII, S. 360; intern gab es - letztlich aufgegebene - Vorschläge zur Gründung einer neuen und unbelasteten regionalen Gruppierung, wieder gegen Ägypten gerichtet, in die der Bagdadpakt aufgehen würde. Vgl. Rountree Memo, 5.12.56, ebd., S. 376-82; auch die Briten spekulierten zwischenzeitig über die Vorzüge einer Art „super NATO“ unter Einschluss der Bagdadpaktstaaten. Provisorisch müssten die Amerikaner aber ,[...] 'temporarily' replace the British influence in the Baghdad Pact, 'where, at least for the present, we are somewhat 'smelly'."; MemCon, 4.12.56, ,Hanes-Macomber [...] (2)', JFDP, Special Assistants Chronological Ser., Box 11, DDEL.

${ }^{208}$ Special Message to the Congress, 5.1.57, PPP: Eisenhower 1957, S. 13; die Bevorzugung des bilateralen Ansatzes nachvollziehbar in MemCon, 8.12.56, FRUS 55-57, XII, S. 396f.; Repräsentantenhaus und Senat verabschiedeten die entsprechende Joint Congressional Resolution im Januar bzw. im März. Vgl. ed. note, ebd., S. 452.

${ }^{209}$ Vgl. Murphy Memo, 15.12.56, ebd., S. 410ff.; innerhalb der Administration vermengten sich anti-ägyptische und anti-sowjetische Motive. Vgl. CIA, SNIE 11-10-56, 29.11.56, ebd., S. 355-60; zu Nassers Reaktion vgl. Cairo \#2222, 10.1.57, FRUS 55-57, XVII, S. 16ff.; zur Formulierung der Eisenhower-Doktrin vgl. vor allem YAQUB, Containing Arab Nationalism, S. 68-85; auch TAKEYH, Eisenhower Doctrine, S. 142-53; HAHN, Eisenhower Doctrine, S. 38-40; DAMMS, American Strategy, S. 179-82.

${ }^{210}$ Cairo \#861, 27.5.60, FRUS 58-60, XIII, S. 584.

211 Zudem hatten die nichtarabischen Mitglieder des Bagdadpaktes sowie Äthiopien Zustimmung signalisiert. Saudi-Arabien nahm zwar zusätzliche Wirtschaftshilfe über \$20 Millionen an, vermied aber eine unzweideutige Akzeptanz der Doktrin.
} 
nasseristischen Zielsetzung hinter den neuen US-Politik waren die Ausschaltung der nationalistischen Opposition in Jordanien im Frühling 1957 durch König Hussein, die dabei geleistete Unterstützung seitens Saudi-Arabiens und Iraks, in deren Zuge sich auch die saudischen und haschemitischen Dynastien wieder annäherten, und die Konsolidierung der Machtposition des pro-westlichen, libanesischen Präsidenten Camille Chamoun. ${ }^{212} \mathrm{Ab}$ dem Sommer 1957 zeigten sich aber die Fehlannahmen hinter der Doktrin allzu deutlich. Der gescheiterte Versuch der USA und Großbritanniens mittels ,covert action“ den Drift der syrischen Politik nach links umzukehren, fachte ein weiteres Mal die Polarisierung in der Region an. ${ }^{213}$ Zudem führte die aggressive Politik im Kontext der Anwendung der EisenhowerDoktrin gegen Syrien zu einer - wenngleich in der Literatur des Kalten Krieges meist ignorierten - gravierenden Krise in den Beziehungen zwischen Washington und Moskau inklusive einer temporären Gefahr eines Ausbruchs kriegerischer Auseinandersetzungen zwischen den Supermächten. Sowohl während der Suezkrise von 1956 als auch in den Krisen in Jordanien und Syrien im April und August 1957 wurde die Sechste US-Flotte ins östliche Mittelmeer verlegt und eine Reihe weiterer militärischer Vorbereitungsmaßnahmen umgesetzt. So wurde sogar zwei Mal die Alarmbereitschaft des Strategic Air Command heraufgesetzt, dabei eine nukleare Konfrontation zumindest in Kauf nehmend. ${ }^{214}$ Letztlich war es die auf Beruhigung der Lage abzielende saudische und irakische Diplomatie, welche die syrische Krise entschärfte und eine durchaus denkbare Konfrontation zwischen den Supermächten im Nahen Osten zu vermeiden half. ${ }^{215}$

Die Syrienkrise von 1957 demonstrierte eigentlich augenfällig die konzeptionellen Fehlannahmen hinter der Politik der Eisenhower-Doktrin. Angesichts des fortgesetzten „Arab Cold War" (Malcolm Kerr), der kulturellen Hegemonie anti-imperialistischer und panarabistischer Ideen in breiten Bevölkerungsschichten, und der, grundsätzlich anders als in Westeuropa, kaum vorhandenen Perzeption einer Bedrohung seitens der Sowjetunion war die Durchsetzung einer umfassenden amerikanischen Hegemonie inklusive einer Steuerungs- und Ordnungsfunktion gegenüber der regionalen Staatenwelt nie auch nur in Ansätzen realistisch gewesen. Eine neue NSC-Studie zur breiteren amerikanischen Politik in der Region im Januar 1958 verwies bereits darauf, dass das bisherige Ziel Washingtons ,to organize the entire area for defense against Communist aggression and subversion“ angesichts der Haltung der

\footnotetext{
${ }^{212}$ Vgl. YAQUB, Containing Arab Nationalism, S. 120-45.

${ }^{213}$ Die bereits oben erwähnte, insgesamt als fahrlässig bis verantwortungslos zu bewertende Operation WAPPEN mit dem Ziel des Sturzes der linksgerichteten syrischen Regierung musste unmittelbar nach der scharfen Kritik an der „King Making“-Attitüde der CIA im Bruce-Lovett wie eine nachträgliche Bestätigung wirken. Vgl. ebd., S. 147-80; man müsse einen Vorwand für eine Intervention finden, so Macmillan in seinem Tagebuch. Der ,,mantle of Anthony Eden“ sei nun auf Foster Dulles gefallen. Eintrag vom 22.9.57, in: CATTERALL, Macmillan Diaries: I, S. 60; auf die Aufdeckung des geplanten Staatsstreichs reagierte die Eisenhower-Administration mit Planungen, eine Invasion Syriens durch die Nachbarstaaten zu provozieren. Vgl. LiTTLE, Mission Impossible, S. 676f.; Rathmell, Covert Struggle, S. 125-44; JONES, Preferred Plan.

${ }^{214}$ Vgl. CONDIT, History of the JCS: VI, S. 178-92; HAHn, Caught, S. 206f., 236; ders., Eisenhower Doctrine, S. 43.

${ }^{215}$ Nasser selbst hatte im Oktober 1957 für alle Akteure überraschend eine symbolische Truppenpräsenz in Syrien stationiert und das Land somit vor einer Invasion geschützt sowie seinen regionalen Führungsanspruch zur Schau gestellt.
} 
öffentlichen Meinungen im Nahen und Mittleren Osten auf absehbare Zeit hin unerreichbar sein werde. $^{216}$ Zwar hielt man zu diesem Zeitpunkt noch an der anti-nasseristischen Stoßrichtung fest, doch akzeptierte man zugleich die Existenz neutralistisch orientierter Staaten und setzte zudem wieder verstärkt auf Zusammenarbeit mit den revolutionären und nationalistischen Kräften und versuchte bisweilen diese zu kooptieren, sofern sie nicht pro-sowjetisch orientiert waren. So gestand man sich zögerlich ein, dass die im Februar 1958 erfolgte Vereinigung Ägyptens und Syriens zur Vereinigten Arabischen Republik (VAR) die Sorgen über eine kommunistische Machtübernahme in Damaskus beseitigt hatte. Dennoch blieben die antinasseristischen Reflexe innerhalb der Administration weiterhin stark: „Radical nationalism has shown itself entirely willing to facilitate the penetration of the Near East by international Communism and openly proclaims its desire to eradicate Western positions and influence from the area. ${ }^{\circ 217}$

Unverändert blieb aber der amerikanische Führungsanspruch: die USA trügen nun die „,major responsibility“ als westliche Vormacht in der Nahostregion. ${ }^{218}$ Damit einher ging eine weitere Abwertung der britischen Position in der nahöstlichen Politik aus amerikanischer Sicht. Zwar hatte sich die Eisenhower-Administration unmittelbar nach der Suezaffäre um eine gewisse Rehabilitierung der britischen Macht im Nahen Osten bemüht und das Treffen zwischen Eisenhower und Harold Macmillan - der Schatzkanzler hatte im Januar den diskreditierten Eden als Premierminister abgelöst - auf den Bermuda-Inseln im März 1957 reanimierte die bereits tot geglaubte special relationship zwischen den anglo-amerikanischen Mächten. Eisenhower versicherte dabei den Briten, ,[...] that the US wants [...] to build them up again in the Middle East.“219 Auf britischer Seite war man sich der geänderten Lage schmerzlich bewusst. Unmittelbar nach der Suez-Affäre hatte Macmillan in einem vertraulichen Gespräch mit dem US-Präsidenten die eigene gescheiterte Politik gegenüber Nassers Ägypten als den „[...] last gasp of a declining power“ bezeichnet. ${ }^{220}$ Das Official Committee on the Middle East des Kabinetts hatte bereits Ende 1956 grundsätzlich eingestanden, dass es nunmehr keine Alternative gebe zu einer Politik , in the area in step with that of the United States““. ${ }^{221}$ Damit

\footnotetext{
${ }^{216}$ Rountree Memo, 21.1.58, FRUS 58-60, XII, S. 5; NSC 5801/1 ,Long-Range U.S. Policy Toward the Near East‘, encl. to Lay Note, 24.1.58, ebd., S. 17-32; NSC 5801/1 ersetzte die oben erwähnte Studie NSC 5428 vom Juli 1954. Zum geflügelten Begriff des Arab Cold War siehe den locus classicus von KerR, Arab Cold War; MUFTI, Sovereign Creations, S. 65-81; insgesamt zur US-Politik in der Region nach Suez vgl. DAMMS, American Strategy; SCHULZINGER, Impact of Suez; KUNZ, Emergence.

${ }^{217}$ State Assessment, encl. to Rountree Memo, 24.3.58, FRUS 58-60, XII, S. 54.

${ }^{218}$ NSC 5801/1, Anm. 216, hier S. 24f.

${ }_{219}$ MemCon, 21.3.57, FRUS 55-57, XXVII, S. 716; vgl. ASHTON, Golden Days, S. 698ff.; ders., Problem of Nasser, S. 114-21; zum britischen Ansatz gegenüber Washington vgl. RUANE/ELLISON, Power-by-Proxy; YAQUB, Containing Arab Nationalism, S. $112 \mathrm{ff}$.

${ }^{220}$ Macmillan fuhr fort: ,,[...] perhaps in two hundred years the United States 'would know how we felt' "“; Memo for the Record, 12.12.56, ,Memos [...] L Through M (2)‘, JFDP, General Correspondence and Memoranda Ser., Box 1, DDEL; in taktischer Hinsicht hoffte man aber, die Amerikaner von ihrer negativen Haltung gegenüber dem britischen Kolonialismus abzubringen. Vgl. Macmillan Reply to Lord Perth und folgende minutes, 25.2.57, BDEE A, 4, II, S. 228ff.

${ }_{221}$ M.E. (O)(56)80, 7.12.56; CAB 21/3934, TNA:PRO; „Consequently it is not realistic to plan in terms of local wars in the Middle East save those fought with the United States as our active ally or at least a benevolent neutral. [...] We must deploy what efforts we can to encourage them to accept responsibilities in the Middle East and to
} 
war aber keineswegs eine Unterordnung der eigenen Position gemäß amerikanischen Wünschen gemeint, sondern die aktive Beeinflussung der Politik der EisenhowerAdministration zum Vorteil der verbliebenen britischen Einflussbereiche, zuvorderst der britischen Vormachtstellung im ölreichen Persischen Golf und im Irak. Trotz des SuezDebakels hielt die Macmillan-Regierung entschlossen an der Bewahrung der Überbleibsel des Empire in der Nahostregion fest. Die verbliebene Position in Südarabien und im Golf wurde nun als ,integrated whole“ konzipiert, wonach ein Zurückweichen an einer Stelle zwangsläufig den Zusammenbruch der gesamten britischen Stellung nach sich ziehen würde, eine Konstruktion, die nicht zuletzt gegenüber den nun dominanten Amerikanern die Unmöglichkeit britischer Zugeständnisse gegenüber dem sich weiter ausbreitenden arabischen Nationalismus vermitteln sollte. $^{222}$ Macmillan betonte im Kabinett, dass es wichtig sei ,[...] in present circumstances we should not create the impression of withdrawing entirely from the Middle East. “223

Tatsächlich unterstützte die Eisenhower-Administration im Grundsatz die Fortsetzung der britischen Rolle im Persischen Golf und schien sogar zeitweilig den britischen Wünschen nach vertiefter und institutionalisierter anglo-amerikanischer Kooperation in der Nahostregion entgegenzukommen. ${ }^{224}$ Eine während der Syrienkrise von 1957 eingerichtete streng geheime Syria Working Group diente im Anschluss an die Washingtoner Gespräche vom Oktober 1957 als Modell für die Einberufung einer ganzen Reihe anglo-amerikanischer „Arbeitsgruppen“, um die Außenpolitik der beiden Verbündeten in den verschiedensten Bereichen enger zu koordinieren und zu planen. Premier Macmillan pries die Vereinbarung als eine neuartige „Declaration of Inter-Dependence“ und betrachtete sie als programmatischen Ausdruck einer geteilten anglo-amerikanischen Führungsrolle innerhalb der westlichen Allianz. Macmillan

seek irrevocably to commit them to the major role in the defence of common positions which we have hitherto sustained."; Nutting Memo, 26.12.56, ebd.

${ }_{222}$ Vgl. O.M.E.(57)14(Revise) Addendum ,Anglo-American Co-operation in the Middle East', 13.3.57, CAB $134 / 2339 ;,[\ldots]$ no part of which can be weakened or resigned without affecting the rest. [...] We should therefore as far as possible make no concessions which would jeopardise the territorial integrity of the Persian Gulf States and we should continue to protect them against any encroachment by their more powerful neighbours, particularly Saudi Arabia.“; Selwyn Lloyd Memo, C.(57)138, 7.6.57, CAB 129/87, fol. 215-223; die Enttäuschung der USAdministration über die mangelnde Kompromissbereitschaft Londons bez. der Buraimi-Frage war in den Gesprächen allgegenwärtig. Suez hatte zudem die Bedeutung des Bagdadpaktes aus britischer Sicht noch weiter aufgewertet: ,The Pact is now more than ever the lynch-pin of our position in the Middle East, including Pakistan [...].“; O.M.E.(57)11(First Revise), 11.3.57, CAB 134/2339, TNA:PRO; ein US-Offizieller empfand die britische Neueinschätzung der eigenen Rolle im Golf als ,,more rigid than in the past“ und die Idee eines ,,integrated whole“ als „,nonsense“. Mathews Memo, 13.5.57, ,General/British Position [...]", Lot 61 D 48, Records Relating to the Arabian Peninsula, 1948-1959, NEA/NE, OiCIA, Arabian Peninsula Affairs, Box 15.

${ }^{223}$ C.C.(57)1, fol. 24, 15.1.57, CAB 128/31, TNA:PRO; somit erzeugte Suez letztlich nur einen begrenzten und qualifizierten britischen Rückzug aus der Nahostregion. Zum Forschungsstand vgl. SMITH, Power Transferred?; ders., Aftermath of the Suez Crisis; BlaCKWEll, Transfer of Power?; Elliot, Defeat and Revival.

${ }^{224}$ So empfahl Eisenhower selbst in Bermuda den Briten, ,[...] all aspects of policy in the area from the point of view of holding Kuwait and its oil" zu betrachten. Das Zitat entstammt allerdings der britischen Aufzeichnung des Gesprächs. Siehe Bermuda Conference 2nd Meeting, 21.3.57, PREM 11/1838, TNA:PRO; zu Eisenhowers eigener Absicht in diesem Kontext vgl. seinen diary entry vom 21.3.57, FRUS 55-57, XXVII, S. 718-21; die in Bermuda vereinbarten Gespräche über die Sicherung des Zugangs zu den nahöstlichen Ölquellen führten zu der Übereinkunft, dass beide Staaten, should cooperate to preserve beneficial U.K. relationships with the Persian Gulf and Arabian See principalities of special importance to the supply of oil to the free world, including especially Kuwait, Bahrain, Qatar, Sharja and Muscat."; Agreed Paper, encl. to Henderson Memo, 20.6.57, FRUS 55-57, XII, S. 548-53; Memo for Macmillan, 28.6.57; Dulles Letter, 24.6.57, PREM 11/1937, TNA:PRO; Bishop Minute, 8.6.59, BDEE A, 4, I, S. 243 f. 
selbst hatte im Zweiten Weltkrieg die Analogie der britischen „Griechen“ im „römischen Imperium“ der Amerikaner eingeführt: man solle die US Politik inspirierend anleiten, ähnlich wie ,the Greek slaves ran the operations of Emperor Claudius“. ${ }^{225}$ Im Kabinett in London feierte man die amerikanische Übernahme des britischen strategischen Konzeptes der „,interdependence“ mittels der ,working group machinery“: eine übereinstimmende angloamerikanische Nahostpolitik sei endlich denkbar. ${ }^{226}$ Was auf den ersten Blick wie eine Erfüllung des alten Traums Winston Churchills hinsichtlich einer Neubelebung der angloamerikanischen Weltkriegsallianz inklusiver integrierter Militärplanung erschien, entpuppte sich rasch als nur begrenzt funktionierender und zudem von amerikanischer Seite nur halbherzig betriebener Konsultations- und Koordinierungsapparat. ${ }^{227}$ Letztlich stellte allein der Eindruck der Existenz einer exklusiven anglo-amerikanischen Planungsgruppe eine Belastung für die anderen Bündnisbeziehungen Washingtons dar. Mit Bezug auf die Nahostregion zeigten sich hier zudem ein weiteres Mal die grundsätzlichen Differenzen zwischen britischer und amerikanischer Herangehensweise, gerade was die Prioritätensetzung hinsichtlich der kommunistischen gegenüber der nasseristischen Gefahr betraf. ${ }^{228}$ Der stattfindende Austausch in der bisherigen Arbeitsgruppe zu Syrien, kurz darauf umbenannt in die Middle East Working Group, unter Beteiligung von CIA und MI6, führte offenkundig auf amerikanischer Seite zu einiger Ernüchterung sowohl über britische Absichten, Methoden wie auch tatsächlich verfügbare Kapazitäten, welche die von London empfohlene Strategie eines „Containment $P l u s$ “ in der nahöstlichen Weltregion leiten und unterfüttern sollten. ${ }^{229}$

${ }^{225}$ Zit. aus Ashton, Golden Days, S. 697; Macmillan Report to the Cabinet, 28.10.57, BDEE A, 4, II, S. 237ff.; siehe auch den kuriosen Eintrag in Macmillans Tagebuch am 23.8.57: ,[...] I suggested that Syria may be the Czechoslovakia we need to make a NATO for the Middle East."; in: CATTERALL, Macmillan Diaries: II, S. 55. ${ }^{226}$ See C.C. (57) 76, 28 Oct. 1957, CAB 128/31; Lloyd Note, 10.4.58, CAB 129/92, TNA:PRO.

${ }^{227}$ Die Existenz der working groups war streng geheim - das britische Kabinett selbst wurde erst im April 1958 darüber informiert. Siehe MemCon, 23.10.57, DDRS: CK2349322048; teilweise auch in FRUS 55-57, XXVII, S. 807-12; FO Brief Serial N5, 4.6.58, CAB 130/147, TNA:PRO; vgl. BLACKWELL, Struggle for Jordan, S. 89-94; Ashton, Problem of Nasser, S. 134-9; ders., Golden Days, S. 699-706; grundlegend JoNES, Working Group Experiment; ein ehemaliger NEA-Offizieller behauptete später, die Vereinbarungen einer engen angloamerikanischen Kooperation seien von US-Seite nur aus psychologischen Gründen akzeptiert worden: ,In other words there were things we were doing with them simply to have them think that Philby and the Suez Canal crisis hadn't had all that much effect on our relationships."; Harrison M. Symmes OHI, 25.2.89, FAOH.

${ }^{228}$ Vgl. AsHTON, Problem of Nasser, S. 140-9; BLACKWELl, Pursuing Nasser, S. 88-91; weiterhin belastend blieb zudem die Buraimi-Frage. In London war man ernüchtert über die amerikanische „Obsession“ bez. britischer Kompromissbereitschaft. Man müsse daher den Amerikanern klar machen, dass „,[...] the fulfilment of our obligations to the Ruler of Abu Dhabi and the Sultan of Muscat \& Oman in respect of the oasis is the touchstone of our ability and willingness to protect the northern Persian Gulf states which are of major strategic and economic importance to the Western position in the Middle East."; andernfalls würden sich diese Herrscher Nasser zuwenden, so die wenig überzeugende Argumentation. V1075/18, D.M.H. Riches Minute, 8.4.57, FO 371/127756, TNA:PRO.

${ }^{229}$ Bereits in den Gesprächen vom Sommer 1957 tat sich die britische Seite mit einem Vorschlag zur Ermordung von Teilen der syrischen Staatsführung hervor. Vgl. JONES, Preferred Plan, S. 408f.; tatsächlich war man gerade auf Seiten der Nahostexperten beider Seiten, darunter wieder einmal Kermit Roosevelt, in der Working Group eher skeptisch, was die generelle anti-nasseristische Stoßrichtung anging. So empfahl die Gruppe bereits im Oktober ,an examination of the question of whether an easing of relations with Egypt would be likely to improve the US and UK position in the Middle East and advance Western policy objectives with respect to Syria."; zugleich kritisierte man das Ignorieren des ,primary cause“ der westlichen Unterstützung für Israel für die sich verschlechternden Beziehungen zur arabischen Welt und empfahl eine grundlegende Kehrtwende, u.a. ,a concerted effort to educate public and government opinion in the US and the UK on the real facts of the Middle East situation, with particular emphasis on the disastrous implications to the position of the West of continuing substantially unchanged our existing policies toward Israel."; Morris Letter mit enclosures, 11.10.57, PREM 11/2521, TNA:PRO; stattdessen wurde die Propaganda gegen Nasser wieder verschärft. Vgl. VAUGHAN, Failure of Propaganda, S. 229-37; in der CIA hatte man sich bereits im Zuge von TPAJAX über die begrenzten Kapazitäten des SIS in der Region gewundert. So habe es z.B. keinen ausschließlich für Iran zuständigen Analysten 
Intern wurden Stimmen in der Eisenhower-Administration laut, die eine Aufgabe der „,interdependence“ anrieten. Ein NSC-Papier vom Januar 1958 stellte nüchtern fest, dass amerikanische Zielsetzungen ,with respect to the British in the area will no longer revolve around the usefulness per $\underline{s e}$ of the maintenance of a British position in the area", sondern vielmehr ,by our very real interest in maintaining Britain as a strong and prosperous member of the Western alliance.“230 Eine konsequente Anpassung der eigenen Nahostpolitik an diese Erkenntnis unterblieb aber vorerst aufgrund der Volatilität der Lage in der Region. Die irakische Revolution vom Juli 1958 und der Sturz der haschemitischen Dynastie in Bagdad und damit der Verlust des einzigen arabischen Mitgliedsstaats des Bagdadpaktes erschütterten ein weiteres Mal die westliche Position in der Nahostregion. Der befürchtete Domino-Effekt infolge der Ereignisse von Bagdad, der Sturz der verbliebenen pro-westlichen und konservativen Regierungen, erzeugte in Washington kurzzeitig eine apokalyptische Stimmung. John Foster Dulles sah die gesamte Region in Gefahr einer kommunistischen Übernahme - für ihn gleichbedeutend mit der nasseristischen Bedrohung - und empfahl die Errichtung von ,sand bags around positions we must protect", zuvorderst Israel, der Libanon, und die Ölquellen des Persischen Golfs. Trotz der expliziten Skepsis des Präsidenten über die Durchsetzbarkeit einer militärischen Lösung setzten sich die Interventionisten vorerst durch. ${ }^{231}$ Was folgte waren die - parallelen und eben nicht gemeinsamen (Stephen Blackwell) - amerikanischen und britischen Militärinterventionen im Libanon und in Jordanien mit dem Ziel der Stabilisierung der prowestlichen Führungen in diesen Staaten und der, wenngleich weitgehend sinnlosen, Entsendung eines Bataillons von US-Marines in den Golf. ${ }^{232}$

Trotz seiner Zustimmung zum militärischen Eingreifen in der Nahostregion verweigerte sich Eisenhower weitreichenden Plänen wie dem von Macmillan gewünschten ,showdown“ mit dem radikalen arabischen Nationalismus und Präsident Nasser mit dem Ziel des „,clearing up the whole mid-East situation.“233 In der Retrospektive war die Libanonintervention von 1958

im Hauptquartier der britischen Kollegen gegeben, berichtete Wilber in seiner internen Geschichte. Vgl. CIA [Donald N. Wilber], Clandestine Service History, S. 87.

${ }^{230}$ Draft NSC Staff Study on NSC 5801, 16.1.58, DDRS: CK2349454872; Macmillan hingegen hatte auf eine Rückkehr der prä-Suez-Zusammenarbeit gehofft. ,The Americans, of course, are now completely converted - too late - and wish devoutly that they had let us go on and finish off Nasser."; Eintrag vom 19.12.57, in: CATTERALL, Macmillan Diaries: 1950-1957, S. 78; vgl. FO Note for Future Policy Study Working Group, 6.10.59, BDEE A, 4, I, S. 71-9.

${ }^{231}$ MemCon, 23.7.58, FRUS 58-60, XII, S. 98, Eisenhower warnte explizit vor rein militärischen Lösungen: „Even if we put in large military forces we cannot see what to do beyond that point."; MemCon, 20.7.58, ebd., S. 84; allerdings teilte Eisenhower die Einschätzung Dulles' zu Nasser als de-facto-Unterstützer Moskaus. Er sei eine sowjetische Marionette, ,even though he probably doesn't think so.“; MemCon, 15.7.58, FRUS 58-60, XI, S. 245. ${ }_{232}$ Vgl. Blackwell, Transfer of Power?, S. 175; zur nationalistischen Revolution im Irak und zur angloamerikanischen Reaktion vgl. ASHTON Response; BLACKWELL, Desert Squall; zu den US-Motiven vgl. GENDZIER, United States Intervention; zu den langfristigen Effekten des Eingreifens auf die Staatsbildungsprozesse in der Region vgl. SCHAYEGH, 1958 Reconsidered.

${ }^{233}$ Vgl. Eisenhower Letter, 18.7.58; TelCon, 14.7.58; MemCon, 15.7.58, FRUS 58-60, XI, S. 330, 232, 245; Washington Embassy to Macmillan, 19.7.58, CAB 21/3252; allerdings bekräftigte Eisenhower in seinem Schreiben an Macmillan die Notwendigkeit ,to [...] see that the Persian Gulf area stays within the Western orbit. The Kuwait - Dhahran - Abadan areas become extremely important and Turkey and Iran have become more important."; die Überbleibsel der alten Suez-Gruppe argumentierten weiterhin für einen aggressiven Kurs in London. Die Logik hinter der „Suez expedition still holds good“ und daher müsse es das Ziel sein Nasser zu 
nicht der Höhepunkt in der Umsetzung der Eisenhower-Doktrin, sondern Auslöser ihrer letztendlichen Aufgabe. Wenngleich militärisch erfolgreich, da praktisch auf keinerlei Gegenwehr treffend, demonstrierten die Interventionen nach Ansicht Eisenhowers dennoch die Unzulänglichkeit rein militärischer Maßnahmen: „[...] a Western position cannot be held against the underlying and often unthinking convictions of the Arab world."234 Angesichts der Perzeption in Washington über eine zunehmende kommunistische Gefahr in der Region wandelte sich alsbald die Einschätzung gegenüber Nasser. Eine Einschätzung des NSC Planning Board sprach die Hoffnung aus, der arabische Nationalismus ,[...] may prove to be the greatest counter-force to Soviet penetration“. ${ }^{235}$ Eine neue NSC Studie zur amerikanischen Nahoststrategie, NSC 5820/1 vom Oktober 1958, trug dieser neuen Einsicht Rechnung. Statt den Nasserismus weiter zu bekämpfen und die arabischen Nationalisten in die Arme Moskaus zu treiben, erstrebte man nun die Etablierung einer ,working relationship“ mit der politisch wie ideologisch dominanten Kraft in der Region. Wesentliche Bestandteile der EisenhowerDoktrin selbst seien nunmehr als ,out of date“ anzusehen. ${ }^{236}$

Es folgte im Anschluss eine stetige Verbesserung der Beziehungen zwischen Kairo und Washington, inklusive einer, wenngleich begrenzten, Zusammenarbeit gegen ein kommunistisches Vordringen im post-revolutionären Irak sowie im Imamat des Nord-Jemen. ${ }^{237}$ Nasser selbst ging scharf gegen kommunistische Kräfte in Ägypten und in der VAR-Provinz Syrien vor und wurde dafür alsbald mit generösen Zusagen amerikanischer Nahrungsmittelhilfe im Rahmen des PL 480 (Food for Peace)-Programms belohnt. ${ }^{238}$ Das Rapprochement mit Kairo Ende der 1950er Jahre löste zumindest vorübergehend das größte Problem der amerikanischen Nahoststrategie, führte aber zugleich zu Belastungen im Verhältnis zu Großbritannien und den pro-westlichen Staaten in der Region, zuvorderst mit Israel. Die Israelis reagierten auf die amerikanisch-ägyptische Kooperation mit einer Intensivierung ihrer

„zerstören“, „,if he pursues his career of aggression against our friends.“; Lennox-Boyd Memo, M.E.(M)(59)4, 4.2.59, CAB 134/2230; Amery Letter, 8.9.58, PREM 11/2397, TNA:PRO; vgl. auch Templer Memo, 11.8.58, BDEE A, 4, I, S. $237 \mathrm{ff}$.

${ }^{234}$ MemCon, 23.7.58, FRUS 58-60, XII, S. 93; in einem Entwurf eines Schreibens an Macmillan hatte Eisenhower als eigentliche politische Kernfrage im Nahen Osten beschrieben ,whether or not the Western world can maintain its rightful opportunity to purchase vitally needed oil supplies peaceably and without hindrance or payment of blackmail.“; Unused Draft, 15.7.58, ,DDE Dictation - July 1958“, AWF, DDE Diary Ser., Box 34, DDEL.

${ }^{235}$ NSC Planning Board Paper, 29.7.58, FRUS 58-60, XII, S. 117; Eisenhower selbst schien dem zuzustimmen: ,Since we are about to get thrown out of the area, we might as well believe in Arab nationalism."; 374 th NSC Meeting, 31.7.58, ebd., S. 132; zur Verbindung von Ölinteressen und Haltung gegenüber dem Nasserismus vgl. insbesondere NSC Planning Board Paper, 29.7.58, ebd., S. 114-24.

${ }^{236}$ Annex A to NSC 5820 Draft Paper, 3.10.58, DDRS: CK2349245960, S. 52; für die endgültige Version der NSC-Studie 5820/1 siehe ,Statement of U.S. Policy Toward the Near East', encl. to NSC Report, 4.11.58, FRUS 58-60, XII, S. 187-99; zur Erklärung des zugrunde liegenden Politikwandels vgl. Rountree Memo, 10.10.58, ebd., S. 167-70; zur Formulierung von NSC 5820/1 vgl. POPP, Working Relationship; YAQUB, Containing Arab Nationalism, S. 237-67; DAMMS, American Strategy; S. 186-92; grundlegend zum Wechselspiel zwischen regionalen und globalen Politikzielen KuPCHAN, Roots of Regional Security Policy.

${ }^{237}$ Vgl. PoPP, Working Relationship; CiTINO, Middle East Cold Wars; die britische Reaktion auf die Revolution in WORRALL, Coping; zu den Auswirkungen auf das Verhältnis zu London vgl. BLACKWELL, Struggle for Jordan, S. 174-9; dennoch gab es auf Seiten der JCS offenbar fortlaufende, wenn auch nicht umgesetzte Planungen über eine mögliche Errichtung von US-Stützpunkten im Raum „East of Suez“, unter anderem auch in Iran ungeachtet des sowjetisch-iranischen Vertrags von 1921. Siehe Nussbaum Memo, 10.10.58, , Iran Defense Affairs - General 1958 3-A', Lot 60 D 533, Subject Files Relating to Iran, 1951-1958, OiCIA/GTI, Box 10.

${ }^{238}$ Vgl. YAQUB, Containing Arab Nationalism, S. $257 \mathrm{f}$. 
peripheren Strategie, dem Versuch, dem Druck der arabischen „Frontstaaten“ Allianzen mit den geographisch weiter entfernten Mächten der Region - Äthiopien, Iran, der Türkei und dem Sudan - entgegenzusetzen. Unter anderem entstand aus diesen Bemühungen noch im Jahr 1958 die geheime TRIDENT-Allianz der iranischen, israelischen und türkischen Geheimdienste, offenbar finanziell unterstützt seitens der CIA. ${ }^{239}$ In Anerkennung der vorteilhaften Entwicklung der Beziehungen erklärte Mohammad Rezā nun auch öffentlich die bereits seit 1950 bestehende de facto-Anerkennung Israels, scharfe Kritik arabischer Nationalisten bewusst in Kauf nehmend. ${ }^{240}$ SAWAK-Direktor Baxtijār bestätigte 1960 die Option, Israel als zusätzliches „Arsenal“ für Irans zukünftige Aufrüstung zu nutzen. ${ }^{241}$

Die Außenpolitik der Eisenhower-Administration in ihren letzten beiden Jahren schloss mit einer weitgehend uninspirierten Bemühung um Schadensbegrenzung, im Grunde das Scheitern sämtlicher eigener Initiativen der Vorjahre implizit eingestehend, die Vermittlungsbemühungen im arabisch-israelischen Konflikt ebenso betreffend wie den angestrebten Aufbau einer kollektiven Verteidigungsorganisation in der Region, die Zurückdrängung des radikalen arabischen Nasserismus oder die Durchsetzung einer unbestrittenen US-Hegemonie im Zuge der Eisenhower-Doktrin. Ähnlich wie in Südostasien oder Lateinamerika vererbte Eisenhower seinem Nachfolger belastete und folgenschwere Beziehungen zu einigen der wichtigsten neuen Akteure in der nun weitgehend dekolonisierten Welt sowie einen angefachten Kalten Krieg entlang der Peripherie. Gerade der Einsatz verdeckter Operationen der CIA mit dem Ziel der interventionistischen Manipulation und Einflussnahme auf die internen politischen Prozesse innerhalb der neuen Staaten und die allzu enge Kooperation mit Sicherheitsorganen amerikanischer Verbündeter sollten prägend auf die zukünftige US-Politik in der sogenannten Dritten Welt wirken. Beides spielte mittelfristig eine katalysierende Rolle in der Ausdehnung der permanenten Interventionspolitik, die letztlich sowohl zum verhängnisvollen militärischen Engagement in Vietnam wie auch zum Aufkommen tiefgreifender zwischengesellschaftlicher

\footnotetext{
${ }^{239}$ Israel versuchte diese Politik gegenüber den USA, teilweise erfolgreich, als unterstützend für den Westen im Kontext des Kalten Krieges zu verkaufen, insbesondere die Bemühungen der Israelis bez. des Ausbaus von „effective internal security services“ in diesen Staaten, um sowohl nasseristische wie kommunistische Umsturzversuche zu bekämpfen. Vgl. MemCon, 21.7.58, FRUS 58-60, XIII, S. 67-72; Ben-Gurion Letter, 24.7.58, ,White House - Meetings [...] 1958 (9)`, JFDP, White House Memoranda Ser., Box 7, DDEL; CIA, Central Intelligence Bulletin, 5.8.58, CREST: \#RDP79*001-6; zu TRIDENT vgl. CIA, ,Israel: Foreign Intelligence and Security Services‘, März 1979, Asnād 11, S. 24; zur Entstehung der Zusammenarbeit und der Rolle der CIA vgl. jetzt ALPHER, Periphery, S. 11-24; bereits im Frühling 1957 trafen die ersten beiden Öllieferungen Irans an Israel im Hafen von Eilat ein, was nach Bekanntwerden zu Protesten der arabischen Staaten führte. Für 1960 schätzte man, dass Iran etwa 86\% der israelischen Erdölimporte bediente. Vgl. Annex A to OCB Progress Report, 21.8.57, ,NSC 5703/1', Policy Papers, Box 43, RG 273; Meyer Memo, 11.2.60, ,1960 Chron.-Inter-Office Memos (2 of 2)', Records of the Director (NE): 1958-1963, Box 1, RG 59, NACP; Ben Gurion pries die mäßigende Wirkung der Geheimdienstkontakte auf den Schah im Anschluss an ein TRIDENT-Treffen in Teheran. Zudem erwähnte er die beginnende Entwicklungszusammenarbeit. Vgl. MemCon, 10.3.60, DDRS: CK2349689101; der TRIDENTBezug fehlt in der Version in FRUS 58-60, XIII, S. 280-8.

${ }^{240}$ Siehe FRUS 58-60, XII, S. $647 n 1$; zur frühen de facto-Anerkennung Israels durch Iran vgl. BIALER, Iranian Connection; offenbar spielte Bestechung iranischer Offizieller eine nicht unerhebliche Rolle bei der Anerkennung Israels. Vgl. SHAwCross, Shah's Last Ride, S. 80f.; vgl. David MENASHRI/Trita PARSI, ,Israel i. Relations with Iran,“ Encyclopcedia Iranica, online edition, New York, 1996-, verfügbar auf http://www.iranicaonline.org/articles/israel-i-relations-with-iran.

${ }^{241}$ CIA Intelligence Support Material, 4.1.60, ,[Iranian Political Situation...][1957-60]‘, WHO, OSANSA, NSC Ser., Briefing Notes Subs., Box 11, DDEL.
} 
Antagonismen wie zwischen den USA und Iran erheblich beitrug. Die Resultate der Nahostpolitik der Eisenhower-Administration taugen in der Tat kaum als Fallbeispiel für eine gemäßigt-konservative und insgesamt erfolgreiche Gesamtstrategie im Sinne des fest etablierten historiographischen Eisenhower-Revisionismus der vergangenen Jahrzehnte. ${ }^{242}$

Belastend war das partielle ,disengagement" Washingtons von der intra-regionalen Politik aber insbesondere für die Mitgliedsstaaten des Bagdadpaktes und gerade für Iran. Im Anschluss an die Suezkrise war der Pakt aufgrund der britischen Mitgliedschaft als Werkzeug des Imperialismus nachhaltig diskreditiert - gerade der Irak als das einzige arabische Mitglied litt unter der propagandistisch offenen Flanke erheblich und John Foster Dulles identifizierte ex post facto die Mitwirkung Bagdads als hauptverantwortlich für die Revolution von $1958 .{ }^{243} \mathrm{Im}$ Kontext der Eisenhower-Doktrin hatte Washington einige Schritte hin zur Aufwertung der eigenen Rolle im Pakt unternommen, z.B. den Beitritt zum Military Committee im Juni 1957 und die direkte Mitwirkung amerikanischer Offizieller an den militärischen Planungen der Allianz. Dabei konzentrierte sich Washington aber weiterhin auf Reaktionen im Falle eines umfassenden sowjetischen Angriffs auf die Gesamtregion und vermied, zuvorderst aus politischen Gründen, gemeinsame Planungen $\mathrm{zu}$ regionalen Bedrohungen. Die Militärplanungen im Falle eines allgemeinen Krieges gegen die Sowjetunion setzten nun noch stärker als zuvor auf den Einsatz von Nuklearwaffen. ${ }^{244}$ Dies trug letztlich der Tatsache Rechnung, dass sowohl Sowjets wie Amerikaner im Kriegsfall problemlos in der Lage sein würden, dem Gegner den Zugriff auf die Ölquellen zu verweigern, was groß angelegte Operationen in der Region letztlich sinnlos erscheinen ließ. Ähnlich konstatierten die britischen Planer, dass , ,[...] should there be a global war, the Middle East would be a subsidiary theatre and events there would not significantly affect its result.“245 Die unmittelbare politische Folge der in erster Linie nuklearen Verteidigungsstrategie für die Nahostregion war eine Absage an die Idee, konventionelle amerikanische Streitkräfte in der Region zu stationieren, um der

${ }^{242}$ Eine Grundsatz-Kritik des Eisenhower-Revisionismus mit Blick auf die Dritte Welt bei Robert J. MCMAHON, Eisenhower and Third World Nationalism; eine kritische Bestandsaufnahme der jüngeren Forschung in ANDERSON, Devil is in the Details; eine insgesamt sehr kritische Bewertung der Nahostpolitik bei STIVERS, Eisenhower and the Middle East; ähnlich SchUlZINGER, Impact of Suez, S. 263f.; kritisch, wenngleich ausgewogen, die Bewertung bei YAQUB, Containing Arab Nationalism, S. 269-76; durchsichtig und wenig überzeugend der Versuch einer Apologetik von Eisenhowers (Südasien-) und Nahostpolitik mittels einseitiger Fokussierung auf die letzten drei Jahre seitens BARRETT, Greater Middle East.

${ }^{243}$ Vgl. Cabinet Meeting Minutes, 18.7.58, FRUS 58-60, XII, S. 80; 373rd NSC Meeting, 24.7.58, ebd., S. 106; zum Effekt der Suezkrise auf die Paktorganisation vgl. YeşıLBURSA, Baghdad Pact, S. 164ff.; CoHEN, Defending the Northern Tier, S. 155-89; das irakische Regime wurde infolge der eigenen Allianz mit dem Suez-Aggressor Großbritannien fast gestürzt. Vgl. YAQUB, Containing Arab Nationalism, S. 57f., 62.

${ }^{244}$ Vgl. FAIRCHILD/POOLE, History of the JCS: VII, S. 136-43; die USA planten militärische Reaktionen auf intraregionale Aggressionen, gemeint waren zuvorderst Ägypten und Syrien, in Kooperation mit den Briten bzw. unilateral. Im September 1957 lagen die Kriegspläne OPLAN 219-57 und OPLAN 215-58 zu allgemeinen bzw. limitierten Szenarien vor. Nach kontroversen internen Debatten wurden beide Pläne im Februar bzw. März 1958 seitens der JCS akzeptiert. Vgl. Memo for Joint State-Defense Conference, 23.7.57, ,Iran (8) ${ }^{\circ}$, WHO, NSCS, Disaster File Ser., Box 66; Cutler Memo, 17.3.58, ,March 1958 (2)‘, WHO, OSANSA,, Special Assistant Ser., Chronological Subs., Box 5; Irwin II Memo, 28.2.58, ,Department of Defense (2)‘, ebd., Subject Subs., Box 3, DDEL.

${ }^{245}$ COS Memorandum, 3.7.56, BDEE A, 3, I, S. 517; auch die Eisenhower-Administration setzte weiterhin auf den Plan einer systematischen Zerstörung der Ölquellen im Falle einer drohenden sowjetischen Übernahme der Golfregion während eines allgemeinen Krieges. Vgl. oben S. 83, Anm. 173; FAIRCHILD/PoOLE, History of the JCS: VII, S. $141 \mathrm{f}$. 
Eisenhower-Doktrin auch militärische Glaubwürdigkeit zu verleihen. Zugleich entfielen angesichts der einseitigen Betonung taktischer Nuklearschläge die militärstrategischen Begründungen für einen Aufbau der Streitkräfte der regionalen Staaten, und die politische Rechtfertigung für Militärhilfe trat noch prägnanter in den Vordergrund. ${ }^{246}$

Die oben erwähnte NSC-Studie 5703/1 vom Februar 1957 mit Bezug auf Iran mit ihrer Betonung einer Steigerung der amerikanischen Hilfsmaßnahmen an Iran war noch stark geprägt gewesen von der ambitionierten Atmosphäre im Zusammenhang mit der Formulierung und anschließenden Propagierung der Eisenhower-Doktrin und der Idee einer hegemonialen Transformation der amerikanischen Rolle in der Region. ${ }^{247}$ Bereits die Ergebnisse der Richards-Mission verdeutlichten, wie wenig realistisch diese Zielsetzungen gewesen waren. Für Iran sprangen zusätzliche Militärhilfe von etwa \$13 Millionen, überwiegend für ,military construction“-Zwecke und \$6 Millionen für Telekommunikationsprojekte und den Ausbau der Eisenbahnverbindungen heraus. ${ }^{248}$ Zusätzlich sagte man den Bau eines modernen Militärflughafens nahe Ġom für weitere \$6.5 Millionen zu. Das anvisierte Military Assistance Program (MAP) für das Etatjahr 1958 über $\$ 40$ Millionen, davon wieder etwa die Hälfte für den Bau militärischer Anlagen und damit nicht für Waffen bestimmt, lag weit unter den iranischen Erwartungen. Die Iraner hatten dem DOD eine Wunschliste mit Ausrüstungsforderungen in einer Höhe von bis zu einer halben Milliarde US-Dollar zukommen lassen und der Schah selbst träumte von einer gewaltigen Ausweitung der Truppenstärke, einer Luftwaffe mit 2.000 Kampfjets, einer schlagkräftigen Marine und von Waffensystemen mit atomarer Kapazität. ${ }^{249}$ Auf Seiten der Amerikaner mehrten sich die Beschwerden über die grandiosen Aufrüstungsphantasien Mohammad Rezās. Sein Eingreifen in Entscheidungen über Fragen der geeigneten Ausrüstung - in der Regel zugunsten hochmoderner Systeme aus

\footnotetext{
246 Tatsächlich erwähnte die neue US-Nahoststrategie NSC 5801/1 vom Januar 1958 das traditionelle Verteidigungskonzept entlang des Northern Tier überhaupt nicht mehr und betonte Wirtschafts- statt Militärhilfe. Vgl. ebd., S. 144; die JCS selbst betonten Ende 1957, dass allein politische und nicht militärische Maßnahmen die Region vor einer kommunistischen Übernahme bewahren könnten und plädierten für ,decisive U.S. political and diplomatic action to solve the present Arab-Israeli dispute. [...] The threat to U.S. security inherent in failing to take the initiative in solving this problem is so great as to transcend the interests of any minority group within the United States.“; JCS Memo, 4.12.57, FRUS 55-57, XVII, S. 838.

${ }^{247}$ NSC 5703/1 hatte explizit die überzogene These aufgestellt, dass , ,[...] the more Iran develops into a positive political and economic asset the greater would be U.S. influence beyond Iran's borders."; NSC 5703/1, 8.2.57, FRUS 55-57, XII, S. 901.

${ }^{248}$ Iran hatte um Projektgelder im Kontext der Doktrin in Höhe von \$102 Millionen angefragt. Vgl. Tehran \#1489, 25.3.57; Karachi \#2632, 28.3.57; Rountree Memo, 4.4.57, FRUS 55-57, XII, S. 922ff., 928-31, 488f.; in GTI konstatierte man nüchtern, ,[...] that we are not prepared to think in program terms really different from those of the past several months. “; Skiles Memo, 24.1.57, ,Iran Middle East Affairs - General 1957', Lot 60 D 533, Subject Files Relating to Iran, 1951-1958, OiCIA/GTI, Box 9; hier begann die US-Unterstützung für eine Eisenbahnverbindung zwischen der Türkei und Iran. Eine durchgehende Strecke wurde schließlich im September 1971 fertiggestellt, für Gesamtkosten von etwa \$100 Millionen über die Jahre. Vgl. Bell Memo, 30.9.59, W/MSCNEA File - 12/58-1959', Lot 61 D 288, Office Files of John O. Bell, 1957-61, Box 1; McDonald Jr. Memo, 30.8.71, ,GTI Miscellaneous 1971', Lot 74 D 281, NEA/Deputy Assistant Secretary, Rodger P. Davies Subject Files, Box 4.

${ }^{249}$ Das MAP-Programm sah bislang 60 Kampfjets für Iran vor. Im Anschluss an den NATO-Beschluss zur Stationierung von Mittelstreckenraketen im Allianzgebiet forderte der Schah ebenfalls die Ausstattung mit MIM3 Nike Ajax-Flugabwehr- und MGR-1 Honest John-Kurzstreckenraketen. Zudem bestand er auf der Lieferung F86 Sabres anstelle der älteren F-84G Thunderjets als Ersatz für die obsoleten 18 P-47 (F-47) ThunderboltPropellermaschinen. Vgl. Seitz Memo, 3.1.57; Rountree Memo, 12.10.57; Tehran \#1164, 15.12.57, FRUS 55-57, XII, S. 866ff., 949ff., 959f., 960 n7; vgl. ebd., S. 877f. n7; Hāğ 'Ali Kijā Letter, 11.9.57, ,Iran U.S. Military Assistance to Iran 1957‘, Lot 60 D 533, Subject Files Relating to Iran, 1951-1958, OiCIA/GTI, Box 10.
} 
Prestigeerwägungen anstelle von militärischer Praktikabilität - erschwerte die Arbeit von ARMISH-MAAG vor Ort. ${ }^{250}$ Botschafter Chapin gestand Ende 1957 seine Frustration über die wiederholten Konflikte mit dem iranischen Monarchen in Militärfragen ein: „We can never really hope to convince $\{$ the; R.P. $\}$ Shah that any given level \{of $\}$ military forces will be adequate to what he imagines are his requirements. “251

Die Anpassungen der breiteren Strategie infolge von Suez und der irakischen Revolution erschwerten Versuche, den Schah zu mehr Mäßigung anzuhalten. Mit NSC 5703/1 hatte die Eisenhower-Administration das Ziel einer Verteidigung der Elburs-Linie, wie lange seitens der Paktstaaten gefordert, auch offiziell angenommen. Allerdings hatte man zugleich Organisation und Umfang der iranischen Streitkräfte für diese Zwecke definiert. Nachdem aber eine Studie aus den militärischen Planungsstäben des Bagdadpaktes, nunmehr mit Beteiligung amerikanischer Offizieller, Forderungen nach einer Aufstockung der iranischen Armee auf 16 vollwertige Divisionen erwähnt hatte, nutzte Mohammad Rezā dies im Anschluss für eine Ausweitung seiner ohnehin bereits exorbitanten Aufrüstungswünsche. ${ }^{252}$ Ein weiterer inoffizieller Besuch in den USA, sein dritter Besuch nach 1949 und 1955, sollte seinen Forderungen im Juli 1958 Nachdruck verleihen. Die Eisenhower-Administration informierte den Monarchen über die ohnehin bereits anvisierten Waffenlieferungen, unter anderem 272 zusätzliche M47 Patton-Panzer und 52 F-86F Sabre-Jagdflugzeuge, ging aber vorerst nicht auf weitere Forderungen ein. ${ }^{253}$ Mit der Revolution im Irak und der Erschütterung des Bagdadpaktes im Juli 1958 veränderte sich die Wahrnehmung in Washington aber schlagartig. In einer ersten Reaktion verständigten sich Dulles und Eisenhower darüber, dem Schah bezüglich der Aufstockung der Streitkräfte entgegenzukommen und die bislang reduzierten Divisionen in vollwertige umzuwandeln. Im Rahmen des Plan Counterbalance würde somit die Armee auf 12 Divisionen verstärkt und die Sollstärke um 37.000 auf 180.000 Mann erhöht werden: „They should have all the assistance they can absorb.“254

${ }^{250} \mathrm{Vgl}$. Admiral Radfords Bemerkungen in 276th NSC Meeting, 9.2.56, FRUS 55-57, XII, S. 803.

251 „His personal psychological bias renders him immune to logical persuasion in this field.“; Tehran \#1195, 18.12.57, ebd., S. 963; Dulles informierte die Botschaft Ende 1957, dass für die Zukunft direkte Budgetzuschüsse so gut wie ausgeschlossen wären. Vgl. State \#1160, 10.12.57, ebd., S. 956ff.

${ }^{252}$ Vgl. JCS History Iran, S. 57ff.; NSC 5703/1 hatte den wünschenswerten Umfang der Streitkräfte auf sechs vollwertige und sechs reduzierte Divisionen sowie fünf unabhängige Brigaden festgelegt. Vgl. oben S. 171, Anm. 194; die JCS sahen „,no military requirement“ für einen Umfang jenseits von zehn Divisionen, ungefähr äquivalent zu dieser Größenordnung. Das State Department dagegen empfahl eine Zusage für die Ausstattung von elf ,vollen“ Divisionen bis 1965 mit jährlichen Zusatzkosten von \$50 Millionen. Das DOD sprach sich für die Lieferung atomwaffenfähiger Honest Johns anstelle einer zusätzlichen konventionellen Abschreckung aus, wobei die nuklearen Sprengköpfe außerhalb Irans gelagert werden sollten. Vgl. State Talking Paper, 11.7.58, ,Iran Military Assistance - General 1958 3-A/1', Lot 60 D 533, Subject Files Relating to Iran, 1951-1958, OiCIA/GTI, Box 10. ${ }^{253}$ Vgl. JCS Memo, 9.6.58; Background Info, encl. to Dulles Memo, 28.6.58, FRUS 58-60, XII, S. 554-60, 562-5; die Sabres sollten bis 1961 die iranischen F-84Gs ersetzen, womit eine seit Längerem bestehende Forderung des Schahs erfüllt worden wäre. Zusätzlich sagten die USA 14 neue Aufklärungsflugzeuge zu. Die USA hatten bereits im Januar 1958 im Anschluss an einen Besuch von Dulles in Teheran zusätzliche \$14 Millionen Militärhilfe gewährt. Vgl. MemCon, 22.1.58; Tehran \#Dulte7, 25.1.58, ebd., S. 531f., 533ff.; Irwin Memo, 25.1.58; MemCon, 25.1.58, ,Iran Military Assistance [...] 3-A/1', Lot 60 D 533, Subject Files Relating to Iran, 1951-1958, OiCIA/GTI, Box 10; J.C.S. 1887/460, 11.6.58, ,CCS 381 E.M.M.E.A. (11-19-47) Sec 70', JCS Geographic File 1958, Box 3, RG 218, NACP.

${ }^{254}$ TelCon, 16.7.58, ,Telephone Calls - July 1958‘, AWF, DDE Diary Ser., Box 34, DDEL; ARMISH-MAAG Tehran \#ARGC76253, 19.8.58; Twining Memo, 9.7.58, ,CCS 092 Iran CENTO (4-23-48), Sec. 21“, JCS Geographic File 1958, Box 8, RG 218, NACP; vgl. State \#204 [Eisenhower Letter to Shah], 19.7.58; Barnes 
Während Mohammad Rezā das amerikanische Entgegenkommen wenig überraschend als endgültige Zustimmung Washingtons zu seinen Aufrüstungswünschen interpretierte, zumal er nun zusätzlich eine direkte regionale Bedrohung Irans durch einen nasseristischen Satelliten an der eigenen Westgrenze anführen konnte, wandelte sich die Stimmung innerhalb der Eisenhower-Administration rasch. Eine Bestandsaufnahme des „Verlusts“ Bagdads musste zwangsläufig die Aufmerksamkeit der Planer auf die von amerikanischer Seite nur unzureichend verstandenen wie beachteten innenpolitischen Prozesse in den verbündeten Staaten im Nahen und Mittleren Osten lenken. Bereits Anfang 1956 hatte William Rountree von NEA im State Department konstatiert, dass eine Steigerung der Militärhilfe immer den Beifall des Schahs und der Armeeführung erhalte, aber das diese Art der Unterstützung kaum einen Effekt auf die iranische Öffentlichkeit habe. ${ }^{255}$ Tatsächlich hatte der Impuls zugunsten einer reformistischen Intervention in Iran trotz der weitgehenden Bekräftigung der bisherigen Iranpolitik in der NSC-Studie 5703/1 vom Februar 1957 auf der mittleren und unteren Ebene der US-Administration weiter an Schwung gewonnen und die internen Debatten über die Umsetzung der Studie bzw. einen Anpassung des „Outline Plan of Operations“ für Iran plädierten ein weiteres Mal für die Gewinnung der „dissatisfied Nationalist groups“ mittels eines ,gradual improvement in government, involving the adoption of internal reforms and the building of government institutions which enjoy greater popular confidence“. ${ }^{256}$ Kurzzeitig hofften die Amerikaner auf die im April 1957 berufene neue Regierung unter dem als sehr proamerikanisch geltenden Manučehr Eg̉bāl und der OCB empfahl eine Reihe von Schritten, um den Schah zu ermuntern, die zugesagte Handlungsautonomie des neuen Kabinetts auch wirklich zu akzeptieren. ${ }^{257}$ Bald zeigte sich aber, dass auch Eg̈bāl keinerlei Spielraum erhielt und sich stattdessen öffentlich als serviler Untertan des Monarchen gerierte. Der ohnehin bestehende Scheinkonstitutionalismus in Iran wurde nun gekrönt durch die Einführung eines begleitenden Scheinpluralismus, einzig und allein infolge eines Befehls Mohammad Rezās. Der Schahvertraute Asadollah 'Alam schuf die Mardom-(Volks-)Partei, gefolgt von der Partei der Nationalisten (Hezb-e Mellijun) Eg̉bāls. Dem britischen Zweiparteiensystem nachempfunden,

Memo, 24.7.58, FRUS 58-60, XII, S. 573f., 579ff.; vgl. ebd., S. 581 n3; zugleich wurden die vorgemerkten Waffensystemlieferungen beschleunigt, was zusätzliche Kosten von bis zu \$50 Millionen generierte. Der Schah zeigte sich sehr zufrieden mit der neuen Entwicklung, bedauerte aber die Lieferung der F-86s, da er inzwischen die noch moderneren F-100 Super Sabres favorisierte. Eisenhower hatte wenige Tage nach dem Coup die Idee einer 12-14 Divisionen-Armee für Iran unterstützt. Dulles sprach sich gar für 16 Divisionen aus. Vgl. MemCon, 17.7.58; MemCon, 16.7.58, ebd., S. 76f., 72-5; am Tag der Revolution bekräftigte Dulles die Zentralität der USBeziehungen zur Türkei, zu Iran und zu Pakistan: „We always felt we would lose the Arab world but did not want to under circumstances that would lose the confidence of these countries."; TelCon, 14.7.58, FRUS 58-60, XI, S. 209.

${ }^{255}$ Vgl. MemCon, 13.1.56, FRUS 55-57, XII, S. 227, hier; Rountree war zu dieser Zeit Deputy Assistant Secretary of State for Near Eastern, South Asian, and African Affairs, ab Juli 1956 dann als Nachfolger George Allens Assistant Secretary.

${ }_{256}$ OCB Study on Operational Guidance, 22.5.57, ,OCB 091. Iran (File \#5) (3)‘, WHO, NSCS, OCB Central File Ser., Box 44, DDEL.

${ }^{257}$ Man solle den Schah überzeugen, aber nicht zwingen, „, to give real backing to the Prime Minister when the latter embarks on courses of action which are temporarily unpopular with the traditional ruling groups [...]" und den Schah ermuntern, seine „supervision of daily affairs“ aufzugeben. Ebd;; der reformistische Impuls noch abgeschwächter in OCB Operations Plan, 30.4.58, , Iran - 1958', Lot 62 D 430, Regional and Country Operations Files, 1953-1961, Box 20; zu Eg̉bāl vgl. Milani, Eminent Persians: I, S. 124-8. 
war für die Iraner selbst der gespielte Gegensatz zwischen Mardom und Mellijun eine allzu augenfällige Farce - die Bevölkerung nannte die beiden Parteien spöttisch die „Ja-Partei“ und die ,Ja, mein Herr-Partei“ - und der Schritt diskreditierte den Herrschaftsstil Mohammad Rezā nachdrücklich. Der NSC-Stab warnte im Herbst 1957: „His disinclination to assume the role of a constitutional monarch and the consequent criticism directed at him, rather than against his government and ministers, may serve to weaken his position and that of the monarchy over a period of time. “258

Die kritischen Stimmen zum Schah innerhalb der Eisenhower-Administration wurden lauter. Ein von der US-Botschaft in Teheran erstelltes umfassendes Psychogramm Mohammad Rezās geriet wenig schmeichelhaft. So sei seine frühere Unsicherheit großem Selbstbewusstsein gewichen, doch seien viele seiner bereits altbekannten charakterlichen Schwächen wie mangelnde Entschlusskraft und Empfänglichkeit für Sykophanten weiterhin prägnant. Zudem konstatierte es eine Tendenz zur aktivistischen und absolutistischen Herrschaft und eine fast schon paranoide Angst vor einem populären politischen Rivalen, was ihn zwanghaft zur Schwächung und Unterminierung jeder Regierung bewege, und ihn damit zum Haupthindernis für wirtschaftlichen Fortschritt und Demokratisierung mache. ${ }^{259}$ Der scheidende USBotschafter Selden Chapin berichtete nach Washington über die Zufriedenheit des Schahs, endlich eine ,position of commanding power“ erlangt zu haben - das Country Team in Teheran war noch expliziter: „No further question that $\{$ the; R.P. $\}$ Shah is $\{$ the $\}$ de facto dictator of Iran.“260 Ein OCB-Bericht vom April 1958 konstatierte, die interne Stellung des Schahs sei geschwächt und die Zusammenarbeit mit ihm gestalte sich zunehmend schwieriger. ${ }^{261}$ Die CIA bestätigte nun die ,basic and widespread dissatisfaction with his regime, both in the army and in the urban population generally." 262 Im August warnte DCI Allen Dulles den NSC, dass eine

\footnotetext{
${ }^{258}$ Rountree Memo, 7.9.57, FRUS 55-57, XII, S. 940f.; die Bezugnahme ist auf den Progress Report des OCB zu NSC 5703/1 vom August 1957, der konstatierte, ,[...] the Shah's vacillation and indecision continue to inhibit attempts to improve the quality of government."; OCB Progress Report on Iran, 21.8.57, ,NSC 5703/1", Policy Papers, Box 43, RG 273, NACP; zu den beiden neuen Scheinparteien vgl. ANSARI, Modern Iran, S.139f.; AZIMI, Crisis, S. 161-4; ders., Parties in Iran, S. 65f.; Gasionowski, Client State, S. 160f.; zu 'Alam vgl. Milani, Eminent Persians: I, S. 44-55.

${ }^{259}$ Die Studie bestätigte in diesem Punkt eine Vorgängerstudie aus dem Jahr 1951. Vgl. Study ,The Shah of Iran, 1957‘, encl. to Tehran \#806, 11.3.57, FRUS 55-57, XII, S. 910-20; die Studie erwähnte die Entfernung seines Schweizer Jugendfreundes und politischen Intriganten Ernest Perron vom Hof als wichtigen Schritt seiner charakterlichen Entwicklung. Zu Perron vgl. MeIER, Swiss Gardener; der Scheinpluralismus habe die iranischen Reformisten zutiefst enttäuscht, zumal politische Aktivität nunmehr nur noch im Rahmen dieser beiden Parteien erlaubt sei, ,enforced by the SAVAK with police methods.“; OCB Progress Report, 8.10.58, ,NSC 5703/1 ‘, Policy Papers, Box 43, RG 273, NACP.

${ }^{260}$ Der Schah, so Chapin, ,[...] has increasingly taken over decision making on all important and many minor questions."; Tehran \#2200, 29.5.58, FRUS 58-60, XII, S. 549; Tehran \#496, 4.12.57, 611.88/12-457, CF 55-59; Eg̉bāl sei , ,[...] discredited and reduced to role of puppet."; der Schah halte Eg̈bāl an einer sehr kurzen Leine. Eg̉bāl selbst sei „,learly frustrated and uncomfortable“ und würde inzwischen mit entwaffnender Offenheit die politisch Elite und auch den Schah selbst kritisieren, berichtete Stevens. Stevens Letter, 31.5.57, FO 371/127075, TNA:PRO.

${ }^{261}$ Vgl. Harr Memo, 8.5.58, FRUS 58-60, XII, S. 547f.; der OCB hatte berichtet, es gäbe ,little or no progress in achieving a more stable government in Iran.“; Mohammad Rezā zeige nun eine „marked reluctance to accept American advice" und bezichtige amerikanische Offizielle der geheimen Kooperation mit Oppositionellen. OCB Progress Report, 2.4.58, ,NSC 5703/1 ', Policy Papers, Box 43, RG 273, NACP.

${ }_{262}$ CIA, SNIE 34-58, 26.8.58, ,379th Meeting', Official Meeting Minutes, Box 22, RG 273, NACP; CIA NSC Briefing, 9.9.58, CREST: \#RDP79*003-8; die CIA sagte als „,most probable development“ einen Staatsstreich durch Offiziere, womöglich im Verbund mit liberalen Oppositionselementen, voraus. Auf britischer Seite verwahrte sich die Botschaft gegen eine Geheimdiensteinschätzung, die iranische Armee würde im Falle eines
} 
Entwicklung in Iran analog zum Militärputsch im Irak nicht mehr auszuschließen sei: ,„[...] we should face the possibility that unless the Shah puts into effect some dramatic internal reforms, his days will be numbered.“ Man müsse den Schah zu weitreichenden Reformschritten ermuntern, solange noch Zeit dafür sei, so seine Empfehlung. ${ }^{263}$ Die Grundidee des reformistischen Interventionismus hatte nun die allerhöchste Führungsebene erreicht.

In der nun folgenden bürokratischen Auseinandersetzung meldeten sich aber nun die traditionellen Unterstützer der engen Anbindung der amerikanischen Iranpolitik an Person und Herrschaft Mohammad Rezās zu Wort und das State Department bekräftigte erneut die Sichtweise ,[...] that the Shah of Iran represents the best hope for evolutionary and peaceful change." Befürworter einer Sicherung der pro-westlichen Monarchie nach innen argumentierten, nach gewohntem Schema, nun wieder zugunsten einer Aufstockung der amerikanischen Hilfsleistungen, insbesondere einer Rücknahme der Absichtserklärung, Finanzhilfen an Iran in Gänze einzustellen. Im NSC Planning Board wurden gleichzeitig Überlegungen angestrengt, wie man ein „rapprochement“ zwischen dem Schah und der Opposition herbeiführen könne bzw. welche Mittel den USA zur Verfügung standen ,,to alter the present system of personal government by the Shah". ${ }^{264}$ Insbesondere das State Department und die Botschaft in Teheran wehrten sich nachdrücklich gegen eine Aufgabe der Schahzentrierten bisherigen Politik und behaupteten, weitgehend gegen besseres Wissen, positive Entwicklungen in der jüngsten Vergangenheit, die Anlass gäben zu, ,[...] cautious optimism that regime may with luck and skill consolidate its position and is not inevitably doomed.“265 OCB und CIA aber blieben skeptisch bezüglich des tatsächlichen Reformwillens Mohammad

Coups das Regime nicht verteidigen. Das Foreign Office war nicht überzeugt: „As in Iraq, all that is needed is a few bold spirits in charge of a few strategically-located units for a few hours and the rest of the Army will be faced with, and almost certainly accept, a fait accompli."; D.T. West Minute, 12.9.58; FO to Tehran, No. 1152, 8.8.58; Stevens to FO, No. 657, 11.8.58, FO 371/133006, TNA:PRO.

${ }^{263}$ Zit. aus ed. note, FRUS 58-60, XII, S. 585; die Botschaft berichtete über die tiefe Verunsicherung des Schahs nach dem Sturz der Haschemiten in Bagdad und die neue Zuversicht der verschiedenen Oppositionsgruppen: „Mosadeq-type individuals and small opposition groups in Tehran are markedly more confident and ubiquitous than before the coup."; Tehran \#395, 14.8.58, ebd., S. 583; die Botschaft begab sich auf die Suche nach dem iranischen "Qāsim“. Zugleich zeigte man sich vor Ort skeptisch, was die Möglichkeit eines „Konstitutionellen Coups" anging, also eines ,soften“ Staatsstreichs mit dem Ziel, den Schah in seine verfassungsmäßige Rolle zurückzudrängen. Tehran \#176, 4.9.58, 788.00/9-458; Tehran \#279, 21.10.58, 788.00/10-2158, CF 55-59; USMilitärs vor Ort gaben sich überzeugt, dass die Armee im Falle eines Staatsstreiches den Schah nicht verteidigen würde. Siehe CIA, Central Intelligence Bulletin, 8.8.58, CREST: \#RDP79*001-3; in der Umgebung des Schahs gab es Individuen, denen die dringliche Notwendigkeit umfassender Reformen, um das Regime zu retten, bewusst war. Der Schah-Vertraute 'Alam forderte im Gespräch mit einem britischen Offiziellen einen Austausch des Führungspersonals und eine „Weiße Revolution“ von Oben inklusive Landreform, die dann später 1963 verwirklicht werden sollte. Siehe Stevens Letter, 11.8.58, FO 371/133006, TNA:PRO.

${ }^{264}$ Smith Memo, 5.9.58, FRUS 58-60, XII, S. 587; der Nationale Sicherheitsberater Gordon Gray fasste das Resultat der NSC Sitzung im September lapidar zusammen: „The Shah of Iran was likely to be removed from power but there was nothing the U.S. could do about it.“; 379th NSC Meeting, 18.9.58, ebd., S. 593; Gray Memo, 26.8.58, , Meeting with the President-1958 (3)‘, WHO, OSANSA, Special Assistant Ser., Presidential Subs., Box 3; J.I.C. Memo, 8.9.58, ,Chronological - Karl G. Harr, August-December 1958 (2)‘, WHO, OSANSA, OCB Ser., Administrative Subs., Box 1; Planning Board Draft Briefing Memo, 8.9.58, ,Iran (2)“, WHO, NSCS, Special Staff File Ser., Box 4, DDEL; im NSC kritisierte man zudem das völlige Fehlen von Plänen, wie sich die USA im Falle einer geglückten oder versuchten Machtübernahme indigener nationalistischer Elemente in Iran verhalten sollte. ${ }^{265}$ Tehran \#740, 11.10.58, FRUS 58-60, XII, S. 595; Rountree bezeichnete das vorherige SNIE gegenüber Dulles als zu ,gloomy“. Ed. note, ebd., S. 596f;; siehe auch die optimistischen Bemerkungen des Acting Secretary of State Herter in der NSC-Sitzung. Siehe vorherig. Anm. 
Rezās. ${ }^{266}$ In einer grundsätzlichen Abkehr von der bisherigen Sichtweise definierte die vierte NSC Studie zu Iran der Eisenhower-Administration, NSC 5821/1 vom November 1958, als „key problem“ nicht mehr die Rückversicherung des pro-westlichen Hofs bzw. die militärische Bedrohung seitens der UdSSR, sondern nunmehr ,[...] the extent to which the largely personal regime of the Shah of Iran, with which the United States is now closely identified, can cope successfully with Iran's growing internal problems." Ohne substantielle politische, wirtschaftliche und soziale Reformen, so die Studie, sei die iranische Monarchie „likely to be overthrown“. ${ }^{267}$ Interessant war insbesondere die Betonung der möglichen strategischen Folgen eines nationalistischen Umsturzes aufgrund der ,possible over-identification of the U.S. with the Shah's policies“, die durchaus , ,[...] may reach the point where this can seriously endanger U.S. objectives in Iran“, wie der OCB bereits früher gewarnt hatte. ${ }^{268}$ Wenngleich im NSC Planning Board Einigkeit bestand über die hauptsächliche Bedrohung in Iran, gab es zugleich eine kontroverse Diskussion über die adäquate US-Reaktion - Uneinigkeit bestand, ob man den Schah, wie bisher, mit ,inducements“ zu mehr Reformen mit dem Ziel der politischen Kooptation der ,growing educated middle classes“ bewegen sollte oder ob angesichts der Dringlichkeit nicht die Anwendung von ,pressure“ angeraten sei. NSC 5821/1 enthielt schließlich als zentrale Richtlinie der neuen Politik folgende Kompromissformel:

Recognizing that a stable progressive regime under the pro-Western Shah of Iran would best serve U.S. interests, make a sustained effort to induce, and, where appropriate, press the Shah to institute promptly, meaningful political, social and economic reforms designed to increase popular support for his regime $[\ldots] .^{269}$

Die reformistische Intervention war damit im November 1958 zur offiziellen Leitlinie der amerikanischen Iranpolitik geworden. Auf den zweiten Blick aber erschien der bürokratische Sieg der Reformisten als Pyrrhussieg. Die in der NSC-Studie aufgelisteten Reformschritte gingen nicht allzu weit und fokussierten auf als dringend empfundene vorwiegend soziale und

\footnotetext{
${ }^{266}$ Der Schah hatte Maßnahmen gegen die grassierende Korruption angekündigt und eine Beschränkung der stark kritisierten wirtschaftlichen Aktivitäten der kaiserlichen Familie und der Höflinge angedeutet. Der OCB blieb skeptisch: „Although the Shah has indicated increasing awareness of the situation, of particular concern is the Shah's ingrained tendency towards self-delusion and his everpresent reluctance seriously to antagonize vested interests.“; OCB Progress Report, 8.10.58, ,NSC 5703/1“, Policy Papers, Box 43; vgl. Draft OCB Progress Report on Iran, 8.9.58, ,OCB 091 Iran', Records of the OCB, Box 3, RG 273, NACP; die britische Botschaft sah die zögerlichen Reformschritte vom Herbst 1958 weitaus positiver, insbesondere sein Eingreifen gegen das zweifelhafte Wirtschaftsgebaren der kaiserlichen Familie und insbesondere seiner Zwillingsschwester Ašraf: „The unhappy monarch appears at last to have steeled himself against his racketeering family."; Russell to Lloyd, No. 146, 27.10.58, FO 371/133007, TNA:PRO; die CIA bekräftigte die Schlussfolgerungen des SNIE ein weiteres Mal im November: „Even if the Shah does carry out recent promises to put an end to official corruption and does effect significant economic and social reforms, we do not believe that the general discontent will be stilled or the opposition drop its plans for action against him unless he modifies the autocratic character of his rule and provides more opportunity for participation in government."; dafür gebe es aber keinerlei Anzeichen und die CIA erwartete einen „,move“ gegen ihn „within a year or so“; ONE Memo to DCI, 10.11.58, FRUS 58-60, XII, S. 597-60.

${ }^{267}$ NSC 5821/1, Statement of U.S. Policy Toward Iran', o.D. [angenommen vom NSC am 13.11.58], ebd., S. 60515, hier $605 f$., 607.

${ }^{268}$ OCB Progress Report, 8.10.58, ,NSC 5703/1‘, Policy Papers, Box 43, RG 273, NACP.

${ }^{269}$ NSC 5821/1, S. 612; an anderer Stelle war die Rede war von einer ,delicate balance between pressure and persuasion".; ebd., S. 607; allein das State Department wehrte sich gegen die Erwähnung von jeder Art von „,pressure“. Die Debatten im NSC Planning Board nachgezeichnet in PB Briefing Papers, 23./27./30.10.58, ,Iran (2)‘; PB Briefing Paper, 12.11.58, ,Iran (1)‘, WHO, NSCS, Special Staff File Ser., Box 4, DDEL.
} 
wirtschaftliche Maßnahmen wie Steuer- und Landreform. In politischer Hinsicht war von einer Liberalisierung legislativer und judikativer Praktiken, aber nicht von freien Wahlen, die Rede. Explizit wurde die ,absence of any constructive, pro-Western alternative“ in Iran herausgestellt, verwunderlich angesichts der in ihren liberalen und häufig demokratischen Vorstellungen durchaus westlich inspirierten nationalistischen Opposition. Nur im Falle einer offenkundigen Weigerung des Schahs zur Durchführung von Reformen bzw. sollte ein Sturz seiner Herrschaft absehbar sein, empfahl NSC 5821/1 Schritte, um die Identifikation der USA mit dem Regime zu vermindern und sich einer Nachfolgeregierung anzunähern. ${ }^{270}$ Der Schah war weiterhin die „best hope“ für das Erreichen amerikanischer Politikziele und NSC 5821/1 damit eher eine Fortsetzung der bisherigen Politik mit reformistischen Anpassungen denn eine wirkliche politische Wende. Fatal sollten sich die pfadabhängigen Effekte der eigenen politischen Weichenstellungen der Vorjahre für den reformistischen Impuls auswirken. Die Zusagen über erhöhte militärische Unterstützungsleistungen und die Anhebung der Sollstärke der Streitkräfte untergruben die Wünsche nach stärkerer Konzentration auf wirtschaftliche und soziale Aspekte und trugen maßgeblich zur weiteren Destabilisierung der finanziellen Situation des Landes bei. Im Zuge der steigenden Einnahmen aus der Ölförderung (allein \$260 Millionen im Etatjahr 1959) hatten sich die Staatsausgaben seit 1955 verdoppelt, die Verteidigungsausgaben sogar fast verdreifacht. Für 1959 wurden gewaltige Budgetdefizite vorausgesagt und der Schah hatte bereits mehrfach signalisiert, dass er von seinem amerikanischen Verbündeten erwartete, diese auszugleichen. Ein Konflikt war somit vorprogrammiert. ${ }^{271}$

Angesichts dieser Gegensätze war die in NSC 5821/1 geäußerte Hoffnung, man könne den Schah überzeugen, dass der ,most immediate threat to his regime lies in internal instability rather than external aggression“, und „military forces beyond Iran's ability to absorb and support“ zwangsläufig ,self-defeating“ seien, kaum gerechtfertigt. Trotz der Zusagen im Anschluss an die irakische Revolution verweigerten sich, zum Unwillen Mohammad Rezās, die US-Vertreter im Military Committee den Wünschen des Schahs nach einer zusätzlichen Aufstockung der Sollstärke der iranischen Armee auf nunmehr 20 bis 24 Divisionen, was einer de facto-Verdoppelung gleichgekommen wäre. ${ }^{272}$ Im Dezember 1958 forderte dann

\footnotetext{
${ }^{270}$ NSC 5821/1, S. 607f., 612; erst für den Fall einer anstehenden Entmachtung des Schahs sollten ,,appropriate contacts with any emerging non-Communist opposition groups" etabliert werden. Zugleich war man sich bewusst, dass ein solcher Schritt ,would probably insure the Shah's downfall [...].“; die JCS hatten für die Formulierung plädiert, die USA sollten, ,[...] be prepared to encourage a government friendly to the United States under another pro-U.S. leader, if one is available." \{Hervorheb. i. Orig.\}; JCS Suggestion, o.D., ,[Iranian Political Situation...", WHO, OSANSA, NSC Ser., Briefing Notes Subs., Box 11, DDEL.

${ }^{271}$ Vgl. OCB Progress Report, 8.10.58, ,NSC 5703/1‘, Policy Papers, Box 43, RG 273, NACP; die Ausgaben der Planorganisation waren von \$20 Millionen in 1955 auf \$180 Millionen jährlich angewachsen. Vgl. NSC 5821/1, S. 609ff.; allein im laufenden Jahr sei der Verteidigungsetat im Vergleich zum Vorjahr um $40 \%$ auf etwa $\$ 135$ Millionen angestiegen. Zudem war ein weiterer Anstieg auf \$173 Millionen innerhalb von zwei Jahren geplant. Das State Department hatte zudem die Aufnahme eines Paragraphen (No. 37) in die Studie durchgesetzt, der weitere Budgetzuschüsse implizierte. Vgl. ebd., S. 614; Planning Board Briefing Note, 12.11.58, , Iran (10)', WHO, NSCS, Disaster File Ser., Box 66, DDEL; ein Aufbau der Streitkräfte ohne Gewährung von Budgetzuschüssen ,[...] may very well have a self defeating effect", warnte das State Department. Barnes Memo, 20.10.58, ,Misc (1959-1963)“, Entry A1 3063, Office of the Deputy Under Secretary of State for Political Affairs, MDAP Memos, Box 1, RG 59, NACP.

${ }^{272}$ Vgl. Defense Working Group Member Submission to OCB, 5.9.58, ,Iranian Political Situation...[1957-60]', WHO, OSANSA, NSC Ser., Briefing Notes Subs., Box 11; Draft Memo for Record, 3.2.59, ,Iran (1) [January-
} 
Planorganisation-Direktor Ebtehāğ zusätzliche Militär- und Wirtschaftshilfe von bis zu \$100 Millionen, zuvorderst begründet mit den Belastungen des Plan Counterbalance und der neuartigen Bedrohung seitens der angeblichen sowjetischen Satelliten Irak und Afghanistan. Die Eisenhower-Administration verweigerte daraufhin zusätzliche Unterstützung in einer scharf formulierten Instruktion an die US-Botschaft in Teheran, gleichzeitig warnend, dass die Haushaltskrise „katastrophale Folgen“ haben könnte. Auf der bilateralen Ebene kritisierte der Schah scharf die seines Erachtens unzureichende amerikanische Unterstützung für den Bagdadpakt im Anschluss an die irakische Revolution - die im Sommer 1958, als Ersatz für einen Beitritt, zugesagte Verabschiedung bilateraler Verträge der USA mit Pakistan, der Türkei und Iran verzögerten sich zudem und waren ohnehin aus Sicht Teherans ungenügend, gerade was die Stärke der in den Entwürfen enthaltenen Beistandsverpflichtungen anging. ${ }^{273} \mathrm{Zu}$ Beginn des Jahres 1959 gerieten die iranisch-amerikanischen Beziehungen daraufhin in eine schwere Krise. ${ }^{274}$

Nur wenige Wochen nach Verabschiedung der neuen Strategie bez. Iran zeigte sich mit Nachdruck, wie schwierig es sein würde, Mohammad Rezā zu überzeugen, dass ,his interests and those of the United States are parallel, and that we are seeking to strengthen his regime rather than undermine it." 275 Die Botschaft hatte bereits ein halbes Jahr zuvor vor den Folgen einer Kürzung der Militärhilfe gewarnt - die fast schon pathologische Unsicherheit des Schahs sei nur mittels materieller Unterstützung, ,preferably military hardware“, zu beschwichtigen. ${ }^{276}$ Darüber hinaus war es auch Mohammad Rezā nicht verborgen geblieben, dass sein Ansehen in Washington stark gelitten hatte und innenpolitische Gegner verstärkt auf eine Kehrtwende in der US-Politik hofften. So nahm der seit den Ölverhandlungen in den USA hochgeschätzte 'Ali Amini Kontakt zu Teilen der nationalistischen Opposition auf - „his name is constantly

August 1959]', WHO, SS, International Ser., Box 8, DDEL; einen Vorschlag des DOD, aus taktischen Gründen die vorgeschlagenen ,force levels“ des Bagdadpaktes zu akzeptieren, ohne aber US-Militärhilfe zuzusagen, lehnte das State Department ab. Sicherheitsberater Gray sei „still disturbed“ über die militärischen Bestandteile von NSC 5821/1 und darüber hinaus sei der Planning Board, virtuously unanimous in opposing any further increases in the Iranian armed forces.“; bereits im April 1959 kursierten innerhalb des DOD allerdings Informationen über bestehende iranische Sollstärken von über 200.000 Mann. Vgl. Williams Memo, 10.1.59, ,Office Memoranda1959‘; Perkins Memo, 1.4.59, ,U.S. Military Assistance to Iran 1959‘, Lot 61 D 407, Suppl. Subject Files Relating to Iran, 1956-59, OiCIA/GTI, Box 21.

${ }^{273}$ Vgl. Tehran \#984, 24.11.58, 788.5/11-2458, CF 55-59; Tehran \#G-115 18.12.58, 611.88/12-1858, CF 55-59; vgl. BARRETT, Greater Middle East, S. 152f.; zur iranischen Enttäuschung über die Entwicklung des Pakts vgl. DIMITRAKIS, Failed Alliances, S. 139-48; zu den bilateralen Verhandlungen vgl. RAMAZANI, Iran's Foreign Policy, S. 280-5; die JCS empfahlen Ende 1958 ein weiteres Mal vergeblich einen US-Beitritt zum Pakt. Siehe JCS Memo, 15.1.59, ,CCS 9070 Baghdad Pact, CENTO (15 Jan 1959)`, JCS Central Decimal File 1959, Box 118, RG 218, NACP; vgl. FAIRCHILD/POOLE, History of the JCS: VII, S.170ff.; eine spätere Einschätzung der tatsächlichen Gefahr vonseiten der beiden Nachbarstaaten war eindeutig: ,The idea of a formal armed aggression by the two hypothetical satellites is fantastic."; Handschriftliche Notiz, o.D., ,3-A/1 Military Assistance, Iran 1960‘, NEA, GTI: RIAD, 1958-63, Box 2.

${ }^{274}$ Vgl. Rountree Memo, 3.12.58; State \#1774, 16.1.59, FRUS 58-60, XII, S. 615-9, 622ff.; State \#1772, 16.1.59, 788.5-MSP/1-459, CF 55-59; Tehran \#988, 24.11.58, 788.00/11-2458, CF 55-59; Weiss Memo, 14.1.59, ,Iran, Pakistan, Turkey JMW“, Lot 61 D 288, Office Files of John O. Bell, 1957-61, Box 17.

${ }^{275}$ NSC $5821 / 1$, S. 612 .

${ }_{276}$ Tehran \#1076, 9.6.58, 788.5-MSP/6-958, CF 55-59; in einer Vorwegnahme von NSC 5821/1 warnte die Botschaft, dass „hypertrophied Iranian military forces and plans would be dangerous to the regime and consequently obstructive to U.S. aims."; der gegenwärtige Streitkräfteumfang von 140.000 Mann "has reached a point where it weighs heavily upon the economy of the country and creates potential political dangers."; die geplante Aufstockung auf fast 180.000 Mann bis 1961 sei hochriskant. 
being mentioned mysteriously by people who might someday be dangerous" - und innerhalb des NEA hatte man keinen Zweifel, dass er auf US-Unterstützung für eine Berufung zum Premier spekulierte. ${ }^{277}$ Der Schah selbst beschwerte sich im Laufe des Jahres 1957 beim CIA Station Chief und bei Botschafter Chapin über amerikanische Kontakte zu Personen aus dem NWI-Umfeld. ${ }^{278}$ In diese Atmosphäre des gegenseitigen Misstrauens fiel im Februar 1958, also noch vor der Einleitung des reformistischen Interventionismus, die Ġarani-Affäre. Generalmajor Wali-ollāh Ġarani, Kommandeur des militärischen Geheimdiensts (G-2) in der iranischen Armee, war Ende Februar 1958 verhaftet und illegaler politischer Aktivitäten beschuldigt worden. Iranische Offizielle und Presse deuteten unmittelbar im Anschluss CoupAbsichten Garanis an, in Kooperation mit einer ausländischen Macht, was sich in diesem Fall offensichtlich auf die USA bezog. Nachdem Ġarani im Sommer 1958 zu drei Jahren Haft verurteilt worden war, verschwand die Geschichte rasch aus den Schlagzeilen und die Vorwürfe gegen die USA wurden zurückgenommen, doch führte die Affäre zu einer erheblichen Belastung der amerikanisch-iranischen Beziehungen. ${ }^{279}$

Die Garani-Gruppe strebte offenbar eine Art Palastcoup an mit dem Ziel, den Schah zurück in seine konstitutionelle Rolle $\mathrm{zu}$ zwingen und eine effiziente und autonome Regierung einzusetzen, um grundlegende Reformen in Iran durchzusetzen. Ġarani hatte diese Ziele seit 1956 relativ offen vertreten und sich ein Unterstützungsnetzwerk in der Politik und in der Armee aufgebaut - sowohl amerikanische wie britische Vertreter vor Ort waren mit den Vorgängen gut vertraut und das galt sicherlich auch für den SAWAK. ${ }^{280}$ Mohammad Rezā war

${ }^{277}$ Bowling Letter, 3.9.57, ,Iran Official-Informal [...] 1957‘; Mouser, III, Letter, 18.9.57, ,Correspondence with Post (Official-Informal) 1957‘, Lot 60 D 533, Subject Files Relating to Iran, 1951-1958, OiCIA/GTI, Box 9; vgl. auch FRUS 55-57, XII, S. 779 n3.

${ }^{278}$ Vgl. MemCon, encl. to Stevens Letter, 14.1.57, ebd., S. 868-71; State \#922, 5.11.57, 611.88/11-457, CF 55-57; Tehran \#926, 9.11.57, 611.88/11-957; Chapin bestätigte Kontakte auf unteren Ebenen allein zu dem Zweck, ,to ascertain whether they might be up to anything which might endanger the regime or our mutual relations."; Gesprächsprotokolle solcher Kontakte finden sich in CIA-Quellen. Siehe z.B. CIA Information Report, 30.6.55, CREST: \#RDP83*004-8; die Ġašğā i-Stammesführer im amerikanischen Exil buhlten weiterhin um amerikanische Unterstützung gegen den Schah. Bowling in Teheran zufolge waren die Khane inzwischen für den Schah ,, as dangerous as the Sioux Indian chiefs are to the U.S."; Bowling Letter, 12.5.59, ,Official-Informal Letters from Tehran 1959‘, Lot 61 D 407, Suppl. Subject Files Relating to Iran, 1956-59, OiCIA/GTI, Box 21; vgl. ed. note, FRUS 58-60, XII, S. 625f.; offenkundig versuchten Teile der Opposition, den USA ihre eigene grundsätzlich prowestliche Orientierung zu signalisieren, um sich als glaubwürdige Alternative zum Schah zu positionieren. So veröffentlichten einige oppositionelle Nationalisten in der Zeitung Ettelā'āt eine Anzeige in Unterstützung der Eisenhower-Doktrin. ,There is no evidence whatsoever that either they im Dokument werden ,some of the innumerable American intelligence personnel here " erwähnt; R.P.\} or the American Embassy proper are running the Nationalist Resistance Movement group as a shadow alternative government", berichtete die britische Botschaft ans Foreign Office. Vgl. Stevens Letter, 2.3.57, ,Iran Official-Informal Letters from Tehran 1957`, Lot 60 D 533, Subject Files Relating to Iran, 1951-1958, OiCIA/GTI, Box 9; Russell to FO, No. 126, 6.11.57, FO 371/127075; Die britische Botschaft vermutete Unterstützung Baxtijārs für die Aktion gegen die Politik des Schahs. Letzterer ,has seized - some would say actually created - the opportunity at once to crack down on the Iran party and to demonstrate to the Americans that he disapproves of their alleged flirtations with it."; Stevens to Lloyd, No. 24, 9.2.57, FO 371/127074, TNA:PRO.

279 Vgl. State \#1726, 28.2.58, FRUS 58-60, XII, S. 541f.; Pa10/14/58, Stevens to Riches, 4.3.58, FO 371/13309, TNA:PRO; umfangreiche Recherchen bei GASIOROWSKI, Qarani Affair; zu Garani vgl. MiLANI, Eminent Persians: I, S. 445-50.

${ }^{280}$ Bereits Anfang 1957 berichtete die Botschaft, dass Garani in einer „excellent position for conspiracy“ sei. Er sei zudem der einzige hohe Offizier ,who holds pro-Mosadeq Nationalist sympathies.“; Tehran \#709, 12.2.57, 788.5/2-1257; Vgl. Tehran \#1663, 2.3.58, 788.00/3-258, CF 55-59; allerdings waren weder Kontakte zur NWI noch zur Tudeh nachgewiesen worden. Dem CIA Station Chief zufolge hielt sich Garani wohl für eine Art iranischen Nasser (,,rather a ludicrous image that he had of himself"); Gratian Yatsevitch OHI, 5.11.88, OHIC, S. I-39; Ein westdeutscher Diplomat sprach vom ,Zwischenfall der amerikanischen Mitwisserschaft, um nicht zu 
offenbar eine Zeitlang von einer Involvierung der USA überzeugt, da Ġarani 1956 mehrere Monate zu Trainingszwecken in den USA verbracht hatte und sich zahlreiche Kontakte der Verschwörer zu amerikanischen Offiziellen nachweisen ließen und darüber hinaus einige der Beteiligten Teil des pro-amerikanischen Netzwerks in Iran waren. Der von den iranischen Behörden implizierte 'Ali Amini stand der politischen Gruppe Garanis nahe, war aber allem Anschein nach nicht in die Coup-Planungen eingeweiht gewesen. Aminis Ruf bleib dennoch beschädigt und er wurde später als Botschafter in Washington abberufen, in erster Linie, da er ,personally disliked and feared by $\{$ the; R.P. $\}$ Shah“ sei. ${ }^{281}$ Im Zuge der Aufklärung der Affäre stellte sich jedoch heraus, dass Ġarani und seine Unterstützer autonom gehandelt und sich zudem vergeblich um Unterstützung der USA bemüht hatten. ${ }^{282}$ Trotz der Aufklärung blieb anschließend ein Reservoir an Misstrauen, zumal der Schah die amerikanischen Kontakte mit iranischen Oppositionellen im Kern als zweifelhaft betrachtete und durchaus zurecht Vorwissen der CIA über die Coup-Absichten vermutete und sich daher fragen musste, warum er nicht im Vorfeld von dieser Seite Warnungen vor dem beabsichtigten Staatsstreich erhalten hatte. ${ }^{283}$ Möglicherweise blieben den USA weiter reichende Folgen aus der Affäre erspart, da der Schah zu dieser Zeit abgelenkt war durch persönliche Belange: Im März 1958 wurde die Scheidung seiner kinderlos gebliebenen Ehe mit seiner zweiten Gattin Sorajā bekanntgegeben - er

sagen Förderung, der Umsturzpläne ehrgeiziger iranischer Militärs und Politiker"; Botschaft Teheran an AA, 8.5.55, B 12/1004, PAAA.

281 Tehran \#1663, 2.3.58, 788.00/3-258, CF 55-59; eindeutiger war wohl die Involvierung des späteren Landwirtschaftsministers Hasan Arsanğāni im Kabinett Amini von 1961. Vgl. Tehran \#1748, 15.3.58, 788.00/31558; Tehran \#1716, 11.3.58, 788.00/3-1158; Tehran \#1647, 28.2.58, 788.00/2-2858, CF 55-59; offenkundig gingen die US-Kontakte zu den Verschwörern auf deren Initiative zurück. In der Retrospektive mussten die zahlreichen Verbindungen zu pro-amerikanischen Gewährsleuten in Iran wie dem ehemaligen CIA-Zuarbeiter Esfandijār Bozorgmehr aus Sicht des Schahs dubios erscheinen. U.A. zu Bozorgmehrs Treffen mit Rountree in Athen vgl. Tehran \#721, 10.2.58, FRUS 58-60, XII, S. 537ff.; zu Bozorgmehrs CIA-Verbindungen vgl. GASIOROWSKI, Qarani Affair, S. 632; COTTAM, Nationalism in Iran, S. 298f., möglicherweise selbst damals als CIA-Agent in die Affäre involviert, deutet jedoch eine Beteiligung Aminis an. Zu Cottams Involvierung KATOUZIAN, Struggle for Power, S. 214f.

${ }_{282}$ Eine vermutlich sowjetische Fälschung eines angeblichen US-Telegramms, das herablassende Bemerkungen über den Schah enthielt, half der Botschaft in ihrer Rehabilitierung. Mohammad Rezā glaubte von nun an eine Verschwörung mit dem Ziel, seine Beziehungen zu Washington zu verschlechtern. Vgl. Tehran \#1657, 1.3.58, 788.00/3-158, CF 55-59; Tehran \#1663, 2.3.58, 788.00/3-258; zur sowjetischen Rolle vgl. ANDREW/MITROKHIN, The Mitrokhin Archive II, S. 170f.; bizarr die Verwendung der Fälschung und die Darstellung der Affäre als teilweise von islamistischen Schläferzellen ausgeführt bei MILANI, The Shah, 206-12.

${ }_{283}$ Am plausibelsten erscheint die Erklärung, dass sich die Putschisten durch Kontakte mit reformistisch eingestellten US-Offiziellen niedrigeren Ranges ermuntert fühlten und ganz bewusst selbst den Eindruck amerikanischer Sanktionierung erwecken wollten, um die eigenen Erfolgschancen zu erhöhen. Im Februar 1958 war die offizielle US-Politik weiterhin bestimmt durch NSC 5703/1 und somit noch offiziell bestimmt durch weitgehend bedingungslose Unterstützung Mohammad Rezās. Eine ähnliche Interpretation bei BILL, Eagle and Lion, S. 127f.; auf Anfrage des NSC hatte der Planungsstab im State Department im Sommer 1959 eine Studie über die Implikationen der Machtübernahme in peripheren Staaten durch die Streitkräfte im Hinblick auf strategische Interessen der USA unternommen. Die Studie, maßgeblich verfasst von Henry Ramsey, konstatierte, man sei „unrealistic in expecting that the sophisticated norms of democracy could work, either politically or economically, once Free Asia cut its colonial ties and accepted the full responsibilities of government."; im Kern begrüßte die Studie das Aufkommen von Militärregierungen als potentiell ,imposing stability and decisiveness“" und empfahl amerikanische Unterstützung für solche Regime. Siehe Policy Planning Staff Long Range Planning Paper No 1959-6/1, ,Political Implications of Afro-Asian Military Takeovers', 18.6.59, S/P Papers, June 1959", Lot 67 D 548, S/P Records: 1957-1961, Box 196, RG 59, NACP; Eisenhower selbst bezeichnete Ramseys Studie als den ,finest report“, der jemals im NSC vorgestellt worden war. DCI Allen Dulles betonte die Wichtigkeit der Herstellung von ,appropriate relationships with the rising military leaders and factions" mittels der MAAGs, eine relativ unverhohlene Forderung nach geheimdienstlicher Nutzung der Militärmissionen, hier wieder stark unterstützt vom Präsidenten, und betonte ein weiteres Mal den grundlegenden Schock der Irakischen Revolution. Vgl. 410th NSC Meeting, 18.6.59, FRUS 58-60, XVI, S. 97f., 99; die Debatte in dieser NSC-Sitzung ist zentral zum Verständnis der Eisenhower-Politik gegenüber den neuen unabhängigen Staaten. 
heiratete schließlich Ende 1959 Farah Dibā und im folgenden Jahr wurde ein Thronfolger geboren, was die Unsicherheit über die unmittelbare Nachfolge beseitigte. ${ }^{284}$ Offenbar hatten die Amerikaner auch für den Fall der Ausweisung von US-Offiziellen mit Konsequenzen hinsichtlich der Wirtschaftshilfe gedroht. Die Ġarani-Affäre belastete jedoch Mohammad Rezās Verhältnis zur amerikanischen Schutzmacht und insbesondere zu Botschafter Chapin, der anschließend durch Edward T. Wailes ersetzt wurde. Zudem untersagte Secretary Dulles die Fortsetzung von Kontakten amerikanischer Offizieller mit Dissidenten, um eine Wiederholung eines solchen Vorfalls auszuschließen. ${ }^{285}$ So informierte CIA-Direktor Allen Dulles im Juli 1959 den NSC, man habe Kontaktversuche iranischer Oppositionsführer „rebuffed“. 286

Ein weiterer Faktor, der zur Entstehung der bilateralen Krise von 1959 beitrug, war eine präzedenzlose Uneinigkeit in Ölfragen. Die Aushandlung des Konsortiums war ein Meisterstück amerikanischer Stabilisierungspolitik in Iran gewesen und hatte einen entscheidenden Beitrag zur Durchsetzung einer präponderanten Stellung der USA in dem Land geleistet. Mohammad Rezā begann jedoch bald, seine Unzufriedenheit mit Irans Position gegenüber dem Konsortium zu äußern. Als zu langsam befand er die Steigerung des iranischen Marktanteils und damit der Öleinnahmen, die zentral für seine Aufrüstungsbestrebungen waren. Zudem waren unabhängige Bestrebungen in der Rohstofffrage ein traditionelles Spielfeld des iranischen Nationalismus und zentrales Element der einsetzenden Mythologisierung Mosaddeġs in der Erinnerungskultur Irans. ${ }^{287}$ Mohammad Rezā erhoffte sich von einer Konfrontation mit den Öl-Majors mehr Popularität, als er Anfang 1957 gegen den Rat der meisten Kabinettsmitglieder ein Gesetz zur Erschließung von Ölfeldern in Gebieten außerhalb der Konsortiumsvereinbarung in den Mağles einbrachte, das eine Abkehr von der 50:50Profitteilungsformel implizierte und zudem einen weitaus größere Kontrolle des Förderlandes auf Managemententscheidungen und Teilhabe an der Wertschöpfung in Weiterverarbeitung

${ }^{284}$ Vgl. Milani, The Shah, S. 212-9; vgl. Tehran \#233, 3.11.60, 788.11/11-360, CF 60-63.

${ }^{285}$ FARDUST, Rise and Fall, S. 164f., behauptet, der Schah sei vom MI6 gewarnt worden. Zu Dulles' Weisung siehe FRUS 58-60, XII, S. 582 n2; selbst dem Schah ging dies wohl zu weit. Siehe Tehran \#304, 1.8.58, ebd., S. 582f.; Chapin verteidigte die Notwendigkeit solcher Kontakte. Vgl. Chapin Letter, 2.6.58, ebd., S. 552f.; die rasche Aufdeckung schreckte vermutlich auch zukünftige Coup-Planer ab: „The failure of the alleged plot of General Gharani in 1958 has sometimes been attributed to foreign knowledge of its existence and this attribution will have increased the propensity of any serious military plotters to avoid foreign contacts."; Tehran \#A-128, 27.8.63, DEF 6 IRAN, CF 63; die Briten verteidigten ebenfalls die Notwendigkeit solcher Kontakte. Vgl. Pa10/14/58, Stevens to Riches, 4.3.58, FO 371/13309, TNA:PRO; zu Chapin vgl. Botschaft Teheran an AA, 22.10.57, B 12/1005, PAAA; noch 1960 wurde die Garani-Gruppe weiterhin als die stärkste und kohärenteste Widerstandsgruppe unter den Offizieren eingeschätzt. Vgl. Marcy Memo, 19.7.60, ,14 Opposition Movements, 1960`, NEA, GTI: RIAD, 1958-63, Box 2; vgl. GASiOROWSKI, Qarani Affair, S. 638ff., sieht die Affäre als Auslöser der begrenzten Reformschritte des Schahs im Anschluss und auch eines weiteren Professionalisierungsschubs der Sicherheitsorgane, nunmehr in engerer Kooperation mit dem Mossad, auch um den US-Einfluss zu reduzieren. Garani wurde nach der Revolution von 1979 für kurze Zeit Stabschef der iranischen Armee und im April 1979 durch die mysteriöse Forgāān-Gruppe ermordet. Vgl. MiLANI, Eminent Persians: I, S. 445-51; interessant sind die vielen Parallelen der Ġarani-Affäre zu den Vorgängen in einem anderen amerikanischen Klientelstaat, dem missglückten Staatsstreich gegen Kaiser Haile Selassie im Dezember 1960 in Äthiopien. Vgl. MARCUS, Ethiopia, S. 116-49.

${ }^{286}$ Zit. aus ed. note, FRUS 58-60, XII, S. 645; eine weitere Konsequenz dieser Zurückhaltung war ein begrenzter Informationsstand über eventuelle Coup-Planungen in Iran infolge der ,sensitivity“ Mohammad Rezās. Vgl. Briefing for Planning Board, 9.10.58, ,Iran (2)‘, WHO, NSCS, Special Staff File Ser., Box 4, DDEL.

${ }^{287}$ Vgl. KaUZ, Perception; SiAvoshI, Oil Nationalization Movement. 
und Vertrieb durchsetzte. Trotz scharfer amerikanischer Proteste beharrte der Schah auf dem eingeschlagenen Kurs und die NIOC schloss mit der italienischen Gesellschaft Agip im August 1957 die sogenannte SIRIP-Vereinbarung ab, die neuartige Vertragsformen und eine de-facto 75:25-Profitteilung zugunsten des Förderlandes beinhaltete. „This represents a significant and radical change from the pattern now prevalent throughout the Middle East [...].“288 Es folgte im Juni 1958 eine ähnliche Vereinbarung mit einer Tochterfirma der Standard Oil Company of Indiana, die auf dem Papier sogar noch vorteilhafter für Iran war. ${ }^{289}$ All diese Entwicklungen waren Anzeichen für eine langsame Erosion der tradierten Kontrolle der nahöstlichen Ölförderung und -vermarktung durch westliche Konzerne sowie der absehbaren Infragestellung der 50:50-Formel und der stärkeren Beteiligung der Förderländer am Management der Ölunternehmen. ${ }^{290}$ Die Gründung der Organization of the Petroleum Exporting Countries (OPEC) im September 1960 durch vier nahöstliche Förderländer, darunter Iran, sowie Venezuela, signalisierte die Bereitschaft dieser Staaten mittels eines Förderkartells auf Preiserhöhungen und stärkeren Einfluss hinzuarbeiten. ${ }^{291}$

Neben einer unabhängigen Ölpolitik glaubte sich Mohammad Rezā zudem mittels einer selbstbewussteren Außenpolitik im nationalen Sinne profilieren zu können. Unmittelbar nach der Suezkrise hatten Saudi-Arabien und Iran die kleinen unbewohnten Inseln Arabi und Farsi im Persischen Golf besetzt, dabei die Handlungsunfähigkeit Großbritanniens ausnutzend. Im

\footnotetext{
${ }^{288}$ Cumming Memo, 20.8.57, FRUS 55-57, XII, S. 937; vgl. Tehran \#1086, 17.1.57; State \#1401, 26.1.57; State \#1940, 9.4.57, ebd., S. 873, 883, 932f.; stellvertretend für die besorgte Haltung der Majors Page Letter, 1.5.56, ebd., S. 815f.; die italienische Regierung erschien aus Sicht der amerikanischen Öffentlichkeit als ,irresponsible opportunist unmindful of international responsibilities“; Rome \#3702, 18.3.57, ebd., S. 920; Agip (Azienda Generale Italiana Petroli) war die upstream-Tochter des staatlichen Mineralölkonzerns Eni (Ente Nazionale Idrocarburi) - SIRIP stand für die neugegründete gemeinsame Gesellschaft Société Irano-Italienne des Pétroles. Während die US-Offiziellen gegenüber Italienern und Iranern die mangelnde Wirtschaftlichkeit der Vereinbarung betonten, glaubte der OCB, ,[...] chances for success are not inconsiderable and might lead in time to the Italian agency becoming an important factor in Iranian oil production.“; OCB Progress Report, 21.8.57, ,NSC 5703/1", Policy Papers, Box 43, RG 273, NACP; vgl. Stevens to Lloyd, No. 47, 12.4.57, Teil von Ministry of Power Study for Macmillan, 6.5.57, PREM 11/2032; die Briten empfahlen eine pragmatische Reaktion angesichts der Entschlossenheit der Italiener und Matteis ,to muscle in on Middle East oil“". Die Majors und die US-Regierung „,would be wise to consider whether it might not be a lesser evil to find room for the Italians that to give them cause to run amok in the Middle East.“; O.M.E.(57)24 vom 25.3.57, CAB 134/2339, TNA:PRO; vgl. auch Parviz MINA, „Oil Agreements in Iran“, in: Encyclopeedia Iranica, online edition, New York, 1996-, verfügbar auf http://www.iranicaonline.org/articles/oil-agreements-in-iran; vgl. FARMANFARMAIAN, Blood and Oil, S. 320; zu Matteis Operationen vgl. auch PozZI, Case of ENI, S. 267; TREMOLAdA, Enrico Mattei in Iran, betont Matteis Revanchegelüste aufgrund seiner Nichtbeteiligung am Konsortium. Vgl. auch COLIAEI-KAWKA, Relations officielles.

${ }^{289}$ Der Schah bezeichnete diese Vereinbarung als wichtigste Errungenschaft seit der Restitution Aserbaidschans. Der Konzern sagte Explorationen im Golf in einer Größenordnung von \$82 Millionen zu und zahlte einen VorabBonus von \$25 Millionen. Die von Mohammad Rezā erwarteten jährlichen Einnahmen über eine halbe Milliarde Dollar innerhalb von 5 Jahren stellten sich aber nicht ein. Vgl. OCB Progress Report, 8.10.58, ,NSC 5703/1 ${ }^{\circ}$, Policy Papers, Box 43, RG 273, NACP; vgl. FERRIER, Iranian Oil Industry, S. $116 \mathrm{ff}$.

${ }_{290}$ Befürchtet wurde zuvorderst eine Politisierung der Ölfrage. So warnte die US-Botschaft, Mohammad Rezā könnte auf die Verweigerung von Budgetzuschüssen durch Washington mit einer Revision der Konsortiumsformel zugunsten einer 75:25-Profitteilung reagieren: ,This change would just about balance the Iranian budget without assistance from us [...].“; gleichzeitig begannen die Iraner nun mit Nachdruck auf eine Steigerung der Ölförderung in Iran zu drängen. Wailes Letter, 15.1.59, ,Correspondence with Ambassador, 1959`, Lot 61 D 407, Suppl. Subject Files Relating to Iran, 1956-59, OiCIA/GTI, Box 20; vgl. Stillman Memo, 18.12.59, ,Iran, Consortium 1959‘, ebd. ${ }^{291}$ Angesichts der globalen Produktionsüberschüsse war der Ölmarkt allerdings weiterhin ein ,Buyer's Market “ und die politische Gestaltungskraft des Kartells zu diesem Zeitpunkt überschaubar, was sich allerdings in den 1970ern dramatisch ändern sollte. Vgl. CIA, NIE 30-60, 13.12.60, FRUS 58-60, IV, S. 665-79; Martin Memo, 1.4.60, ,US-UK Middle East Oil Concessions Talks, 1959-60‘, NEA/NE: Records of the Officer in Charge of Economic Affairs, 1947-1963, Box 5, RG 59, NACP; zum regionalen Kontext der OPEC-Gründung vgl. CITINO, Arab Nationalism to OPEC, S. 145-60.
} 
Anschluss vertrieben iranische Kräfte die Saudis auch von Arabi, damit den Wettbewerb um die Kontrolle einer Reihe von Inseln im Golf einleitend, der 1971 mit der iranischen Besetzung der strategisch wichtigsten Inseln Abu Musā sowie der Großen und der Kleinen Tunb-Inseln nahe der Straße von Hormus seinen Höhepunkt erreichen sollte, bis zur Gegenwart Anlass für Spannungen zwischen Iran und den arabischen Anrainerstaaten. ${ }^{292}$ Die Kontrolle über die Inseln war potentiell bedeutend für zukünftige Ansprüche auf Offshore-Ölvorkommen. Der Konflikt um die beiden Inseln wurde während Mohammad Rezās Staatsbesuch in SaudiArabien im Frühling 1957 rasch geschlichtet, doch die Eisenhower-Administration erkannte die möglichen Folgen eines iranischen Expansionismus im Persischen Golf, zumal Iran nun aktiv die Scheichs in den britisch kontrollierten Protektoraten in Katar und in der Vertragsküste hofierte und der Schah im November eine Gesetzesvorlage im Mağles lancierte, welche die Ansprüche auf Bahrain wiederbelebte. ${ }^{293}$ Der iranische Aktivismus und die Gebietsansprüche im Persischen Golf hatten bereits vor der Revolution die Beziehungen zum damals noch verbündeten Irak belastet - nach dem Juli 1958 behandelte der Schah die neue nationalistische Führung in Bagdad durchweg als sowjetischen Satelliten und Bedrohung für Iran. Teheran unterstützte anschließend die Idee einer monarchischen Restauration in Irak und forderte mehrfach amerikanische Unterstützung für diese Pläne. ${ }^{294}$ Im Herbst 1959 provozierte die iranische Regierung sodann ein Wiederaufflammen des alten Konflikts mit Irak um die Šațț al'Arab-Frage. ${ }^{295}$

Das neue Selbstbewusstsein des Schahs im Hinblick auf eine unabhängige Ölpolitik und auf die eigenen Ambitionen für eine zukünftige Rolle als Ordnungsmacht im Persischen Golf

\footnotetext{
${ }^{292}$ Offenbar war anfangs die Besetzung dieser Inseln sowie der Insel Sirri ebenfalls für diesen Zeitpunkt geplant gewesen. Das State Department verweigerte Involvierung in die territorialen Konflikte um die Inseln und warnte Iran vor einer Instrumentalisierung nationalistischer Leidenschaften. Vgl. Kuwait \#90, 16.10.56, 788.022/10-1656; Tehran \#560, 16.10.56, 788.022/10-1356; State \#851, 1.11.56, 788.022/10-2456, CF 55-59.

${ }^{293}$ Mohammad Rezā stimmte einer Räumung Arabis zu, dass anschließend wieder von den Saudis besetzt wurde. London protestierte vergeblich in beiden Hauptstädten. Die Macmillan-Regierung bat zudem die USA um Unterstützung gegen die iranischen Ansprüche auf Bahrain. Vgl. Rountree Letter, 24.8.57; Staff Summary Supplement, 17.12.57, FRUS 55-57, XII, S. 939f., 961; vgl. ebd., S. 939 n3; NSC 5821/1, [13.11.58], FRUS 5860, XII, S. 608; MemCon, 29.3.57, 788.022/3-2957; Tehran \#470, 23.11.57, 788.022/11-2357, CF 55-59; Loy Henderson empfahl den Briten: ,[...] we must press the Shah to let the Persian claim to Bahrain die, if we are to avoid real trouble on this in a few years' time. We can certainly encourage American pressure on the Shah to this end.“; Memo for Macmillan, 28.6.57, PREM 11/1937; vgl. FO Minute, 24.2.56, FO 371/120595; Russell to Lloyd, No. 89, FO 371/126909; Stevens antwortete einem iranischen Offiziellen: „Chronologically the Iranian claim seemed to be about on a par with a British claim to the United States."; Stevens Letter, 12.3.57, FO 371/126930; erste Überlegungen eines Inseltausches gab es bereits 1955. Vgl. Lloyd Memo, 31.1.55, FO 371/114640, TNA:PRO.

${ }^{294}$ Vgl. Tehran \#729, 13.4.59, 687.88/4-1359, CF 55-59; Stevens to FO, No. 575, 21.7.58, FO 371/134210, TNA:PRO; vgl. POPP, Working Relationship, S. 410f.; SAMII, Lebanon Policy, S. 66-9; die neue Führung in Bagdad provozierte mit der Annahme einer neuen politisch motivierten Sprachregelung und wechselte von „Persischer Golf“ auf „Arabischer Golf“, einen bis heute andauernden Disput anstoßend. Vgl. Botschaft Teheran an AA, 12.8 .55 , B 12/1007, PAAA.

${ }^{295}$ Für eine Weile befürchteten die USA in der Tat eine militärische Konfrontation bzw. eine Aufkündigung des Grenzvertrags von 1937 durch den Schah. Vgl. Tehran \#383, 19.12.59, 687.883/12-1959, CF 55-59; Basra \#31, 25.8.60, 988.734/8-2560, CF 60-63; vgl. CIA Memo RR GM 60-61, 7.1.60, CREST: \#RDP79*001-0; Botschaft Teheran an AA, 22.10.59, B 12/1006, PAAA; gleichzeitig begann Teheran Propaganda- und Subversionsaktivitäten gerichtet auf die irakischen Kurden. Zudem involvierten die Iraner die USA in ihre Propagandaaktivitäten gegen Bagdad mittels des Counter-Subversion Committee der CENTO. Einige Mitglieder der Exil-Tudeh fanden zudem in Bagdad Zuflucht. Vgl. Walstrom Memo, 30.9.60, ,1960 Chron.-Inter-Office Memos (1 of 2)', Records of the Director (NE): 1958-1963, Box 1, RG 59, NACP; Harrison to Lloyd, No. 158, 4.12.58; Chancery Tehran to Eastern Department, 19.12.58, FO 371/133083, TNA:PRO.
} 
machte sich bereits vor der Krise von 1959 in den Beziehungen zu Washington bemerkbar. Seit Mitte der 1950er Jahre bemühte sich Mohammad Rezā, Iran aus der einseitigen Abhängigkeit von den USA zu lösen und die Außenbeziehungen zu diversifizieren. In wirtschaftlicher Hinsicht nahm der Handel mit Westeuropa und insbesondere mit der Bundesrepublik Deutschland erheblich an Bedeutung zu. ${ }^{296}$ Außenpolitisch war die Suche nach alternativen Partnern aber weitaus schwieriger. Ein offenkundiger Kandidat war Großbritannien, traditionelles Bête Noire des iranischen Nationalismus. Die USA bescheinigten der ehemaligen britischen Vormacht Ende 1958 weiterhin ,a considerable measure of influence in Iran“ und Mohammad Rezā versuchte mehrfach, allerdings weitgehend vergeblich, die AngloAmerikaner gegeneinander auszuspielen. ${ }^{297}$ Im Sommer 1957 regten die Iraner Gespräche mit London über die Zukunft des Persischen Golfes an und deuteten in diesem Kontext auch eine neue Bereitschaft an, Großbritanniens gegenwärtige Ordnungsrolle dort anzuerkennen. Die Hoffnungen auf eine strategische Partnerschaft erfüllten sich nicht. ${ }^{298}$ In London begrüßte man die Annäherung an Iran, zumal angesichts der angespannten Beziehungen zu den anderen beiden ,großen“ Anrainerstaaten Saudi-Arabien und Irak, doch hatte man auf die Ereignisse von 1958 mit einer Aufgabe der traditionellen Rolle als Ordnungsmacht - ,[...] to escape from the position of backing one part of the Arab world against the other and thus appearing to be the cause of its division“ ${ }^{299}$ - und mit einer noch stärkeren Fokussierung der Nahoststrategie auf die Überreste der eigenen Position im Golf und in Südarabien reagiert. Zentral waren nun die kleinen Golfscheichtümer und vor allem das „Golden Nugget“ Kuwait - Großbritannien war daher zu keinerlei Zugeständnissen bzw. zu einer Machtteilung im Golf bereit und setzte stattdessen weiterhin voll auf Unterstützung Washingtons für die Fortsetzung der informellen britischen Kontrolle dort. ${ }^{300}$ Die britische Alternative war somit keine wirkliche - wenigstens

\footnotetext{
${ }^{296} 1959$ konstatierte man in GTI, dass Westeuropa und insbesondere Westdeutschland eine ,,dominating position among Iran's suppliers“ erlangt habe. Vgl. Merriam Memo, 22.7.59, ,Iran Imports + Exports (Trade) 1959 6-C“, Lot 61 D 407, Suppl. Subject Files Relating to Iran, 1956-59, OiCIA/GTI, Box 21.

${ }^{297}$ NSC 5821/1, S. 608; so drohte er bereits im Februar 1955 damit, London um Militärhilfe anzugehen. Vgl. FRUS 55-57, XII, S. 702 n2; Tehran \#641, 30.1.57, 641.88/1-3057, CF 55-59; der britische Botschafter Stevens verwahrte sich Ende 1956 gegen Behauptungen im Nachgang von Suez, man sei von den USA aus Iran vertrieben worden. Stattdessen sei die Wiederherstellung der diplomatischen Beziehungen und die (Teil-)Rückkehr in die iranische Ölproduktion ,the fruit of long and patient U.S. diplomatic and planning activity for which we have every reason to be deeply grateful."; Stevens to Lloyd, No. 139, 7.12.56, FO 371/120724, TNA:PRO.

298 „Realising Britain's vital need for Middle Eastern oil, the Iranian Government was now prepared to recognise that it was in the common interest to preserve the British 'special position' along the strategic oil-route."; Russell to Lloyd, No. 98, 24.8.57, FO 371/126909; die Macmillan-Regierung betrachtete die Position im Golf als ,probably the one remaining vital asset in the Middle East which we can at least come within measurable distance of protecting on our own" und lehnte Überlegungen einer Anpassung der Beziehungen zu den Scheichtümern an die zu erwartende zukünftige Erosion des eigenen Einflusses in der Region strikt ab. Vgl. O.M.E. (57) 31, 2.2.57, CAB 134/2339, TNA:PRO.

${ }_{299}$ O.M.E. (58) 45, 15.10.58, CAB 134/2342; vgl. M.E. (M)(59), 1st Meeting, 16.1.59, CAB 134/2230; M.E.(M)(59) 3rd Meeting, 9.2.59, CAB 134/2230; FO Paper, ,Points for a Middle East Policy: Part II‘, o.D., CAB 21/3935; TNA:PRO.

${ }^{300}$ Zentral war aus britischer Sicht zu verhindern, dass „Arab nations control completely all the sources of Arab oil they can hold us to ransom [...]“. Sollte sich der Herrscher von Kuwait der VAR annähern, ,,we are faced with the dilemma of either deposing him and more or less occupying and running Kuwait as a colony, or acquiescing in the loss of the remaining most important source of Middle Eastern oil.“; GEN. 658/1st Meeting, 22 Jul. 1958, CAB 130/153; , golden nugget" in Arabian Department Background Paper, 22.4.63, T 312/550; ,,Kuwait, with its massive oil production, is the key to the economic life of Britain - and of Europe."; Eintrag vom 18.7.58, in: CATTERAll, Macmillan Diaries: I, S. 137; vgl. GALPERn, Sterling, S. 198-244; ein US-Diplomat berichtete, die Briten, show some reserve in sharing inside information with Americans, which in view of the fact that Kuwait is perhaps the cornerstone of British policy in the Arab world is understandable."; Meyer Report, 17.6.59, ,Kuwait",
} 
gelang es, zumindest symbolisch das amerikanische Militärmonopol in Frage zu stellen, mittels einer Zusage Londons für ein limitiertes Trainingsprogramm für die iranische Marine. ${ }^{301}$

Positiv wirkte sich die geänderte Herangehensweise der Briten mit Blick auf die iranischen inneren Verhältnisse aus. Zwar sah die Macmillan-Regierung die politische Lage in Iran durchaus ähnlich wie der amerikanische Verbündete, hielt aber an einer Politik der ,noninterference“ in die inneren Angelegenheiten fest. Stabilität habe Priorität vor den ,luxuries of democracy“ - das Land sei noch keineswegs reif für Demokratie nach westlichem Vorbild, rechtfertigte sich der Chargé in der Teheraner Botschaft: „At the moment the average Iranian's political maturity is about equal to the average Tehran taxi-driver's traffic sense - and road manners. A country which is only now having its industrial revolution cannot be thrust overnight into the mould of Westminster." Anders als die Eisenhower-Administration wollten die Briten jeden Eindruck eines Abrückens von der Unterstützung für den Schah vermeiden. ${ }^{302}$ Nach der Ġarani-Affäre bedankten sich die Iraner explizit für die eindeutige Haltung Großbritanniens, offenkundig als Kritik an den Amerikanern zu verstehen. ${ }^{303}$ Trotz dieser neuen Gemeinsamkeiten entstand keine Neuauflage der engen bilateralen Beziehungen der Vergangenheit. Zu schwerwiegend waren die Belastungen, die aus der Geschichte herrührten. John W. Bowling, bald einflussreichster Iranexperte im State Department, zu dieser Zeit noch Erster Sekretär in der Teheraner Botschaft, konstatierte Ende 1956: „Fear and distrust of the British reaches the proportion of an epidemic mental disease in Iran.“ ${ }^{\text {304 }}$

Lot 61 D 43, Office Files Relating to Middle Eastern Affairs, 1958-1959, NEA/NE, Box 15; die Briten versuchten vergeblich 1958 und ein weiteres Mal 1959 substantielle ,joint contingency planning“ für den Persischen Golf in die Wege zu leiten, doch versandeten diese Initiativen aufgrund amerikanischen Desinteresses. Vgl. FO to Embassy Washington, No. 5366, 31.7.58; Dulles Letter, 3.8.58, PREM 11/2399; JP (58) 174 (Final), 25.2.59, DEFE 6/52, TNA:PRO; Dennison Memo, 29.6.59 MemCon, 28.8.59, FRUS 58-60, XII, S. 224-6, 233-5; Macmillan to Eisenhower, 14.5.59; Eisenhower to Macmillan, 15.5.59, in: GEELHOED/EDMONDS, MacmillanEisenhower Correspondence, S. 255-6; vgl. London \#3397, 29.11.57, ,General/British Position in Persian Gulf...', Lot 61 D 48, Records Relating to the Arabian Peninsula, 1948-1959, NEA/NE, OiCIA, Arabian Peninsula Affairs, Box 15; einige der aus der US-UK Agreed Minute vom 23.3.59 hervorgegangenen Studien bez. des Iraks, des Irans und Kuwaits in ,Near and Middle East 1959-61', Lot 67 D 548, S/P Records: 1957-1961, Box 154, RG 59, NACP; vgl. auch FAIN, Ascendance and Retreat, S.77-110; SMITH, Revival and Fall, S. 116ff.; ders., Ending Empire, S. 86-91; vgl. unten Kapitel V.

${ }^{301}$ In diesen Kontext gehörten auch die Drohungen des Schahs, eine Revision der der ARMISH-Vereinbarung und die Streichung des Artikels 24 anzustreben. Das Programm fand im Rahmen des Bagdadpaktes statt und sah die Ausbildung einiger iranischer Marineoffiziere in Großbritannien und gemeinsame Manöver im Golf vor. Das Bekanntwerden der Existenz einer von den Briten betriebenen Ausbildungsstätte für Kampfschwimmer hatte im September 1957 einen amerikanischen Protest wegen Verletzung des Art. 24 zur Folge. Vgl. State \#91, 16.7.56, 788.5/7-1656; State \#2443, 30.9.57, 788.5/9-3057; die JCS empfahlen Anfang 1961 schließlich die Etablierung einer Navy Section im Rahmen von ARMISH-MAAG. Siehe JCS Memo, 26.1.61, ,CCS 9181/5000 Iran (13 Apr 1960)', JCS Central Decimal File 1960, Box 80, RG 218, NACP.

302 ,The Shah remains the most important piece on the board. If he falls, the game is over. [...] If the Shah were to go, it is unlikely in the extreme that his place would be taken by a government with which we could come to terms - certainly not to anything like the terms we now enjoy. There is nothing from our point of view even remotely tolerable which is likely to take over."; Russell to Lloyd, No. 146, 27.10.58, FO 371/133007, TNA:PRO.

${ }^{303}$ Vgl. Pa10/14/58, Stevens to Riches, 4.3.58, FO 371/13309, TNA:PRO; Stevens verteidigte aber die USA gegen den Vorwurf der Involvierung: „It stood to reason that they could have no conceivable interest in bringing about a change of régime when their own position in this country was meshed and dove-tailed into the present Government in all kinds of way: financial, military and administrative."

${ }^{304}$ Bowling Memo, 24.12.56, encl. to Tehran \#641, 30.1.57, 641.88/1-3057, CF 55-59; tatsächlich startete der Schah im Herbst 1958 aus opportunistischen Motiven eine anti-britische Kampagne. Vgl. Tehran \#226, 23.9.58, 788.00/9-2358, CF 55-59; Chancery Tehran Letter, 30.9.58, FO 371/133007, TNA:PRO. 
Mit dem Ziel, seine einseitige Abhängigkeit vom amerikanischen Patron zu reduzieren und in der Hoffnung, im Rückgriff auf bewährte Werkzeuge iranischer Außenpolitik entschloss sich Mohammad Rezā zu einer Öffnung gegenüber dem bislang als Hauptfeind deklarierten nördlichen Nachbarn, der UdSSR. Die Aufgabe der traditionellen iranischen Neutralität hatte dem ohnehin diffizilen Verhältnis neue Belastungen aufgebürdet und John Foster Dulles` späte Bedenken über die möglichen Konsequenzen einer Aufgabe der iranischen Neutralität waren nicht zuletzt eine Folge der heftigen Reaktionen in Moskau gewesen. ${ }^{305}$ Die Sowjetunion versuchte im Anschluss, Teheran zu einer Rückkehr in eine neutrale Position zu bewegen und unterbreitete eine Reihe von Vorschlägen für alternative Garantien der Sicherheit Irans. ${ }^{306}$ Während seines ersten Staatsbesuchs in der UdSSR im Juni/Juli 1956 hatte Chruschtschow weitere Versuche in diese Richtung unternommen, erreichte aber nur eine vage Zusage Mohammad Rezās, Iran werde niemals „Sprungbrett“ für eine Aggression gegen die Sowjetunion werden. ${ }^{307}$ Trotz des Beitritts zum Bagdadpakt setzte die sowjetische Führung die Charmeoffensive gegenüber Teheran fort: 1957 folgten bilaterale Vereinbarungen über offene Grenzfragen und eine aus Sicht Teherans sehr willkommene Regelung der Frage der im Zweiten Weltkrieg gepfändeten Goldreserven. Zudem gab es Vereinbarungen zur Ausweitung des Handels mittels Bartergeschäften, der zivilen Luftfahrt, und diverse attraktive sowjetische Angebote zur Entwicklungszusammenarbeit. ${ }^{308}$ Die sowjetische Absicht, die Westbindung Irans zu vereiteln, war allerdings allzu offensichtlich und Mohammad Rezā ging daher nur begrenzt auf diese Offerten ein. Angesichts der Enttäuschungen über die amerikanischen Hilfsleistungen und -zusagen und die ausbleibende Sicherheitsgarantie entschloss sich der Schah im Januar 1959 aber zur Aufnahme von Verhandlungen mit Moskau über einen Nichtangriffspakt zwischen beiden Ländern, was letztlich eine Rückkehr Irans zu einer neutralen Orientierung implizierte.

\footnotetext{
${ }^{305}$ Dulles hatte befürchtet, Moskau könne den Beitritt als Vorwand nehmen, um zu ,,claim the right to bring troops back into Iran under the treaty of 1921."; Record of Meeting, 9.11.55; auch im Foreign Office registrierte man, dass die Sowjets ,really disliked the Bagdad (sic!) Pact and regarded it as a form of encirclement.“; Extracts [Shuckburgh, Russell], 8.11.55, FO 371/115469, TNA:PRO; vgl. FRUS 55-57, XII, S. $773 f . n 7$.

${ }^{306}$ Molotow unterbreitete Anfang 1956 die Idee einer Viermächtegarantie der iranischen Neutralität als Ersatz für den sowjetisch-iranischen Vertrag von 1927. Siehe State \#1387, 14.2.56, ebd., S. 806f.; vgl. ebd., S. 797 n2; zum Hintergrund dieser Ideen und der Rolle von Sejjed Zijāe ad-Din Tabātabāe i vgl. ALvANDI, Flirting with Neutrality; zu den Verhandlungen vgl. RAMAZANI, Iran's Foreign Policy, S. 295-9.

307 Vgl. Note, encl. to Rountree Memo, 20.7.56, FRUS 55-57, XII, S. 837ff.; ,spring board for launching aggression“ in MemCon, encl. to Stevens Letter, 14.1.57, ebd., S. 869; vgl. ALVANDI, Flirting with Neutrality, S. 422; ZAHEDI, Memoirs, Vol. II, S. 93-104; offenbar hatte Mohammad Rezā bereits im Mai 1955 gegenüber dem sowjetischen Botschafter zugesagt, dass er Stützpunkte ausländischer Mächte und den Transfer fremder Streitkräfte durch iranisches Territorium nicht zulassen werde. Vgl. Stewart Minute, 17.5.55, FO 371/115511, TNA:PRO; der Schah hatte nicht zuletzt auf amerikanischen Drängen hin die Annahme der Einladung über ein Jahr verzögert. Der westdeutsche Botschafter berichtete im Juli 1955, die Einladung selbst habe ,überall grosses $\{$ sic $\}$ Aufsehen" erregt. Vgl. Botschaft Teheran an AA, 18.7.55, B 11/1541; zum Besuch selbst vgl. Botschaft Moskau an AA, 14.7.56, B 12/1008, PAAA.

308 Unter anderem bot Moskau Hilfe bei der Errichtung einer Stahlhütte in Iran an - US-Experten hatten dahingehende iranische Wünsche jahrelang ablehnend beschieden. Die Sowjets boten im Oktober 1956 und ein weiteres Mal im November 1958 praktisch „nahezu unbegrenzte“ wirtschaftliche Hilfsleistungen an. Vgl. ed. note; 299th NSC Meeting, 4.10.56, FRUS 55-57, XII, S. 733, 850f.; Staff Paper, o.D., ,Iran (2)‘, WHO, NSCS, Special Staff File Ser., Box 4, DDEL; Tehran \#1001, 27.11.58, 688.00/11-2758, CF 55-59; bezüglich der wirtschaftlichen Folgen warnte ein GTI-Offizieller, dass , , [...] under conditions of pure free trade the USSR would dominate 80 to 90 percent of the market."; Merriam Memo, 22.7.59, ,Iran Imports + Exports (Trade) 1959 6-C', Lot 61 D 407, Suppl. Subject Files Relating to Iran, 1956-59, OiCIA/GTI, Box 21.
} 
In Washington reagierte man mit einigem Entsetzen auf diese sich abzeichnende symbolische Niederlage im Kontext des Kalten Krieges, zeigte sich aber zugleich erbost über den augenfälligen Versuch Mohammad Rezās mittels dieser Form von „Erpressung“ höhere Hilfszusagen seitens der USA zu erreichen. ${ }^{309}$ Ende Januar 1959 traf eine sowjetische Delegation unter Leitung des stellvertretenden Außenministers Wladimir Semjonow in Teheran ein, um die Details eines Nichtangriffspaktes über den Zeitraum von 30 Jahren zu verhandeln. ${ }^{310}$ Seitens der Eisenhower-Administration war man im Gegensatz zu den britischen und türkischen Verbündeten weiterhin überzeugt, dass der Schah bluffte und eine Aufgabe der engen sicherheitspolitischen Bindungen an den Westen nicht ernsthaft in Betracht zöge. Eine persönliche Botschaft Eisenhowers an Mohammad Rezā verzichtete auf zusätzliche Hilfszusagen und warnte stattdessen explizit vor den nachteiligen Folgen eines Nichtangriffspaktes mit Moskau für die Unabhängigkeit Irans und auch für die ,security and stability of Your Majesty's regime“. ${ }^{311}$ Kurz darauf reiste der britische Verteidigungsminister Duncan Sandys nach Teheran und warnte den Schah persönlich vor den Konsequenzen einer Einigung mit Moskau. ${ }^{312}$ Nun unter erheblichem Druck, versuchte Mohammad Rezā alternativ ein bezüglich des Umfanges der Selbstverpflichtungen begrenztes Protokoll mit den Sowjets abzuschließen, sodass Iran dennoch Mitglied des Bagdadpaktes bleiben und auch die „Bilaterals“ mit den USA unterzeichnen könnte. ${ }^{313}$ Obwohl Moskau den iranischen Forderungen weit entgegen kam - Chruschtschow intervenierte persönlich - und sogar einen

\footnotetext{
${ }^{309}$ John Foster Dulles informierte den NSC am 22.1.59 über die ausufernden iranischen Forderungen nach einer umfassenden Sicherheitsgarantie in den vorausgegangenen Verhandlungen über die anvisierten Bilaterals. Teheran wünsche eine Beistandszusage selbst im Falle eines Angriffes eines nicht-kommunistischen Staates. Das Ausmaß amerikanischer Verärgerung zeigte sich im Kommentar des ehemaligen US-Botschafters in Iran und eigentlich Schah-freundlichen George Allen, inzwischen USIA-Direktor: „It was his belief that it was in our best interests not to go any further to appease the Shah, who [...] was the best blackmailer he knew of."; zit. aus ed. note, FRUS 58-60, XII, S. 626; Eisenhower selbst äußerte sich ähnlich. Siehe ebd., S. 627 n2; tatsächlich hatte Mohammad Rezā bereits 1955 gegenüber dem britischen Botschafter im Anschluss an die sowjetische Einladung zum Staatsbesuch in der UdSSR seine ,splendid position to blackmail us“ notiert. Siehe Stevens to FO, No. 19, 7.7.55, FO 371/114868, TNA:PRO; vgl. CIA, Central Intelligence Bulletin, 2.2.59, CREST: \#RDP79*001-5.

${ }^{310}$ Die Eisenhower-Administration erfuhr aus britischen und türkischen Quellen Details über die Verhandlungen. Bestandteile waren der Verzicht Irans auf den Abschluss der bilateralen Vereinbarung mit den USA im Gegenzug für ein Nichtangriffsabkommen, ,indirekte“ Aggression einschließend, dazu Einstellung der sowjetischen Propagandaaktivitäten gegen die Schahherrschaft und gegenüber der kurdischen Minderheit. Zentral aus iranischer Sicht war darüber hinaus, dass Moskau das in den Artikeln V und VI des sowjetisch-iranischen Freundschaftsvertrages von 1921 enthaltene militärische Interventionsrecht für obsolet erklärte, im Gegenzug zu einer öffentlichen Zusicherung Irans, das eigene Territorium niemals für die Zwecke einer Aggression gegen die UdSSR zur Verfügung zu stellen. Vgl. Rountree Memo, 2.2.59, 611.88/2-259, CF 55-59; offenbar verweigerten die Sowjets aber dieses zentrale Entgegenkommen. Vgl. Alvand, Flirting with Neutrality, S. 428ff.; eine Analyse des Vertrags von 1921 in Aufzeichnung, 10.9.58, B 12/1009, PAAA.

${ }^{311}$ State \#1944 [Eisenhower Letter to Shah], 30.1.59, FRUS 58-60, S. 628; die CIA schlussfolgerte, Mohammad Rezā sei ,[...] unlikely deliberately to divorce Iran from its alliance with the West, as long as the West does not cut him off.“; CIA, SNIE 34-2-59, 3.2.59, ebd., S. 634; auch US-Botschafter Wailes ging weiterhin von „,blackmail“" aus, schloss aber den Abschluss eines Nichtangriffspakts dennoch nicht aus. Um dies abzuwenden, sei eine aufgewertete Sicherheitsgarantie vonnöten bzw. großzügige Budgethilfe über $\$ 70$ Millionen, ,topped off by showy offer of rockets, destroyers, or other hardware“. Wailes sprach sich dennoch gegen weiteres „,appeasement" gegenüber dem Schah aus, da ein Entgegenkommen nur weitere Forderungen und neue Krisen in der Zukunft induzieren würde. Siehe Tehran \#1425, 30.1.59, ebd., XII, S. 630; offenbar hatte der Schah Budgetzuschüsse über \$107 Millionen anstelle der zugesagten \$20 Millionen gefordert. Vgl. Tehran \#1417, 29.1.59, 788.5 MSP/1-2959; State \#1957, 31.1.59, 788.5 MSP/1-3159, CF 55-59.

312 Sandys warnte vor einer Abschwächung der moralischen Verpflichtung zur Verteidigung Irans der westlichen Mächte im Falle eines Abkommens. Siehe ALVANDI, Flirting with Neutrality, S. 431f.

${ }^{313}$ Vgl. Rountree Memo, 9.2.59, FRUS 58-60, XII, S. 635ff.; die Amerikaner zeigten sich konziliant, während die Briten warnten, auch ein solches Protokoll würde von Irans Verbündeten interpretiert,,as qualifying (to put it no higher) Iran's loyalty to the Baghdad Pact and its Western allies“; FO to Embassy Washington, No. 900, 8.2.59, PREM 11/3397, TNA:PRO.
} 
Verbleib Irans im Bagdadpakt akzeptierte, scheiterten die Verhandlungen letztendlich. Am 5. März 1959 unterzeichneten die USA und Iran das executive agreement über militärische Zusammenarbeit, unter anderem basierend auf der Eisenhower-Doktrin, in welchem Washington nun auch vertraglich militärischen Beistand im Falle einer Aggression gegen Iran zusicherte. ${ }^{314}$

Die bilaterale Vereinbarung von 1959 sollte bis zur Revolution die eindeutigste vertragliche Selbstverpflichtung der USA im Hinblick auf die Verteidigung der iranischen Unabhängigkeit und territorialen Integrität bleiben. ${ }^{315}$ Mohammad Rezā verteidigte später seinen kurzlebigen Flirt mit Moskau mit der Notwendigkeit, die Amerikaner zu einer stärkeren Sicherheitsgarantie zu bewegen. Tatsächlich aber waren die Umformulierungen in diesem Kontext eher marginal und zusätzliche Hilfszusagen der Amerikaner recht überschaubar. ${ }^{316}$ Statt die beiden Supermächte gegeneinander auszuspielen und materiell zu profitieren, wie Mohammad Rezā es sich vermutlich erhoffte, hatten die riskanten Verhandlungen mit der UdSSR den Effekt einer weiteren Belastung der Beziehungen sowohl zu Washington als auch zu Moskau. Die Sowjets reagierten auf die Desavouierung mit einer heftigen Propagandakampagne, gerichtet zuvorderst gegen Mohammad Rezā selbst. Sowjetische Radiostationen übertrugen fast 50 Stunden in persischer Sprache pro Woche - im April 1959 begann zudem der von iranischen Exilkommunisten geführte, geheime Propagandasender Pejk-e Irān (Stimme Irans) von Sowjetisch-Aserbaidschan aus tägliche Sendungen. Zeitweilig kursierten Gerüchte, die UdSSR könne angesichts der Unterzeichnung der Bilaterals sogar eine neuerliche Besetzung IranischAserbaidschans erwägen. Iran reagierte auf die Propagandaschlacht seinerseits mit der unilateralen Abrogation der Artikel V und VI des Freundschaftsvertrages von 1921, jedes Interventionsrecht der UdSSR damit bestreitend. ${ }^{317}$ Trotz Vermittlung des UNOGeneralsekretärs ließen die sowjetische Propagandaattacken auch in den Folgemonaten nicht nach - Moskau insistierte auf eine unilaterale Zusicherung Teherans, keine militärischen Stützpunkte fremder Staaten zu akzeptieren, wohingegen Mohammad Rezā maximal dazu

\footnotetext{
${ }^{314} \mathrm{Vgl}$. Agreement of Co-Operation, Signed at Ankara, 5.3.59, U.N. Secretariat, Treaty Series 327, No. 4725, S. 278-83; auch in: ALEXANDER/NANES, United States and Iran, S. 306f.; die USA schlossen ähnliche Abkommen mit der Türkei und Pakistan ab.

${ }^{315}$ Im Planungsstab bevorzugte man nun im Grundsatz den bilateralen Pfad der Rückversicherung. Vgl. Matthews Memo, 27.7.59, ,Near and Middle East 1959-61 ', Lot 67 D 548, S/P Records: 1957-1961, Box 154, RG 59, NACP; vgl. auch die Kontextualisierung in LENCZOWSKI, Support for Independence.

${ }^{316}$ Während seines Staatsbesuchs in Großbritannien behauptete der Schah, die USA hätten ein neues ,reasonable document" vorgelegt und darüber hinaus ,[...] promised to consider Iran's military needs more favourably."; Record of a Meeting, 10.5.59; die Briten registrierten die Zusagen der Bilaterals, konstatierten aber zugleich: „There are many qualifying clauses and the American undertaking is by no means an automatic guarantee."; PM/59/51 Note for Macmillan, 7.5.59, PREM 11/3397, TNA:PRO; die USA hatten am Ende stark auf einen Abbruch der Verhandlungen mit den Sowjets gedrängt und im Gegenzug begrenzte Neuformulierungen und eine leichte Erhöhung der Budgethilfe zugesagt. Vgl. State \#2023, 7.2.59, 788.5/2-759, CF 55-59.

${ }^{317} \mathrm{Vgl}$. Botschaft Teheran an AA, 7.3.59, B 12/1006, PAAA; Chruschtschow attackierte den Schah persönlich in öffentlichen Ansprachen. Moskau wies zudem die einseitige Aufkündigung der Artikel von 1921 zurück. Vgl. Alvand, Flirting with Neutrality, S. 435ff.; RAmAZANI, Iran's Foreign Policy, S. 282-5, 299-302; CIA, Central Intelligence Bulletin, 30.4.59, CIA-FOIA; Herter Memo, 23.2.59, FRUS 58-60, XII, S. 639-42; Hart Memo, 25.2.59, ,Iran Press and Public Opinion 1959', Lot 61 D 407, Suppl. Subject Files Relating to Iran, 1956-59, OiCIA/GTI, Box 21; zum Exilsender vgl. SCHIRAZI, Modernität, S. 41f.; die DDR-Staatsführung stimmte bald der Ansiedlung des Senders in Ostdeutschland zu. Siehe Rādmaneš an ZK der SED, 9.5.57, SAPMO-Barch, DY 30/IV $2 / 20 / 361$.
} 
bereit war, einen Verzicht auf die Stationierung von Raketen mit längerer Reichweite auszusprechen. Die sowjetisch-iranischen Beziehungen blieben bis Anfang der 1960er Jahre gespannt, was zur inneren Destabilisierung der Lage in Iran erheblich beitrug. ${ }^{318}$

Positiv aus Sicht Mohammad Rezās war dagegen, dass die heftigen sowjetischen Attacken gegen seine Herrschaft und ihn persönlich einige der zu erwartenden negativen Konsequenzen abschwächten, was die Reaktion der Eisenhower-Administration auf seine zweifelhaften Avancen gegenüber Moskau anging. ${ }^{319}$ Trotz aller Verärgerung sah man in Washington nun die Notwendigkeit, das Schah-Regime gegen die sowjetischen Angriffe zu verteidigen. Zur Aufmunterung gewährte die Administration im Herbst 1959 zudem eine moderate Aufstockung der Hilfszusagen. ${ }^{320}$ Symbolisch bedeutend aus Sicht der Iraner war im Dezember 1959 auch der Kurzbesuch Präsident Eisenhowers in Teheran im Zuge seiner „Good Will“-Tour, die ein fortgesetztes Interesse der USA signalisierte. Allerdings zerstörte Mohammad Rezā in gewohnter Manier jeden positiven Effekt des Besuchs, indem er eine unverbindliche Unterhaltung mit dem Präsidenten über die militärischen Kapazitäten Irans und die angebliche Sowjetisierung Iraks und Afghanistans in eine vollkommen fiktive Zustimmung Eisenhowers zu einem „crash program“ für die iranischen Streitkräfte mit dem Ziel einer Umwandlung in eine hoch moderne und mobile Armee mit modernsten Waffensystemen umdichtete. ${ }^{321} \mathrm{Im}$ Januar 1960 ging eine exorbitante Wunschliste des Schahs ein: Iran benötige sechs zusätzliche Militärflughäfen, zwei Radar-Frühwarnstationen, 150 moderne Century Class-Jagdbomber, dazu 36 taktische Bomber, drei Staffeln Transportflugzeuge, zwei Batterien Nike AjaxLuftabwehrraketen, und moderne M-48-Panzer. Er schlug die Umwandlung der iranischen Armee in zehn hochmobile „Battle Groups“ vor, ausgestattet mit atomwaffenfähigen Boden-

\footnotetext{
318 Vgl. Jones Memo, 23.7.59; Herter Memo, 16.9.59; ed. notes, FRUS 58-60, XII, S. 645ff., 652f., 669f., 690f.; Draft Report, o.D. [1959], ,USSR-Iran Relations (Jan.-March 1959)`, NEA, GTI: RIAD, 1958-63, Box 1; INR Study No. 8145, 2.11.59; Matthews Memo, 21.1.59, ohne Folder, Lot 67 D 548, S/P Records: 1957-1961, Box 141, RG 59, NACP; vgl. SiCKER, Bear and Lion, S. 89-92; ANDREW/MitROKHIN, The Mitrokhin Archive II, S. $171 \mathrm{f}$.

${ }^{319}$ Interessant und im Kern über die Leitlinien von NSC 5821/1 hinausgehend war die Erwähnung einer denkbaren Reaktion auf Mohammad Rezās Verhandlungen mit Moskau seitens Botschafter Wailes: „Consider campaign of political and subversive action to bring local pressure on Shah to change his mind or reduce him to figurehead [...].“; Telegram \#1425, 30.1.59, FRUS 58-60, XII, S. 630; Wailes zufolge würde eine solche Kampagne zu lange dauern und er verwarf daher die Option, doch illustriert allein die Erwähnung die Wahrnehmung der SchahHerrschaft Anfang 1959 und auch die Selbsteinschätzung des amerikanischen Einflusses in Iran.

${ }^{320}$ Vgl. ebd., S. $654 n 1$; unter anderem sagte man eine beschleunigte Lieferung der ersten F-86-Staffeln zu. Vgl. Herter Memo, 25.8.59; Jones Memo, 22.9.59, ebd., S. 649ff., 654f.; Note ,Aid Projects for Iran', 21.9.59, 788.5 MSP/9-2259, CF 55-59; Jones Memo, 3.10.59, ,Memos thru S/S for Clearance‘, Lot 61 D 407, Suppl. Subject Files Relating to Iran, 1956-59, OiCIA/GTI, Box 21; TelCon, 11.2.59, ,Memoranda of Tel.Conv. Jan 4, 1959 to May 8, 1959 (2)‘, JFDP, Telephone Conversations Ser., Box 9, DDEL.

${ }^{321}$ Eisenhower hielt sich nur vier Stunden in Iran auf. Zur Kurzvisite vgl. GoodE, United States and Iran, S. 165; BARRETT, Greater Middle East, S. 158; Mohammad Rezā informierte ARMISH-MAAG Chief Linquist über den angeblich detaillierten Austausch bezüglich der zukünftigen Streitmacht, angeblich ,atomic weapons, long-range missiles, effective anti-aircraft missiles, additional air bases, and improved aircraft" beinhaltend. Vgl. MemCon, 14.12.59; Herter Memo, 31.12.59; ed. note, FRUS 58-60, XII, S. 658f., 660f., 661f.; vgl. ebd., S.659 n2; CHARMIS-MAAG \#ARAA 86398, 19.12.59; Iranian Note ,Sovietization of Iraqi and Afghan Armies', encl. to Goodpaster Memo, 24.12.59, ,Iran (2) [September-December 1959]', SS, International Ser., Box 8, DDEL; Mohammad Rezā hatte Eisenhower diese Einschätzung über die rasant ansteigenden militärischen Kapazitäten seiner Nachbarn während seines Besuchs übergeben. Während seines London-Besuchs wenige Monate zuvor hatte der Schah weitaus wahrheitsgetreuer eingestanden: ,In spite of the communist foothold in Iraq, the Shah did not think that there was a danger to Persia from that quarter for the time being, since at the moment Iran was more of a danger to Iraq than the other way round."; Record of Meeting, 10.5.59, PREM 11/3397, TNA:PRO.
} 
Boden-Raketen, und erweitert um eine Fallschirmjäger-Brigade. Das Aufrüstungsprogramm war nicht nur wahnwitzig, was die Materialwünsche anging, sondern auch bezüglich des Preises - das DOD schätzte die Kosten eines solchen Programms auf exorbitante \$600 Millionen: ,[...] his request for military assistance [...] is not only excessive but is also beyond his capacity to use effectively.“322 Zur Enttäuschung des iranischen Monarchen ging Eisenhower in seiner Antwort nicht auf die detaillierten Wünsche ein. ${ }^{323}$

Die nicht enden wollenden Forderungen des Schahs nach beschleunigter Aufrüstung und hochmodernen Waffensystemen, weit jenseits dessen, was seine Streitkräfte realistisch zu absorbieren in der Lage waren, blieb eine Konstante in den bilateralen Beziehungen bis zum Ende der Eisenhower-Präsidentschaft. Intern war man sich nach Überprüfung der bestehenden Planungen bez. Militärhilfe an verbündete Staaten und angesichts der Haltung des USKongresses bewusst, dass eine Aufstockung des iranischen Programms undenkbar war. Am Ende von Eisenhowers Amtszeit war unmissverständlich klar geworden, dass Washington eine vollständige Umsetzung des Plan Counterbalance gemäß der Zusagen im Anschluss an die irakische Revolution nicht weiter beabsichtigte. Ein NEA-Offizieller stellte fest: ,[...] I do not believe that Iran's present and future depend upon rapid implementation of any military program. The reverse is probably true.“324 Aus Mangel an vorhandenen finanziellen Mitteln war man bereits 1959 weit hinter dem Zeitplan geblieben, sowohl was die Aufstockung der Armee wie auch den Aufbau militärischer Infrastruktur angingen. Den Empfehlungen des mit der Untersuchung des gesamten Militärhilfe-Programms beauftragten Draper Committee folgend, erstellte das Country Team an der Botschaft einen Fünfjahresplan für Iran, der eine schrittweise Reduzierung der US-Leistungen bei gleichzeitigem Anstieg der iranischen Verteidigungsausgaben um fast $70 \%$ in diesem Zeitraum, finanziert durch die Mehreinnahmen aus der Ölförderung, vorsah. Trotz des erheblichen Anstiegs der Ausgaben wären die Streitkräfte in Umfang und Ausrüstung weit hinter den Erwartungen des Schahs zurückgeblieben.

\footnotetext{
${ }^{322}$ Irwin Memo, 20.2.60, FRUS 58-60, XII, S. 666; ebd., $n 1$; Shah Letter, 12.1.60, ,Iran, 1960 (3) ${ }^{\star}$, AWF, International Ser., Box 32, DDEL; der Schah hatte die F-100 Super Sabre bzw. die gerade erst in Dienst gestellten F-105 Thunderchief-Jagdbomber und die B-57 Canberra-Bomber im Blick und er wünschte die hochmodernen Fairchild C-123 Provider-oder de Havilland Caribou-Transportflugzeuge. Für eventuelle atomare Einsätze sah er Honest John- bzw. Corporal-Kurzstreckenraketen als geeignet an. Die Marine wollte er unter anderem mit 8 Minenräumbooten und 4 Korvetten aufrüsten. Zugleich verlangte er eine Ausweitung der Trainingsmissionen, obgleich bereits 900 amerikanische Militärberater im Land waren.

${ }^{323}$ Eisenhower warnte vor Übertreibungen der irakischen und afghanischen Bedrohung und ermahnte Mohammad Rezā, die Einführung von „complicated and advanced weapons“ könne nur langsam und graduell erfolgen. Vgl. Eisenhower Letter, 12.3.60, ,Iran, 1960 (3)`, AWF, International Ser., Box 32, DDEL; vgl. FRUS 58-60, XII, S. $674 n 3$; Jones Memo, 18.2.60, deklassifiziert 2011 infolge eines FOIA-Antrags [NW 21608] des Verfassers.

${ }^{324}$ Mouser Memo, 25.5.59, ,U.S. Military Assistance to Iran 1959', Lot 61 D 407, Suppl. Subject Files Relating to Iran, 1956-59, OiCIA/GTI, Box 21; offenbar hatten der Schah und ARMISH-MAAG bereits eine Aufstockung der Sollstärke auf 230.000 Mann in die Wege geleitet. Vgl. Lincoln/Perkins Memo, 5.5.59, , Iran Country Budget"; ebd.; im State Department mehrte sich die Kritik an den Plänen. Man müsse sich fragen ob Counterbalance, ,does not envisage a more rapid build-up than was necessary to satisfy the Shah."; Rountree Memo, 16.6.59, 788.5 MSP/6-1659; Bell Memo, 8.6.59, 788.5 MSP/6-81959; McClelland Memo, 2.9.59, 788.5 MSP/9-359, CF 55-59; vgl. Country Team Meeting Minutes, 4.3.59, ,Iran Country Team Meetings $195920^{\circ}$, Lot 61 D 407, Suppl. Subject Files Relating to Iran, 1956-59, OiCIA/GTI, Box 20; Details zu Counterbalance in Bowling Memo, 21.11.60, FRUS 58-60, XII, S. 707.
} 
Aus amerikanischer Sicht war die (konventionelle) Verteidigung Irans keine Priorität mehr bzw. galt der Nahe Osten nicht mehr als primärer Austragungsort von Kampfhandlungen im Falle eines militärischen Konflikts mit der UdSSR. Mohammad Rezās Behauptungen einer Gefahr seitens Iraks und Afghanistans wurden in Washington als offenkundig vorgeschoben verworfen. ${ }^{325}$ Selbst nach zehn Jahren Militärhilfe und der Lieferung von Ausrüstung für fast \$400 Millionen sah man die Aufgabe der iranischen Streitkräfte zuvorderst in der Bewahrung innerer Sicherheit und, maximal, in der Behinderung und kurzzeitigen Verzögerung eines eventuellen sowjetischen Vorstoßes. Der Schah drohte weiterhin, wenig glaubwürdig, mit einem Rückzug aus der pro-westlichen regionalen Verteidigungsorganisation, die im Anschluss an den formellen Austritt Iraks im März 1959 im August in Central Treaty Organization (CENTO) umgetauft wurde. Wie seine Vorgängerversion, litt auch CENTO unter dem mangelnden Engagement seiner Mitglieder und unter der amerikanischen Weigerung dem Bündnis beizutreten. Eine amerikanische Studie der militärischen Bedeutung der CENTO vom Oktober 1960 kam zu dem Schluss, dass das Bündnis weder über ein Oberkommando und genehmigte Einsatzpläne, noch über die notwendige Koordination mit NATO oder SEATO verfügte. CENTO blieb in vielerlei Hinsicht eine Chimäre. ${ }^{326}$ Im Anschluss an zwei Kolumnen des bekannten amerikanischen Journalisten Walter Lippman im Dezember 1959, der die gegenwärtige Iranpolitik kritisierte und die Administration ermutigte, sich mit den strategischen

${ }^{325}$ Im März 1960 kündigte der Schah sogar eine beschleunigte Erhöhung der Sollstärke auf 240.000 Mann bis Ende des Jahres an. Vgl. ebd., S. 674 1; vgl. Herter Memo, 19.9.60, ebd., S. 700f.; Shah Letter, 30.3.60; Eisenhower Letter, 20.9.60, ,Iran, 1960 (1)', AWF, International Ser., Box 32, DDEL; obwohl der provisorische Fünfjahresplan weit hinter den Erwartungen des Schahs und den Militärplanungen des Bagdadpaktes zurück blieb, sah Bowling, inzwischen Iranian Affairs innerhalb GTIs leitend, auch ein solches abgespecktes Programm als wirtschaftlich nicht tragbar an: „All of this means that Iranian economic development will be slowed to a walk with the enormous growth of local military expenditures. The political results would be obvious.“; die Idee eines koordinierten Angriffs der Nachbarn auf Iran sei ,,[...] so plausibly flimsy and ridiculous that it is not necessary to list the arguments against the idea. It was never more than a rationalization to cover a basic desire for bigger and more modern armed forces per se."; Bowling Memo, 21.11.60, FRUS 58-60, XII, S. 709; ähnlich die britische Einschätzung: ,It seems unlikely that Afghanistan, even with Soviet backing, at present poses any serious threat, either political or military, to Iran."; J.I.C.(59) 24 (Final), 12.3.59, CAB 158/35, TNA:PRO; selbst eine 400.000Mann-Armee mit modernen Waffen könne die Sowjets im Kriegsfall nur einige Tage aufhalten. Nur mit ca. $\$ 500$ Millionen pro Jahr könne man innerhalb von 5 bis 10 Jahren eine Streitmacht mit einer Kampfkraft vergleichbar zu den türkischen Streitkräften generieren, was offenkundig weder finanzierbar noch politisch durchsetzbar sei, so Bowling. Vgl. auch Marcy Memo, 19.7.60; O/NESA Comments, o.D., ,3-A/1 Military Assistance, Iran 1960‘, NEA, GTI: RIAD, 1958-63, Box 2 .

${ }^{326}$ Vgl. JCS History Iran, S. 60; FAIRCHILD/PoOLE, History of the JCS: VII, S. 172-5; COHEN, Defending the Northern Tier, S. 212ff.; Meeting Minutes, ,State-JCS Meeting, March 11, 1960', Lot 70 D 328, State-JCS Meetings: 1959-63, Box 1; die JCS forderten im Oktober 1960 ein weiteres Mal eine Aufwertung der CENTO und einen US-Beitritt. Vgl. Smith Memo, 25.4.60; Jones Memo, 23.11.60, ,Near and Middle East 1959-61 ', Lot 67 D 548, S/P Records: 1957-1961, Box 154, RG 59; eine Demonstration der Fähigkeiten des amerikanischen Tactical Air Command in den CENTO-Staaten, Operation QUICK SPAN vom Februar 1960, im Iran abgehalten vor den Augen der gesamten politischen und militärischen Führung und etwa 50.000 Zuschauern, wurde aber offenbar als erfolgreiche Bekräftigung der amerikanischen Sicherheitsgarantien wahrgenommen. Vgl. Knight Memo, 27.4.60, ,CCS 9070/3510 Baghdad Pact, CENTO (30 Jan 1960)`, JCS Central Decimal File 1960, Box 68, RG 218, NACP; Mohammad Rezā präferierte zeitweilig eine regionale Alternative zur CENTO. Im September 1958 verkündete er Gespräche mit Afghanistan, Pakistan und der Türkei über die Schaffung einer, ,Arischen Union“ als Gegenmodell zu den panarabistischen Einigungsbemühungen der VAR. Die Eisenhower-Administration sah ,many practical difficulties to integrating these countries on any but a «paper» basis"; NSC 5821/1, S. 609; siehe auch Rountree Memo, 9.6.58, ebd., S. 561; PM/58/36 Memo, 28.4.58, PREM 11/2409, TNA:PRO; vgl. RAMAZANI, Iran's Foreign Policy, S. 284. 
Vorzügen einer iranischen Neutralität ernsthaft zu befassen, wuchs die Angst in Teheran vor einem strategischen Rückzug der Schutzmacht. ${ }^{327}$

Mohammad Rezās unverminderte Fokussierung auf die militärische Aufrüstung und seine demonstrierte Bereitschaft, die USA mittels Verhandlungen mit den Sowjets unter Druck zu setzen wenn nicht gar zu erpressen, trugen zu einer weiteren Abwertung seines Ansehens in Washington bei. Der Flirt mit Moskau und der Abschluss der Bilaterals hatte temporär die politische Aufmerksamkeit von den in NSC 5821/1 vom November 1958 festgelegten neuen Politikzielen der USA abgelenkt. Im April 1959 erkundigte sich das State Department ungeduldig über die Absichten Mohammad Rezās bezüglich des von ihm zugesagten „meaningful program of internal reform“."328 Aus Sicht amerikanischer Entwicklungsökonomen war gerade die Beseitigung von Modernisierungshemmnissen in den ländlichen Regionen ein wichtiger Schwerpunkt ihrer Tätigkeit in den 1950ern gewesen. Der Reformdruck der Eisenhower-Administration nahm nun so stark zu, dass der Schah einwilligte, eine wesentlich von amerikanischen Experten verfasste Landreformgesetzgebung in den Mağles einzubringen - verabschiedet im März und Mai 1960 von beiden Kammern. Die verabschiedete Version enthielt nun aber erhebliche Abweichungen vom ursprünglichen Entwurf, wodurch die Reform entscheidend verwässert und letztlich nie umgesetzt wurde. ${ }^{329}$ Im Laufe des Jahres 1959 vermehrten sich zudem Anzeichen für eine schwerwiegende wirtschaftliche Krise, ausgelöst durch inflationäre Entwicklungen infolge der stetig anwachsenden staatlichen Ausgaben in Kombination mit den stark steigenden kreditfinanzierten Investitionen im Privatsektor. Die geplanten Ausgabensteigerungen im Rahmen des Zweiten Siebenjahresplans (1955-62) drohten in eine Zahlungsbilanzkrise des iranischen Staates zu münden. Die iranische Regierung musste daher 1960 vorübergehende Finanzhilfe vom Internationalen Währungsfonds beantragen und in diesem Kontext ein Stabilisierungsprogramm aushandeln. ${ }^{330}$

Trotz sichtbarer Ermüdungserscheinungen auf amerikanischer Seite unterstützte die Administration diese Bemühungen ein weiteres Mal. Für das Finanzjahr 1961 sagten die USA Wirtschaftshilfe in Form von Darlehen über etwa \$50 Millionen - der Währungsfonds gewährte Kredite in vergleichbarer Höhe - und, trotz der wiederholt geäußerten Entschlossenheit diese

\footnotetext{
${ }^{327}$ Vgl. Nussbaum Memo, 15.12.59, ,Iran, Press and Public Opinion 1959‘, Box 20; Jones Memo, 31.12.59, ,Memos Thru S/S for Clearance - 1959“, Lot 61 D 407, Suppl. Subject Files Relating to Iran, 1956-59, OiCIA/GTI, Box 21; vgl. Eintrag vom 15.1.59, in: LILIENTHAL, Journals: $I V$, S. $301 \mathrm{f}$.

${ }^{328}$ State \#2748, 30.4.59, 611.88/4-3059, CF 55-59; ein Progress Report vom Dezember 1959 konstatierte kleine Anzeichen für Reformbereitschaft, die aber, ,slow in coming, and even slower in its impact upon the general populace" sei. Progress Report on NSC 5821/1, 9.12.59, ,430th Meeting', Official Meeting Minutes, Box 24, RG 273, NACP.

${ }^{329}$ Siehe FRUS 58-60, XII, S. $671 n 1$; bereits im Oktober 1958 hatte das Kabinett in einem kleinen ersten Schritt die quasi-feudalen Abgaben beseitigt, um die Lasten der Landbevölkerung zu verringern. Vgl. Tehran \#297, 27.10.58, 788.00/10-2758, CF 55-59; vgl. NAJMABADI, Land Reform, S. 74f.; NEMCHENOK, Rural Development. ${ }^{330}$ Vgl. Progress Report on NSC 5821/1, 9.12.59, ,430th Meeting', Official Meeting Minutes, Box 24, RG 273; Miklos Memo, 23.9.60, ,Economic Stabilization Mission 1960`, NEA, GTI: RIAD, 1958-63, Box 2; vgl. KATOUZIAN, Political Economy of Modern Iran, S. 202-7; ABRAHAMIAN, Two Revolutions, S. $421 \mathrm{f}$.
} 
Art von Unterstützung einzustellen, auch einen weiteren Budgetzuschuss über \$22 Millionen zu. Doch waren es nicht allein die Effekte der Pfadabhängigkeit von der bisher verfolgten Politik, welche die erneuten Hilfszusagen determinierten. Denn nur über die Gewährung von Wirtschaftshilfe erlangte Washington effektiven Zugang zu den wichtigsten Personengruppen in Verantwortung für die iranische Wirtschafts- und Finanzpolitik und darüber hinaus die Möglichkeit, auf deren Entscheidungen und Planungen Einfluss zu nehmen. Zudem hatte die intensive und langjährige Involvierung in die Entwicklungsbemühungen enge professionelle wie persönliche Beziehungen zwischen amerikanischen und iranischen Beratern, Offiziellen und Entwicklungsexperten produziert. Mohammad Rezā nutzte dies geschickt, indem er im Kontext der Wirtschaftskrise vom Sommer 1960 eine vorwiegend mit Personen aus dem Umfeld des pro-amerikanischen Netzwerks besetzte Delegation nach Washington entsandte. Im OCB betonte man, dass ,the fact of the mission and its composition“ Ausdruck eines ,new look", einer nun realistischen und auf (westlicher) Expertise basierten Entwicklungsvision, innerhalb der iranischen Regierungsbürokratie seien. ${ }^{331}$ Unterstützt wurden die iranischen Wünsche nach Fortsetzung des Hilfsprogramms von den amerikanischen ,Men on the Spot": Ebtehāğ, der Direktor der Planorganisation, hatte mit Unterstützung der Ford Foundation amerikanische Consultants angeworben - im Mai 1958 war die Harvard Advisory Group eingetroffen und half fortan beim Aufbau eines Economic Bureau innerhalb der Planorganisation. Kenneth Hansen, der Leiter der Harvard-Gruppe, unterstützte aktiv die iranischen Wünsche nach weiteren Hilfszusagen. ${ }^{332}$

Ebtehāăg selbst, die dominierende Figur innerhalb der iranischen Wirtschafts- und Entwicklungsplanung und jahrelang de facto Leiter einer stark amerikanisch beeinflussten Schattenregierung in ökonomischen Belangen, war im Februar 1959 als Direktor der Planorganisation zurückgetreten, nachdem Mohammad Rezā der vielen Konflikte über

${ }^{331}$ OCB Working Group on Iran MemCon, 23.6.60, ,OCB 091 Iran Folder 5‘, Records of the OCB, Box 3, RG 273, NACP; vgl. FRUS 58-60, XII, S. 712 n1; State \#24, 1.7.60; Jones Memo, 20.9.60, ebd., S. 679f., 701-4; MemCon, 23.6.60, 788.5-MSP/6-2360, CF 60-63; auf Anraten der Botschaft hatte die Eisenhower-Administration ursprünglich eine spürbare Reduktion der US-Hilfsleistungen beschlossen, mit dem Ziel, die Iraner zugunsten größerer ökonomischer Vernunft und stärkerer Ausgabendisziplin zu erziehen. Vgl. Jones Memo, 8.12.59, OCB Operations Plan, 30.4.58, ,Iran 1959-1960‘, Lot 62 D 430, Regional and Country Operations Files, 1953-1961, Box 21.

${ }^{332}$ Ebtehāğ hatte den Harvard-Ökonomen Edward S. Mason um Unterstützung gebeten, um geeignetes Personal zu finden. Die Harvard Advisory Group wurde letztlich von Kenneth Hansen geleitet, später Assistant Director of the Bureau of the Budget unter der Kennedy-Administration und einer der wichtigsten amerikanischen reformistischen Interventionisten. Vgl. Bostock/JONES, Planning and Power, S. 122f.; zu Hansen vgl. GoODE, United States and Iran, S. 171; BREW, Second Seven Year Plan; Hintergründe bei Xodādād Farmānfarmā ijān $\mathrm{OHI}, 10.11 .82$, IOHP; trotz früher Anzeichen für Zahlungsbilanzschwierigkeiten drängte Hansen im Sommer und Herbst 1959 auf weitere Darlehen im Anschluss an eine erneute IBRD-Zusage über \$72 Millionen. Die IBRD gewährte zusätzliche \$42 Millionen im Februar 1960. Vgl. MemCon, 24.9.59, , Iran, Development Loan Fund 1959', Lot 61 D 407, Suppl. Subject Files Relating to Iran, 1956-59, OiCIA/GTI, Box 20; bereits im Zusammenhang mit dem IBRD-Darlehen hatte die iranische Regierung formal weitreichende Zusagen bez. Ausgabenbeschränkungen und budgetärer Reformschritte zugesagt. Siehe Jones Memo, 1.6.59, ,Iran IBRD 1959`, ebd., Box 21; der Botschaft zufolge war die Präsenz der Harvard Group innerhalb der Planorganisation ,of considerable help to the mission in its relations to the Iranian economic development program", doch gestand man zugleich eine gelegentliche ,[...] over-sensitivity on the part of Economic Bureau personnel regarding their position within an Iranian institution, which they wish to keep from being compromised by over-identification with U.S. elements" ein; Tehran \#G-39, 3.10.59, 788.5MSP/10-359, CF 55-59; ein ehemaliger US-Offizieller beschrieb den Mittelgewährungsprozess für die US-Entwicklungshilfe, wobei man mittels eines Master Joint Fund de facto eine Art Schattenregierung geführt habe. Siehe Maurice Williams OHI, 15.5.96, FAOH. 
Zielsetzungen und Finanzierung der Entwicklungspläne überdrüssig geworden war und schließlich einer Übertragung der Wirtschaftssteuerung auf das Amt des Ministerpräsidenten zugestimmt hatte. ${ }^{333}$ Angesichts der drohenden Entmachtung der Planorganisation war man nun auf US-Seite umso mehr auf Fortsetzung der bisherigen Zusammenarbeit bedacht. Eine GTI-Analyse der wirtschaftlichen Gesamtsituation betonte die Schlüsselrolle Mohammad Rezās, was die weitere Entwicklungsorientierung Irans anging. Er allein garantiere, dass auch weiterhin wenigstens $55 \%$ der Öleinnahmen für Entwicklungsziele verwendet würden und er allein garantiere die Sonderstellung der pro-amerikanischen Planer. Daher solle man die bisherige Politik fortsetzen und weiterhin ,_...] carefully support some of the promising moderates of the left and right who are now moving slowly but surely ahead on stabilization and economic development programs." Zugleich aber müsse dies mit Vorsicht vonstattengehen, damit Mohammad Rezā diesen Personenkreis nicht als Bedrohung wahrnehme und ,[...] promptly cut them off; a step he could easily take at this juncture.“334

Präsident Eisenhower selbst kommentierte im März 1960 im NSC das Dilemma der amerikanischen Iranpolitik. Obgleich der Schah nun endlich ein Landreform-Programm angekündigt habe und somit den Wünschen Washingtons entspreche, sorge man sich weiterhin um die Stabilität in dem Land. „Our policies were apparently not effective enough in properly orienting the Iranian people“, mutmaßte Eisenhower über die von ihm selbst abgesegnete Iranpolitik der vergangenen acht Jahre. ${ }^{335}$ Im Juni ging Eisenhower noch weiter mit seiner Kritik an der bisherigen politischen Linie der von ihm angeführten Administration. Die seiner Meinung nach weltweit zu konstatierende Abnahme an Stabilität führte er auf die Prävalenz autoritärer Herrschaftssysteme zurück. Man müsse die Völker auf „,free self-government“

\footnotetext{
${ }^{333}$ Tatsächlich war man auf amerikanischer Seite trotz Respekt für Ebtehāğ und Anerkennung seiner Leistungen erleichtert über seinen Abgang aufgrund des äußerst schwierigen persönlichen Umgangs mit ihm und seiner fast vollständigen Isolierung innerhalb des Teheraner Establishments. Bereits 1955 notierte man im OCB, Ebtehāğs „uncompromising attitude“" sei zumindest teilweise dafür verantwortlich, dass, ,it has not been possible for the U.S. to exercise such influence on the choice and magnitude of projects selected for immediate financing [...]. “; OCB Draft Report (NSC 5504), 8.6.55, ,OCB 091. Iran (File \#2) (8)‘, WHO, NSCS, OCB Central File Ser., Box 43, DDEL; 1956 konstatierte man in GTI, Ebtehā s̆ sei ,,[...] widely hated and must be counted a political liability."; Kitchen Memo, 17.5.56, FRUS 55-57, XII, S. 818; Anfang 1957 berichtete die Botschaft, der Hass auf Ebtehāğ in Mağles und Regierungskreisen „has become almost pathological“; Tehran \#1130, 23.1.57, ebd., S. 880; Sonderbotschafter Richards kritisierte nach seiner Rückkehr aus Iran Ebtehāğs Führung im Kongress so scharf, dass sich GTI zu einer Replik genötigt sah. GTI Comments, 13.6.57, encl. to May Memo, 17.6.57, ,Iran Richards Mission 1957‘, Lot 60 D 533, Subject Files Relating to Iran, 1951-1958, OiCIA/GTI, Box 10; der Schah nahm Ebtehā $\breve{g}$ offensichtlich auch die Anstellung ehemaliger Parteigänger der Nationalen Front übel - ,those people who broke my statues" - seine Replik sei nicht bekannt, ,[...] but it was probably on the lines that they were the only people he could find who were both efficient and honest. There may, unfortunately, be truth in this", so die britische Gesandtschaft. Chancery to FO, EP1015/4, 19.1.57, FO 371/127074, TNA:PRO; vgl. auch den Eintrag vom 1.12.56, in: LILIENTHAL, Journals: $I V$, S. 131ff.

${ }^{334}$ Miklos Memo, 28.12.60, ,Economic Stabilization Mission 1960‘, NEA, GTI: RIAD, 1958-63, Box 2; so lobte Eisenhower in einem Brief an Mohammad Rezā ausdrücklich die ,,ability“ und „determination“" der fast ausschließlich aus pro-amerikanischen Netzwerkern bestehenden Stabilisierungsdelegation, die im Sommer 1960 nach Washington entsandt worden war. Eisenhower Letter, 20.9.60, S. 204, Anm. 325; man war sich bewusst, dass die notwendigen Kürzungen des Planungsbudgets , , [... ] might have the effect of substantially curtailing or even eliminating the Clapp-Lilienthal project in Khuzistan."; die Botschaft in Teheran habe ohnehin erhebliche Zweifel bez. der Sinnhaftigkeit der Tätigkeit der Development and Resources Corporation. Siehe Jones Memo, 1.6.59, S. 206, Anm. 332; der Mağles beendete 1960 die Autonomie des Unternehmens in Xuzestān. Vgl. FISHER, Lilienthal, S. 445; Einträge vom 3.3.59, 25.5.59, in: LILIENTHAL, Journals: $I V$, S. 314-7, 346f.; Einträge vom 10.8.59, 25.11.60, in: LiLIENTHAL, Journals: $V$, S. 2ff., 137-40.

${ }^{335}$ Zit. aus dem 436th NSC-Meeting am 10.3.60 in ed. note, FRUS 58-60, XII, S. 670.
} 
vorbereiten und die USA demnach ,indigenous individuals who are true liberals“ unterstützen. Bezüglich Iran kommentierte Eisenhower, es befände sich in einer beinahe ähnlich schwierigen Lage wie in der Mosaddeg̈-Ära und er kam zu der erstaunlichen Schlussfolgerung: „The situation there might be improved if the liberals could succeed in deposing the Shah and taking over the government. “336 Diese Äußerungen deuteten zumindest im Ansatz eine Abkehr von den Leitlinien gemäß NSC 5821/1 vom November 1958 an, mit ihrer Fokussierung auf den Schah als „best hope“ für transformative Reformen in Iran. Hoffnungen auf eine konsequentere reformistische Intervention in Iran erfüllten sich aber nicht. Zwar entschloss man sich zur Formulierung einer neuen und letzten NSC-Studie über die eigene Iranpolitik, doch handelte es sich im Kern nur um ein begrenztes Aufdatieren der älteren Studie im Allgemeinen, als General Considerations titulierten Teil. Die Objectives und die Policy Guidance-Sektionen von NSC $5821 / 1$ dagegen wurden unverändert übernommen. Somit stellte NSC 6010, vom NSC am 30. Juni 1960 verabschiedet, eine Fortsetzung der bisherigen Politik dar, trotz aller durchaus registrierten grundsätzlichen Defizite der eigenen Strategie. ${ }^{337}$ Tatsächlich hatte das State Department in den Beratungen sogar auf eine Abschwächung bzw. Streichung der konkreten Reformschritte gedrängt, die noch in NSC 5821/1 angemahnt worden waren. Einigen Stimmen im State Department zufolge gebe es in Iran überhaupt kein Potential für eine ,popular“ oder „grass roots revolt“. Nur wenige Iraner besäßen ein echtes politisches Bewusstsein und die grundlegende Charaktereigenschaft der gebildeten Iraner könne am besten mit „opportunistic individualism“ beschrieben werden. Da die Hauptgefahr für die Schah-Herrschaft somit allein ein Staatsstreich vonseiten der Armee sei, könne die Schwerpunktsetzung auf innere Reformen getrost vernachlässigt werden, so diese Stimmen. ${ }^{338}$ Tatsächlich gelang es dem State Department, die reformistischen Formulierungen im Operations Plan for Iran vom Oktober 1960, also den Umsetzungsleitlinien für das Feld, entscheidend abzuschwächen. Eine wortwörtliche Umsetzung von NSC 6010, so versicherte man sich selbst im State Department, „[...] would go far beyond the dictates of common sense.“" ${ }^{339}$ Der Operations Plan stellte dann

336 449th NSC Meeting, 30.6.60, $449^{\text {th }}$ Meeting of NSC', AWF, NSC Ser., Box 12, DDEL; einige dieser Aussagen auch in der FRUS-Version der Minutes. Siehe FRUS 58-60, XII, S. $676 \mathrm{ff}$.

${ }^{337}$ Vgl. ebd., S. 672f. $n 6$; 449th NSC Meeting, 30.6.60, ebd., S. 676ff.; NSC 6010, ,U.S. Policy Toward Iran‘, encl. to Lay, Jr., NSC Council Report, 6.7.60, ebd., S. 680-8; Briefing Note for Planning Board, 21.3.60, ,[Iranian Political Situation...][1957-60]', WHO, OSANSA, NSC Ser., Briefing Notes Subs., Box 11; Halla Memo, 31.3.60; Buford Briefing Note, 6.6.60, ,Iran (1) ${ }^{*}$, Special Staff File Ser., Box 4, DDEL; das vollständige Dokument mit allen Anhängen in ,Iran', Lot 67 D 548, S/P Records: 1957-1961, Box 130, RG 59, NACP; Entstehung von NSC 6010 gänzlich missverstanden in LITTLE, American Orientalism, S. 218.

${ }^{338}$ Die vorgeschlagenen Reformen, ,tend to appeal to an American rather than an Iranian mentality. Consequently, there appear to be real dangers in U.S. promotion of and inevitable identification with specific reform measures in a country where political attitudes and reactions vary so significantly from our own."; Knight Memo, 6.6.60, ,OCB 091 Iran Folder 5', Records of the OCB, Box 3, RG 273, NACP; Knight hatte zuvor eine konkrete Ablehnung einer Landreform aus dem Memo streichen lassen. Letztlich holte sich das State Department bei dem Versuch der Abschwächung der NSC-Studie eine „,bloody nose“. Niemand „desired to make any real changes in NSC 5821/1.“; Knight Memo, 3.6.60; Rivinus, Jr. Memo, 8.6.60, ebd.; die Kritik bezog sich vor allem auf den reformistischen Paragraph 28 der bisherigen NSC-Studie. Siehe NSC 5821/1, S. 612.

${ }^{339}$ Rivinus Jr. Memo, 29.9.60, ,OCB 091 Iran Folder 6', Records of the OCB, Box 3, RG 273, NACP; hier wurde explizit auf den Einfluss des neuen Iran Desk Officer Bowling verwiesen. Sowohl in NSC 5821/1 und NSC 6010 war die Wendung ,to induce, and where appropriate, press the Shah“ bez. der Einleitung von Reformen enthalten. Das State Department versuchte, dies nun mit „should, whenever possible, attempt to encourage the Shah“ zu ersetzen, somit auf die Anwendung von Druck seitens der USA explizit verzichtend. Der OCB beugte sich nicht, stimmte aber einer Reihe von anderen Abschwächungen zu. Vgl. Memo of Meeting of OCB Working Group on Iran, 29.9.60, ebd.; zudem verschob man Contingency Planning im Falle einer entscheidenden Schwächung des 
auch unmissverständlich fest: „There is no alternative to the Shah which would not be retrograde in terms of U.S. interests under present circumstances. “"340

Nicht alle Offiziellen in der Administration teilten den grundsätzlichen Optimismus der Diplomaten mit Blick auf Iran. Gerade die CIA wurde nun zur Fürsprecherin eines konsequenteren Eingreifens in Iran, um die notwendigen Reformen in Iran zu erzwingen und eine ansonsten unausweichliche Revolution abzuwenden. Pikiert notierte man in Foggy Bottom, die CIA sei offenbar überzeugt, dass , ,[...] the Shah's days are numbered, and that even NSC 6010 is over-optimistic." ${ }^{341}$

Tatsächlich verwies ein NIE vom Februar 1960 auf den generellen Trend einer zunehmenden Destabilisierung der internen Lage in Iran aufgrund der wirtschaftlichen Krise. Gleichzeitig gestand die CIA eine gewisse Verbesserung der Position Mohammad Rezās ein, der „,considerable skill in keeping the opposition fragmented and on the defensive" gezeigt habe. ${ }^{342}$ Ähnlich wie das State Department zweifelte man in der CIA an der Möglichkeit eines Aufstands seitens der Bevölkerung, befürchtete aber weiterhin Unruhe in den Streitkräften. Insbesondere nach dem Staatsstreich in der als stabiler geltenden Türkei vom Mai 1960 stieg die Besorgnis. Man verfüge über Informationen über fortlaufende Coup-Planungen in der Armee, und ein Staatsstreich könne jederzeit und ohne Vorwarnzeit erfolgen, so die CIA. ${ }^{343}$ Angeblich erstellte SAWAK-Direktor Baxtijār bereits Notfallpläne für den Fall eines Kontrollverlustes oder Ablebens Mohammad Rezās, was nicht wenige als Tarnung für eigene Staatsstreichpläne interpretierten. Die Teheraner Botschaft hingegen wandte sich gegen den geheimdienstlichen

Schahs in ein geheimes Supplement zum Plan. Vgl. OCB, Operations Plan for Iran, 19.10.60; Supplement to Operations Plan for Iran, 19.10.60; ebd.; für einen direkten Beleg der Absicht des State Departments, über diesen Umweg NSC 6010 zu sabotieren, siehe Rivinus Jr. Memo, 29.7.60, ebd.

340 Operations Plan for Iran, S. 4, vorherig. Anm.

${ }^{341}$ Zit. aus Rivinus Jr. Memo, 29.9.60, Anm. 339; zu den Gegensätzen auch Jones Memo, 10.10.60, ,Iran 19591960“, Lot 62 D 430, Regional and Country Operations Files, 1953-1961, Box 21; allerdings wuchs zugleich die Bedeutung Irans für die amerikanische intelligence community infolge der Ansiedlung neuer Horchstationen im Norden Irans ab 1957, gerichtet u.a. auf Raketen- und Atomwaffentests der UdSSR. 1960 forderte das DOD den Aufbau einer weiteren Station $\left(\right.$, Red Wind $\left.{ }^{*}\right)$ innerhalb der US-Botschaft in Teheran. Ein Offizieller warnte, dass diese Station und eine offenbar bereits bestehende andere Station innerhalb des Botschaftsgeländes die ,diplomatic inviolability of the Embassy“ in Frage stellten. Vgl. Newman Memo, 3.11.60, 788.5631/11-360, CF 60-63; die ersten seismischen Stationen wurden offenbar bereits Ende 1952 in Iran aufgebaut. Vgl. BARRETT, Greater Middle East, S. 80; RicHELSON, Spying on the Bomb, S. 98, 115; bei Red Wind handelte es sich offenbar um akustische Stationen gerichtet auf das sowjetische Raumfahrt- und Raketenprogramm. Siehe ebd., S. 188.

${ }^{342}$ Vgl. CIA, NIE 34-60, 16.2.60, FRUS 58-60, XII, S. 665; ein Jahr zuvor hatte man die Chancen des Schahs, in den nächsten 1-2 Jahren in seiner Position zu verbleiben, nur als ,,somewhat better than even“ eingeschätzt. Siehe CIA, NIE 34-59, 3.3.59, ebd., S. 643; das britische Joint Intelligence Committee hatte im März 1959 eine „brüchige“ interne Lage in Iran konstatiert, sah aber das Schah-Regime allein durch einen Staatstreich, nicht durch einen Volksaufstand, gefährdet. Ein solcher sei in den kommenden zwölf Monaten aber unwahrscheinlich. Vgl. J.I.C. (59) 14 (Final), 5.3.59, CAB 158/35, TNA:PRO.

${ }^{343}$ Vgl. die Warnungen des Acting Director der CIA im NSC in 449th NSC Meeting, 30.6.60, ebd., S. 676; Tehran \#808, 14.6.60, 788.00/6-1460, CF 60-63; die britische Botschaft sah die Überlebenschancen des Schahs nun sehr skeptisch. Vgl. State \#3807, 9.6.60, deklassifiziert 2011 infolge eines FOIA-Antrags [NW 21263] des Verfassers. Die CIA beruhigte intern, das iranische Offizierskorps bestünde aus ,reluctant dragons“ und besäße nicht ,,the unity and sense of public responsibility that characterize the Turkish and Pakistani officer corps“. Allerdings seien im Falle von ,widespread public disturbances“ die Chancen für ein politisches Überleben des Schahs eher schlecht. vgl. CIA Memo for the Director, 3.6.60, CIA-FOIA; die türkische Armee hatte im Mai 1960 die demokratisch gewählte Regierung von Adnan Menderes gestürzt. 
Alarmismus - zwar könne ein Coup-Versuch nie ausgeschlossen werden, doch gäbe es ,,no real threat to the continuation of the Shah's regime on the immediate horizon". 344

Entgegen der Erwartungen der CIA wie auch des State Department sollten die nachfolgenden Entwicklungen zeigen, dass die Hauptgefahr für die Schah-Herrschaft in der nahen Zukunft tatsächlich von der breiten Bevölkerung und nicht in erster Linie von unzufriedenen Offizieren in den Streitkräften ausging. Ausgelöst wurde die innenpolitische Krise von einer Entscheidung Mohammad Rezās, die bereits auf den Juli 1960 verschobenen Wahlen zum 20. Mağles freier zu gestalten - im Juni verkündete der Schah vor beiden Häusern des Parlaments einen offenen und unbehinderten Wettbewerb zwischen den beiden „offiziellen Parteien“ Mardom und Mellijun. Anfangs wohl intendiert als Propagandacoup, verlor man alsbald die Kontrolle über die politische Öffnung und eine unerwartet breite politische Mobilisierung im politischen Feld Irans brach sich Bahn. Neben unerwartet heftigen politischen Debatten zwischen den beiden offiziellen Parteien beteiligten sich nun die Überbleibsel der Nationalisten Mosaddeġs und der NWI - sie schlossen sich jetzt peu à peu zur Zweiten Nationalen Front (ğebhe-je melli-je dowwom) zusammen - sowie eine konservative Opposition (,die Unabhängigen“), die sich locker um den diskreditierten ehemaligen Botschafter in Washington 'Ali Amini gruppierte. Zur allgemeinen Enttäuschung zeichnete sich in den Wahlen im Sommer eine klare Mehrheit für Eg̉bāls Mellijun ab, augenfällig gesteuert durch die üblichen Absprachen und Manipulationen, vor allem seitens des SAWAK. „Completely rigged“, konstatierte denn auch die US-Botschaft. ${ }^{345}$

\footnotetext{
${ }^{344}$ Tehran \#28, 20.7.60, FRUS 58-60, XII, S. 688; vgl. ed. note; 440th NSC Meeting, 7.4.60, ebd., S. 669f., 671ff.; Allen Dulles hatte dem NSC im Januar über ,serious plots against the Shah of Iran“ berichtet. Baxtijār werde als einer der möglichen Anführer eines Putsches genannt. Siehe 432nd NSC Meeting, 14.1.60, ,432nd Meeting of NSC', AWF, NSC Ser., Box 12, DDEL; die Botschaft berichtete von ,indications", Baxtijār erwäge sich an die Spitze der Regierung zu setzen und habe gewarnt, die gegenwärtige Politik des Schahs führe direkt zur Revolution. Siehe Tehran \#417, FRUS 58-60, XII, S. 694f.; basierend auf einem Interview mit Kim Roosevelt behauptete RUBIN, Good Intentions, S. 108f., Baxtijār habe ihn und Allen Dulles über seine Coupabsichten informiert. Beide hätten amerikanische Unterstützung verweigert und insgeheim den Schah über Baxtijārs Illoyalität informiert. Der Verblieb Baxtijārs im Amt bis 1961 spricht gegen diese Version. Pollack, Persian Puzzle, S. 79, wiederholt die Legende ebenfalls. Die Teheraner Botschaft berichtete, der SAWAK-Direktor sei bei ärmeren Schichten und bei den Stämmen durchaus populär, wenngleich verhasst bei den neuen Mittelschichten und unter den sogenannten „1.000 Familien" der reichen Oberschicht. Vgl. Rockwell Letter, 28.3.60, ,Official-Informal Letters from Tehran 1960", NEA, GTI: RIAD, 1958-63, Box 1; der Propagandasender Pejk-e Irān schürte bewusst Gerüchte über Coupabsichten Baxtijārs mit dem Ziel ,to eliminate potential pro-Western successors of the Shah", wie die CIA mutmaßte. CIA, Central Intelligence Bulletin, 9.3.60, CREST: \#RDP79*001-7; die Botschaft erstellte eine lange Liste möglicher Verschwörer, hielt aber dennoch an ihrer Überzeugung fest, dass ein Putschversuch eher unwahrscheinlich sei. Vgl. Tehran \#28, 20.7.60, deklassifiziert 2011 infolge eines FOIA-Antrags [NW 21263] des Verfassers.

345 Tehran \#150, 24.9.60, 788.00/9-2460, CF 60-63; „confused manipulation“ war eine frühe, noch etwas freundlichere Einschätzung von State Department und CIA. Vgl. State \#406, 10.8.60, deklassifiziert infolge eines FOIA-Antrags [NW 21263] des Verfassers. Selbstverständlich waren auch der zweifelhafte Volkstribun Mozaffar Bağā'i und die von ihm kontrollierte „Arbeiterpartei“ (hezb-e zahmatkašān) bei dieser Gelegenheit involviert. Asadollah "Alam erwartete „,more freedom in the coming elections" und einen Wahlsieg seiner Mardom. Die Botschaft berichtete über Versuche beider ,Gewächshaus"-Parteien, ihr Image in der iranischen Bevölkerung zu verbessern. So habe es bereits auf dem 1. Parteitag der Mardom im Oktober 1959 Versuche gegeben, die Partei in eine glaubwürdige Oppositionspartei zu verwandeln - offenbar versuchten ehemalige linke Kräfte, teilweise der Tudeh bzw. den Nationalisten nahestehend, Kontrolle über die Parteiorgane zu erlangen. Hier tauchte bereits die Bezeichnung ,Weiße Revolution“ zum ersten Mal auf. Vgl. Tehran \#788, 6.6.60, 788.00/6-660, CF 60-63; Jones Letter, 7.7.60, ,Correspondence with Ambassador', NEA, GTI: RIAD, 1958-63, Box 1; vgl. Tehran \#298, 4.8.60, FRUS 58-60, XII, S. 692f.; allerdings existierte innerhalb des etablierten Systems der Wahlfälschung eine Art Pluralismus, da verschiedene Machtzentren miteinander konkurrierten, was zu vielschichtigen Wahlabsprachen führte: ,The elections did produce parliaments representative of the major interests in each constituency but not,
} 
Statt der üblichen Resignation angesichts der Wahlfälschung regten sich nun aber erheblicher Widerstand und Kritik - Mohammad Rezā reagierte, aus Sicht der US-Botschaft überstürzt, im August mit der Annullierung der Wahlen und der Abberufung der Eg̉bāl-Regierung. Die Botschaft konstatierte das destabilisierende Moment, das die ,idea of free elections“ erzeugt habe und die Gefahren einer Abhaltung nicht-manipulierter Wahlen: ,[...] if an election were to be held in which anybody was free to participate, the probable result would be a Mosedaqist majority, an undetermined number of Tudeh deputies, and a group of provincial conservatives rich and influential enough to ensure their elections in their home districts." Der Schah könne einen echten Konstitutionalismus nicht gestatten und zudem wisse er nur zu gut, dass sein gegenwärtiges persönliches Regime , could not function as it has with such a Majlis.“" ${ }^{\text {"346 Das }}$ „Fiasko“ der abgebrochenen Wahlen verschärfte die innenpolitische Krise erheblich. CIADirektor Allen Dulles warnte den NSC, die Episode habe oppositionellen Strömungen in Iran neuen Auftrieb gegeben und ein Putschversuch sei nun wieder im Bereich des Möglichen. ${ }^{347}$

Die neue Regierung unter dem Premier Ğa ${ }^{`}$ far Šarif-Emāmi, etikettiert als Übergangsregierung, operierte bereits in einer Atmosphäre allgemeiner politischer Unsicherheit und vor dem Hintergrund einer zunehmend spürbareren ökonomischen Krise. Šarif-Emāmi, allgemein der Korruption und als Großmeister der Freimaurer in Iran zusätzlich der Servilität gegenüber Großbritannien verdächtigt, war eine durchaus zweifelhafte Wahl für das Krisenmanagement. ${ }^{348}$ Die neue Regierung verzettelte sich sogleich in Auseinandersetzungen

of course, representative of the population [...]."; CIA Research Study ,Elites and the Distribution of Power in Iran', Februar 1976, Asnād 7, S. 46; erst ab den 1960ern habe der Hof den Prozess landesweit kontrolliert. Zur Zweiten Nationalen Front vgl. CHEHABI, Liberation Movement, S. $143 \mathrm{f}$.

${ }^{346}$ Despatch \#150, vorherig. Anm.; Marcy Memo, 25.8.60, FRUS 58-60, XII, S. 696; die Zulassung scharfer Kritik in der Presse führten nicht wenige auf Baxtijārs politische Ambitionen zurück. Vgl. Tehran \#66, 9.8.60, 788.00/8960, CF 60-63; im aserbaidschanischen Mijāndoāb schoss die Polizei sogar auf einige Demonstranten - es gab mehrere Todesfälle. Die CIA hatte Zugriff auf ein Gesprächsprotokoll der entscheidenden Sitzung mit dem Schah und war umfassend über die inneren Entwicklungen informiert. Sogar die Schah-loyale US-Botschaft verwahrte sich gegen den apodiktischen Zweifel des Iran Desk Officer in GTI ,[...] that any Iranian sincerely aspire to a truly democratic system, nor do I think Iran would survive for long under Western style parliamentary democracy."; Schott Letter, 12.4.60, ,Official-Informal Letters from Tehran 1960“, NEA, GTI: RIAD, 1958-63, Box 1; zu den Umständen der abgebrochenen Wahlen zum 20. Mağles vgl. CotTAM, Iran and the United States, S. $122 \mathrm{ff}$; ANSARI, Modern Iran, S. 145f.; GASIOROWSKI, Client State, S. $177 \mathrm{ff}$.; zu den Beweggründen der tentativen Liberalisierung vgl. CHEHABI, Two Liberalizations.

${ }^{347}$ Die Einschätzung als „Fiasko“ seitens der US-Botschaft. Die auf Aufforderung des Schahs erfolgte Aufgabe der Mandate seitens der bereits gewählten Abgeordneten ermöglichte es, eine verfassungsrechtlich zweifelhafte formelle Annullierung zu umgehen. Siehe FRUS 58-60, XII, S. 696 n1, 698 n3; ed. note, ebd., S. 698f.; CIAInformationen zufolge plante der Schah eine effizienter umgesetzte Manipulation des nächsten Wahlgangs und womöglich eine inszenierte Wahlniederlage der „Regierungspartei“ sowie die Wahl einiger unabhängiger Kandidaten bzw. einiger Mitglieder der geduldeten neuen „Liberalen Partei“ Aminis. Allerdings sollte ,the kind of [...] free-for-all“" während des ersten Versuchs unterbunden werden. Vgl. Tehran \#317, 5.12.60, 788.00/12-560, CF 60-63; vgl. MiLANI, The Shah, S. 245f.; der Schah selbst bezichtigte nach der Revolution die Ölgesellschaften, die Unruhen in dieser Zeit angezettelt zu haben, um sich für seine Geschäfte mit Mattei zu rächen. Siehe PAHLAVI, Answer to History, S. 22.

${ }^{348}$ Vgl. GasiorowSKI, Client State, S. 179f.; zu Šarif-Emāmi vgl. BiLl, Eagle and Lion, S. 103; MilANI, The Shah, S. 247f.; ders., Eminent Persians: I, S. 305-10; vgl. LADJEVARDI, Xāterāt-e Šarif-Emāmi, S. 233-43; eine CIA-Studie von 1976 verwies ebenfalls auf britische Verbindungen Šarif-Emāmis. So habe er enge Beziehungen zu den MI6-nahen Rašidijān-Brüdern unterhalten. Vgl. CIA Research Study, Elites and the Distribution of Power in Iran“, Februar 1976, Asnād 7, S. 37; derselbe CIA-Bericht erwähnt zudem eine Intrige aus dem Jahr 1959, Ġolām Rezā, den Halbbruder des Schahs, offiziell zum Kronprinzen ernennen zu lassen. Sämtliche Verschwörer hätten der Grand Orient Masonic-Loge angehört, ,,an organization which the British apparently had used to promote their interests in Iran."; ebd., S. 67; COTTAM, Nationalism in Iran, S. 300f., nennt Šarif-Emāmi sogar 
mit der Militärführung über die notwendigen Kürzungen infolge des Stabilisierungsprogramms, während Šarif-Emāmis Schwager Ahmad Ārāmeš an der Spitze der Planorganisation die bisher geleistete Aufbauarbeit systematisch sabotierte. ${ }^{349}$ Alarmierend aus amerikanischer Sicht war zudem die Wiederaufnahme der sowjetisch-iranischen Verhandlungen über eine Garantie Teherans, keine Raketenstützpunkte auf dem eigenen Territorium zu genehmigen, sowie neuerliche Gerüchte, Mohammad Rezā zöge einen Austritt aus der CENTO ernsthaft in Erwägung. ${ }^{350}$

Das Endresultat nach acht Jahren amerikanischer Iranpolitik unter Präsident Eisenhower waren somit eine gefährliche Wirtschafts- und Zahlungsbilanzkrise, erhebliche Verschuldung, politische Instabilität und eine absehbare Krise im Zuge der für Anfang 1961 anberaumten Neuwahlen. ${ }^{351}$ Die überambitionierten Entwicklungsziele blieben unerreicht, die zentrale Planorganisation war am Ende der Dekade weitgehend dysfunktional. Die enormen, vom amerikanischen Steuerzahler subventionierten Aufrüstungsanstrengungen hatten eine übergroße, wenngleich kaum kampfbereite Armee geschaffen, die in erster Linie eine wachsende Belastung für die wirtschaftliche Entwicklung des Landes war. ${ }^{352}$ Schah Mohammad Rezā wiederum zeigte sich gegenüber amerikanischen Ratschlägen und Versuchen

einen „one-time Mosadeqist“ und seine Ernennung als Versuch, das politische Programm der nationalistischen Opposition zu kooptieren. Šarif-Emāmi sollte im Zuge der Revolution im August 1978 ein weiteres Mal an die Spitze der Regierung berufen werden.

${ }^{349}$ Vgl. Tehran \#280, 22.11.60, 788.00/11-2260, CF 60-63; zu Ārāmeš in der Planorganisation vgl. Manouchehr Goodarzi OHI, Juli 1983, OHIC, S. 42ff.; Ārāmeš starb 1973 in einem Feuergefecht mit der Polizei, die ihn der Terrorunterstützung verdächtigte. Vgl. Eintrag vom 26.2.61, in: LILIENTHAL, Journals: V, S. 168-71.

${ }^{350}$ Die CIA hatte im September über derartige Überlegungen informiert. Siehe CIA, Central Intelligence Bulletin, 10.9.60, CREST: \#RDP79*001-3; der sowjetische Botschafter kehrte nach Teheran zurück, der Propagandakrieg wurde vorerst eingestellt. Šarif-Emāmi wurde im Oktober $1960 \mathrm{zu}$ einer Goodwill-Tour nach Moskau eingeladen, die dann auch im Frühjahr 1961 stattfand, wenngleich vorerst ohne Resultate. Vgl. CIA, NSC Briefing, 14.9.60, CREST: \#RDP79*014-0; Tehran \#1035, 11.2.61, 033.8861/2-1161, CF 60-63; ed. note, FRUS 58-60, XII, S. 698f.; in der inzwischen fest etablierten Rollenverteilung forderten Botschafter Wailes und das Country Team vor Ort die Kompromittierung des ökonomischen Stabilisierungsprogrammes durch aufgestockte Budgethilfe und weitreichendes Entgegenkommen in militärischen Fragen, um einen Schwenk in Richtung Neutralität zu verhindern (,We are playing for big stakes in Iran, and I think the game is going to be rougher.“), u.a. Nike AjaxLuftabwehrraketen, Century Class-Jagdbomber und moderne Panzer. Ein US-Offizieller kritisierte die Forderungen scharf. Die Botschaft fordere a ,fairly significant increase in military assistance“, mache aber zugleich „,no attempt whatsoever at a military justification“. Die bisherige Politik der USA, der ,continued heavy emphasis on military assistance“, so der Autor, ,will inevitably align us with the Shah rather than with the aspirations of the Iranian people."; dennoch stimmte das DOD einer begrenzten Beschleunigung des Aufrüstungsprogramms ein weiteres Mal zu. Siehe Seymour Weiss Memo, 7.9.60, ,Defense Affairs Iran DEF 15 Bases', Lot 65 D 172, G/PM, Correspondence Concerning the Establishment and Defense of U.S. Military and Naval Bases Overseas, 1957-1963, Box 1; vgl. Tehran \#543, 1.9.60, 788.5 MSP/9-160; Tehran \#587, 8.9.60, 788.5 MSP/9-860; Jones Memo, 20.9.60, 788.5 MSP/9-2060, CF 60-63; Dabney Letter, 6.10.60, ,3-A/1 MDAP 1960`, NEA, GTI: RIAD, 1958-63, Box 2; Bowling Memo, 21.11.60; Handschriftliche Notiz, o.D., ,3-A/1 U.S. Military Assistance to Iran, 1960‘; Rockwell Memo, 10.9.60, ,16 USSR-Iran Relations 1960‘, ebd.

${ }^{351}$ Die CIA warnte Ende 1960, ein neuerliches Wahl-,,Fiasko“ „,...] would again throw Iranian politics into turmoil, and possibly lead to a crisis comparable to those of the Mossadez \{sic! $\}$ era."; CIA, NSC Briefing, 19.12.60, CREST: \#RDP79*019-0.

${ }_{352}$ Eine Studie der RAND Corporation vom April 1959 hatte eine Rückführung des Umfangs der iranischen Streitkräfte zugunsten einer Neuverlagerung der Mittel auf Aufstandsbekämpfung und Anti-Subversion sowie die Konzentration auf militärische Infrastruktur zur Unterstützung einer US-Militärintervention empfohlen. Vgl. Mouser Memo, 27.1.60, 788.5 MSP/1-2760, CF 60-63; John Bowling zeigte sich amüsiert - zwar wüsste jeder, dass die ,size of the Iranian Army should be halved“. Doch übersähen die RAND-Studien die Tatsache, dass Mohammad Rezā einen ,deep psychological need to command large, showy, and modern armed forces“ habe. Ein GTI-Offizieller stellte die These auf, es sei ,[...] inescapably true that we are causing ourselves some long-range political damage through the MAP program" infolge der wahrscheinlichen Haltung eines Nachfolgeregimes. Siehe Bowling Memo, 22.9.60; Handschriftliche Notiz, o.D., , Military Assistance - General, 1960‘, NEA, GTI: RIAD, 1958-63, Box 1; Wriggins Memo, 14.12.61; Miner Memo, 15.1.62, ,Office Memoranda, 1962‘, Box 4, ebd. 
der Einflussnahme zunehmend halsstarrig - gleichzeitig reagierte er panisch auf jedes Anzeichen eines Vertrauensverlustes in Washington, was sich in permanenten Beschwerden über kritische Berichte in amerikanischen Presseorganen und kruden Versuchen, das eigene Bild in der US-Öffentlichkeit positiv zu beeinflussen, ausdrückte. ${ }^{353}$

Die angestrebte politische Einbindung der oppositionellen Nationalisten war in keiner Form gelungen - in der zahlenmäßig gewachsenen urbanen Mittelschicht war die Unzufriedenheit mit den herrschenden Verhältnissen unübersehbar. Am augenfälligsten war der amerikanische Einfluss im Bereich der Sicherheitsorgane - die Schahherrschaft war zwar politisch diskreditiert, aber infolge der großzügigen 1290-d-Programme weitgehend immunisiert gegen mögliche Aufstände seitens der Bevölkerung. ${ }^{354}$ Iran sei ohnehin ,in many respects a police state, albeit a poorly run one“, argumentierte der Iran Desk Officer in GTI zugunsten direkter Unterstützung für die Polizeikräfte. ${ }^{355}$ Wenig Reflektion gab es auch auf amerikanischer Seite, was den eigenen Beitrag zur Schaffung und zum Ausbau des bald allgegenwärtigen SAWAKs anging. Berichte aus den Provinzen warnten vor der ausufernden Macht des SAWAK - der US-Konsul in Isfahan berichtete, ,[...] many Isfahanis have been stating that even in the worst days of Reza Shah they had seen nothing like the control and power which SAVAK now reportedly possesses“. ${ }^{356}$ Die Absicht hinter der Iranpolitik der Eisenhower-Administration der vergangenen Jahre, das pro-westliche Regime des Schahs zu stützen, aber zugleich eine

${ }^{353}$ Vgl. bereits die kritische Reaktion auf die Lippman-Kolumnen Ende 1959. Siehe oben S. 204f.; Sana Tehran \#030530Z JW 35, 3.9.60, 788.00(W)/8-460, CF 60-63; vgl. Dorman/FARHANG, U.S. Press and Iran, S. 72-6; Mohammad Rezā folgte dem Rat des State Departments und beauftragte eine PR-Agentur in New York damit, sein Image in den USA zu verbessern. Vgl. Jones Letter, 18.5.59, ,Correspondence with Ambassador, 1959‘, Lot 61 D 407, Suppl. Subject Files Relating to Iran, 1956-59, OiCIA/GTI, Box 20; Rivinus Jr. Memo, 6.4.60, ,OCB 091 Iran Folder 5', Records of the OCB, Box 3; zugleich verfasste der Schah mit Unterstützung eines amerikanischen Ghostwriters seine Memoiren, laut Sir Roger Stevens ein ,brilliant public relations effort“" mit Blick auf die US-Öffentlichkeit. Vgl. London Incoming \#G-181, 16.8.60, 788.11/8-1660, CF 60-63; das Werk erschien noch 1960 unter dem Titel Mission for My Country.

354 Der OCB hatte bereits im Sommer 1957 gewarnt, die nationalistische Opposition betrachte die Sicherheitsorgane allgemein, aber auch gerade Polizei und Gendarmerie, als ,political weapons designed to maintain the existing government and, therefore, in their minds the status quo" und daher müsse man bezüglich der Unterstützung der internen Sicherheitsorgane ,exercise caution in order to avoid becoming identified with the political aspects of police measures [...].“; OCB Draft, 12.6.57, ,OCB 091 Iran (File \#5) (4)“, WHO, NSCS, OCB Central File Ser., Box 44; gleichzeitig legte man nun aber den Schwerpunkt auf Ausbau und Professionalisierung der Polizeikräfte. Eine handschriftliche Notiz zu einer Sitzung des NSC Planning Board sprach explizit von „repressive type (anti-people) forces“; Handwritten Note, o.D. [vermutlich November 1958], ,[Notebook] NSC 1958-59“, JFDP, Gerard C. Smith Ser., Box 3, DDEL; vgl. 388th NSC Meeting, 3.12.58, FRUS 58-60, IV, S. 434443; der Kontext war die Politikrichtlinie in der neuen Basic National Security Policy NSC 5010/1 vom Mai 1958, in ausgesuchten Ländern an der Peripherie ,to emphasize police and constabulary type forces for internal security purposes in lieu of large indigenous military establishments."; NSC 5810/1, 5.5.58, FRUS 58-60, III, S. 104.

355 ,In those instances when Iranians have been exposed to the use of force they have quickly abandoned their desire to march in the streets. The more efficient the force the less willing have the citizenry been to indulge in mob action."; Mouser Memo, 28.7.58, 788.5 MSP/7-2858, CF 55-59; vgl. Bell Memo, 26.5.59, ,W/MSC-NEA File-12/58-1959', Lot 61 D 288, Office Files of John O. Bell, 1957-61, Box 1; etwa eine halbe Million US-Dollar wurden schließlich für Training und Ausrüstung von neun ,riot control companies“ bewilligt. Vgl. Country Team Minutes, 1.7.59, , Iran Country Team Meetings 1959“, Lot 61 D 407, Suppl. Subject Files Relating to Iran, 195659, OiCIA/GTI, Box 20; Rountree Memo, o.D., ,Office Memoranda 1958', Lot 60 D 533, Subject Files Relating to Iran, 1951-1958, OiCIA/GTI, Box 11.

${ }^{356}$ Isfahan \#14, 29.10.60, 788.00/10-2960, CF 60-63; der US-Konsul in Täbris sah eher ein ,public image nearer to that of an inefficient Gestapo than an FBI“; Tabriz \#51, 17.12.60, 788.00/12-1760, ebd.; selbst alte CIAWeggefährten wie 'Abbās Farzānegān beschwerten sich bei den Amerikanern über die Auswüchse des SAWAK. Der Vizedirektor des SAWAK Pākrawān selbst distanzierte sich von der kontraproduktiven Repression seiner Organisation unter den nationalen Minderheiten. Siehe MemCon, 17.8.60, 788.00/8-3060; Isfahan \#31, 23.3.60, 788.52/3-2360; Tabriz \#84, 1.6.60, 788.00/6-160, ebd. 
Identifikation der USA mit den repressiven Machterhaltungsstrategien des autoritären Herrschers zu vermeiden, war augenfällig gescheitert.

In Ansätzen zeigten sich Bestrebungen während des Ausklangs der republikanischen Administration, die eigene Politik gegenüber den Staaten der Peripherie im Allgemeinen und Iran im Besonderen zu revidieren. Ein offenbar experimentell intendiertes Papier aus dem Policy Planning Staff des State Departments vom Februar 1960 mit dem Titel „Iran: A Test Case in Policy Formation“ deutete ein Umdenken an. Durchaus ikonoklastisch erklärte die Studie die Aufrechterhaltung der Unabhängigkeit Irans gegenüber der UdSSR als zentrales amerikanisches Politikziel, wohingegen pro-westliche Orientierung, Bewahrung der Herrschaft Mohammad Rezās, Aufbau militärischer Kapazitäten, fortgesetzte CENTO-Mitgliedschaft usw. ,[...] all carry some degree of intrinsic advantage but are desirable primarily insofar as they promote the major objective." Keines dieser „secondary objectives“ sei somit „sakrosankt“. Im Grundsatz war diese Feststellung gleichbedeutend mit einer Verringerung der strategischen Ambition gegenüber Iran:

As long as Iran firmly resists Soviet domination, it is not extremely vital for the US whether the government is that of the Shah or of some other constellation; whether Iran votes favorably in the UN; whether social reform and economic progress proceed a little faster or a little slower, or Iranian forces are somewhat stronger or somewhat weaker; whether Iran is in or out of CENTO $[\ldots] .{ }^{357}$

Da eine sowjetische militärische Invasion extrem unwahrscheinlich sei und die Erfolge amerikanischer Wirtschafts- und Finanzhilfe in der Vergangenheit eher überschaubar, empfahl die Studie des Planungsstabes konkret, zumindest die Option eines neutralistischen Irans unter einer alternativen Führung in Erwägung zu ziehen. ${ }^{358}$

Zum Amtsantritt der neuen demokratisch geführten Kennedy-Administration befasste sich auch die CIA systematisch mit dem Phänomen der Klientel-Beziehungen mit Staaten der Peripherie und ging der Frage nach, ob die USA ,adequate returns for the money and efforts“ zur Aufrechterhaltung solcher Beziehungen erwirtschafte. Die Studie vom April 1961 kam zu einer grundsätzlich skeptischen Bewertung der Patron-Klienten-Beziehung: „The relationship often requires that the US give hostages to fortune in the form of explicit or implied commitments to regimes over which we have limited control.“ Darüber hinaus sei problematisch, dass die USA in historischer Pfadabhängigkeit mit den herrschenden konservativen Eliten Bindungen eingegangen sei, die mit amerikanischer Hilfe versuchten, die traditionellen Institutionen in

\footnotetext{
${ }^{357}$ Study, drafted by Barnes, 11.2.60, ,Iran 1958-1961', Lot 67 D 548, S/P Records: 1957-1961, Box 141, RG 59, NACP; die Studie kritisierte detailliert die aus ihrer Sicht mangelnde Prioritätensetzung gegenüber Iran in der in NSC 5821/1 festgelegten Politik. Zentral war die Behauptung der Studie, dass die Wirkung sowohl diplomatischer Einflussnahme wie auch materieller Hilfeleistungen in den Folgejahren abnehmen werde und daher ein Kurswechsel geboten sei.

${ }^{358}$ Vgl. ebd.; Voraussetzung für eine solche Politikwende war aber dem Autor zufolge eine nachhaltige globale Détente zwischen den Supermächten.
} 
ihren jeweiligen Ländern zu bewahren. Somit sei die amerikanische Politik quasi standardmäßig dem Status Quo und der Stabilität verpflichtet. Dies erzeuge wiederum die Gefahr, dass revolutionärer Wandel in einem Staat sich immer auch gegen den jeweiligen Patron richte und somit gesteigerte negative Konsequenzen aus amerikanischer strategischer Perspektive mit sich brächte.

Die CIA empfahl daher eine ergebnisoffene Einzelfallprüfung der bestehenden Klientelbeziehungen: ,[...] a weighing of the advantages and liabilities involved in the relationship may indicate that overall US interests would best be served by disengagement. “359 Spezifisch für Iran gestand die CIA die ,close identification with a basically unpopular and, in the long run, probably unviable regime" als amerikanisches Hauptproblem ein. Laut CIA verfügten die USA über „großen“ Einfluss, um die Situation innerhalb Irans zu beeinflussen und man sei wohl in der Lage, den Schah zu ernsthaften Reformen und sogar zur Reduktion seines aufgeblähten Militärapparates zu zwingen, wenngleich dies keine Garantie für das politische Überleben des Regimes wäre. Dieses Vorgehen sei notwendig, um das ,conventional concept of a pro-West Iran in close treaty relationship with the US“ zu bewahren - die CIAAnalysten kamen aber zu dem Schluss, dass die Chancen einer Durchsetzung dieses Konzepts in der geänderten Weltlage eher ,questionable“ seien. ${ }^{360}$

Das sich abzeichnende Umdenken innerhalb der amerikanischen strategischen Eliten Anfang der 1960er Jahre stellte die etablierten Strukturen der amerikanisch-iranischen Beziehungen seit 1946 bzw. 1953 im Grundsatz in Frage. Nun ging es im Kern nicht mehr allein um eine Debatte über das Für und Wider eines entschiedeneren reformistischen Interventionismus vonseiten des Patrons mit dem Ziel die langfristige Stabilität der bestehenden Ordnung in Iran zu sichern. Stattdessen gab es nun Stimmen, die den strategischen Einfluss der USA in der iranischen Innenpolitik für überflüssig, wenn nicht sogar gefährlich befanden. In Anbetracht der langjährigen Partnerschaft zwischen beiden Staaten und angesichts der bestehenden und konkreten US-Interessen an Iran waren Ideen eines vollständigen strategischen Rückzugs wohl niemals mehrheitsfähig und angesichts der tradierten Eindämmungsvorstellungen hinter der amerikanischen Grand Strategy ohnehin zu ikonoklastisch. Das Infragestellen etablierter Gewissheiten aber sollte den Befürwortern einer konsequenten Unterstützung derjenigen Kräfte in Iran, die auf Anpassung an die westliche Moderne und gesellschaftlichen, wirtschaftlichen und politischen Wandel setzten, neuen Auftrieb verleihen.

\footnotetext{
${ }^{359}$ CIA Study, ,US Relations with Clients States‘, 13.4.61, ,Central Intelligence Agency: General, 1961: JanuaryApril', NSF, D\&A, Box 271, JFKL; die Studie untersuchte systematisch die Klientelbeziehungen zu einer Reihe von Staten mit engen Bindungen zu den USA, war sich aber zugleich der unvermeidbaren Willkür einer solchen Auswahl bewusst. Nach einem ,,common-sense rule“ hatte man Iran, die Türkei, Griechenland, Pakistan, SaudiArabien, Israel, Südkorea, Südvietnam, Taiwan, Thailand und Spanien ausgewählt, denkbare lateinamerikanische Beispiel aber aufgrund der historisch weiter zurückgreifenden Pfadabhängigkeit der Beziehungen aber ausgespart. Siehe ebd., S. 3.

${ }^{360}$ Annex ,Country Case Studies`, ebd.
} 
Für die Beziehungen zwischen Mohammad Rezā, dem autoritären Herrscher in Iran, und dem wichtigsten externen Unterstützer seiner dominanten Stellung im politischen Systems des Landes, brachen schwierige Zeiten an. Amerikanischer , ,[...] withdrawal of support for the Shah would almost certainly end his rule, and he can hardly fail to appreciate this", gab sich die CIA überzeugt. ${ }^{361}$ Trotz der Infragestellung der bisherigen US-Politik in Iran durch den Präsidenten wie auch vonseiten der Analysten in State Department und CIA zog kaum jemand die logische Konsequenz mit Bezug auf Mohammad Rezā selbst und seine zentrale Stellung im Gesamtgefüge der amerikanischen Strategie. Die US-Strategie hatte, im Grunde mit den ersten Ansätzen während des Zweiten Weltkriegs beginnend, auf einen pro-westlichen und proamerikanischen Hof gesetzt - die Dominanz der Monarchie im politischen System Irans aufzugeben war gleichbedeutend mit der Inkaufnahme eines weitreichenden Einflussverlustes der USA. Die in den Quellen immer wieder zu findenden Klagen über das Fehlen glaubwürdiger alternativer Führungsfiguren sind daher zweifelhaft, war es doch die amerikanische Politik selbst, die den Aufstieg und die Etablierung echter Alternativen mit ihrer nahezu bedingungslosen Unterstützung für die Schah-Diktatur und deren Ausführungsorgane unterbunden hatte. Kritische Bemerkungen zu den Führungsqualitäten des Schahs finden sich durchaus, wenngleich häufig mit Qualifizierungen bezüglich seiner eigentlich wohlwollenden Absichten versehen.

Weitaus schärfere und zugleich treffendere Analysen finden sich in britischen Dokumenten. Der scheidende britische Botschafter Roger Stevens konstatierte nach mehreren Jahren in Teheran scharfsinnig, dass angesichts des fortschreitenden sozio-ökonomischen Wandels allein eine ,popular government“, getragen vom freien demokratischen Willens des Volkes und eben nicht gestützt durch Polizei und fremde Mächte, dauerhafte Stabilität für Iran verspreche. Das hauptsächliche Hindernis auf dem Weg zu einer solchen Stabilität, so Stevens im Sommer 1958, bliebe Mohammad Rezā selbst, dessen charakterliche Defizite letztlich jede notwendige Reform im Keim erstickten. Er sei „,...] incapable of formulating, let alone executing, a really constructive policy of any kind“ und zugleich „more concerned with his own survival and predominance than with the welfare of his people [...]“. So sehr auch immer die gegenwärtige Politik Irans kongruent zu britischen Interessen sei und das Schah-Regime „distinctly the lesser evil“" im Vergleich zu den sich anbietenden Alternativen, so wichtig sei auf der anderen Seite, dass , ,...] we should not deceive ourselves about the sort of man, or the sort of régime, with which we are dealing. “362

361 CIA Study, Anm. 358.

${ }^{362}$ Stevens, eine der prägenden Figuren der britischen Nahostpolitik dieser Zeit offerierte in seinem valedictory despatch auch eine ausführliche Einschätzung des monarchischen Charakters: ,[...] he is indecisive, listens to all comers, surrounds himself with bad advice, is incapable of a positive policy, concentrates far too much into his own hands. [...] his qualities, which include great charm, quickness, a sense of humour, an astounding memory, a good sense of public relations, remarkable gifts of exposition, an extra-ordinary knack of impressing foreign visitors and a flair of diplomacy and international affairs which he has handled with great skill [...]. His lack of 
self-confidence, and of any confidence in others, is utterly corrosive, and at the same time breeds a surface arrogance which many people who have much to do with him find repellent [...]. The defects in his character far out-weigh his virtues.“; trotz dieser Einsichten verteidigte Stevens die britische Politik der ,non-interference“ als weiterhin adäquat, zumal man ohnehin in erster Linie an „Iran's foreign alignments“ Interesse habe, empfahl aber zumindest moralische Unterstützung für die liberale Opposition. Stevens to FO, No. 120, 21.8.58, PREM 11/3397, TNA:PRO. 